\title{
Assessment of Coal Geology, Resources, and Reserves in the Southwestern Powder River Basin, Wyoming
}

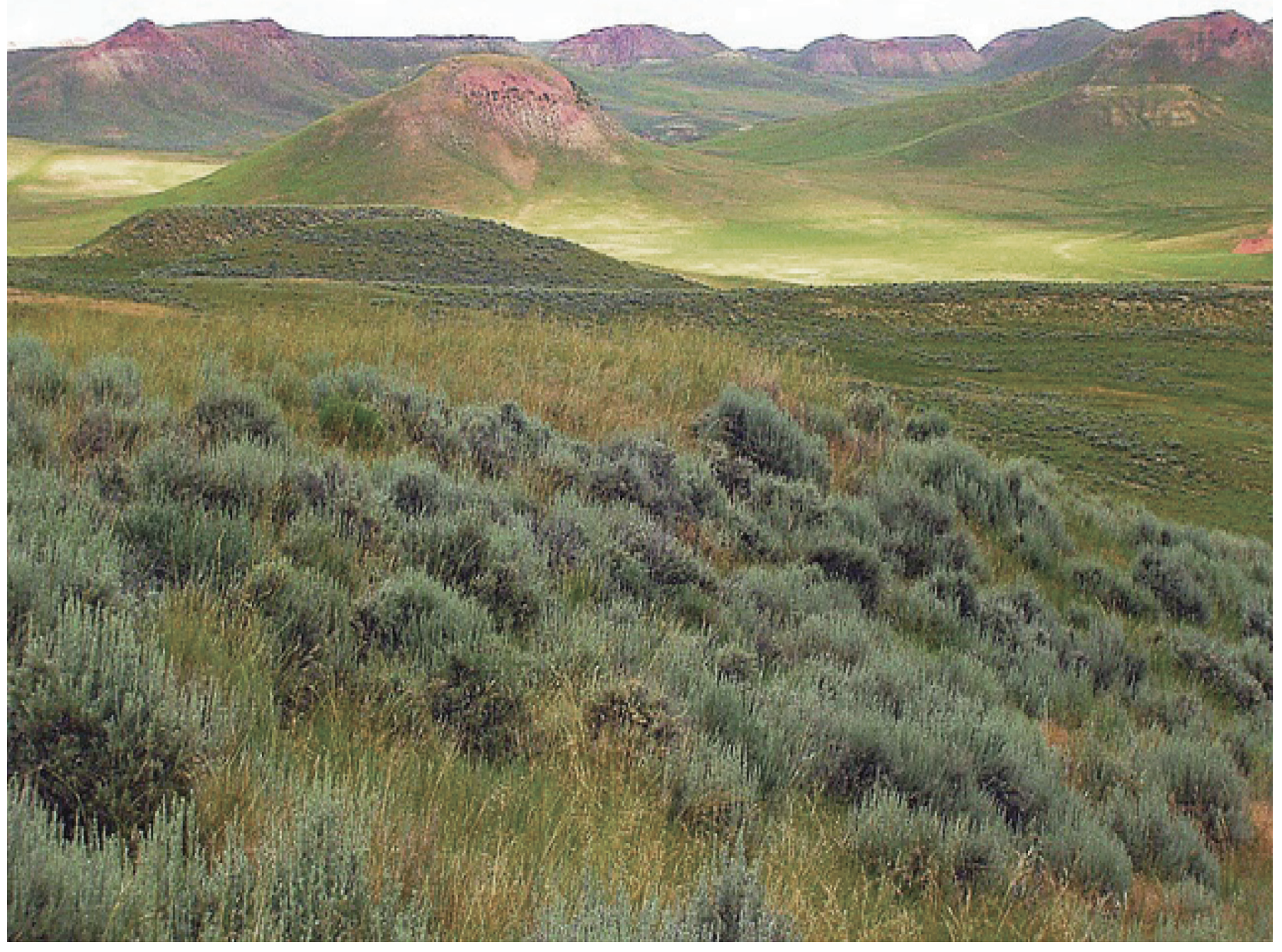

Open-File Report 2011-1134 
Cover. Photograph taken east of Buffalo, Wyoming, showing widespread clinker exposures. Photograph by Connie Modzelewski, Buffalo Field Office, Bureau of Land Management, Buffalo, Wyoming. 


\section{Assessment of Coal Geology, Resources, and Reserves in the Southwestern Powder River Basin, Wyoming}

By Lee M. Osmonson, David C. Scott, Jon E. Haacke, James A. Luppens, and

Paul E. Pierce

Open-File Report 2011-1134 


\title{
U.S. Department of the Interior \\ KEN SALAZAR, Secretary \\ U.S. Geological Survey \\ Marcia K. McNutt, Director
}

\author{
U.S. Geological Survey, Reston, Virginia: 2011
}

For more information on the USGS - the Federal source for science about the Earth, its natural and living resources, natural hazards, and the environment, visit http://www.usgs.gov or call 1-888-ASK-USGS.

For an overview of USGS information products, including maps, imagery, and publications, visit http://www.usgs.gov/pubprod

To order this and other USGS information products, visit http://store.usgs.gov

Any use of trade, product, or firm names is for descriptive purposes only and does not imply endorsement by the U.S. Government.

Although this report is in the public domain, permission must be secured from the individual copyright owners to reproduce any copyrighted materials contained within this report.

Suggested citation:

Osmonson, L.M., Scott, D.C., Haacke, J.E., Luppens, J.A., and Pierce, P.E., Assessment of coal geology, resources, and reserves in the Southwestern Powder River Basin, Wyoming: U.S. Geological Survey Open-File Report 2011-1134, $135 \mathrm{p}$. 


\section{Contents}

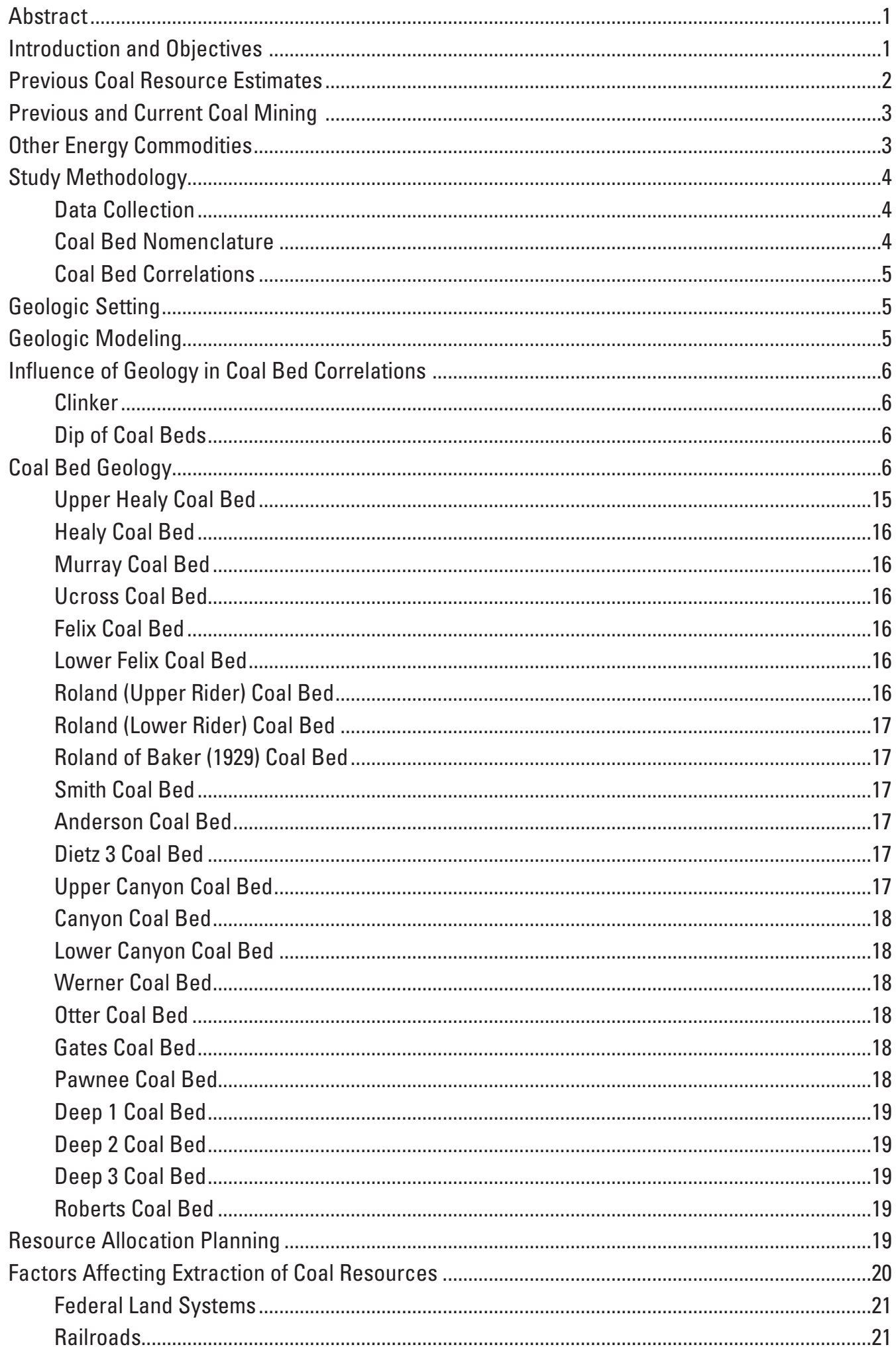




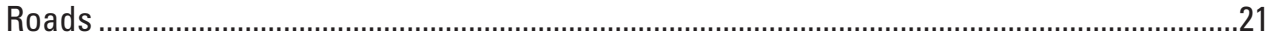

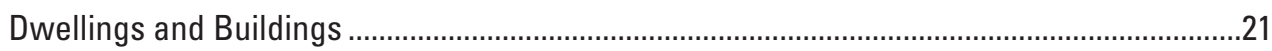

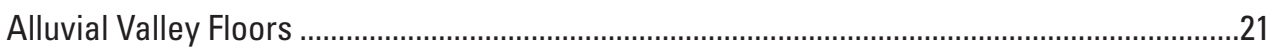

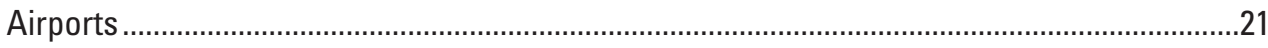

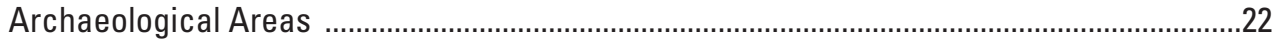

Coalbed Methane

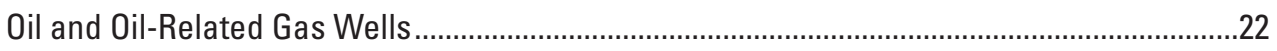

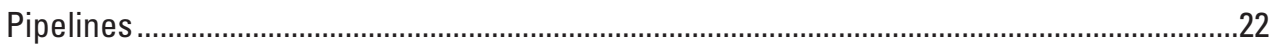

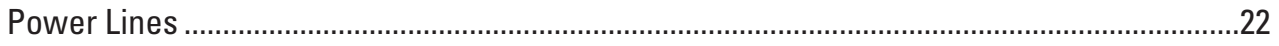

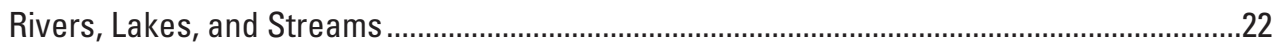

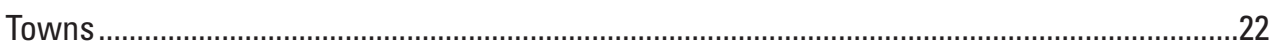

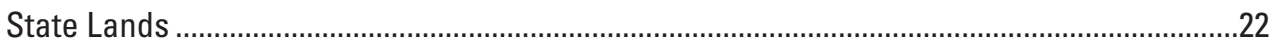

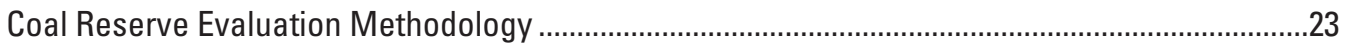

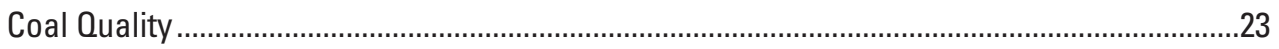

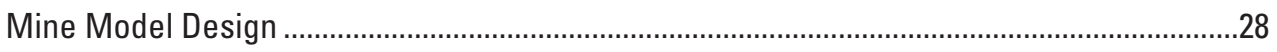

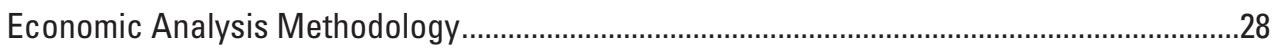

Underground Mine Model Assumptions ..............................................................................29

Resources Evaluation ....................................................................................................

Surface Coal Economic Analyses Results.............................................................................

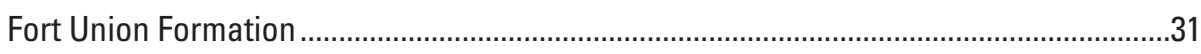

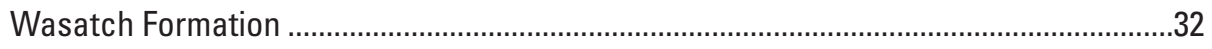

Underground Coal Economic Analysis Results ......................................................................

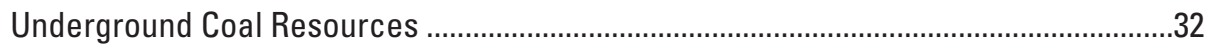

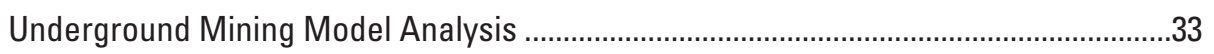

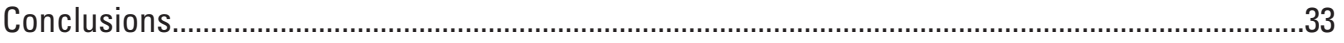

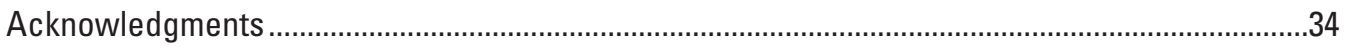

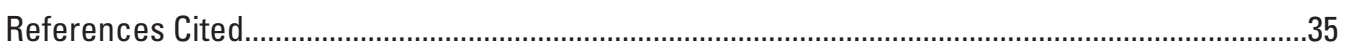

Glossary

\section{Figures}

1-4. Maps showing:

1. Location of Southwestern Powder River Basin assessment area, Northern Wyoming Powder River Basin assessment area, and Gillette coal field, Wyoming and Montana.

2. Coal fields in the Southwestern Powder River Basin assessment area...................41

3. Areas of coal resources in the Stevens Preference Right Lease Applications (PRLA's) in the Southwestern Powder River Basin assessment area.......................42

4. Drill hole locations in the Powder River Basin, Wyoming.......................................43

5. Flow chart showing generalized methodology used during coal resource and reserve evaluation.

6. Coal bed and coal zone names used in different publications in the Powder

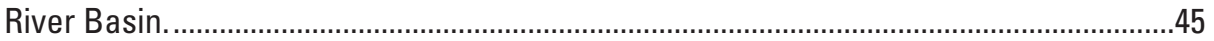

7. Generalized geology of the Powder River Basin, Wyoming and Montana.......................46 
8. Generalized stratigraphic column for the Powder River Basin, Wyoming and Montana .

9. Stratigraphy of coal beds in the southwestern Powder River Basin assessment area.

10. Map shwoing location of clinker in the southwestern Powder River

Basin assessment area

11. Stripping ratios for the Roland Baker and Smith coal beds in the southwestern Powder River Basin assessment area.

12-20. Cross sections:

12. West-east cross section $A-A^{\prime}$ through the northern part of the southwestern Wyoming Powder River Basin assessment area showing subsurface coal beds...

13. West-east cross section $B-B^{\prime}$ through the north-central part of the southwestern Wyoming Powder River Basin assessment area showing subsurface coal beds

14. West-east cross section $C-C^{\prime}$ through the central part of the southwestern Wyoming Powder River Basin assessment area showing subsurface coal beds

15. West-east cross section $D-D^{\prime}$ through the south-central part of the southwestern Wyoming Powder River Basin assessment area showing subsurface coal beds

16. West-east cross section $E-E^{\prime}$ through the southern part of the southwestern Wyoming Powder River Basin assessment area showing subsurface coal beds

17. South-north cross section $F-F^{\prime}$ through the central part of the southwestern Wyoming Powder River Basin assessment area showing subsurface coal beds

18. South-north cross section $G-G^{\prime}$ through the northern part of the southwestern Wyoming Powder River Basin assessment area showing subsurface coal beds

19. South-north cross section $H-H^{\prime}$ through the south-central part of the southwestern Wyoming Powder River Basin assessment area showing subsurface coal beds

20. South-north cross section $I-I^{\prime}$ through the south-eastern part of the southwestern Wyoming Powder River Basin assessment area showing subsurface coal beds

21-89. Maps showing:

21. Isopach map of the Upper Healy coal bed showing extent of resources greater than $2.5 \mathrm{ft}$ thick within the southwestern Powder River Basin assessment area.

22. Depth to the top of the Upper Healy coal bed within the southwestern Powder River Basin assessment area.

23. Coal resource reliability categories for the Upper Healy coal bed within the southwestern Powder River Basin assessment area.

24. Isopach map of the Healy coal bed showing extent of resources greater than $2.5 \mathrm{ft}$ thick within the southwestern Powder River Basin assessment area.

25. Depth to the top of the Healy coal bed within the southwestern Powder River Basin assessment area. 
26. Coal resource reliability categories for the Healy coal bed within the southwestern Powder River Basin assessment area.

27. Isopach map of the Murray coal bed showing extent of resources greater than $2.5 \mathrm{ft}$ thick within the southwestern Powder River Basin assessment area.

28. Depth to the top of the Murray coal bed within the southwestern Powder River Basin assessment area.

29. Coal resource reliability categories for the Murray coal bed within the southwestern Powder River Basin assessment area.

30. Isopach map of the Ucross coal bed showing extent of resources greater than $2.5 \mathrm{ft}$ thick within the southwestern Powder River Basin assessment area.

31. Depth to the top of the Ucross coal bed within the southwestern Powder River Basin assessment area.....

32. Coal resource reliability categories for the Ucross coal bed within the southwestern Powder River Basin assessment area

33. Isopach map of the Felix coal bed showing extent of resources greater than $2.5 \mathrm{ft}$ thick within the southwestern Powder River Basin assessment area.

34. Depth to the top of the Felix coal bed within the southwestern Powder River Basin assessment area

35. Coal resource reliability categories for the Felix coal bed within the southwestern Powder River Basin assessment area.

36. Isopach map of the Lower Felix coal bed showing extent of resources greater than $2.5 \mathrm{ft}$ thick within the southwestern Powder River Basin assessment area.

37. Depth to the top of the Lower Felix coal bed within the southwestern Powder River Basin assessment area.

38. Coal resource reliability categories for the Lower Felix coal bed within the southwestern Powder River Basin assessment area

39. Isopach map of the Roland (Upper Rider) coal bed showing extent of resources greater than $2.5 \mathrm{ft}$ thick within the southwestern Powder River Basin assessment area.

40. Depth to the top of the Roland (Upper Rider) coal bed within the southwestern Powder River Basin assessment area.

41. Coal resource reliability categories for the Roland (Upper Rider) coal bed within the southwestern Powder River Basin assessment area.

42. Isopach map of the Roland (Upper Rider) coal bed showing extent of resources greater than $2.5 \mathrm{ft}$ thick within the southwestern Powder River Basin assessment area.

43. Depth to the top of the Roland (Lower Rider) coal bed within the southwestern Powder River Basin assessment area.

44. Coal resource reliability categories for the Roland (Lower Rider) coal bed within the southwestern Powder River Basin assessment area.

45. Isopach map of the Roland Baker (1929) coal bed showing extent of resources greater than $2.5 \mathrm{ft}$ thick within the southwestern Powder River Basin assessment area.

46. Depth to the top of the Roland Baker (1929) coal bed within the southwestern Powder River Basin assessment area. 
47. Coal resource reliability categories for the Roland Baker (1929) coal bed within the southwestern Powder River Basin assessment area.............86

48. Isopach map of the Smith coal bed showing extent of resources greater than $2.5 \mathrm{ft}$ thick within the southwestern Powder River Basin assessment area.

49. Depth to the top of the Smith coal bed within the southwestern Powder River Basin assessment area.

50. Coal resource reliability categories for the Smith coal bed within the southwestern Powder River Basin assessment area.

51. Isopach map of the Anderson coal bed showing extent of resources greater than $2.5 \mathrm{ft}$ thick within the southwestern Powder River Basin assessment area.

52. Depth to the top of the Anderson coal bed within the southwestern Powder River Basin assessment area

53. Coal resource reliability categories for the Anderson

coal bed within the southwestern Powder River Basin assessment area

54. Isopach map of the Dietz 3 coal bed showing extent of resources greater than $2.5 \mathrm{ft}$ thick within the southwestern

Powder River Basin assessment area.

55. Depth to the top of the Dietz 3 coal bed within the southwestern Powder River Basin assessment area.

56. Coal resource reliability categories for the Dietz 3 coal bed within the southwestern Powder River Basin assessment area.

57. Isopach map of the Upper Canyon coal bed showing extent of resources greater than $2.5 \mathrm{ft}$ thick within the southwestern Powder River Basin assessment area.

58. Depth to the top of the Upper Canyon coal bed within the southwestern Powder River Basin assessment area

59. Coal resource reliability categories for the Upper Canyon coal bed within the southwestern Powder River Basin assessment area

60. Isopach map of the Canyon coal bed showing extent of resources greater than $2.5 \mathrm{ft}$ thick within the southwestern Powder River Basin assessment area

61. Depth to the top of the Canyon coal bed within the southwestern Powder River Basin assessment area.

62. Coal resource reliability categories for the Canyon coal bed within the southwestern Powder River Basin assessment area.

63. Isopach map of the Lower Canyon coal bed showing extent of resources greater than $2.5 \mathrm{ft}$ thick within the southwestern Powder River Basin assessment area.

64. Depth to the top of the Lower Canyon coal bed within the southwestern Powder River Basin assessment area.

65. Coal resource reliability categories for the Lower Canyon coal bed within the southwestern Powder River Basin assessment area

66. Isopach map of the Werner coal bed showing extent of resources greater than $2.5 \mathrm{ft}$ thick within the southwestern Powder River Basin assessment area.

67. Depth to the top of the Werner coal bed within the southwestern

Powder River Basin assessment area. 
68. Coal resource reliability categories for the Werner coal bed within the southwestern Powder River Basin assessment area.

69. Isopach map of the Otter coal bed showing extent of resources greater than $2.5 \mathrm{ft}$ thick within the southwestern Powder River Basin assessment area.

70. Depth to the top of the Otter coal bed within the southwestern Powder River Basin assessment area...

71. Coal resource reliability categories for the 0 tter coal bed within the southwestern Powder River Basin assessment area.

72. Isopach map of the Gates coal bed showing extent of resources greater than $2.5 \mathrm{ft}$ thick within the southwestern Powder River Basin assessment area.

73. Depth to the top of the Gates coal bed within the southwestern

Powder River Basin assessment area.

74. Coal resource reliability categories for the Gates coal bed within the southwestern Powder River Basin assessment area.

75. Isopach map of the Pawnee coal bed showing extent of resources greater than $2.5 \mathrm{ft}$ thick within the southwestern Powder River Basin assessment area.

76. Depth to the top of the Pawnee coal bed within the southwestern

Powder River Basin assessment area.

77. Coal resource reliability categories for the Pawnee coal bed within the southwestern Powder River Basin assessment area.

78. Isopach map of the Deep 1 coal bed showing extent of resources greater than $2.5 \mathrm{ft}$ thick within the southwestern Powder River Basin assessment area.

79. Depth to the top of the Deep 1 coal bed within the southwestern Powder River Basin assessment area

80. Coal resource reliability categories for the Deep 1 coal bed within the southwestern Powder River Basin assessment area.

81. Isopach map of the Deep 2 coal bed showing extent of resources greater than $2.5 \mathrm{ft}$ thick within the southwestern Powder River Basin assessment area.

82. Depth to the top of the Deep 2 coal bed within the southwestern Powder River Basin assessment area.

83. Coal resource reliability categories for the Deep 2 coal bed within the southwestern Powder River Basin assessment area.

84. Isopach map of the Deep 3 coal bed showing extent of resources greater than $2.5 \mathrm{ft}$ thick within the southwestern Powder River Basin assessment area.

85. Depth to the top of the Deep 3 coal bed within the southwestern Powder River Basin assessment area

86. Coal resource reliability categories for the Deep 3 coal bed within the southwestern Powder River Basin assessment area.

87. Isopach map of the Roberts coal bed showing extent of resources greater than $2.5 \mathrm{ft}$ thick within the southwestern Powder River Basin assessment area.

88. Depth to the top of the Roberts coal bed within the southwestern

Powder River Basin assessment area..... 
89. Coal resource reliability categories for the Roberts coal bed

within the southwestern Powder River Basin assessment area.

90. Illustration showing the effect of coal bed depth upon restricted resource due to mine pit highwall setback requirements .

91. Map of land use restrictions in the southwestern Powder River Basin assessment area

92. Map of coal ownership in the southwestern Powder River Basin assessment area...131

93. Estimated variability of as-received and moist, mineral-matter-free heating content values versus depth for the Wyodak-Anderson coal beds in the southwestern Powder River Basin assessment area

94. Diagram of typical longwall mining operation ..............................................................133

95. Map showing the areal extent for the Upper Healy and Healy coal beds .....................134

96. Map showing the areal extent for the Upper Healy and Healy coal beds ......................135

\section{Tables}

1. Coal resources reported in millions of short tons by reliability category for the southwestern Powder River Basin assessment area

2. Coal resources reported in millions of short tons, by coal ownership categories for the southwestern Powder River Basin assessment area

3. Coal resources reported in millions of short tons by overburden depth for the southwestern Powder River Basin assessment area...

4. Available as-received coal quality data from the Fort Union Formation in the southwestern Powder River Basin assessment area

5. Available as-received coal quality data from the Wasatch Formation in the Lake DeSmet area in the southwestern Powder River Basin assessment area.

6. Costs by underground mining case for the southwestern Powder River Basin assessment area based upon a 20 -year project life at an annual production rate of 6 million tons per year.

7. Costs of underground longwall mining for the southwestern Powder River Basin assessment area based on a 20 -year project life at an annual production rate of 6 million tons...

8. Contingency alternatives for estimating costs for longwall mining. . .30

9. Underground excavation dimensions in the longwall mining model for a 10 -foot mining height

10. Available resources for beds greater than or equal to 5.0 foot thick, overburden less than or equal to $500 \mathrm{feet}$, and $<10: 1$ strip ratio, reported in millions of short tons by stripping ratio for the southwestern Powder River Basin assessment area

11. Underground coal resources for the southwestern Powder River Basin assessment area 


\section{Abbreviations Used in This Report}

$\begin{array}{ll}\text { Btu/lb } & \text { British thermal unit per pound } \\ \mathrm{ft} & \text { feet } \\ \mathrm{mi} & \text { mile } \\ \mathrm{mi}^{2} & \text { square mile } \\ \mathrm{yr} & \text { year } \\ \mathrm{BST} & \text { billion short tons } \\ \mathrm{GIS} & \text { Geographic Information System } \\ \text { MST } & \text { million short tons } \\ \text { MST/yr } & \text { million short tons per year } \\ \text { NWPRB } & \text { Northern Wyoming Powder River Basin (assessment area) } \\ \text { PRB } & \text { Powder River Basin } \\ \text { PRLA } & \text { Preference Right Lease Application } \\ \text { PSO } & \text { lease area in Sheridan County; part of a land-exchange proposal } \\ \text { SWPRB } & \text { Southwestern Powder River Basin (assessment area) } \\ \text { ST } & \text { short ton } \\ \text { BLM } & \text { Bureau of Land Management } \\ \text { USGS } & \text { U.S. Geological Survey } \\ \text { UTM } & \text { Universal Transverse Mercator }\end{array}$

\section{Conversion Factors}

\begin{tabular}{lcl}
\hline \multicolumn{1}{c}{ Multiply } & By & \multicolumn{1}{c}{ To obtain } \\
\hline foot (ft) & Length & \\
mile (mi) & 0.3048 & meter $(\mathrm{m})$ \\
square mile $\left(\mathrm{mi}^{2}\right)$ & 1.609 & kilometer $(\mathrm{km})$ \\
square feet $\left(\mathrm{ft}^{2}\right)$ & 2.590 & square kilometer $\left(\mathrm{km}^{2}\right)$ \\
& 0.0929 & square meters $\left(\mathrm{m}^{2}\right)$ \\
\hline pounds $(\mathrm{lbs})$ & Density & \\
short tons $(2,000 \mathrm{lbs})$ & 0.4536 & kilograms $(\mathrm{kg})$ \\
millions of short tons $(\mathrm{MST})$ & 0.9072 & metric tons $(2,204.6 \mathrm{lbs})$ \\
billions of short tons (BST) & $1,000,000,000$ & short tons \\
trillions of short tons (TST) & $1,000,000,000,000$ & short tons \\
\hline & Area & short tons \\
\hline acre- $\mathrm{ft}$ & 1,770 & short tons of subbituminous coal \\
\hline
\end{tabular}




\title{
Assessment of Coal Geology, Resources, and Reserves in the Southwestern Powder River Basin, Wyoming
}

\author{
By Lee M. Osmonson, ${ }^{1}$ David C. Scott, Jon E. Haacke,, James A. Luppens, ${ }^{2}$ and Paul E. Pierce ${ }^{2}$
}

\section{Abstract}

The availability of abundant new borehole data from recent coal bed natural gas development was utilized by the U.S. Geological Survey for a comprehensive evaluation of coal resources and reserves in the southwestern part of the Powder River Basin in Wyoming. This report on the Southwestern Powder River Basin assessment area represents the third area within the basin to be assessed, the first being for coal resources and reserves in the Gillette coal field in 2008, and the second for coal resources and reserves in the northern Wyoming area of the basin in 2010 .

There are no active coal mines in the Southwestern Powder River Basin assessment area. The only significant production is attributed to the Dave Johnston Mine, in the extreme southern part of the area, which accounted for some 104 million short tons of coal from 1959 to 2000. Several small mines were developed in the 1950s near the Lake DeSmet area in the northwestern part of the area; however, less than 25,000 short tons of coal were produced.

Eight coal beds are present at depths between 1,000 and 2,000 ft in the Southwestern Powder River Basin assessment area. Portions of these coal beds might be recovered by underground mining methods in the future; however, the lack of sufficient drill data precluded an economic study to assess the potential of surface mineable coal resources. Consequently, none of the coal resources in the Southwestern Powder River Basin assessment area were designated as reserves or recoverable coal.

A total of 37 coal beds were identified during this assessment, 23 of which were modeled and evaluated to determine in-place coal resources. The total original coal resource in the Southwestern Powder River Basin assessment area for these 23 coal beds, with no restrictions applied was calculated to be 369 billion short tons. Available coal resources, which are part of the original resource that is accessible for potential mine development after subtracting all restrictions, are about 341 billion short tons (92.4 percent of the total original resource). Approximately 61 percent are at depths between 1,000

${ }^{1}$ U.S. Geological Survey, Denver, Colorado 80225

${ }^{2}$ U.S. Geological Survey, Reston, Virginia 20192 and 2,000 ft, with a modeled price of about $\$ 30$ per short ton. Therefore, the majority of coal resources in the Southwestern Powder River Basin assessment area are considered sub-economic

\section{Introduction and Objectives}

The U.S. Geological Survey (USGS) completed the first digital National Coal Resource Assessment of in-place coal resources in 2009 for selected coal beds in the United States (Pierce and Dennen, 2009). These estimates do not, by themselves, provide all the information needed for resource planning. Calculations of that portion of the in-place coal resources that are economically recoverable (coal reserves) are equally important (Luppens and others, 2008). (Note: Technical terms used in this report requiring additional explanation are italicized when used for the first time and their definitions are given in the Glossary section at the end of the report.)

Confusion concerning the use of the terms "coal resources" and "coal reserves" is relatively common. The two terms have been used interchangeably; however, there are significant differences. Coal resources include those in-place tonnage estimates determined by summing the volumes for identified and hypothetical coal resources and coal zones of a minimum thickness and within certain depth limits (commonly 0-2,000 ft deep). Coal reserves, a subset of the coal resources, are considered economically producible at the time of classification (Pierce and Dennen, 2009). Luppens and others (2009) discuss the terms "coal resources" and "coal reserves" and summarize how coal availability and recoverability studies (CARS) are used to determine estimates of economically recoverable coal resources.

Previous estimates (see references in "Previous Coal Resource Estimates" section) of coal reserves have typically used average mining percentages to obtain volume estimates of recoverable coal. Those estimates are general and do not take into consideration the amount of coal that cannot be mined because of environmental concerns, geological constraints, coal loss owing to mining and preparation technology, or economic constraints. More recently, published studies by the USGS have indicated that application of sitespecific restrictions to estimates of available coal resources 
significantly reduces the amount of coal that is considered recoverable (Ellis and others, 2002).

The current USGS coal assessment project is the Powder River Basin (PRB) located in northeastern Wyoming and southeastern Montana (fig. 1). The PRB was subdivided into four areas to keep the databases and modeled areas to a more manageable size and permit a more timely publication of assessment results. Luppens and others (2008) summarized the first area that contained coal resource and reserve estimates for the Gillette coal field (fig. 1). Scott and others (2010) summarized the second area that contained coal resource and reserve estimates for the Northern Wyoming Powder River Basin assessment area (NWPRB) (fig. 1).

This report summarizes the third area within the PRB and covers an area of approximately 4,750 square miles $\left(\mathrm{mi}^{2}\right)$ in the southwestern part of the PRB in portions of Johnson, Campbell, Converse, Natrona, and Niobrara Counties, Wyoming. The assessment area extends from the southern boundary of the NWPRB assessment area to the southern margin of the PRB, and eastward to the western boundary of the Gillette coal field (fig. 1). This part of the PRB is referred to in this report as the Southwestern Powder River Basin (SWPRB) assessment area. The fourth area to be assessed will be the Montana portion of the PRB.

Reliable coal resource and reserve estimates of these areas are essential for use in local, State, and Federal energy and land-use policy decisions for the foreseeable future. Additionally, this information can aid planners in determining the possible socio-economic effects on the region as coal resources are developed and eventually approach depletion (Luppens and others, 2008).

Coal availability and recoverability studies in the United States have previously relied on reinterpretation of existing data. Because of the recent development of coalbed methane in Wyoming and Montana, substantial volumes of new data from drill holes are now available. The interpretation of these new data provides a more accurate view of the coal resources and reserves in the PRB.

The primary objectives of this assessment were as follows:

1. Complete the third regional coal resource and reserve assessment in the PRB.

2. Improve geological assurance by updating the current stratigraphic database with information obtained from recently completed coalbed methane and oil and gas wells.

3. Develop a more comprehensive in-place coal resource computer model with the geological assurance to support regional reserve estimates.

4. Complete an economic mining evaluation that was customized to the environmental and technological restrictions in the SWPRB assessment area and derive a regional estimate of coal resources and reserves.
5. Complete the coal assessment for the Wyoming portion of the PRB.

\section{Previous Coal Resource Estimates}

Previous studies contain estimates of coal resources and reserves in the SWPRB assessment area as well as the other portions of the PRB. These studies have included different coal beds, coal zones, and boundaries, and have different purposes for which resources and reserves were calculated. Differences in criteria, such as variations in coal thickness and overburden categories have been used for those calculations (Luppens and others, 2008).

1. Mapel (1954) estimated 3 billion short tons (BST) of coal reserves in the Buffalo-Lake DeSmet area in beds more than $2.5 \mathrm{ft}$ thick and under less than $1,000 \mathrm{ft}$ of overburden (fig. 2). Several years later, Mapel (1959) estimated total coal reserves in the Buffalo-Lake DeSmet area in beds more than $2.5 \mathrm{ft}$ thick and under less than $1,000 \mathrm{ft}$ of overburden at about 6.4 BST.

2. Remaining strippable subbituminous coal reserves in several coal fields in the SWPRB assessment area were estimated by Smith (1972) and modified by Glass (1976). The Buffalo-Lake DeSmet area was estimated to contain about 1 BST; the Dry Cheyenne coal field was estimated to contain about 180 million short tons (MST); and the Sussex coal field was estimated to contain about 13 MST (fig. 2) (Glass, 1976).

3. In 1980, Glass estimated remaining strippable coal reserves at the Dave Johnston Mine in the Glenrock coal field at 91 MST (fig. 2) (Glass, 1980).

4. A report detailing the Final Environmental Assessment of the Belco-Bureau of Land Management (BLM) Coal Lease Exchange indicated that the Belco area, located just southeast of Buffalo, Wyoming contained between 137 and 185 MST of minable coal at a stripping ratio of 5:1 or less. The minable coal beds in the Belco area are the Healy and Ucross (fig. 2) (U.S. Bureau of Land Management, 1999).

5. In 1983, a report was submitted to the BLM for the lease acquisition of Preference Right Lease Applications (PRLA's) known as the Stevens North (W-12767, W-14390, W-14392), and the Stevens South (PRLA W-14355) (Western Fuels Association, Inc., 1983). These four PRLA's form one unit and are collectively known as the Stevens Project Area. In-place coal resources were estimated to be 625.3 MST for the entire area (figs. 2 and 3). 
As documented in these various estimates, resource and reserve estimates change as more data becomes available therefore, it is possible to refine previous resource and reserve estimates. These new estimates prove the usefulness of periodically recalculating coal resources and reserves. Because of the availability of new data, economic coal recoverability calculations in this report are significant refinements of previous coal resource and reserve studies in the SWPRB assessment area.

\section{Previous and Current Coal Mining}

In 1883, coal mining in the PRB began near the towns of Glenrock and Douglas, Wyoming (Ellis and others, 2002) (fig. 1). The subsequent development of railroad lines in 1886 and 1887 significantly influenced the growth of coal mining activity in the area (Ellis and others, 2002). The Barber, Buffalo, Dry Cheyenne, Glenrock, Lost Spring, Powder River, Pumpkin Buttes, and Sussex coal fields are all within the boundary of the SWPRB assessment area (fig. 2) (Glass, 1976). The first documented coal mining in the SWPRB assessment area began in the Buffalo coal field around the year 1900 (Mapel, 1959). Mapel (1959) defined a $640 \mathrm{mi}^{2}$ area and referred to it as the Buffalo-Lake DeSmet area (fig. 2). Between 1919 and 1950 several small mines were operated in the Lake DeSmet area, but with only minor production amounts. Approximately 19,000 short tons (ST) of coal were produced from the Kreselok Mine in section 2, T. 53 N., R. $82 \mathrm{~W}$. (fig. 2). A small strip mine was also operated in 1951 in section 3, T. 52 N., R. 82 W. (fig. 2), with recorded production of about 4,000 ST (Mapel, 1959). Total production from the Buffalo-Lake DeSmet area was less than 25,000 ST.

The only significant production in the SWPRB assessment area came from the Dave Johnston Mine in the Glenrock coal field (fig. 2). From 1958 to 2000, 104 MST of coal were produced from surface mining operations (Nick Jones, written commun., 2010). Reclamation began in 2000 after the mine was closed and was completed in 2005. A wind farm now occupies much of the reclaimed Dave Johnston Mine site. There is no current coal mining or production in the SWPRB assessment area.

\section{Other Energy Commodities}

Conventional oil and gas, along with coalbed methane are currently being developed in the SWPRB assessment area. Methane, however, is discussed as a separate gas resource because there are potential conflicts and concerns specific to its development that do not apply to conventional oil and gas development.

Powder River Basin oil and gas development began in 1887 with the discovery of an oil and gas field near Moorcroft, Wyoming (Ellis and others, 2002; fig. 1). According to the
Wyoming Oil and Gas Conservation Commission, 1,370 oil and gas wells were reported within the SWPRB assessment area as of January, 2010 (Wyoming Oil and Gas Conservation Commission, 2009) (fig. 4). Generally, there is little conflict between coal development and conventional oil and gas development in the SWPRB assessment area, as oil and gas reservoirs are primarily in stratigraphic units below minable coal beds.

Coalbed methane development in the PRB began in the early 1980s and as of January, 2010, 8,273 wells had been drilled in the SWPRB assessment area (fig. 4) (Wyoming Oil and Gas Conservation Commission, 2009). In 2003, an Environmental Impact Statement contained an analysis of potential impacts based on the assumption that by the year 2012 there will be about 51,000 producing private, State, and Federal coalbed methane wells and associated ancillary facilities in an almost 8 million acre area covering all or parts of Campbell, Converse, Johnson, and Sheridan Counties, Wyoming (U.S. Bureau of Land Management, 2003). Production life of coalbed methane wells is estimated to be about 10 to $12 \mathrm{yr}$, although production from multiple seams can extend the life of the well by an additional 10 to $30 \mathrm{yr}$ (De Bruin and others, 2004).

To produce coalbed methane, groundwater is pumped from the coal bed and can be discharged to holding ponds for consumption by livestock, discharged to existing drainage systems, released into the atmosphere through the use of misting towers, or re-injected into stratigraphic units. Concerns regarding possible contamination of existing surface water, the quality of water in holding ponds, the production of saline crust on the ground surface, the lowering of the water table, and possible contamination or depletion of groundwater in existing aquifers have all been examined in the PRB by Rice and others (2000).

Conflicts have arisen between coal mining and coalbed methane development. One conflict involves the ownership of coalbed methane whether it belongs to the owner of the oil and gas estate or the owner of the coal estate. The U.S. Supreme Court resolved this issue in 1999, when it ruled that coalbed methane is part of the oil and gas estate (Supreme Court of the United States, Amoco Production Co. vs. Southern Ute Tribe (98-830), 526, U.S. 865 (1999)). In addition, the court that specified the owner of the coalbed methane leases has the right to gain access and to develop its estate, and owners of the land surface should be adequately compensated for damage to their property resulting from coalbed methane extraction. The Bureau of Land Management has established Conflict Administration Zones to guide the development of coalbed methane leases in the path of near-term coal mining. In these zones, standard guidelines offer a process for settling conflicts and scheduling development of each resource under a Federal mineral estate. 


\section{Study Methodology}

The methodology for calculating coal resources and reserves in the SWPRB assessment area is described by the three phases shown on the flow chart in figure 5 . The first phase began with data collection and editing as a result of the acquisition of recently acquired geologic information. The second phase involved correlating coal beds, geologic modeling of those beds, resource allocation to determine land use, and technological restrictions within the SWPRB assessment area. The third phase involved completing a mining economics evaluation to determine coal reserves.

\section{Data Collection}

This report completes data collection and data interpretation for the Wyoming portion of the PRB. Over 44,750 wells have been drilled in the Wyoming portion of the PRB, of which, 18,370 drill holes were used to construct the database (fig. 4). This total represents oil and gas wells, coalbed methane wells, and coal exploration drill holes. Because of the huge volume of data, data not available for use, and data that were incomplete, only about 40 percent of the total was used. A total of 10,210 data points were used in the Gillette coal field report; 4,003 data points were used in the NWPRB assessment area report, and 4,157 data points were used in this report.

The database of Luppens and others (2008) was used as a basis for coal bed correlations along the eastern boundary of the SWPRB assessment area. Correlations from the NWPRB assessment area (Scott and others, 2010) were used as a basis for coal bed correlations in the northern part of the SWPRB assessment area.

As of September 2009, approximately 1,370 oil and gas wells and 8,273 coalbed methane wells had been drilled in the SWPRB assessment area. Data within a three-mile-wide buffer zone were added around the assessment area's boundary to extend stratigraphic correlations and to minimize the edge effect when the coal beds were modeled. The drill hole geophysical logs selected for data entry were downloaded as TIFF images from the Wyoming Oil and Gas Conservation Commission web site (2009). USGS personnel completed data entry for a total of 3,131 new geophysical well logs. In addition, the Wyoming State Geological Survey contributed 519 data points, and the USGS National Coal Resources Data System contributed 507 data points. The entire 4,157 data points were then entered into the StratiFact ${ }^{\circledR}$ database program for use in stratigraphic correlation (GRG Corporation, 1998).

Because the geophysical logs differ widely in quality and resolution, the interpretation of coal thickness is generally difficult. Gamma-ray logs were available for most of the wells and constituted the basis for most of the lithologic interpretation. Traditionally, oil and gas wells were logged primarily for detail in deep formations, and the upper (coal-bearing) intervals were either not logged or minimally gamma logged through the surface casing. Log data in older wells usually consisted only of spontaneous potential, resistivity and conductivity logs, making the identification of coal beds more difficult. The most reliable log suite consisted of natural gamma, gamma-gamma density, and resistivity traces. However, many coalbed methane wells were logged with gamma only, either in open hole or through steel drill pipe or casing. Additionally, many of those methane wells were logged with gamma ray only to the top of the target coal.

The primary focus of this report was to determine coal resources and reserves rather than to conduct a comprehensive geological study; therefore, detailed non-coal lithology types were not critical to the results of this evaluation. As a result, all lithology was coded as either coal or rock. Parting intercepts within coal beds and interburden between coal beds were also coded as rock. Intervals with no geophysical log such as the shallower part of an oil well or coalbed methane well that was not logged to the bottom, were entered as "No Log." However, if the methane-producing interval was available from production records, that interval was entered as coal.

\section{Coal Bed Nomenclature}

Correlation of individually named coal beds and coal zones across the entire PRB has been difficult, because the beds commonly split, merge, and pinch out (Flores and others, 1999). Also, in the past, distances between individual drill holes were often large (on the scale of 5-10 mi) increasing the uncertainty in correlating coal beds from one drill hole to the next. However, with the exploratory drilling and development of coalbed methane in the PRB, data from thousands of new drill holes are now available, and the distance between drill holes is thus reduced (fig. 4).

Many different names for individual coal beds and coal zones in the PRB have been used during the past $30 \mathrm{yr}$ (fig. 6). A report by Kent and others (1980), who described the northern part of the Gillette coal field that falls within the Spotted Horse coal field of Olive (1957), established a coal bed nomenclature system that has become the standard for much of the PRB in Wyoming. Kent and others (1980) retained certain existing coal bed nomenclature and revised other nomenclature by introducing new coal bed names. Molnia and Pierce (1992) also described coal bed stratigraphy in the central PRB in Wyoming and Montana; their nomenclature follows the usage of Culbertson and others (1979), Law and others (1979), Kent and others (1980), and Culbertson (1987). Flores and others (1999) defined a coal zone in the PRB known as the Wyodak-Anderson, which includes many named coal beds in the upper part of the Tongue River Member of the Fort Union Formation. The bed names used in this report and the equivalent names used in previous reports are found in figure 6 . For this assessment, resource calculations for individual coal beds composing the Wyodak-Anderson zone included the Smith, Anderson, Lower Anderson, Dietz 3, Upper Canyon, Canyon, Lower Canyon, and Werner. The stratigraphic framework for 
coal bed correlations in this report is based on the work of Luppens and others (2008), and Scott and others (2010).

\section{Coal Bed Correlations}

When all the drill hole data entry was completed, the graphical interface of the StratiFact (GRG Corporation, 1998) database was a critical tool for managing the interpretation of the large volume of information. With the StratiFact program, on-screen cross sections were selected, edited, and correlated. Both linear and circular cross sections were constructed to correlate coal beds across the SWPRB assessment area. Circular cross sections that verify closure were especially valuable when coal beds either split or thinned adjacent to sand channels. In this process, the beginning and ending drill holes of the cross section are the same, assuring that the coal beds do not cross each other.

Coal bed nomenclature used by Luppens and others (2008) in the Gillette coal field report and Scott and others (2010) in the Northern Wyoming Powder River Basin assessment report was used as a basis for correlating and naming coal beds between those two areas. Guidelines were established regarding which nomenclature would be used when two named beds merge into a single bed. The general correlation guidelines used for this assessment are as follows:

- Two named beds are considered to have merged into a single named bed when the intervening parting was less than $2.0 \mathrm{ft}$ thick. The following exceptions have been made for modeling purposes:

- In individual holes, coal beds split by partings as much as $5 \mathrm{ft}$ thick are considered to be merged if surrounding holes indicate the beds have merged into a single bed.

- In individual holes, coal beds with no partings or partings less than $2.0 \mathrm{ft}$ thick are considered to be split into two beds if surrounding holes show the coal has split into two beds.

- The upper bed's name will be used for the merged-bed name with one exception:

- A rider name becomes the main bed name; for example Roland (Baker, 1929) bed merged with Roland (Upper Rider) bed becomes Roland (Baker, 1929) bed.

\section{Geologic Setting}

The PRB covers about 22,000 $\mathrm{mi}^{2}$ in northeastern Wyoming and southeastern Montana (fig. 7). Near the west margin of the basin, the axis trends northwest-southeast and is distinctly asymmetrical with steep dips on the west side and gentle dips on the east (fig. 7). The Eocene Wasatch Formation covers about one-third of the PRB, mostly in Wyoming, and the underlying Paleocene Fort Union Formation is exposed along the basin margins in Wyoming and throughout most of the basin in Montana (figs. 7, 8). The Wasatch Formation conformably overlies the Fort Union Formation in the center of the basin and unconformably overlies it along the basin margins (fig. 8). The boundary between the two formations is generally placed above the Roland coal bed (fig. 8) (Flores and others, 1999). Rocks in the Fort Union Formation lie unconformably on the Upper Cretaceous Lance Formation (fig. 8). The Fort Union Formation in Wyoming is made up of three members, from upper to lower: the Tongue River Member, the Lebo Member, and the Tullock Member (fig. 8) (Ellis and others, 2002). The Fort Union Formation contains some of the thickest and most extensive deposits of subbituminous coal in the world (Molnia and Pierce, 1992). Most of this coal is in the Wyodak-Anderson coal zone in the Tongue River Member (Flores and others, 1999). The Wyodak-Anderson coal zone is equivalent to the following coal beds identified in this report (from youngest to oldest): Smith, Anderson, Lower Anderson, Dietz 1, Dietz 2, Dietz 3, Dietz 4, Canyon, Lower Canyon, Ferry, and Werner (figs. 6 and 9).

In general, Fort Union Formation coals range from subbituminous $\mathrm{C}$ to subbituminous $\mathrm{A}$ in apparent rank. The lower rank subbituminous $\mathrm{C}$ coal is located primarily in the shallower part of the basin (surface to 1,000-ft depth), the subbituminous $\mathrm{B}$ ranked coal is at an intermediate depth in the basin $(1,000-1,400-\mathrm{ft}$ depth), and subbituminous A ranked coal is in the deepest part of the basin (greater than 1,400-ft depth) (Stricker and others, 2007).

The SWPRB assessment area encompasses an area of about 5,585 $\mathrm{mi}^{2}$ in the western and southern parts of the PRB in Wyoming (fig. 7). The eastern boundary of the SWPRB assessment area is the western boundary of the Gillette coal field as defined by Luppens and others (2008). The northern boundary of this assessment area is part of the southern boundary of the NWPRB assessment area defined by Scott and others (2010). The western and southern boundaries of the SWPRB assessment area are defined by the contact of the Tertiary age rocks (fig. 7). Along the western edge of the PRB, strata dip between $20^{\circ}$ and $25^{\circ}$ to the east, whereas strata dip only about $2^{\circ}$ to $5^{\circ}$ to the west along the eastern edge of the basin (Flores and others, 1999). Surface coal mining has been more prevalent along the eastern margin of the basin because of the shallower dip of the coal beds.

\section{Geologic Modeling}

The first step of phase two of the assessment (fig. 5) was the creation of a digital coal bed model. Following coal bed correlations, preliminary coal isopach maps were created using the single bed mapping software Surfer ${ }^{\circledR}$ (Golden Software, 2002) to decide which coal beds would be included in the geological model. The basic criterion for inclusion of a coal bed was a minimum areal continuity of two or more townships. Twenty-three coal beds were selected and digital models 
for each bed were constructed. The integrated, multi-bed modeling program $\mathrm{PC} / \mathrm{Cores}^{\circledR}$ (Mentor Consultants, 2005) was used to produce the gridded geologic models. This modeling program was designed for coal or mineral evaluations and is more effective because of its capability to grid multiple beds at one time. The multiple gridding software allows for a considerable reduction in time when compared with other programs that grid only one parameter at a time.

The time required to produce all the required grids using a program that generates grids one parameter at a time can be large, considering that for each coal bed grids must be made for coal thickness, parting thickness, coal thickness (coal plus parting), and roof and floor structures. Then, grids must be calculated for the overburden and interburden for each seam, and then the roof grids for each coal bed must be individually subtracted from the surface grid or the floor grid for the next stratigraphically higher coal bed.

Most multiple-bed modeling programs are highly automated with a subroutine that uses individual coal bed structure grids to check for drill holes too shallow to penetrate a given bed. This feature produces more-accurate digital models without the need for extensive manual editing. The PC/Cores program code allows correlations to pass through the sections of the drill holes that were not geophysically logged to reduce the generation of false zero-thickness values.

The geologic models were gridded at a cell size of $150 \mathrm{~m}$ (about $500 \mathrm{ft}$ ). To verify coal bed correlations and coal bed areal distributions, preliminary roof and floor contour maps for each modeled bed were generated to check for "bull's eye" anomalies. A routine within the $\mathrm{PC} /$ Cores modeling program was used to identify suspect locations by comparing collar elevations to the digital elevation model of the Earth's surface. A number of location errors were resolved using this technique.

Once the editing was completed, the final geologic model was created and all the grids necessary for determination of the in-place coal resource volumes were generated. A copy of the in-place coal resources model was modified to generate the grids necessary for the coal reserve evaluation. The basic assumption used to qualify coal beds for potential coal reserve evaluation was to include coal beds that had a thickness of $2.5 \mathrm{ft}$ or greater. A final set of grids from both the coal resources and reserves models (thickness, parting thickness, and roof and floor structures) for each coal bed were converted in $\mathrm{PC} / \mathrm{Cores}$ to a generic ASCII grid format. These ASCII grids were then exported to the software program ArcView ${ }^{\circledR}$ Spatial Analyst (ESRI, 2001) to begin the last step in phase two of the assessment which was the modeling of the restrictions to mining.

\section{Influence of Geology in Coal Bed Correlations}

It is important to address the influence of geology as it relates to resources, reserves, and potential mining of the coal beds. The calculation of recoverable resources and reserves, for example, requires the identification of geological constraints that are likely to inhibit mining (Thomas, 2002). Such constraints in the SWPRB assessment area include clinker and dip of the coal beds.

\section{Clinker}

Coates and Heffern (1999) identified an area of about $110 \mathrm{mi}^{2}$ in the Lake DeSmet area that contains clinker (fig. 10) formed by the burning of the Lake DeSmet coal zone in the Wasatch Formation. The Lake DeSmet coal zone was originally correlated with the Healy coal bed (Mapel, 1959; Culbertson and Mapel, 1976). However, Obernyer (1978) indicated that the Lake DeSmet coal zone at Lake DeSmet represents the coalescing of five individual coal beds. From oldest to youngest, these are the Ucross, Murray, Cameron, Healy, and Walters (Glass and Jones, 1992). The depression of Lake DeSmet is thought to have been formed by the natural burning of this coal bed, causing subsidence of the land surface (Mapel, 1959).

Clinker is not considered a technical or land use restriction to mining in this report. However, clinker was included in the original resource calculation for each coal bed and later subtracted from each coal bed total to calculate the available coal resource.

\section{Dip of Coal Beds}

Another important factor in the evaluation of the resources and reserves in the SWPRB assessment area is the dip of the coal beds. As previously mentioned, the PRB is an asymmetrical basin with a northwest trending axis parallel to the western margin of the basin. Coal beds dip between $20^{\circ}$ and $25^{\circ}$ to the east along the western margin of the basin, whereas strata dip only about $2^{\circ}$ to $5^{\circ}$ to the west along the eastern margin of the basin. Stripping ratios for present surface mining along the eastern margin of the basin are between 1:1 and 3:1. Figure 11 shows the strip ratio for the Roland (Baker, 1929), Smith, Anderson, Dietz 3, Canyon, Lower Canyon, and Werner coal beds.

In summary, the calculation of recoverable resources and extractable reserves is dependent on the identification of geological constraints that are likely to inhibit mining. In the SWPRB assessment area, these geological constraints include the identification and location of clinker, and the dip of the coal beds.

\section{Coal Bed Geology}

During this evaluation thirty seven coal beds were identified in the SWPRB assessment area (fig. 9). Of that total, 23 beds were assessed for resource calculations (tables 1, 2, 3). 
Table 1. Coal resources reported in millions of short tons by reliability category for the Southwestern Powder River Basin assessment area. Resource includes coal plus partings. Reliability categories are based on distance from data point. Measured: < 1/4 mile; Indicated: 1/4-3/4 mile; Inferred: 3/4-3.0 miles; Hypothetical: > 3.0 miles. Totals may not sum exactly due to rounding.

\begin{tabular}{|c|c|c|c|c|c|c|c|}
\hline $\begin{array}{c}\text { Coal } \\
\text { bed } \\
\text { name }\end{array}$ & $\begin{array}{l}\text { Reliability } \\
\text { category }\end{array}$ & $\begin{array}{c}\text { Original } \\
\text { resources } \\
>2.5 \text { feet thick }\end{array}$ & $\begin{array}{c}\text { Previously } \\
\text { mined }\end{array}$ & $\begin{array}{l}\text { Land Use } \\
\text { restrictions }\end{array}$ & $\begin{array}{l}\text { Technical } \\
\text { restrictions }\end{array}$ & $\begin{array}{l}\text { Available } \\
\text { resources }\end{array}$ & $\begin{array}{l}\text { Percent of } \\
\text { total original } \\
\text { resource }\end{array}$ \\
\hline \multirow{5}{*}{$\begin{array}{l}\text { Healy } \\
\text { (Upper) }\end{array}$} & Measured & 461 & 0 & 34 & 13 & 414 & 8.5 \\
\hline & Indicated & 1,661 & 0 & 188 & 47 & 1,407 & 28.9 \\
\hline & Inferred & 2,581 & 0 & 624 & 299 & 1,658 & 34.1 \\
\hline & Hypothetical & 182 & 0 & 31 & 128 & 23 & 0.5 \\
\hline & Total & 4,865 & $\mathbf{0}$ & 877 & 487 & 3,502 & 72.0 \\
\hline \multirow[t]{5}{*}{ Healy } & Measured & 1,002 & 0 & 70 & 17 & 915 & 9.8 \\
\hline & Indicated & 3,331 & 0 & 393 & 55 & 2,883 & 30.8 \\
\hline & Inferred & 4,805 & 0 & 1,092 & 217 & 3,496 & 37.3 \\
\hline & Hypothetical & 234 & 0 & 81 & 60 & 93 & 1.0 \\
\hline & Total & 9,373 & $\mathbf{0}$ & 1,636 & 350 & 7,387 & 78.8 \\
\hline \multirow[t]{5}{*}{ Murray } & Measured & 242 & 0 & 2 & 177 & 63 & 4.2 \\
\hline & Indicated & 498 & 0 & 10 & 328 & 161 & 10.9 \\
\hline & Inferred & 706 & 0 & 18 & 503 & 184 & 12.4 \\
\hline & Hypothetical & 39 & 0 & 5 & 30 & 0 & 0.0 \\
\hline & Total & 1,481 & $\mathbf{0}$ & 35 & 1,038 & 408 & 27.5 \\
\hline \multirow[t]{5}{*}{ Ucross } & Measured & 242 & 0 & 22 & 28 & 192 & 3.9 \\
\hline & Indicated & 1,063 & 0 & 100 & 55 & 908 & 18.3 \\
\hline & Inferred & 3,231 & 0 & 661 & 224 & 2,346 & 47.4 \\
\hline & Hypothetical & 416 & 0 & 133 & 48 & 235 & 4.7 \\
\hline & Total & 4,952 & $\mathbf{0}$ & 916 & 354 & 3,381 & 74.3 \\
\hline \multirow[t]{5}{*}{ Felix } & Measured & 1,249 & 0 & 16 & 66 & 1,167 & 26.0 \\
\hline & Indicated & 2,208 & 0 & 26 & 134 & 2,048 & 45.5 \\
\hline & Inferred & 939 & 0 & 12 & 207 & 720 & 16.0 \\
\hline & Hypothetical & 102 & 0 & 0 & 76 & 26 & 0.6 \\
\hline & Total & 4,497 & $\mathbf{0}$ & 54 & 482 & 3,960 & 88.1 \\
\hline \multirow{5}{*}{$\begin{array}{l}\text { Felix } \\
\text { (Lower) }\end{array}$} & Measured & 1,288 & 0 & 21 & 435 & 832 & 7.8 \\
\hline & Indicated & 2,956 & 0 & 76 & 844 & 2,036 & 19.1 \\
\hline & Inferred & 5,632 & 0 & 568 & 476 & 4,589 & 43.0 \\
\hline & Hypothetical & 807 & 0 & 134 & 81 & 591 & 5.5 \\
\hline & Total & 10,683 & $\mathbf{0}$ & 798 & 1,837 & 8,048 & 75.3 \\
\hline \multirow{5}{*}{$\begin{array}{l}\text { Roland } \\
\text { (Upper } \\
\text { Rider) }\end{array}$} & Measured & 2,164 & 0 & 7 & 591 & 1,565 & 25.6 \\
\hline & Indicated & 3,052 & 0 & 7 & 962 & 2,083 & 34.0 \\
\hline & Inferred & 885 & 0 & 31 & 378 & 476 & 7.8 \\
\hline & Hypothetical & 18 & 0 & 0 & 18 & 0 & 0.0 \\
\hline & Total & 6,118 & $\mathbf{0}$ & 45 & 1,949 & 4,124 & 67.4 \\
\hline \multirow{5}{*}{$\begin{array}{l}\text { Roland } \\
\text { (Lower } \\
\text { Rider) }\end{array}$} & Measured & 680 & 0 & 0 & 205 & 475 & 21.8 \\
\hline & Indicated & 1,084 & 0 & 0 & 278 & 807 & 37.1 \\
\hline & Inferred & 412 & 0 & 0 & 151 & 261 & 12.0 \\
\hline & Hypothetical & 0 & 0 & 0 & 0 & 0 & 0.0 \\
\hline & Total & 2,177 & $\mathbf{0}$ & $\mathbf{0}$ & 634 & 1,543 & 70.9 \\
\hline
\end{tabular}


Table 1. Coal resources reported in millions of short tons by reliability category for the Southwestern Powder River Basin assessment area. Resource includes coal plus partings. Reliability categories are based on distance from data point. Measured: < 1/4 mile; Indicated: 1/4-3/4 mile; Inferred: 3/4-3.0 miles; Hypothetical: > 3.0 miles. Totals may not sum exactly due to rounding.-Continued

\begin{tabular}{|c|c|c|c|c|c|c|c|}
\hline $\begin{array}{c}\text { Coal } \\
\text { bed } \\
\text { name }\end{array}$ & $\begin{array}{l}\text { Reliability } \\
\text { category }\end{array}$ & $\begin{array}{c}\text { Original } \\
\text { resources } \\
>\mathbf{2 . 5} \text { feet thick } \\
\end{array}$ & $\begin{array}{c}\text { Previously } \\
\text { mined }\end{array}$ & $\begin{array}{l}\text { Land Use } \\
\text { restrictions }\end{array}$ & $\begin{array}{l}\text { Technical } \\
\text { restrictions }\end{array}$ & $\begin{array}{l}\text { Available } \\
\text { resources }\end{array}$ & $\begin{array}{c}\text { Percent of } \\
\text { total original } \\
\text { resource }\end{array}$ \\
\hline \multirow{5}{*}{$\begin{array}{l}\text { Roland of } \\
\text { Baker } \\
(1929)\end{array}$} & Measured & 2,122 & 0 & 62 & 416 & 1,644 & 16.9 \\
\hline & Indicated & 4,053 & 0 & 50 & 606 & 3,397 & 34.9 \\
\hline & Inferred & 3,453 & 0 & 54 & 456 & 2,944 & 30.3 \\
\hline & Hypothetical & 98 & 0 & 1 & 92 & 5 & 0.1 \\
\hline & Total & 9,727 & $\mathbf{0}$ & 167 & 1,570 & 7,990 & 82.1 \\
\hline \multirow[t]{5}{*}{ Smith } & Measured & 27,262 & 12 & 7 & 86 & 27,157 & 30.9 \\
\hline & Indicated & 47,532 & 0 & 42 & 299 & 47,190 & 53.8 \\
\hline & Inferred & 12,676 & 0 & 309 & 618 & 11,749 & 13.4 \\
\hline & Hypothetical & 275 & 0 & 131 & 70 & 75 & 0.1 \\
\hline & Total & 87,745 & 12 & 490 & 1,073 & 86,171 & 99.2 \\
\hline \multirow[t]{5}{*}{ Anderson } & Measured & 2,596 & 0 & 26 & 129 & 2,440 & 26.4 \\
\hline & Indicated & 4,757 & 0 & 15 & 350 & 4,391 & 47.5 \\
\hline & Inferred & 1,883 & 0 & 0 & 304 & 1,579 & 17.1 \\
\hline & Hypothetical & 17 & 0 & 0 & 0 & 17 & 0.2 \\
\hline & Total & 9,253 & $\mathbf{0}$ & 41 & 784 & 8,428 & 91.1 \\
\hline \multirow[t]{5}{*}{ Dietz 3} & Measured & 3,643 & 0 & 24 & 52 & 3,567 & 29.7 \\
\hline & Indicated & 5,456 & 0 & 15 & 157 & 5,284 & 44.1 \\
\hline & Inferred & 2,885 & 0 & 0 & 342 & 2,543 & 21.2 \\
\hline & Hypothetical & 7 & 0 & 0 & 7 & 0 & 0.0 \\
\hline & Total & 11,992 & $\mathbf{0}$ & 39 & 558 & 11,395 & 95.0 \\
\hline \multirow{5}{*}{$\begin{array}{l}\text { Canyon } \\
\text { (Upper) }\end{array}$} & Measured & 807 & 0 & 2 & 26 & 788 & 15.0 \\
\hline & Indicated & 1,834 & 0 & 13 & 71 & 1,750 & 33.7 \\
\hline & Inferred & 2,420 & 0 & 80 & 317 & 2,024 & 39.0 \\
\hline & Hypothetical & 131 & 0 & 3 & 84 & 44 & 0.8 \\
\hline & Total & 5,192 & $\mathbf{0}$ & 98 & 498 & 4,596 & 88.5 \\
\hline \multirow[t]{5}{*}{ Canyon } & Measured & 19,178 & 0 & 6 & 42 & 19,130 & 29.0 \\
\hline & Indicated & 30,919 & 0 & 31 & 131 & 30,757 & 46.6 \\
\hline & Inferred & 14,748 & 0 & 278 & 402 & 14,067 & 21.3 \\
\hline & Hypothetical & 1,105 & 0 & 104 & 64 & 937 & 1.4 \\
\hline & Total & 65,951 & $\mathbf{0}$ & 420 & 639 & 64,891 & 98.4 \\
\hline \multirow{5}{*}{$\begin{array}{l}\text { Canyon } \\
\text { (Lower) }\end{array}$} & Measured & 7,123 & 0 & 20 & 71 & 7,032 & 23.9 \\
\hline & Indicated & 14,884 & 0 & 29 & 238 & 14,617 & 49.8 \\
\hline & Inferred & 7,052 & 0 & 84 & 421 & 6,547 & 22.3 \\
\hline & Hypothetical & 310 & 0 & 2 & 162 & 145 & 0.5 \\
\hline & Total & 29,368 & $\mathbf{0}$ & 136 & 892 & 28,341 & 96.5 \\
\hline \multirow[t]{5}{*}{ Werner } & Measured & 1,564 & 0 & 7 & 126 & 1,432 & 13.0 \\
\hline & Indicated & 4,295 & 0 & 37 & 427 & 3,830 & 34.8 \\
\hline & Inferred & 4,685 & 0 & 253 & 475 & 3,958 & 35.9 \\
\hline & Hypothetical & 473 & 0 & 70 & 52 & 351 & 3.2 \\
\hline & Total & 11,017 & $\mathbf{0}$ & 367 & 1,080 & $\mathbf{9 , 5 7 1}$ & 86.9 \\
\hline
\end{tabular}


Table 1. Coal resources reported in millions of short tons by reliability category for the Southwestern Powder River Basin assessment area. Resource includes coal plus partings. Reliability categories are based on distance from data point. Measured: < 1/4 mile; Indicated: 1/4-3/4 mile; Inferred: 3/4-3.0 miles; Hypothetical: > 3.0 miles. Totals may not sum exactly due to rounding.-Continued

\begin{tabular}{|c|c|c|c|c|c|c|c|}
\hline $\begin{array}{c}\text { Coal } \\
\text { bed } \\
\text { name }\end{array}$ & $\begin{array}{l}\text { Reliability } \\
\text { category }\end{array}$ & $\begin{array}{c}\text { Original } \\
\text { resources } \\
>2.5 \text { feet thick }\end{array}$ & $\begin{array}{c}\text { Previously } \\
\text { mined }\end{array}$ & $\begin{array}{l}\text { Land Use } \\
\text { restrictions }\end{array}$ & $\begin{array}{l}\text { Technical } \\
\text { restrictions }\end{array}$ & $\begin{array}{l}\text { Available } \\
\text { resources }\end{array}$ & $\begin{array}{l}\text { Percent of } \\
\text { total original } \\
\text { resource }\end{array}$ \\
\hline \multirow[t]{5}{*}{ Otter } & Measured & 6,314 & 0 & 43 & 69 & 6,202 & 21.8 \\
\hline & Indicated & 14,452 & 0 & 36 & 211 & 14,205 & 49.8 \\
\hline & Inferred & 7,686 & 0 & 4 & 499 & 7,183 & 25.2 \\
\hline & Hypothetical & 62 & 0 & 0 & 62 & 0 & 0.0 \\
\hline & Total & 28,514 & $\mathbf{0}$ & 83 & 841 & 27,591 & 96.8 \\
\hline \multirow[t]{5}{*}{ Gates } & Measured & 2,350 & 0 & 6 & 92 & 2,252 & 10.8 \\
\hline & Indicated & 8,388 & 0 & 18 & 294 & 8,075 & 38.6 \\
\hline & Inferred & 9,766 & 0 & 128 & 489 & 9,148 & 43.7 \\
\hline & Hypothetical & 426 & 0 & 33 & 52 & 341 & 1.6 \\
\hline & Total & 20,929 & $\mathbf{0}$ & 185 & 927 & 19,817 & 94.7 \\
\hline \multirow[t]{5}{*}{ Pawnee } & Measured & 219 & 0 & 0 & 47 & 172 & 5.6 \\
\hline & Indicated & 845 & 0 & 0 & 179 & 665 & 21.8 \\
\hline & Inferred & 1,790 & 0 & 0 & 393 & 1,398 & 45.8 \\
\hline & Hypothetical & 194 & 0 & 14 & 65 & 115 & 3.8 \\
\hline & Total & 3,049 & $\mathbf{0}$ & 14 & 685 & 2,351 & 77.1 \\
\hline \multirow[t]{5}{*}{ Deep 1} & Measured & 1,323 & 0 & 2 & 106 & 1,215 & 12.8 \\
\hline & Indicated & 4,624 & 0 & 2 & 347 & 4,275 & 44.9 \\
\hline & Inferred & 3,574 & 0 & 0 & 373 & 3,201 & 33.6 \\
\hline & Hypothetical & 0 & 0 & 0 & 0 & 0 & 0.0 \\
\hline & Total & $\mathbf{9 , 5 2 1}$ & $\mathbf{0}$ & 4 & 826 & 8,691 & 91.3 \\
\hline \multirow[t]{5}{*}{ Deep 2} & Measured & 770 & 0 & 0 & 140 & 629 & 8.3 \\
\hline & Indicated & 2,664 & 0 & 2 & 528 & 2,133 & 28.2 \\
\hline & Inferred & 3,827 & 0 & 28 & 872 & 2,927 & 38.7 \\
\hline & Hypothetical & 297 & 0 & 0 & 47 & 250 & 3.3 \\
\hline & Total & 7,557 & $\mathbf{0}$ & 30 & 1,587 & 5,940 & 78.6 \\
\hline \multirow[t]{5}{*}{ Deep 3} & Measured & 1,397 & 0 & 1 & 100 & 1,296 & 9.3 \\
\hline & Indicated & 5,462 & 0 & 2 & 414 & 5,055 & 36.4 \\
\hline & Inferred & 6,915 & 0 & 2 & 921 & 5,992 & 43.1 \\
\hline & Hypothetical & 119 & 0 & 0 & 51 & 69 & 0.5 \\
\hline & Total & 13,903 & $\mathbf{0}$ & 6 & 1,486 & 12,412 & 89.3 \\
\hline \multirow[t]{5}{*}{ Roberts } & Measured & 836 & 0 & 1 & 62 & 772 & 6.9 \\
\hline & Indicated & 3,578 & 0 & 3 & 245 & 3,330 & 29.7 \\
\hline & Inferred & 6,683 & 0 & 11 & 654 & 6,018 & 53.6 \\
\hline & Hypothetical & 131 & 0 & 0 & 61 & 70 & 0.6 \\
\hline & Total & 11,229 & $\mathbf{0}$ & 16 & 1,022 & 10,191 & 90.8 \\
\hline \multirow[t]{5}{*}{ Total beds } & Measured & 84,832 & 12 & 379 & 3,099 & 81,342 & 22.0 \\
\hline & Indicated & 169,588 & 0 & 1,098 & 7,201 & 161,288 & 43.7 \\
\hline & Inferred & 109,235 & 0 & 4,238 & 9,988 & 95,009 & 25.7 \\
\hline & Hypothetical & 5,440 & 0 & 742 & 1,310 & 3,389 & 0.9 \\
\hline & Total & 369,095 & 12 & 6,458 & $\mathbf{2 1 , 5 9 7}$ & 341,029 & 92.4 \\
\hline
\end{tabular}


Table 2. Coal resources reported in millions of short tons, by coal ownership categories for the Southwestern Powder River Basin assessment area. Resource includes coal plus partings. Totals may not sum exactly due to rounding.

\begin{tabular}{|c|c|c|c|c|c|c|c|}
\hline $\begin{array}{c}\text { Coal } \\
\text { bed } \\
\text { name }\end{array}$ & $\begin{array}{c}\text { Coal } \\
\text { ownership }\end{array}$ & $\begin{array}{c}\text { Original } \\
\text { resources } \\
>2.5 \text { feet thick } \\
\end{array}$ & $\begin{array}{c}\text { Previously } \\
\text { mined }\end{array}$ & $\begin{array}{l}\text { Land Use } \\
\text { restrictions }\end{array}$ & $\begin{array}{c}\text { Technical } \\
\text { restrictions }\end{array}$ & $\begin{array}{l}\text { Available } \\
\text { resources }\end{array}$ & $\begin{array}{c}\text { Percent of } \\
\text { total original } \\
\text { resource }\end{array}$ \\
\hline \multirow{2}{*}{ Healy (Upper) } & State & 499 & 0 & 76 & 63 & 359 & 7.4 \\
\hline & Private & 1,228 & 0 & 532 & 160 & 536 & 11.0 \\
\hline \multirow{3}{*}{ Healy } & State & 1,186 & 0 & 175 & 64 & 847 & 9.0 \\
\hline & Private & 2,679 & 0 & 971 & 120 & 1,589 & 17.0 \\
\hline & Total & 9,373 & $\mathbf{0}$ & 1,636 & 350 & 7,387 & 78.8 \\
\hline Murray & Federal & 977 & 0 & 15 & 695 & 277 & 18.7 \\
\hline \multirow[t]{4}{*}{ Ucross } & Federal & 2,227 & 0 & 205 & 218 & 1,803 & 36.4 \\
\hline & State & 650 & 0 & 75 & 53 & 522 & 10.5 \\
\hline & Private & 2,075 & 0 & 636 & 83 & 1,356 & 27.4 \\
\hline & Total & 4,952 & $\mathbf{0}$ & 916 & 354 & 3,681 & 74.3 \\
\hline \multirow[t]{4}{*}{ Felix } & Federal & 4,013 & 0 & 54 & 342 & 3,617 & 80.4 \\
\hline & State & 251 & 0 & 0 & 31 & 220 & 4.9 \\
\hline & Private & 233 & 0 & 1 & 109 & 123 & 2.7 \\
\hline & Total & 4,497 & $\mathbf{0}$ & 54 & 482 & 3,960 & 81.1 \\
\hline Felix (Lower) & Federal & 7,206 & 0 & 147 & 1,548 & 5,511 & 51.6 \\
\hline $\begin{array}{l}\text { Roland } \\
\text { (Upper Rider) }\end{array}$ & Total & 6,119 & $\mathbf{0}$ & 45 & 1,949 & 4,124 & 67.4 \\
\hline \multirow{4}{*}{$\begin{array}{l}\text { Roland } \\
\text { (Lower Rider) }\end{array}$} & Federal & 1,915 & 0 & 0 & 565 & 1,350 & 62.0 \\
\hline & State & 185 & 0 & 0 & 40 & 145 & 6.7 \\
\hline & Private & 77 & 0 & 0 & 29 & 47 & 2.2 \\
\hline & Total & 2,177 & $\mathbf{0}$ & $\mathbf{0}$ & 634 & 1,543 & 70.9 \\
\hline \multirow{4}{*}{$\begin{array}{l}\text { Roland of } \\
\text { Baker (1929) }\end{array}$} & Federal & 8,174 & 0 & 110 & 1,208 & 6,856 & 70.5 \\
\hline & State & 694 & 0 & 5 & 114 & 574 & 5.9 \\
\hline & Private & 859 & 0 & 52 & 248 & 559 & 5.7 \\
\hline & Total & 9,727 & $\mathbf{0}$ & 167 & 1,570 & 7,990 & 82.1 \\
\hline \multirow[t]{4}{*}{ Smith } & Federal & 76,994 & 12 & 103 & 774 & 76,105 & 86.7 \\
\hline & State & 4,676 & 0 & 51 & 86 & 4,539 & 5.2 \\
\hline & Private & 6,075 & 0 & 335 & 213 & 5,526 & 6.3 \\
\hline & Total & 87,745 & 12 & 490 & 1,073 & 86,171 & 98.2 \\
\hline
\end{tabular}


Table 2. Coal resources reported in millions of short tons, by coal ownership categories for the Southwestern Powder River Basin assessment area. Resource includes coal plus partings. Totals may not sum exactly due to rounding.-Continued

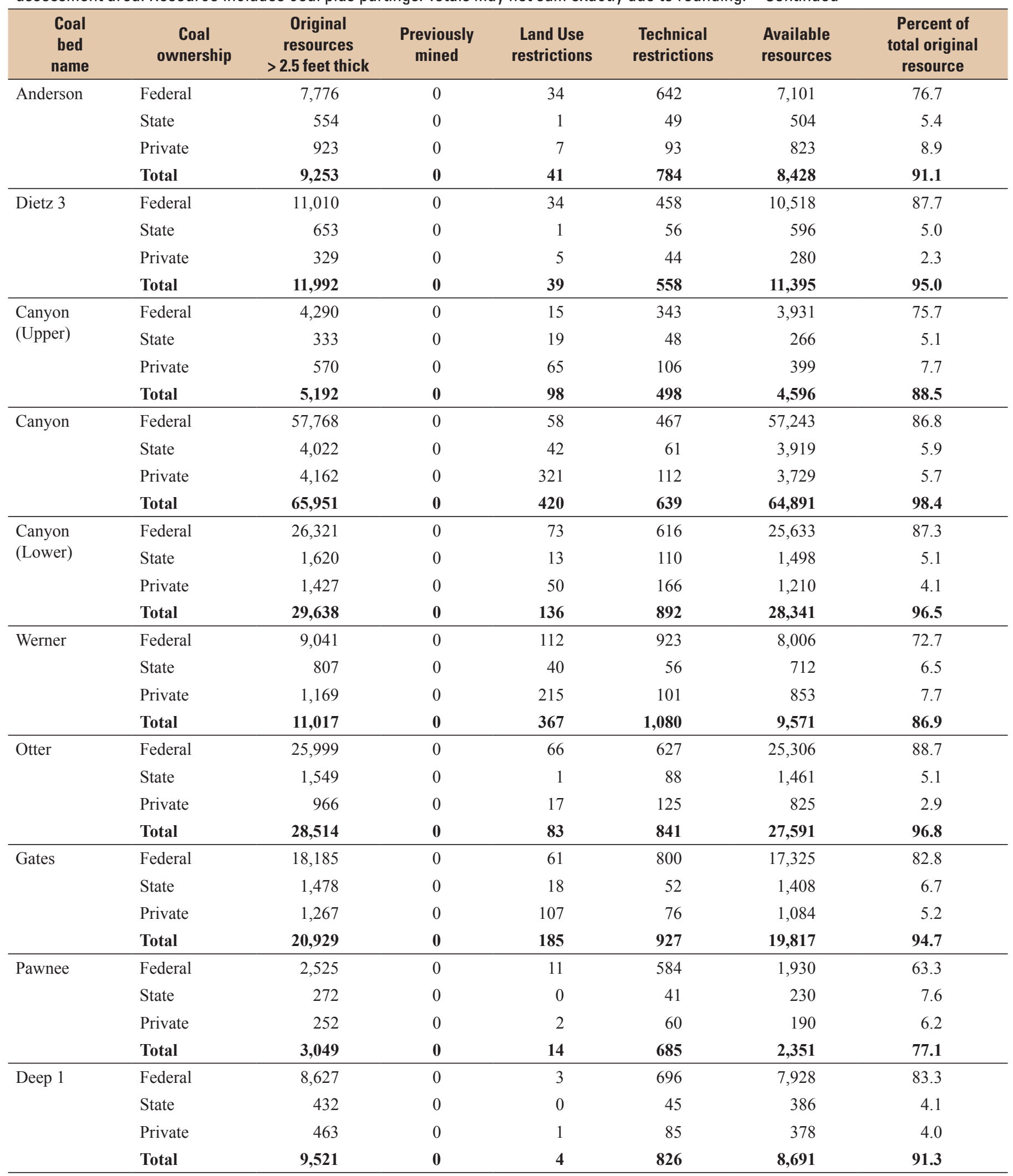




\section{Assessment of Coal Geology, Resources, and Reserves in the Southwestern Powder River Basin, Wyoming}

Table 2. Coal resources reported in millions of short tons, by coal ownership categories for the Southwestern Powder River Basin assessment area. Resource includes coal plus partings. Totals may not sum exactly due to rounding.-Continued

\begin{tabular}{rlrrrrrr}
\hline $\begin{array}{c}\text { Coal } \\
\text { bed } \\
\text { name }\end{array}$ & $\begin{array}{c}\text { Coal } \\
\text { ownership }\end{array}$ & $\begin{array}{c}\text { Original } \\
\text { resources } \\
>\mathbf{2 . 5} \text { feet thick }\end{array}$ & $\begin{array}{c}\text { Previously } \\
\text { mined }\end{array}$ & $\begin{array}{c}\text { Land Use } \\
\text { restrictions }\end{array}$ & $\begin{array}{c}\text { Technical } \\
\text { restrictions }\end{array}$ & $\begin{array}{r}\text { Available } \\
\text { resources }\end{array}$ & $\begin{array}{c}\text { Percent of } \\
\text { total original } \\
\text { resource }\end{array}$ \\
\hline Deep 2 & Federal & 6,346 & 0 & 26 & 1,332 & 4,988 & 66.0 \\
& State & 394 & 0 & 1 & 85 & 308 & 4.1 \\
& Private & 816 & 0 & 23 & 170 & 644 & 8.5 \\
& Total & $\mathbf{7 , 5 5 7}$ & $\mathbf{0}$ & $\mathbf{3 0}$ & $\mathbf{1 , 5 8 7}$ & $\mathbf{5 , 9 4 0}$ & $\mathbf{7 8 . 6}$ \\
\hline Deep 3 & Federal & 11,531 & 0 & 6 & 1,253 & 10,273 & 73.9 \\
& State & 834 & 0 & 0 & 83 & 751 & 5.4 \\
& Private & 1,538 & 0 & 0 & 150 & 1,388 & 10.0 \\
& Total & $\mathbf{1 3 , 9 0 3}$ & $\mathbf{0}$ & $\mathbf{6}$ & $\mathbf{1 , 4 8 6}$ & $\mathbf{1 2 , 4 1 2}$ & $\mathbf{8 9 . 3}$ \\
\hline Roberts & Federal & 9,075 & 0 & 14 & 886 & 8,175 & 72.8 \\
& State & 610 & 0 & 1 & 57 & 553 & 4.9 \\
& Private & 1,544 & 0 & 1 & 80 & 1,464 & 13.0 \\
& Total & $\mathbf{1 1 , 2 2 9}$ & $\mathbf{0}$ & $\mathbf{1 6}$ & $\mathbf{1 , 0 2 2}$ & $\mathbf{1 0 , 1 9 1}$ & $\mathbf{9 0 . 8}$ \\
\hline Total beds & Federal & 314,250 & 12 & 1,937 & 17,078 & 295,223 & 80.0 \\
& State & 23,138 & 0 & 614 & 1,631 & 20,893 & 5.7 \\
& Private & 32,707 & 0 & 3,907 & 2,888 & 24,912 & 6.7 \\
& Total & $\mathbf{3 6 9 , 0 9 5}$ & $\mathbf{1 2}$ & $\mathbf{6 , 4 5 8}$ & $\mathbf{2 1 , 5 9 7}$ & $\mathbf{3 4 1 , 0 2 9}$ & $\mathbf{9 2 . 4}$ \\
\hline
\end{tabular}

Table 3. Coal resources reported in millions of short tons by overburden depth for the Southwestern Powder River Basin assessment area. Resource includes coal plus partings. Totals may not sum exactly due to rounding.

\begin{tabular}{rlrcrrrr}
\hline $\begin{array}{c}\text { Coal } \\
\text { bed } \\
\text { name }\end{array}$ & \multicolumn{1}{c}{$\begin{array}{c}\text { Overburden } \\
\text { thickness } \\
\text { (feet) }\end{array}$} & $\begin{array}{c}\text { Original } \\
\text { resources } \\
\text { > } \mathbf{2 . 5} \text { feet thick }\end{array}$ & $\begin{array}{c}\text { Previously } \\
\text { mined }\end{array}$ & $\begin{array}{c}\text { Land Use } \\
\text { restrictions }\end{array}$ & $\begin{array}{c}\text { Technical } \\
\text { restrictions }\end{array}$ & $\begin{array}{c}\text { Available } \\
\text { resources }\end{array}$ & $\begin{array}{c}\text { Percent of } \\
\text { total original } \\
\text { resource }\end{array}$ \\
\hline Healy (Upper) & $0-500$ & 4,702 & 0 & 824 & 449 & 3,428 & 70.5 \\
& $500-1,000$ & 157 & 0 & 52 & 31 & 74 & 1.5 \\
& $1,000-2,000$ & 6 & 0 & 0 & 6 & 0 & 0.0 \\
& $>2,000$ & 1 & 0 & 0 & 1 & 0 & 0.0 \\
\hline Healy & Total & $\mathbf{4 , 8 6 5}$ & $\mathbf{0}$ & $\mathbf{8 7 7}$ & $\mathbf{4 8 6}$ & $\mathbf{3 , 5 0 2}$ & $\mathbf{7 2 . 0}$ \\
\hline & $0-500$ & 7,536 & 0 & 1,326 & 170 & 6,040 & 64.4 \\
& $500-1,000$ & 1,771 & 0 & 310 & 165 & 1,295 & 13.8 \\
& $1,000-2,000$ & 61 & 0 & 0 & 14 & 47 & 0.5 \\
& $>2,000$ & 5 & 0 & 0 & 1 & 4 & 0.0 \\
\hline Murray & Total & $\mathbf{9 , 3 7 3}$ & $\mathbf{0}$ & $\mathbf{1 , 6 3 6}$ & $\mathbf{3 5 0}$ & $\mathbf{7 , 3 8 7}$ & $\mathbf{7 8 . 8}$ \\
\hline & $0-500$ & 761 & 0 & 18 & 576 & 167 & 11.3 \\
& $500-1,000$ & 662 & 0 & 17 & 428 & 217 & 14.6 \\
& $1,000-2,000$ & 54 & 0 & 0 & 30 & 24 & 1.6 \\
& $>2,000$ & 3 & 0 & 0 & 3 & 0 & 0.0 \\
& Total & $\mathbf{1 , 4 8 1}$ & $\mathbf{0}$ & $\mathbf{3 5}$ & $\mathbf{1 , 0 3 8}$ & $\mathbf{4 0 8}$ & $\mathbf{2 7 . 5}$ \\
\hline Ucross & $0-500$ & 138 & 0 & 39 & 36 & 63 & 1.3 \\
& $500-1,000$ & 4,171 & 0 & 781 & 275 & 3,115 & 62.9
\end{tabular}


Table 3. Coal resources reported in millions of short tons by overburden depth for the Southwestern Powder River Basin assessment area. Resource includes coal plus partings. Totals may not sum exactly due to rounding.-Continued

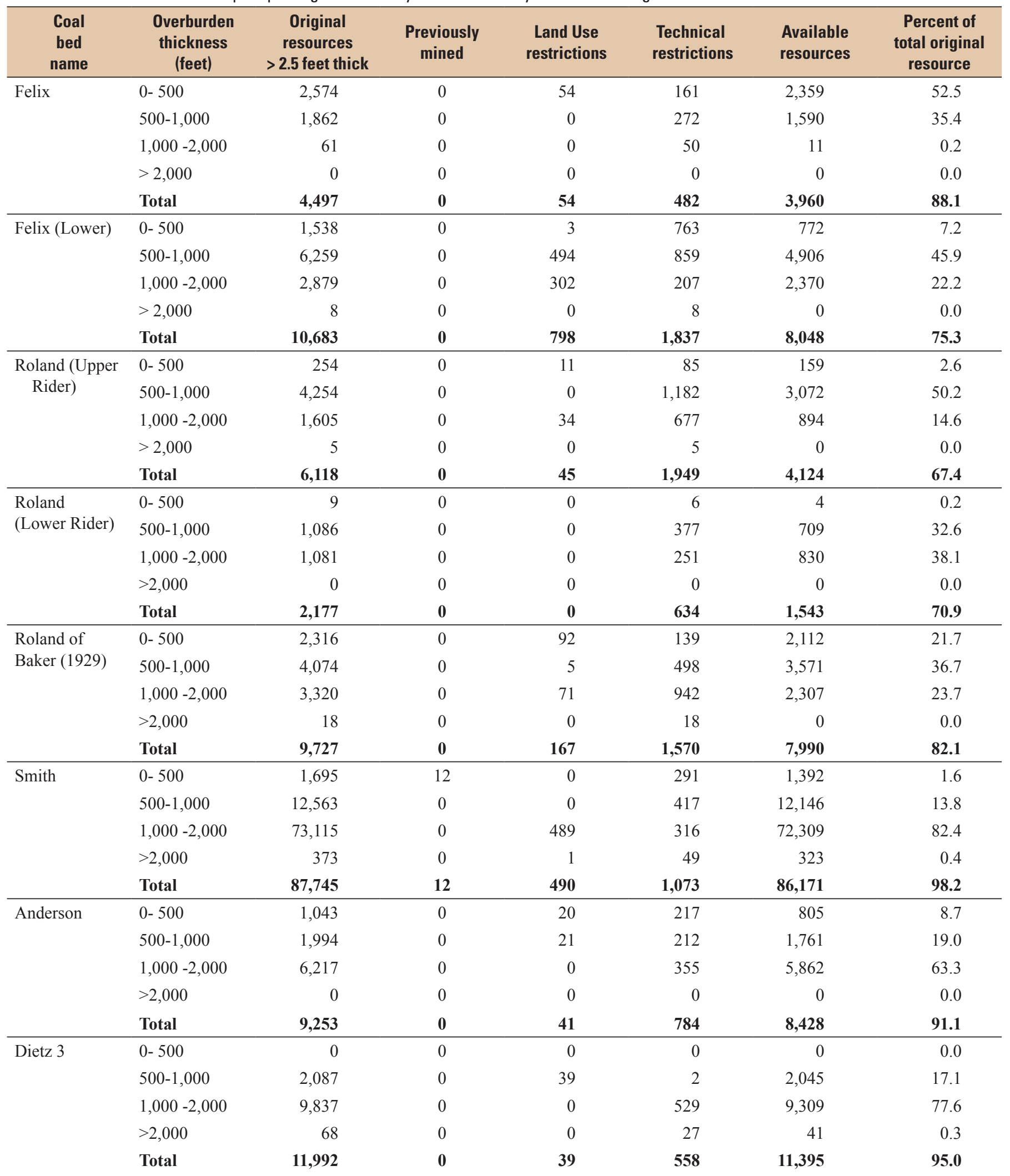


Table 3. Coal resources reported in millions of short tons by overburden depth for the Southwestern Powder River Basin assessment area. Resource includes coal plus partings. Totals may not sum exactly due to rounding._- Continued

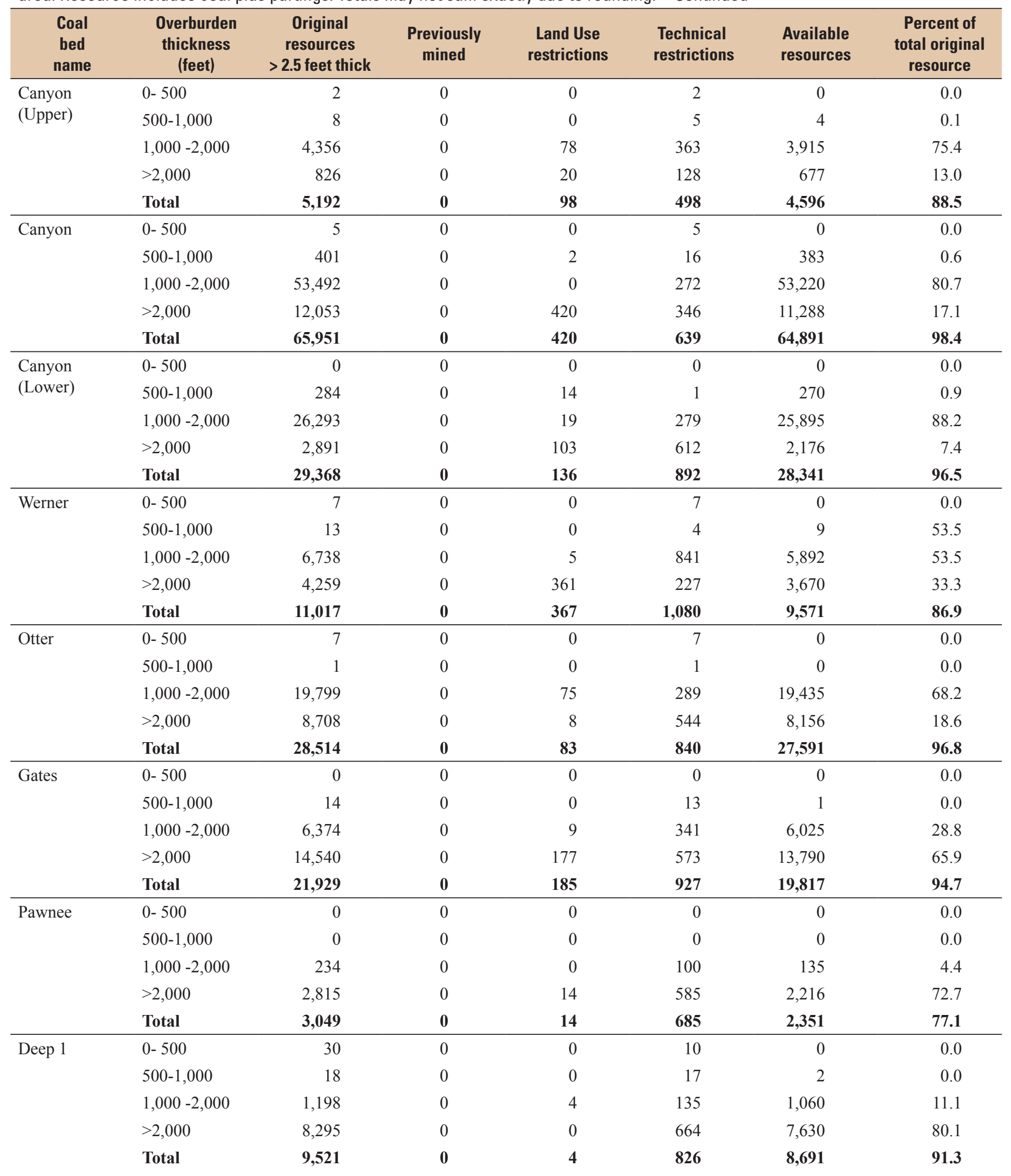


Table 3. Coal resources reported in millions of short tons by overburden depth for the Southwestern Powder River Basin assessment area. Resource includes coal plus partings. Totals may not sum exactly due to rounding.-Continued

\begin{tabular}{|c|c|c|c|c|c|c|c|}
\hline $\begin{array}{c}\text { Coal } \\
\text { bed } \\
\text { name }\end{array}$ & $\begin{array}{l}\text { Overburden } \\
\text { thickness } \\
\text { (feet) }\end{array}$ & $\begin{array}{c}\text { Original } \\
\text { resources } \\
>2.5 \text { feet thick }\end{array}$ & $\begin{array}{l}\text { Previously } \\
\text { mined }\end{array}$ & $\begin{array}{l}\text { Land Use } \\
\text { restrictions }\end{array}$ & $\begin{array}{l}\text { Technical } \\
\text { restrictions }\end{array}$ & $\begin{array}{l}\text { Available } \\
\text { resources }\end{array}$ & $\begin{array}{c}\text { Percent of } \\
\text { total original } \\
\text { resource }\end{array}$ \\
\hline \multirow[t]{5}{*}{ Deep 2} & $0-500$ & 1,148 & 0 & 23 & 216 & 908 & 12.0 \\
\hline & $500-1,000$ & 1,280 & 0 & 6 & 114 & 1,160 & 15.4 \\
\hline & $1,000-2,000$ & 1,549 & 0 & 0 & 343 & 1,205 & 15.9 \\
\hline & $>2,000$ & 3,580 & 0 & 0 & 914 & 2,666 & 35.3 \\
\hline & Total & 7,557 & $\mathbf{0}$ & 30 & 1,587 & 5,940 & 78.6 \\
\hline \multirow[t]{5}{*}{ Deep 3} & $0-500$ & 589 & 0 & 0 & 281 & 307 & 2.2 \\
\hline & $500-1,000$ & 137 & 0 & 2 & 79 & 56 & 0.4 \\
\hline & $1,000-2,000$ & 5,617 & 0 & 4 & 394 & 5,218 & 37.5 \\
\hline & $>2,000$ & 7,561 & 0 & 0 & 730 & 6,831 & 49.1 \\
\hline & Total & 13,903 & $\mathbf{0}$ & 6 & 1,486 & 12,412 & 89.3 \\
\hline \multirow[t]{5}{*}{ Roberts } & $0-500$ & 837 & 0 & 0 & 231 & 605 & 5.4 \\
\hline & $500-1,000$ & 818 & 0 & 7 & 200 & 612 & 5.4 \\
\hline & $1,000-2,000$ & 5,756 & 0 & 4 & 116 & 5,635 & 50.2 \\
\hline & $>2,000$ & 3,818 & 0 & 5 & 474 & 3,339 & 29.7 \\
\hline & Total & 11,229 & $\mathbf{0}$ & 16 & 1,022 & 10,191 & 90.8 \\
\hline \multirow[t]{5}{*}{ Total beds } & $0-500$ & 25,170 & 12 & 2,410 & 3,625 & 19,123 & 5.2 \\
\hline & $500-1,000$ & 43,916 & 0 & 1,750 & 5,169 & 36,997 & 10.0 \\
\hline & $1,000-2,000$ & 230,155 & 0 & 1,191 & 6,893 & 222,072 & 60.2 \\
\hline & $>2,000$ & 69,854 & 0 & 1,107 & 5,911 & 62,837 & 17.0 \\
\hline & Total & 369,095 & 12 & 6,458 & 21,597 & 341,029 & 92.4 \\
\hline
\end{tabular}

Inclusion of a coal bed for this assessment was based on thickness, areal extent, and stratigraphic position in relation to other minable beds. Except for the Upper Healy, Healy, Murray, Ucross, Felix, and Lower Felix coal beds, which are in the Wasatch Formation, the remaining assessed coal beds are all within the Tongue River Member of the Fort Union Formation. Because a typical surface-mining sequence begins at the ground surface, the following descriptions of coal beds that were assessed are discussed from youngest to oldest.

Summary data for the coal bed thicknesses and depths were derived from statistical summaries based on coal bed digital models. Coal resources are classified according to geologic assurances of existence or reliability (Wood and others, 1983). The degree of assurance increases with higher densities of geologic data points. Different reliability categories of measured $(0.25 \mathrm{mi}$ from point of measurement), indicated $(0.25$ $0.75 \mathrm{mi}$ from point of measurement), inferred (0.75-3.0 mi from point of measurement), and hypothetical (greater than $3.0 \mathrm{mi}$ from point of measurement) can be established on the basis of distance from a data point (Wood and others, 1983). Coal resource reliability maps were constructed from the digital model for each coal bed assessed. Nine cross sections were constructed to show the position of coal beds relative to each other (figs. 12-20).

\section{Upper Healy Coal Bed}

The Upper Healy coal bed is the uppermost coal bed in the Wasatch Formation. It is limited in extent to the northwestern part of the SWPRB assessment area and correlates with the Walter coal bed of Mapel (1959). The Upper Healy is also the uppermost bed that comprises the Lake DeSmet coal zone of Obernyer $(1978,1980)$. The Lake DeSmet coal is the one of the thickest known coal zones in the U.S. and is up to $250 \mathrm{ft}$ thick (Mapel, 1959; Obernyer, 1978, 1980). Obernyer $(1978,1980)$ suggested that the Lake DeSmet coal zone at Lake DeSmet (fig. 2) represents the coalescing of several major coal beds, possibly in response to basin-margin faulting active in Eocene time. From youngest to oldest, these beds are the Walters, Healy, Cameron, Murray, and Ucross (Obernyer, 1978, 1980). During this assessment, numerous thick partings were identified in the Lake DeSmet coal zone. During the correlation process in this report, the Lake DeSmet coal zone 
was divided into four major coal beds (Upper Healy, Healy, Murray, and Ucross).

The Upper Healy coal bed, identified on 161 geophysical well logs, has a maximum thickness of $66 \mathrm{ft}$ and an average thickness of $10 \mathrm{ft}$ (figs. 9 and 21). Depth ranges from shallow subcrop to about $1,000 \mathrm{ft}$ along the axis of the basin (fig 22).

The coal resource reliability map is shown in figure 23; approximately 37 percent of the coal resources are in the measured and indicated reliability categories (table 1). As seen in figure 10, much of the Upper Healy coal bed is clinker and would not be considered a resource.

\section{Healy Coal Bed}

Correlated with the Healy coal bed of Mapel (1959), this bed is limited in extent to the northwestern part of the SWPRB assessment area. The Healy coal bed was identified on 449 geophysical well logs, has a maximum thickness of $75 \mathrm{ft}$, and an average thickness of $13 \mathrm{ft}$ (figs. 9 and 24). Over 50 percent of the area of the Healy coal bed thickness exceeds $20 \mathrm{ft}$ with a small area of thickness over $40 \mathrm{ft}$. Depth of the bed ranges from shallow subcrop to about $1,000 \mathrm{ft}$ along the axis of the basin (fig. 25). About 40 percent of the coal resources are in the measured and indicated reliability categories (table 1). The coal resource reliability map is shown in figure 26.

\section{Murray Coal Bed}

The Murray coal bed is also present only in the northwestern part of the SWPRB assessment area. This coal bed correlates with the Murray coal bed of Mapel (1959) and was identified on 593 geophysical well logs, has a maximum thickness of $14 \mathrm{ft}$, and an average thickness of $3 \mathrm{ft}$ (figs. 9 and 27). This coal only attains a thickness of $10 \mathrm{ft}$, south of Buffalo. Depth ranges from shallow subcrop to about $1,000 \mathrm{ft}$ along the axis of the basin (fig. 28). The resource reliability map is shown in figure 29; about 15 percent of the coal resources are in the measured and indicated reliability categories (table 1).

\section{Ucross Coal Bed}

Correlated with the Ucross of Mapel (1959), the Ucross is the bottom coal bed in the Lake DeSmet coal zone of Obernyer $(1978,1980)$ and is limited to the northern part of the SWPRB assessment area. As seen in the isopach maps of the Upper Healy, Healy, Murray, and Ucross coal beds, (figs. 21, 24,27 , and 30 ) the areal extent of all four beds are very similar, suggesting that they are all part of the coal zone comprising the Lake DeSmet coal zone of Obernyer $(1978,1980)$. The Ucross coal bed was identified in 273 geophysical well logs and has a maximum thickness of $38 \mathrm{ft}$ with an average thickness of $9 \mathrm{ft}$ (figs. 9 and 30). One area along the extreme northwestern corner of the SWPRB assessment area contains coal that has a thickness exceeding $20 \mathrm{ft}$. Depth of the Ucross coal bed ranges from shallow subcrop to over $1,000 \mathrm{ft}$ over the area of the thickest part of the coal bed (fig. 31). The resource reliability map for the Ucross coal bed is shown in figure 32 . About 22 percent of the coal resources are in the measured and indicated reliability categories (table 1).

\section{Felix Coal Bed}

The extent of the Felix coal bed is mostly limited to the northeastern part of the SWPRB assessment area. This coal bed, identified in 1,156 geophysical well logs, has a maximum thickness of $48 \mathrm{ft}$ with an average thickness of $7 \mathrm{ft}$ (figs. 9 and 33). One small area contains coal that exceeds a thickness of $20 \mathrm{ft}$. Depth of the Felix coal bed ranges from shallow subcrop to about $1,500 \mathrm{ft}$ over the areas of the thickest part of the coal bed (fig. 34). The Felix coal bed correlates with the Felix coal bed in the NWPRB assessment area (fig. 6). The coal resource reliability map for the Felix coal bed is shown in figure 35 . About 71 percent of the coal resources are in the measured and indicated reliability categories (table 1).

\section{Lower Felix Coal Bed}

The Lower Felix is the lowest coal bed identified in the Wasatch Formation. The bed was identified on 1,890 geophysical well logs, has a maximum thickness of 35 with an average thickness of $7 \mathrm{ft}$ (figs. 9 and 36). The Lower Felix bed is present in a large area in the northern part of the SWPRB assessment area. There are three significant areas where the Lower Felix coal exceeds a thickness of $20 \mathrm{ft}$. Depth of the Lower Felix coal bed ranges from shallow outcrop to about 1,500 $\mathrm{ft}$ along the axis of the basin (fig. 37). The Lower Felix coal bed correlates with the Lower Felix coal bed in the NWPRB assessment area (fig. 6). The coal resource reliability map for the Lower Felix bed is shown in figure 38. Over 26 percent of the coal resources are within the measured and indicated reliability categories (table 1).

\section{Roland (Upper Rider) Coal Bed}

The Roland (Upper Rider) coal bed is an upper split of the Roland (Baker, 1929) coal bed and is most continuous throughout the northern half of the SWPRB assessment area, where it reaches a maximum thickness of $32 \mathrm{ft}$. The bed was identified on 2,805 geophysical well logs and has an average thickness of $4 \mathrm{ft}$ (figs. 9 and 39). This bed, where present, marks the contact between the Wasatch and Fort Union Formations. Depth ranges from shallow subcrop to about $1,500 \mathrm{ft}$ along the axis of the (fig. 40). The Roland (Upper Rider) coal bed correlates with the Roland (Upper Rider) coal bed in the NWPRB assessment area (fig. 6). Approximately 60 percent of the coal resources are within the measured and indicated reliability categories (table 1 ). The coal resource reliability map is shown in figure 41. 


\section{Roland (Lower Rider) Coal Bed}

Where the Roland (Upper Rider) coal bed is absent, the Roland (Lower Rider) is stratigraphically the highest coal bed within the Tongue River Member of the Fort Union Formation. The Roland (Lower Rider) was identified on 1,107 geophysical well logs. With a maximum thickness of $19 \mathrm{ft}$ and an average thickness of $4 \mathrm{ft}$ (figs. 9 and 42) it is less continuous than the Roland (Upper Rider) and is present in a small area in the very northern part of the SWPRB assessment area. Depth ranges from shallow subcrop to about 1,500 $\mathrm{ft}$ along the axis of the basin (fig. 43). The Roland (Lower Rider) coal bed correlates with the Roland (Baker, 1929) coal bed in the NWPRB assessment area (fig. 6). Approximately 58 percent of the coal resources are within the measured and indicated reliability categories (table 1). The coal resource reliability map is shown in figure 44.

\section{Roland of Baker (1929) Coal Bed}

Where neither the Roland (Upper Rider) nor the Roland (Lower Rider) is present, the Roland of Baker (1929) coal bed is stratigraphically the highest coal bed within the Tongue River Member of the Fort Union Formation. In this case, the top of the bed is generally considered to mark the boundary between the Fort Union and Wasatch Formations (Flores and others, 1999) (fig. 9). Hereafter the Roland of Baker (1929) will be referred to as Roland (Baker) coal bed. The bed was identified on 2,442 geophysical well logs. With a maximum thickness of $36 \mathrm{ft}$ and an average thickness of $7 \mathrm{ft}$ (figs. 9 and 45), it is present in a large area in the northern part of the SWPRB assessment area and a smaller area in the southern part of the area. This bed correlates with the Roland coal bed of Luppens and others (2008) in the Gillette coal field and the Roland (Baker) coal bed of Scott and others (2010). The most significant area where the Roland exceeds a thickness of $20 \mathrm{ft}$ is in the extreme northern part of the SWPRB assessment area. Depth of the Roland coal bed ranges from shallow subcrop to over 1,500 ft along the axis of the basin (fig. 46). The Roland of Baker coal bed correlates with the Roland of Taff (1909) coal bed in the NWPRB assessment area (fig. 6). Approximately 65 percent of the coal resources are within the measured and indicated reliability categories (table 1). Coal resource reliability categories for the Roland bed are shown in figure 47.

\section{Smith Coal Bed}

The Smith is the most significant coal bed in the SWPRB assessment area. It was identified on 3,666 geophysical well logs, has a maximum thickness of $266 \mathrm{ft}$, and an average thickness of $32 \mathrm{ft}$ (figs. 9 and 48). It is continuous throughout the northern half of the SWPRB assessment area and correlates to the Smith coal bed of Luppens and others (2008) and the Smith coal bed of Scott and others (2010). The Smith bed is the thickest coal in the SWPRB assessment area where it exceeds $120 \mathrm{ft}$. The coal bed extends eastward into the Gillette coal field where it is one of the more significant coal beds in that field. This coal bed is also equivalent to the Big George coal bed. Depth of the Smith coal bed ranges from shallow subcrop to over 2,500 ft along the axis of the basin (fig. 49). Coal resource reliability categories for the Smith bed are shown in figure 50 . The Smith coal bed correlates with the Smith coal bed in the NWPRB assessment area (fig. 6). Over 84 percent of the coal resources are within the measured and indicated reliability categories (table 1).

\section{Anderson Coal Bed}

Identified on 1,387 geophysical well logs, the Anderson bed has a maximum thickness of $61 \mathrm{ft}$ (figs. 9 and 51). The bed averages $10 \mathrm{ft}$ thick and correlates with the Anderson coal bed in the Gillette coal field and the Anderson coal bed in the NWPRB assessment area (fig. 6). Anderson coal is present in several locations of the SWPRB assessment area, the largest in the central part of the assessment area. Depth of the Anderson coal bed ranges from shallow subcrop to about 2,000 ft along the axis of the basin (fig. 52). More than 73 percent of the coal resources are within the measured and indicated reliability categories (table 1). The coal reliability map is shown in figure 53.

\section{Dietz 3 Coal Bed}

Limited in areal extent to the very northern part of the SWPRB assessment area, the Dietz 3 coal bed was identified on 1,248 geophysical well logs. The bed has a maximum thickness of $119 \mathrm{ft}$ and an average thickness of $14 \mathrm{ft}$ (figs. 9 and 54). There are two small areas where thickness exceeds $40 \mathrm{ft}$. Depth ranges from shallow subcrop to over 2,500 ft along the axis of the basin (fig. 55). The Dietz 3 bed correlates with the Dietz 3 of Scott and others (2010) in the NWPRB assessment area. Depth of the Dietz 3 coal bed ranges from shallow subcrop to over $2,000 \mathrm{ft}$ along the axis of the basin (fig. 56). The Dietz 3 coal bed correlates with the Dietz 1 and Dietz 2 coal beds in the NWPRB assessment area (fig. 6). More than 73 percent of the coal resources are within the measured and indicated reliability categories (table 1).

\section{Upper Canyon Coal Bed}

The Upper Canyon coal bed is an upper split of the Canyon coal bed which is discussed below. Figure 57 shows that the subsurface extent is limited to the northwestern part of the SWPRB assessment area. The coal bed was identified on 396 geophysical well logs. Maximum thickness of the bed is $31 \mathrm{ft}$; average thickness is $8 \mathrm{ft}$ (figs. 9 and 57), and depth ranges from shallow subcrop to about 2,500 ft (fig. 58). Coal resource categories for the bed are shown in figure 59. The 
Upper Canyon coal bed correlates with the Dietz 3 and Dietz 4 coal beds in the NWPRB assessment area (fig. 6). Measured and indicated coal resources total more than 48 percent of the extent of the coal bed (table 1).

\section{Canyon Coal Bed}

Originally named the Monarch coal bed by Taff in1909, this coal bed is equivalent to the Canyon coal bed of Baker (1929) (in Law and others, 1979). Since that time it has been referred to as both the Canyon and the Monarch. In this report it will be referred to as the Canyon coal bed and correlates to the Canyon coal bed in the Gillette coal field (Luppens and others, 2008) and the Canyon coal bed of Scott and others (2010). The Canyon coal bed and the Smith coal bed are the two thickest and continuous coal beds in the SWPRB assessment area. Present throughout most of the northern and central parts of the SWPRB assessment area, the Canyon coal bed was identified in 2,463 geophysical well logs and reaches a maximum thickness of $182 \mathrm{ft}$ with an average thickness of $29 \mathrm{ft}$ (figs. 9 and 60). Depth ranges from shallow subcrop to about 2,500 ft along the axis of the basin (fig. 61). More than 75 percent of the coal resources are within the measured and indicated reliability categories (table 1). The Canyon coal bed correlates with the Canyon coal bed in the NWPRB assessment area (fig. 6). The coal resource reliability map for the Canyon bed is shown in figure 62. Along the eastern edge of the areal extent of the Canyon there is an apparent paleochannel where the coal thins and becomes absent (fig. 60).

\section{Lower Canyon Coal Bed}

Present only in the northern part of the SWPRB assessment area, the Lower Canyon is a lower split of the Canyon coal bed. This coal bed was identified on 1,729 geophysical well logs, has a maximum thickness of $81 \mathrm{ft}$, and averages $16 \mathrm{ft}$ thick (figs. 9 and 63). There are three areas along the eastern edge of the areal extent that exceed $40 \mathrm{ft}$ in thickness. Depth ranges from shallow subcrop to about 3,000 ft along the axis of the basin (fig. 64). More than 73 percent of the coal resources are within the measured and indicated reliability categories (table 1). The Lower Canyon coal bed correlates with the Lower Canyon coal bed in the NWPRB assessment area (fig. 6). The same paleochannel seen in the Canyon coal bed can also be seen in the Lower Canyon coal bed. The coal resource reliability map for the Lower Canyon bed is shown in figure 65.

\section{Werner Coal Bed}

The Werner coal bed, identified in 1,124 geophysical well logs, has a maximum thickness of $76 \mathrm{ft}$ and averages $8 \mathrm{ft}$ thick (figs. 9 and 66). Only small isolated areas contain coal that exceeds $20 \mathrm{ft}$ thick. The Werner coal correlates with the
Werner of Luppens and others (2008) and the Werner of Scott and others (2010). Depth ranges from shallow subcrop to over $2,500 \mathrm{ft}$ over the areas of greatest coal thickness (fig. 67).

More than 47 percent of the coal resources are within the measured and indicated reliability categories (table 1). The Werner coal bed correlates with the Werner coal bed in the NWPRB assessment area (fig. 6). The coal resource reliability map for the Werner bed is shown in figure 68 .

\section{Otter Coal Bed}

Distribution of the Otter coal bed is shown in figure 69 . Based on 1,040 geophysical well logs, it reaches a maximum thickness of $158 \mathrm{ft}$ and has an average thickness of $24 \mathrm{ft}$ (figs. 9 and 69). Areas of greatest thickness occur along the northeastern edge of the SWPRB assessment area. Depth ranges from shallow subcrop to about 4,000 ft along the axis of the basin (fig. 70). More than 71 percent of the coal resources are within the measured and indicated reliability categories (table 1). The Werner coal bed correlates with the Werner coal bed in the NWPRB assessment area (fig. 6). The coal resource reliability map for the Otter bed is shown in figure 71 .

\section{Gates Coal Bed}

The Gates coal bed is slightly larger than the Otter coal bed in areal extent and present only in the northern part of the SWPRB assessment area. The Gates coal correlates with the Gates of Luppens and others (2008) and the Gates of Scott and others (2010). Identified on 819 geophysical well logs, the coal has a maximum thickness of $122 \mathrm{ft}$ and averages $13 \mathrm{ft}$ thick (figs. 9 and 72). Depth ranges from shallow subcrop to about 4,000 $\mathrm{ft}$ along the axis of the basin (fig. 73). More than 49 percent of the coal resources are within the measured and indicated reliability categories (table 1). The Gates coal bed correlates with the Gates coal bed in the NWPRB assessment area (fig. 6). The coal resource reliability map for the Gates bed is shown in figure 74.

\section{Pawnee Coal Bed}

The Pawnee coal bed is present only in small areas in the northern part of the SWPRB assessment area. The coal bed was identified on 217 geophysical well logs, has a maximum thickness of $19 \mathrm{ft}$ and averages $5 \mathrm{ft}$ (figs. 9 and 75). Depth ranges from shallow subcrop to about $4,000 \mathrm{ft}$ along the axis of the basin (fig. 76). The Pawnee coal bed correlates with the Pawnee of Luppens and others (2008) and Scott and others (2010). More than 27 percent of the coal resources are within the measured and indicated reliability categories (table 1). The Pawnee coal bed correlates with the Pawnee coal bed in the NWPRB assessment area (fig. 6). The coal resource reliability map for the Pawnee coal bed is shown in figure 77. 


\section{Deep 1 Coal Bed}

The Deep 1 coal bed, identified on 738 geophysical well logs, has a maximum thickness of $32 \mathrm{ft}$ and averages $9 \mathrm{ft}$ thick (figs. 9 and 78). The coal occurs mostly along the eastern edge of the northern part of the SWPRB assessment area. The Deep 1, Deep 2, and Deep 3 coal beds could not be correlated to any known coal beds. Depth ranges from shallow subcrop to about 4,000 ft (fig. 79). Approximately 57 percent of the coal resources are within the measured and indicated reliability categories (table 1). The coal resource reliability map for the Deep 1 bed is shown in figure 80 .

\section{Deep 2 Coal Bed}

The Deep 2 coal bed occurs along the eastern part of the northern part of the SWPRB assessment area and in a large area in the southern part of the area. Identified on 713 geophysical well logs, the coal has a maximum thickness of $29 \mathrm{ft}$ and averages $5 \mathrm{ft}$ thick (figs. 9 and 81). Depth ranges from shallow subcrop to about $4,000 \mathrm{ft}$ near the axis of the basin (fig. 82). Approximately 36 percent of the coal resources are within the measured and indicated reliability categories (table 1). The coal resource reliability map for the Deep 3 coal bed is shown in figure 83 .

\section{Deep 3 Coal Bed}

The Deep 3 coal bed occurs in about the same areal extent as the Deep 2 coal bed. The coal was identified on 796 geophysical well logs, has a maximum thickness of $32 \mathrm{ft}$, and averages $8 \mathrm{ft}$ thick (figs. 9 and 84). Depth ranges from shallow subcrop to about 4,000 ft near the axis of the basin (fig. 85). Approximately 45 percent of the coal resources are within the measured and indicated reliability categories (table 1). The coal resource reliability map for the Deep 3 coal bed is shown in figure 86.

\section{Roberts Coal Bed}

The Roberts coal was identified in two areas of the SWPRB assessment area (fig. 87). The smaller area is located in the extreme northeast part of the SWPRB assessment area. A larger area is located in the southern part of the SWPRB assessment area. The Roberts coal bed was identified on 465 geophysical well logs, reaches a maximum thickness of $36 \mathrm{ft}$, and averages $9 \mathrm{ft}$ thick (figs. 9 and 87). Depth of the Roberts coal bed ranges from shallow subcrop to over $4,000 \mathrm{ft}$ (fig. 88). Approximately 36 percent of the coal resources are within the measured and indicated reliability categories (table 1). The coal resource reliability map for the Roberts bed is shown in figure 89.

\section{Resource Allocation Planning}

The objective in resource planning is to determine the available resources. This objective is accomplished by first subtracting the resources previously mined from the original resources; the result equals the remaining resources. Subtracting the land use and technical restrictions from the remaining resources equals the available resources. The following discussion details the methodology used in determining available resources.

Geologic digital modeling for the evaluated coal beds was done using PC/Cores (Mentor Consultants, 2005). In the next step of the coal resource assessment, files were imported into a Geographic Information System (GIS). The GIS system was used to allocate coal resources by first subtracting areas with various restrictions to mining (previous mining, towns, railroads, alluvial valley floors, etc.) in order to calculate the amount of available coal.

ArcView $^{\circledR}$ and the ArcView Spatial Analyst ${ }^{\circledR}$ extension (Environmental Systems Research Institute, Inc., 2000, 2001) were used to perform the various GIS analyses and ultimately calculate coal resource volumes. In addition, ArcGIS ${ }^{\circledR}$ (Environmental Systems Research Institute, Inc., 2006) was used in some cases to project digital coverages, shapefiles, or grids to the assessment area's base map projection. The geographic referencing base of the digital data used for the GIS analysis is the Universal Transverse Mercator (UTM) map projection, using the following parameters: map units $=$ meters; zone $=13$; datum=NAD27; and spheroid $=$ Clarke 1866 .

USGS coal resource assessments do not have a standard grid-cell size that is used for all GIS analyses. For this GIS assessment, a grid-cell size of $30 \mathrm{~m}$ (about $100 \mathrm{ft}$ ) on a side was chosen. Consequently, all grids used within the GIS analysis were either originally created with a grid cell size of $30 \mathrm{~m}$ or resampled from $150 \mathrm{~m}$ to $30 \mathrm{~m}$.

The first task in the GIS analysis involved the creation of ArcView (Environmental Systems Research Institute, Inc., 2000) readable grids from the ASCII files of the coal, parting, and overburden isopach grids that were created by the digital coal models. These ArcView grids were used to create a total thickness (coal plus parting) grid and an areal-extent grid for each coal bed to be evaluated. The next task involved creating a group of grids that categorized the SWPRB assessment area, with each grid representing one specific theme. These grid themes consisted of mined out areas, overburden-to-coal bed ratios, counties, coal ownership, resource reliability categories, coal bed depth, and restrictions to mining (that is, land use restrictions and technical restrictions). The grid themes for mined out areas, land-use restrictions, and coal ownership were derived from digital information obtained from the Wyoming Bureau of Land Management. The grid theme for the counties was developed from a digital file of Wyoming county lines obtained from the Wyoming Spatial Data Clearinghouse (2009) (http://wgiac2.state.wy.us). The other grid themes were developed independently for this evaluation. 
The grid of environmental restrictions includes buffer zones that surround the restricted areas. The location and width of these buffers are commonly mandated by State or Federal regulations, but specifications presented in the Buffalo Resource Management Plan (U.S. Bureau of Land Management, 2001), such as additional buffers around the town of Buffalo, Wyoming, were also used in this evaluation. Each restriction buffer measures hundreds or, in the case of the extra-jurisdictional buffer around the town of Buffalo, thousands of feet in width at the surface, as discussed in the following section on factors affecting coal resources.

One of the improvements in the assessment methodology was revising the technique for defining how surface restrictions affect the development of the underlying resources at depth. Previous economic assessments used a simple "cookie cutter" approach when they applied regulatory surface buffers (such as a 300-ft buffer around an inhabited house) below the surface. The surface buffers were simply projected vertically downward through the coal beds to be evaluated. However, a restricted area actually widens with depth when surface mining operations are considered, owing to the additional setback distance required to maintain a safe mining pit highwall angle. Consequently, the area that is affected by a restriction becomes larger the deeper a coal bed lies beneath the surface. For example, a circular restriction at the surface having a diameter of $600 \mathrm{ft}$ encompasses an area of about 282,600 $\mathrm{ft}^{2}$, or approximately 6.5 acres. At a depth of $200 \mathrm{ft}$, however, this same restriction has a diameter of $858 \mathrm{ft}$ encompassing an area of about $577,900 \mathrm{ft}^{2}$, or approximately 13 acres whereas at a depth of $500 \mathrm{ft}$ the restriction has a diameter of 2,144 ft and encompasses an area of about $3,608,400 \mathrm{ft}^{2}$, or approximately 83 acres. Figure 90 illustrates the effect of depth on overall land-use restriction size.

The technical restrictions grids include those areas where coal beds are less than $2.5 \mathrm{ft}$ thick and areas where coal beds are less than $5 \mathrm{ft}$ thick. For this evaluation, coal resources of less than $2.5 \mathrm{ft}$ in thickness are not considered to represent viable resources, whereas coal resources greater than $2.5 \mathrm{ft}$ thick but less than $5.0 \mathrm{ft}$ thick are not considered to represent currently minable resources or potential available resources.

The grid of mined out areas accounted for all previous mining within the SWPRB assessment area, including those mines shown in figure 91. The counties grid contained locations of three principal counties within this area (Campbell, Converse, and Johnson). The coal mineral ownership grid divided the area into Federal, State, and private ownership categories (fig. 92). The resource reliability grid divided the area into measured, indicated, inferred, and hypothetical coal resources whereas the coal bed depth grid divided the area into four categories of depth: 0-500 ft; 500-1,000 ft; 1,000$2,000 \mathrm{ft}$; and greater than 2,000 ft.

Once all the individual theme grids were generated, the next step was to apply these grids to the coal resources of the 23 coal beds to be evaluated. First, the individual theme grids were combined into one composite themes grid, which was then used with the coal bed areal-extent grids to define the SWPRB assessment area resources on a bed-by-bed basis according to all the various categories. Next, areas within each coal bed that represented previously mined coal and restrictions (land-use and technical) were removed from consideration.

\section{Factors Affecting Extraction of Coal Resources}

Many factors can affect the availability of coal for mining. Almost 85 percent of the coal in the SWPRB assessment area is Federally-owned and must be leased in order to be mined (table 2). A four-step coal screening process, defined in the 43 CFR 3420.1-4 regulations, determines which areas of Federal coal are acceptable for leasing. The following list shows coal-leasing unsuitability criteria listed in the Code of Federal Regulations, Title 43 Subpart 3461.5 (43 CFR 3461.5) (Office of the Federal Register, 2003).

\section{Unsuitability Criteria}

Federal land systems

Rights-of-way and easements (for example, railroads)

Dwellings, roads, cemeteries, and public buildings

Wilderness study areas

Lands with outstanding scenic quality

Lands used for scientific study

Historic lands and sites

Natural areas

Critical habitat for threatened or endangered plant and animal species

State listed threatened or endangered species

Bald or golden eagle nests

Bald and golden eagle roost and concentration areas

Federal lands containing active falcon cliff nesting site

Habitat for migratory bird species

Fish and wildlife habitat for resident species

Floodplains

Municipal watersheds

National resource waters

Alluvial valley floors

State or Indian tribe proposed criteria

These 20 specific legal criteria are used to determine if an area is unsuitable for leasing and surface mining. The criteria were established by the Surface Mining Control and Reclamation Act (SMCRA) of 1977 (Public Law 95-87, 1977). The unsuitability criteria require consideration of land use, scenic areas, natural areas, historic sites, wildlife habitats, flood plains, alluvial valley floors, and other special lands. Although the 20 unsuitability criteria were developed for lands owned 
by the Federal government, many of the criteria would also be applicable to State-owned and privately-owned lands. For example, areas containing threatened or endangered plant or animal species are protected from destruction wherever they occur. Municipal watersheds are likewise protected from detrimental actions regardless of who owns the land. It is important to understand that not all criteria listed in the above table affect development within the SWPRB assessment area.

Other potential restrictions to mining include land use restrictions such as; airports, archaeological areas, coalbed methane wells, oil and oil-related gas wells, pipelines, power lines, rivers, lakes, streams, and towns.

Restrictions to mining vary with location and local landmanagement regulations. Thus, different study areas can have different mining restrictions and availability considerations. This report reflects assumptions concerning restrictions to mining that are based on local practices in the PRB, and specifically to those practices within the SWPRB assessment area. In addition, the Bureau of Land Management personnel in Casper, Wyoming, provided guidance concerning restrictions to mining and the distances to be buffered around specific features, and also provided files that delineate many of the features and the buffer distances used for the study. A more detailed determination of restrictions and other availability considerations would be necessary as part of leasing and mineplanning phases of property development.

Figure 91 shows the areas within the SWPRB assessment area that were affected by restrictions. In some cases, an area that was originally declared unavailable for coal mining could have a mitigation measure that would permit mining. The following is a detailed discussion of potential mining restrictions for this area. All buffer restrictions refer to distances at the surface.

\section{Federal Land Systems}

Within the Converse County portion of the SWPRB assessment area, there is a part of the Thunder Basin National Grassland that includes scattered Federal lands under the jurisdiction of the U.S. Forest Service, although it is not part of a National Forest. The same unsuitability criteria and landuse conflicts discussed in this report apply to coal mining on the Thunder Basin National Grassland. Where the mineral ownership there is Federal, the Bureau of Land Management develops coal-leasing and mining stipulations in cooperation with the U.S. Forest Service. Federal subsurface coal ownership is shown in figure 92.

\section{Railroads}

There are three railroad routes through the SWPRB assessment area (fig. 91). The southeast-northwest route in the northern part of the area is a rail line of the Burlington Northern Santa Fe Railway. In the southern part of the area, the north-south route consists of a single railroad corridor combining rail lines of both the Burlington Northern Santa Fe Railway and the Union Pacific Railroad, whereas the generally east-west route consists of two separate rail lines of these same two companies.

Although it is conceivable that the rail lines of these existing routes could be relocated to allow mining to proceed, it is assumed that these main rail lines would not be moved and are restrictions to mining. The total restricted width for each of the railroad lines, including the right-of-way and a 100 - $\mathrm{ft}$ buffer along each side, is $600 \mathrm{ft}$.

\section{Roads}

County roads (mostly gravel) cross many areas throughout the SWPRB assessment area. These roads are not considered to restrict mining because it is assumed that they could be easily relocated or temporarily blocked off to allow mining to proceed. In addition, a number of State and U.S. highways, including Interstate Highways 25 and 90, are present within the area (fig. 91). For this study, it is assumed that all of these, except the interstate highways, could also be relocated to allow for mining. For each of the interstate routes, the total restricted width is $450 \mathrm{ft}$, including the highway right-of-way and a $100-\mathrm{ft}$ buffer on either side.

\section{Dwellings and Buildings}

Individual dwellings and buildings that exist outside of incorporated areas (such as Buffalo) are not considered restrictions to mining within the SWPRB assessment area. These individual structures could probably be purchased by a coal company, which could then move or raze them in order to proceed with mining.

\section{Alluvial Valley Floors}

All areas identified as alluvial valley floors by the State program delegated to enforce the Surface Mining Control and Reclamation Act of 1977 (Surface Mining Control and Reclamation, Public Law 95-87)-where mining would interrupt, discontinue, or preclude farming-are unsuitable for surface coal mining and thus are deemed to be restrictions. In addition, areas outside alluvial valley floors where mining would materially damage the quantity or quality of water supplying alluvial valley floors are unsuitable; however, this analysis did not cover those areas.

\section{Airports}

The Johnson County airport is located near the city of Buffalo, about two and a quarter miles northwest of the central business district and it is included within the Buffalo restriction buffer area. 


\section{Archaeological Areas}

No major archaeological areas that would prevent mining are known in the SWPRB assessment area. There are several minor archaeological sites and also several minor historic sites within this area. A mitigation plan would be developed before coal mining disturbed these areas. Therefore, coal within these known sites was not excluded from this resource study.

\section{Coalbed Methane}

The rapid growth in coalbed methane production within the SWPRB assessment area has resulted in the placement of thousands of wells, along with their accompanying pipeline infrastructure, throughout the area. Designating all of these wells and their extensive gas delivery systems as being restrictive to mining would effectively exclude much of the assessment area from resource consideration. As stated previously, the expected lifetime of these wells in this area, producing from a single coal bed, is 10 to 12 years. The Bureau of Land Management has designated Conflict Administration Zones to minimize conflicts between coal mines and coalbed methane wells in the path of projected near-term mining. For the purpose of this study, it is assumed that coal within any part of the assessment area will be mined after the methane operations have ceased operating in that area. Therefore, these facilities are not considered a restriction to mining.

\section{Oil and Oil-Related Gas Wells}

Hundreds of oil and gas wells are located throughout the SWPRB assessment area. Resolution of land-use conflicts between coal mining and the oil and gas field development will depend on economic conditions, regulations, and negotiations between oil developers and coal developers. An area around a major cluster of active wells might be eliminated from mining activities until these wells are no longer actively producing, or mining activities might proceed around individual active wells that are given a buffer zone. Conversely, specific wells might be plugged and then re-established after mining. For this study, it was assumed that the wells will no longer be actively producing when mining operations reach them and thus they are not considered to be restrictions to mining.

\section{Pipelines}

There is a network of underground oil and gas pipelines throughout the SWPRB assessment area. Probably most, if not all, of these pipelines would be moved so that surface mining could proceed. However, moving and restoring them would represent an added economic cost to mining. In any case, pipelines were not considered to be restrictions to mining for this study.

\section{Power Lines}

All power lines within the assessment area could be moved to accommodate surface mining operations. Thus, power lines are not considered a restriction to mining in this area.

\section{Rivers, Lakes, and Streams}

The Powder River is the longest body of flowing water within the SWPRB assessment area. However, throughout its course within the assessment area, it is a shallow, slow moving, meandering stream as is the case with all of the river's tributaries and other creeks in the SWPRB assessment area. Therefore, surface mining operations could temporarily relocate the courses of these streams and then return them to their pre-mining locations during mine reclamation assuming no protected species of flora or fauna exist. Only the parts of these water courses that have been designated by the State Land Quality Division as alluvial valley floors significant to farming would need to be preserved with no modification. In contrast, although the North Platte River only flows through a small part of the southern end of the assessment area, this prominent water course is considered to be a restriction to mining (fig. 91). In addition to the width of the river, the restriction includes a $100 \mathrm{ft}$ buffer on each side. Shallow lakes and small ponds that do exist could either be temporarily moved during mining or simply reformed after the mining operations ceased. However, Lake DeSmet, a recreational site and water supply source located about six mi north of the city of Buffalo is considered to be a restriction to mining for this assessment (fig. 91). The restriction includes a 100-ft buffer surrounding the lake.

\section{Towns}

The entire municipality of Buffalo and parts of the municipalities of Douglas and Rolling Hills are located within the SWPRB assessment area and are permanent restrictions to mining (fig. 91). In addition to the actual incorporated area, the mining restriction for each municipality includes a buffer that extends well beyond the municipality limits. The boundary of the town buffer for Buffalo is taken from the Buffalo Resource Management Plan Update (U.S. Bureau of Land Management, 2001) developed by the Bureau of Land Management for potential coal development areas within the PRB of Wyoming. The buffers for Douglas and Rolling Hills extend out to a distance of $300 \mathrm{ft}$ beyond each town's limits.

\section{State Lands}

There are no State parks or forests within the SWPRB assessment area. However, the historic site of Fort Reno, a U.S. Cavalry army post constructed in the 1860 s along the 
Bozeman Trail, is located within the SWPRB assessment area and is administered by the Wyoming State Parks and Cultural Resources Department. The site, listed on the National Register of Historic Places, lies near the Powder River approximately 22 mi northeast of the town of Kaycee. The grounds comprising the fort site have mostly returned to a natural cover of prairie sod, although a large stone monument and several interpretive signs mark the site. As is, the site could potentially be a restriction to future mining; however, the site no longer contains original structures or ruins and the area involved is small. Furthermore, the area of the site could be returned to a pre-mining state during remediation and the monument and signs restored. Therefore, for this evaluation there are no State lands that are considered to be restrictions to mining.

\section{Coal Reserve Evaluation Methodology}

The third and final phase in the resource assessment is the economic evaluation of the available resources calculated in phase two (fig. 5). This phase begins by selecting the available resources information for each evaluated bed and parsing them into either surface minable or underground minable resources. However, the potential for available coal resources for coal beds in the Fort Union Wyodak coal zone recovered by surface mining is very limited. On the other hand, significant thick, deep coal resources exist in the Roland (Baker, 1929), Smith, Anderson, Dietz 3, Canyon, Lower Canyon, Werner, and Otter coal beds.

In the extreme southwestern portion of the NWPRB assessment area (Scott and others, 2010) and the northwestern portion of the SWPRB assessment area, there is also a relatively limited area of potentially mineable Wasatch coal beds (Upper Healy and Healy). These coal beds are the northernmost extension of the Buffalo coal field (fig. 2).

Recoverable coal resources are determined through economic analysis. The present value of all mining expenditures over a project life at a rate of return or return on investment is evaluated in a discounted cash flow calculation that yields a discounted cash flow rate of return. A break-even cost per ton is calculated using the discounted cash flow and annual production rate. The portion of the recoverable coal that is minable at or below the current sales price of coal is designated as reserves. Because of the limited surface minable resources, only underground minable resources have been evaluated economically in this report.

\section{Coal Quality}

Coal quality is one of the major factors in the marketability of coal and is an important parameter in economic evaluations as coal is sold based on its calorific value expressed in British thermal units per pound of coal (Btu/ lb). Quality parameters such as increased ash content, which lowers the calorific value, negatively impact the operating and maintenance costs at coal-fired power plants. Therefore, coals with lower ash and sulfur content and higher calorific values command a premium selling price.

The Clean Air Act Amendments have enforced emission limits to reduce certain air pollutants such as sulfur dioxide. The sulfur dioxide emissions limits for all new coal-fired power plants built after 1976 were capped at 1.2 pounds of sulfur dioxide per million Btu (U.S. Environmental Protection Agency, 1980). Any coal that could be burned and meet the sulfur dioxide emission standards for air quality (emit less than 0.6 pounds or less sulfur per million Btu, or 1.2 pounds of sulfur dioxide per million Btu) without the need for flue-gas desulfurization was designated "compliance coal", also known as low sulfur coal (U.S. Energy Information Administration, 2010). Subsequent phases of the Clean Air Act Amendments have further restricted sulfur dioxide emissions. Currently, New Source Performance Standards establish uniform national Environmental Protection Agency air emission standards that limit the amount of pollution allowed from new sources or from modified existing sources (U.S. Environmental Protection Agency, 2005). Under New Source Performance Standards, Best Available Control Technology emission limitations are based on the maximum degree of reduction of each pollutant subject to regulation under the Clean Air Act Amendments (U.S. Environmental Protection Agency, 2005). The minimum Best Available Control Technology standard for sulfur dioxide emissions is 90 percent regardless of the sulfur content. The abundance of low sulfur coal in the PRB has been a significant factor in the steadily growing demand for coal from this region.

There are currently no active mines in the SWPRB assessment area, but limited production from coal beds in both the Fort Union and Wasatch Formations has occurred. Lacking the relatively thick, shallow coal beds found in the Gillette coal field on the eastern flank of the Powder River Basin, mining has not been widespread. As a result, publically available coal quality information from coal exploration coring and mines, except for the now abandoned Dave Johnston Mine near Glenrock, WY, is sparse. Additionally, limited coal quality data have been generated from a few wells during recent coalbed methane exploration. For some coal fields within the SWPRB assessment area, only a single proximate coal analysis for each coal bed was available. For regions like the Gillette coal field where extensive mining with abundant coal analytical data exists, assessing the coal quality variations is straightforward. However, when dealing with relatively few coal analyses, it is especially important to try to assess both the accuracy and representativeness of each available data set.

A review of publically available coal quality data during the current coal resource assessment of the SWPRB assessment area revealed significant problems and other limitations with that data. Problems with accuracy included unaccounted moisture loss and cited data that only presented selective data or introduced errors from the original reports. Problems associated with representativeness are especially difficult to evaluate. If the sample is not representative of the entire coal bed, 
the value of the analytical results on that sample are limited, especially if the sample was biased towards lower ash portions of the bed. When evaluating coal quality data where information is limited, it is always preferable to research the circumstances regarding the sampling and analytical parameters from the original sources. Cited data commonly omits important information such as moisture integrity and representativeness issues.

Luppens (2011) presented a detailed analysis of the published coal quality data available from the SWPRB assessment area. The results of this review for the surface minable portions of coal beds in both the Fort Union and Wasatch Formations are summarized in tables 4 and 5 .

Coal quality for the shallow Fort Union Formation coal beds is from the Dry Cheyenne, Glenrock, and Sussex coal fields (fig. 2, table 4). With the close proximity of Glenrock and Dry Cheyenne coal fields, it would be expected that moisture and apparent rank values would be similar. However, moisture ranges from about 19.5-28.7 percent and correspondingly moist, mineral matter free calorific values range from $8,540-9,805 \mathrm{Btu} / \mathrm{lb}$, which results in a relatively large range of apparent rank from low Subbituminous C to Subbituminous B (American Society for Testing and Materials (ASTM), 2008).

The published average moisture content of 26.6 percent for the produced coal from the Dave Johnston Mine (Glass, 1997), which is owned by Pacific Power and Light Company (PPL), is the most reliable estimate of the moisture content (at 12.1 percent ash) in the Glenrock and Dry Cheyenne coal fields (table 4). However, Glass and Jones (1992) published an average of three analyses from the Dave Johnston Mine that was more than 5.0 percent lower than the published value of (26.6 percent) from the mining company (table 4 ). The three analyses used by Glass and Jones (1992) were originally published by Glass (1975) and consisted of two face channel analyses, 74-36 and 74-37, and a typical core hole analysis provided by mining company from the Smith bed (table 4). The two face channel analyses were obtained from exposed highwall faces that had been exposed to drying conditions for weeks to months prior to sample collection. In fact, the condition of sample 74-36 was described as "weathered" by Glass (1975). The 19.5 percent moisture content of sample 74-37 is more than 7.0-10.0 percent lower than the range in average moisture contents from the adjacent Gillette coal field (Luppens, 2008).

More realistic estimates of ranges in coal quality from thee Glenrock and Dry Cheyenne coal fields would be: moisture, 26.0-29.0 percent; ash, 7.5-12.0 percent; sulfur, 0.4-0.6 percent; and gross calorific values, 7,500-8,000 Btu/lb (Luppens, 2011). These coal quality estimates are significantly lower than would be projected from the published data. It is interesting to note that one of the reasons for closing the Dave Johnston Mine in 2000 was deteriorating coal quality (PACIFICORP, 2008).

The only coal quality data for the Sussex coal field is from a field investigation by Wegemann (1912) (table 4). A few small mines were the source of coal samples for analysis.
Like the Glenrock and Sussex coal fields, the relatively large ranges on the moisture contents and apparent rank are suggestive of effects due to unaccounted moisture loss. Given the lack of impervious materials to seal the samples 100 years ago, it can be assumed that the samples from Wegemann (1912) suffered at least minor moisture loss. The average of the 5 analyses for the Sussex coal field was normalized at 30.0 percent moisture (table 4 ) to derive a more realistic estimate of the expected coal quality of this area. However, the normalized average still falls short of true quality due to the issue of representativeness in the Sussex coal field.

All samples from mines in the Sussex coal field were low ash benches avoiding higher ash partings in each of the mined coal beds (Luppens, 2011). The presence of high ash partings is very common in the Sussex coal field (Wegemann, 1912). It is estimated that whole bed analyses would have ash contents 2.0-5.0 percent and calorific values 200-500 Btu/lb lower that the normalized average (table 4).

Coal quality information for the deep Wyodak-Anderson coal zone resources is especially sparse. An average analysis for the Smith coal bed (Big George) bed at a depth exceeding $1,000 \mathrm{ft}$ is listed in table 4 . Additionally, limited quality information from a few coalbed methane wells was summarized by Stricker (2007). Those data were reviewed by Luppens in 2010 to see if a relationship between depth and calorific values could be established similar to the trend observed by Testa and Pratt (2003). Figure 93 demonstrates that there is a fairly good relationship between the estimated variability calorific values of the Wyodak zone coal beds with depth. Mining costs generally increase with depth. Figure 94 shows that those increased costs at greater depths might be at least partially offset with the realization of increased coal quality, hence higher market value at greater depths.

Limited coal quality data for coal beds in the Wasatch Formation are available. No significant, large-scale mining has occurred in the Wasatch coal beds in the PRB, even though the Healy bed in the Lake DeSmet area (fig. 2) is the thickest known coal bed in the contiguous United States (Jones, 2010). A summary of coal quality data for the Wasatch Formation is presented in table 5. In general, these coal beds have higher ash and sulfur contents and lower calorific values than the Fort Union Formation coals currently being produced in the PRB (Luppens and others, 2008). Partings are common and fairly persistent in many Wasatch coal beds, which probably contribute to the lower quality (Mapel and others, 1953; Jones, 2010).

Limitations with existing published Wasatch Formation coal quality information stem from both the accuracy and, in particular, the representativeness of the data. Mapel and others (1953) noted that the moisture content for the Bureau of Reclamation Core (table 5) was anomalously low (which they attributed to unaccounted for moisture loss during shipping and handling), yet the analytical results were used in calculating the average coal quality for the Lake DeSmet area cores. Given the fact that several face channel samples from mines in the Lake DeSmet area had moistures values in excess of 30.5 percent, all the core samples appear to be at least 1.5 to 
Table 4. Available as-received coal quality data from the Fort Union Formation in the Southwestern Powder River Basin assessment area. (British Thermal Unit per pound (BTU/lb); Moist, mineral-matter free (m,mmf); Subbituminous B (subB); Subbituminous C (subC).

\begin{tabular}{|c|c|c|c|c|c|c|c|c|}
\hline Area name/Coal bed name & $\begin{array}{c}\text { No. of } \\
\text { analyses }\end{array}$ & $\begin{array}{c}\text { Moisture } \\
\%\end{array}$ & $\begin{array}{c}\text { Sulfur } \\
\%\end{array}$ & $\begin{array}{c}\text { Ash } \\
\%\end{array}$ & $\begin{array}{c}\text { Gross } \\
\text { Calorific } \\
\text { Value } \\
\text { (Btu/lb) }\end{array}$ & $\begin{array}{l}\text { Pounds of } \\
\mathrm{SO}_{2} / \text { million } \\
\text { Btu }\end{array}$ & $\begin{array}{c}\text { Gross Calorific } \\
\text { Value }^{7} \\
\text { (Btu/m,mmf) }\end{array}$ & $\begin{array}{c}\text { Apparent } \\
\text { Rank }^{8}\end{array}$ \\
\hline \multicolumn{9}{|l|}{ Dry Cheyenne coal field-Stevens North area $^{3}$} \\
\hline Roland of Baker (1929) coal bed & 5 & 28.7 & 0.51 & 7.7 & 7,831 & 1.30 & 8,540 & subC \\
\hline Smith coal bed & 2 & 26.1 & 0.47 & 7.5 & 8,247 & 1.14 & 8,974 & subC \\
\hline \multicolumn{9}{|l|}{ Glenrock coal field-Stevens area $^{3}$} \\
\hline Roland of Baker (1929) coal bed & 1 & 22.2 & 0.42 & 14.7 & 7,860 & 1.07 & 9,344 & subC \\
\hline Smith coal bed & 1 & 23.5 & 0.38 & 4.4 & 9,010 & 0.84 & 9,460 & subC \\
\hline \multicolumn{9}{|l|}{ Glenrock coal field-Dave Johnston Mine } \\
\hline Roland of Baker (1929) coal bed ${ }^{4}$ & 5 & 27.4 & 0.45 & 7.9 & 7,950 & 1.13 & 8,690 & subC \\
\hline Smith bed face channel sample $74-36^{4}$ & 1 & 20.7 & 0.5 & 15.7 & 7,850 & 1.27 & 9,454 & subC \\
\hline Smith bed face channel sample $74-37^{4}$ & 1 & 19.5 & 0.7 & 8.8 & 8,870 & 1.58 & 9,805 & subB \\
\hline Typical Smith bed core sample analyses from Dave Johnston Mine ${ }^{4}$ & 1 & 26.4 & 0.5 & 9.7 & 7,830 & 1.33 & 8,745 & subC \\
\hline Smith coal bed (average of 3 analyses above from Glass/Jones) & 3 & 22.2 & 0.6 & 11.4 & 8,180 & 1.47 & 9,329 & subC \\
\hline Dave Johnston Mine (produced averages) ${ }^{7}$ & --- & 26.6 & 0.45 & 12.1 & 7,581 & 1.19 & 8,720 & subC \\
\hline \multicolumn{9}{|l|}{ Sussex coal field ${ }^{4,5}$} \\
\hline Reported Value $e^{1,4}$ & 1 & 23.5 & 0.49 & 5.2 & 9,160 & 1.07 & 9,707 & subB \\
\hline $\operatorname{Basin} 2^{1,6}$ & 1 & 28.8 & 0.56 & 6.7 & 7,930 & 1.41 & 8,547 & subC \\
\hline $\operatorname{Basin} 3^{1,6}$ & 1 & 23.6 & 0.68 & 7.8 & 8,450 & 1.61 & 9,228 & subC \\
\hline $\operatorname{Basin} 4^{1,6}$ & 1 & 18.8 & 0.57 & 7.8 & 9,160 & 1.24 & 10,006 & subB \\
\hline
\end{tabular}


Table 4. Available as-received coal quality data from the Fort Union Formation in the Southwestern Powder River Basin assessment area. (British Thermal Unit per pound (BTU/lb); Moist, mineral-matter free (m,mmf); Subbituminous B (subB); Subbituminous C (subC).-Continued

\begin{tabular}{|c|c|c|c|c|c|c|c|c|}
\hline Area name/Coal bed name & $\begin{array}{c}\text { No. of } \\
\text { analyses }\end{array}$ & $\begin{array}{c}\text { Moisture } \\
\%\end{array}$ & $\begin{array}{c}\text { Sulfur } \\
\%\end{array}$ & $\begin{array}{c}\text { Ash } \\
\%\end{array}$ & $\begin{array}{c}\text { Gross } \\
\text { Calorific } \\
\text { Value } \\
\text { (Btu/lb) }\end{array}$ & $\begin{array}{l}\text { Pounds of } \\
\mathrm{SO}_{2} / \text { million } \\
\text { Btu }\end{array}$ & $\begin{array}{c}\text { Gross Calorific } \\
\text { Value }^{7} \\
\text { (Btu/m,mmf) }\end{array}$ & $\begin{array}{c}\text { Apparent } \\
\text { Rank }^{8}\end{array}$ \\
\hline \multicolumn{9}{|l|}{ Sussex coal field ${ }^{4,5}$} \\
\hline Basin $5^{1,6}$ & 1 & 23.5 & 0.49 & 5.2 & 9,050 & 1.08 & 9,590 & subB \\
\hline $\operatorname{Basin} 6^{1,6}$ & 1 & 28.1 & 0.45 & 4.6 & 8,350 & 1.08 & 8,786 & subC \\
\hline Average of 5 Basin Analyses & & 24.6 & 0.6 & 6.4 & 8,588 & 1.28 & 9,228 & subC \\
\hline Estimated Average of 5 Basin analysis (adjusted to $30 \%$ moisture) ${ }^{1}$ & & 30.0 & 0.51 & 6.0 & 7,969 & 1.28 & 8,515 & subC \\
\hline \multicolumn{9}{|l|}{ Deep Basin coal bed } \\
\hline Smith (Big George) coal bed ${ }^{2,8}$ & & 22.6 & 0.25 & 3.5 & 9,750 & 0.51 & 10,135 & subB \\
\hline
\end{tabular}

${ }^{1}$ Partial Bed Sample

${ }^{2}$ Unweighted average of 30 incremental samples from a 200 -ft-thick cored portion of the bed below $1,000 \mathrm{ft}$

${ }^{3}$ Western Fuels Association, Inc., 1983

${ }^{4}$ Glass, 1975

${ }^{5}$ Glass, G.B., and Jones, R.J.(1992)

${ }^{6}$ Wegemann, C.H. (1912)

${ }^{7}$ Glass (1997)

${ }^{8}$ Jones (2010) 
Table 5. Available as-received coal quality data from the Wasatch Formation in the Lake DeSmet area in the Southwestern Powder River Basin assessment area. (British Thermal Unit per pound (BTU/lb); Moist, mineral-matter free (m,mmf); Subbituminous B (subB); Subbituminous C (subC); Lignite A (ligA).

\begin{tabular}{|c|c|c|c|c|c|c|c|c|c|c|c|c|c|c|c|}
\hline \multirow{2}{*}{ Area/Coal bed name } & \multirow{2}{*}{$\begin{array}{l}\text { No. of } \\
\text { analy- } \\
\text { ses }\end{array}$} & \multicolumn{2}{|c|}{$\begin{array}{l}\text { Coal bed } \\
\text { interval }\end{array}$} & \multicolumn{2}{|c|}{$\begin{array}{l}\text { Analytical } \\
\text { interval }\end{array}$} & \multirow{2}{*}{$\begin{array}{c}\text { Total } \\
\text { interval } \\
\text { cored }\end{array}$} & \multirow{2}{*}{$\begin{array}{c}\text { Total } \\
\text { feet } \\
\text { analyzed }\end{array}$} & \multirow{2}{*}{$\begin{array}{c}\text { Recov- } \\
\text { ery } \\
\%\end{array}$} & \multirow{2}{*}{$\begin{array}{c}\text { Mois- } \\
\text { ture } \\
\%\end{array}$} & \multirow{2}{*}{$\begin{array}{c}\text { Sulfur } \\
\%\end{array}$} & \multirow{2}{*}{$\begin{array}{c}\text { Ash } \\
\%\end{array}$} & \multirow{2}{*}{$\begin{array}{c}\text { Calorific } \\
\text { value } \\
\text { (Btu/lb) }\end{array}$} & \multirow{2}{*}{$\begin{array}{l}\text { Lbs. of } \\
\mathrm{SO}_{2} \text { per } \\
\text { million } \\
\text { Btu }\end{array}$} & \multirow{2}{*}{$\begin{array}{c}\text { Calorific } \\
\text { value } \\
\text { (Btu/lb } \\
\text { m,mmf) }\end{array}$} & \multirow{2}{*}{$\begin{array}{l}\text { Apparent } \\
\text { rank }\end{array}$} \\
\hline & & From & To & From & To & & & & & & & & & & \\
\hline Lake DeSmet area & 15 & & & & & & & & & & & & & & \\
\hline Healy coal bed ${ }^{1}$ & 1 & & & & & & & & 28.5 & 0.6 & 7.6 & 7,880 & 1.52 & 8,583 & subC \\
\hline USGS-1 Core ${ }^{1}$ & & 59.0 & 141.7 & 59.0 & 141.7 & 82.7 & 45.5 & 55.0 & 29.1 & 0.6 & 5.1 & 7,970 & 1.51 & 8,432 & subC \\
\hline USGS-2A Core $^{1}$ & & 66.0 & 119.0 & 66.0 & 119.0 & 53.0 & 40.8 & 77.0 & 30.5 & 0.5 & 6.1 & 7,845 & 1.27 & 8,396 & subC \\
\hline USGS3 Core ${ }^{1}$ & & 101.0 & 159.7 & 118.7 & 159.7 & 58.7 & 37.2 & 63.4 & 28.3 & 0.6 & 7.7 & 7,890 & 1.52 & 8,604 & subC \\
\hline USGS-6 Core ${ }^{1}$ & & 172.0 & 285.0 & 172.0 & 272.0 & 113.0 & 68.1 & 60.3 & 29.3 & 0.6 & 9.2 & 7,515 & 1.60 & 8,341 & subC \\
\hline Bureau of Reclamation Core ${ }^{1}$ & & 95.0 & 224.0 & 95.0 & 224.9 & 129.0 & 101.6 & 78.7 & 23.6 & 1.0 & 9.7 & 8,270 & 2.42 & 9,239 & subC \\
\hline $\begin{array}{l}\text { Weighted average of all } 5 \text { core } \\
\text { holes }\end{array}$ & 5 & & & & & & & & 27.0 & 0.7 & 8.1 & 7,940 & 1.76 & 8,700 & subC \\
\hline $\begin{array}{l}\text { Estimated average of } 5 \text { basin } \\
\text { analyses (at } 30.5 \% \text { moisture) }\end{array}$ & 5 & & & & & & & & 30.5 & 0.7 & 7.7 & 7,559 & 1.76 & 8,243 & $\operatorname{ligA}$ \\
\hline $\begin{array}{l}\text { Healy coal bed (face channel } \\
\text { mine sample) }\end{array}$ & 1 & & & & & & & & 30.7 & 0.4 & 5.1 & 7,900 & 1.01 & 8,359 & subC \\
\hline $\begin{array}{l}\text { Bed } 120 \text { feet below Healy } \\
\text { coal bed (face channel mine } \\
\text { sample) }\end{array}$ & 1 & & & & & & & & 31.1 & 1.4 & 8.8 & 7,350 & 3.81 & 8,114 & $\operatorname{ligA}$ \\
\hline
\end{tabular}


3.0 percent low. Therefore, the average values for the core samples in table 5 were adjusted to a more realistic 30.5 percent (Luppens, 2011).

Unlike the moisture issue, the representativeness of the data is more difficult to evaluate due to a combination of incomplete core recovery and, specifically, the exclusion of all high ash intervals greater than 3/8 inch in thickness for all USGS core samples. U.S. Geological Survey guidelines at the time mandated that their samplers strictly followed the exclusionary procedure for partings and pyrite of certain thicknesses (Golightly and Simon, 1989). The percent of the coal bed analyzed recovery ranged from about 57 to 78 percent (table 5). The total amount of shale partings excluded from the recovered core ranged from $1.1-33.8 \mathrm{ft}$ and averaged $12.8 \mathrm{ft}$ or about 14 percent of the total recovered core. Therefore, the core hole analytical results cannot be considered even close to being representative, especially with all high ash and pyrite partings excluded.

Core hole USGS-1 had a percent of excluded shale to total recovered core that was similar to that of the 5 core holes (table 5). Luppens (2011) used this core hole to derive an estimate of the weighted composite coal quality of the coal with the excluded shale. The results of this compositing effort were ash contents that ranged from about 13.0-15.5 percent and gross calorific values from 6,700-7,000 Btu/lb. This composite estimate more than doubles the average ash content from the original reported for USGS-1 of 5.1 percent (table 5) with a corresponding penalty to the gross calorific value. Additionally, the effects on the average sulfur content of the composited estimates were impossible to assess due to a lack of data but intuitively, the sulfur content for the whole bed should be higher than reported with deliberate exclusion of the black shale and pyrite-rich partings.

The thorough review of the original report for the Lake DeSmet area resulted in a projected average coal quality that was significantly lower than the published data. With the relatively high ash and sulfur contents combined with low gross calorific values, the estimated coal quality of the area is not competitive with any other coal currently being produced in the Powder River Basin.

The previous discussion demonstrates why it is always preferable to research and evaluate the circumstances regarding the sampling and analytical results from the original source when evaluating coal quality information, especially when only limited data are available. Cited data often omit important information such as moisture integrity and the information needed to evaluate the issue of representativeness. Using the available published data at face value would have significantly over-estimated the coal quality for all the coal fields from both the Fort Union and Wasatch Formations in the SWPRB assessment area. However, if sufficient sampling and handling information is provided, it is often possible to make adjustments to reported data to derive more reasonable coal quality estimates.

\section{Mine Model Design}

Mine models have been developed to evaluate multiple and single coal bed resources in the Powder River Basin for their potential as reserves. The model included only a single pass longwall underground mining model. Each model is developed to produce a conceptual costing estimate that includes identification of primary capital expenditures and operating costs. The models are integrated with economic analyses that identify scheduled expenditures for equipment and facilities replacement, depreciation in accordance with current Internal Revenue Service guidelines, estimate accuracy in terms of cost contingency, and a typical return on investment. The economic model is used to determine the total costs for the term of the designated project life and the total mass of coal mined during this period and by the methodology through which the costs were generated. The calculated result is a break-even cost per short ton of mined coal. The breakeven cost is the calculated mid-point: values above the mid-point denote profitability and values below the mid-point denote economic loss. The models are structured to provide instantaneous results from adjustments to key parameters and thereby provide a tool for comparisons among operating alternatives.

\section{Economic Analysis Methodology}

The breakeven cost per ton is determined through discounted cash flow rate of return analysis. Annual cash flow, the difference between income and expense per year, is modified in this analysis to include only the expense component. Income is determined from the amount needed to recover the total of all yearly expenses at a rate of return for a project life that is expressed in years. The rate of return can vary from year to year or over the span of several years, but in this analysis the rate of return is set at 8 percent (T.K. Lee, written commun., 2004). The project life is set at 20 years, a common term or span over which capital and operating items are expended. All expense values are expressed in present worth or current dollar values.

Capital expenditures are made prior to the infusion of income into a project. The convention used in this report combines all expenditures into an initial cost made at project year 0 . Year 0 , whenever it is assigned, is the moment at which expenditures are expressed in current dollar values. This report is produced in calendar year 2010 and project year 0 is 2010 . Indirect capital cost and similar expenses are applied as a percentage of the initial capital investment for lease acquisition, engineering, home office overhead when applicable, and similar expenses. An amount of 9 percent is added as indirect cost (engineering, construction management and overhead, working capital, and so forth). Amortization and salvage are excluded in this evaluation.

Subsequent capital expenditures for equipment and facility replacement are made in various project years $(5,7,10,14$, 15 , and so forth) to account for wear and obsolescence, and 
are then discounted using the Single Payment Present Worth calculation to project year 0 to show their true present worth. This calculation may be expressed as: $1 /(1+\mathrm{i})^{\mathrm{n}}$ where "I" is the interest rate and " $n$ " is the number of compounding periods. As these analyses are performed on an annual basis, " $n$ " equals one for each year. This calculation is used to discount a single future cost or income value to a single present value. Time-value discounting is also applied to annual operating costs, which is determined using the Uniform Series Present Worth calculation and to depreciation. The Uniform Series Present Worth formula is shown as: $\left[(1+\mathrm{i})^{\mathrm{n}}-1\right] / \mathrm{I}$. This calculation is used to discount a series of uniform future values to a single present value.

All equipment and facilities have been depreciated as per Internal Revenue Service guidelines (Department of the Treasury, 2008). The convention used herein is to depreciate capital items as quickly as allowable - that is, 5- and 7-yr class life capital items use the 200 percent declining balance method, 15- and 20-yr class life capital use the 150 percent declining balance method, and 39-yr class life capital uses the straight line method. Annual depreciation amounts for each of the class life designations are calculated using standard percentages provided in the Modified Accelerated Cost Recovery System General Depreciation System and totaled for each year of the 20-yr project life (Department of the Treasury, 2008).

Some key equipment items may not be replaced during the project life and will continue in service past the designated depreciation period. These items in the surface and underground mining models have been depreciated nonetheless in accordance with IRS Publication 946 (Department of the Treasury, 2008). The amount for each year was discounted to a total present value. When the major expenditures have all been discounted to present dollar values, the break-even cost per ton mined is calculated from the major costs as shown in table 6 . The costs shown in table 6 represent the Case 1 evaluation described later in this report.

Total values shown in table 7 represent the expenditures over an economic project life of 20 years. The total capital cost including initial and replacement capital expenditures equals the total cash flow in this calculation and can be reversed bottom to top to determine the required capital expenditure to support a fixed cost and production level. Exclusions to the above calculation are depletion allowance, salvage, and working capital. These charges over a long project life are minor and are included within the cost contingency. Estimation of expenditures is performed at a high level in this report to keep the order-of-magnitude estimates.

Cost estimates performed for this report are conducted at a conceptual feasibility level corresponding with a Class IV or 4 estimates as defined by the Association for the Advancement of Cost Engineering (AACE, 1997) as shown in table 8. A contingency is usually applied to all cost estimating practices. The cost contingency is determined by the level of project definition, preparatory effort, and whether a judgment, stochastic estimating, or definitive estimating method is used.
Contingency is calculated as a percentage of the estimated costs and added to the estimated costs to give the total project cost.

The level of contingency is also related to time and type of industry. Association for the Advancement of Cost Engineering (AACE, 1997) contingency guidelines for estimate accuracy usually pertain to construction and general applications and have been adjusted in table 8 , which represents values commonly used in the mining industry. Normally, as a mining project moves from inception to construction and subsequent production, the accuracy percentage range decreases with the inclusion of additional information.

\section{Underground Mine Model Assumptions}

An underground mining model was developed to evaluate the potential for extraction from eight deep coal beds including the Roland (Baker), Smith, Anderson, Dietz 3, Canyon, Lower Canyon, Werner, and Otter (figs. 45, 48, 51, 54, 60, 63, 66 , and 69). Large areas within all these coal beds are 10-20 ft thick with much greater thicknesses of 60-100 ft in the Anderson, Canyon, and Otter coal beds. Locally, the Smith coal bed thickens to as much as $150 \mathrm{ft}$ (fig. 48). The resources for the eight coal beds are all within the mineable depth of 2,000 ft from the surface, and thus all are potential resources.

The underground mining of very thick coal beds has not been a practice within the United States although, thick underground coal beds have been mined elsewhere by a variety of methods. Conventional longwall mining technology was chosen as the method of choice in this report. Current face shearer and roof prop designs are available for mining to a maximum thickness of $20 \mathrm{ft}$.

The underground mine model is based on a typical single pass longwall mining methodology that can produce a nominal 6 MST of coal per year, which is an average size for currently operating longwall mines (fig. 94). The model has the flexibility to allow for varying mining thicknesses from 8-20 ft and depths of less than 1,000-2,000 ft. The model can be used to evaluate a wide range of annual production rates with some adjustment to longwall panel access openings and pillar sizes.

The mining model was designed for primary entry to the longwall panel through five parallel main drifts. Additional excavation included a three entry headgate for incoming airway and main access to longwall mining and two entry tailgates for return airway and supplies. Both the headgate and tailgate openings extend perpendicular from the main entries to the full panel length and are separated by the width of the longwall panel. An initial longwall face opening is excavated at the far end of the longwall panel that connects the headgate and tailgate. Excavation dimensions for the mining model are shown in table 9.

A longwall unit in the underground mine model includes five parallel main drifts, a three entry headgate, a two entry tailgate, a longwall face, and a panel. All mains, including headgate and tailgate entries, are the standard $10 \mathrm{ft}$ high 
Table 6. Costs by underground mining case for the Southwestern Powder River Basin assessment area based upon a 20-year project life at an annual production rate of 6 million tons per year. All major costs are shown in mid calendar year 2010 dollar values and breakeven costs in dollars per ton.

\begin{tabular}{lrrrr}
\hline & Case 1 & Case 2 & Case 3 & \multicolumn{1}{c}{ Case 4 } \\
\hline Mining height & $10 \mathrm{ft}$ & $20 \mathrm{ft}$ & $10 \mathrm{ft}$ & $20 \mathrm{ft}$ \\
Depth & $1,000 \mathrm{ft}$ & $1,000 \mathrm{ft}$ & $2,000 \mathrm{ft}$ & $2,000 \mathrm{ft}$ \\
Capital cost & $\$ 385,600,000$ & $\$ 375,900,000$ & $\$ 408,600,000$ & $\$ 400,900,000$ \\
Depreciation & $\$ 167,900,000$ & $\$ 164,700,000$ & $\$ 172,700,000$ & $\$ 168,900,000$ \\
Operating cost & $\$ 3,218,500,000$ & $\$ 3,166,800,000$ & $\$ 3,276,200,000$ & $\$ 3,227,300,000$ \\
Breakeven cost per ton & $\$ \mathbf{3 0 . 9 4}$ & $\mathbf{\$ 3 0 . 4 0}$ & $\$ \mathbf{3 1 . 6 9}$ & $\$ \mathbf{3 1 . 2 0}$ \\
\hline
\end{tabular}

Table 7. Costs of underground longwall mining for the Southwestern Powder River Basin assessment area based on a 20-year project life at an annual production rate of 6 million tons. All costs are shown in mid calendar year 2010 dollar values and breakeven cost in dollars per ton.

\begin{tabular}{lr}
\hline \multicolumn{1}{c}{ Component } & \multicolumn{1}{c}{ Cost } \\
\hline Capital cost & $\$ 385,600,000$ \\
Depreciation & $\$ 167,900,000$ \\
Operating cost & $\$ 3,213,000,000$ \\
Breakeven cost per ton & $\$ \mathbf{3 0 . 9 4}$ \\
\hline
\end{tabular}

Table 8. Contingency alternatives for estimating costs for longwall mining.

\begin{tabular}{llcc}
\hline \multicolumn{1}{c}{ Type } & \multicolumn{1}{c}{ Methodology } & Level of Detail & Accuracy range \\
\hline Order of Magnitude & Judgment or stochastic & $0-2 \%$ & Up to $50 \%$ \\
Conceptual & Stochastic & $1-15 \%$ & $+30 /-15 \%$ \\
Budgetary & Mixed stochastic and deterministic & $10-40 \%$ & $+15 /-10 \%$ \\
Definitive & Deterministic & $50-100 \%$ & $+10 /-5 \%$ \\
\hline
\end{tabular}

Table 9. Underground excavation dimensions in the longwall mining model for a 10-foot mining height.

\begin{tabular}{lcccc} 
& Number & Height (feet) & Width (feet) & Length (feet) \\
\hline Main entries & 5 & 10 & 24 & 1,945 \\
Main crosscuts & 94 & 10 & 18 & 85 \\
Headgate & 3 & 10 & 18 & 11,635 \\
Headgate crosscuts & 131 & 10 & 18 & 65 \\
Tailgate & 2 & 10 & 16 & 11,635 \\
Tailgate crosscuts & 66 & 10 & 16 & 65 \\
Longwall entry & 1 & 10 & 18 & 1,000 \\
\hline
\end{tabular}


regardless of the mining height $(8-10 \mathrm{ft})$. Opening widths are $24 \mathrm{ft}$ for the mains, $18 \mathrm{ft}$ for the headgate, and $16 \mathrm{ft}$ for the tailgate. Crosscuts are $18 \mathrm{ft}$ wide for the mains, headgate, and tailgate. Pillars in the mains are 65 by $85 \mathrm{ft}$ and the chain pillars in the headgate and tailgate are 65 by $160 \mathrm{ft}$. The panel barrier pillar varies from 160 to $300 \mathrm{ft}$ wide, depending on mining height and depth. Only the panel barrier pillar is adjusted for depth. Other pillars in the panel development have been oversized. A panel width of $1,000 \mathrm{ft}$ is used for coal having a $10 \mathrm{ft}$ mining height and $500 \mathrm{ft}$ for a $20 \mathrm{ft}$ mining height.

Annual production for the underground mine model is the sum of coal mined from main access, headgate and tailgate excavation, longwall production, and pillar recovery. Physical dimensions of the longwall panel and associated mine openings are adjusted to maintain 6 MST per year. The effects of increased mining height and depth have been correlated with variable panel barrier pillar size and overall pillar recovery. The longwall panel width varies only with mining height and the panel length is adjusted to provide an overall annual production from entries, panel, and pillars of or near six million tons.

Underground mining requires both surface and subsurface support facilities and mining system, which is identified as the longwall unit. Expenditures for surface support facilities include land improvements, buildings, other facilities (electrical distribution, natural gas service, fuel storage, and water treatment), plant site equipment, hoisting plant, ventilation plant, and coal handling. Only costs associated with hoisting, ventilation, and water handling are appreciably changed with variable mining depths. Underground support facilities include shaft and ramp construction and furnishing, shaft and ramp station construction and equipment, underground ventilation, and all non-surface coal handling. The main drifts excavated from the shaft and ramp stations to the limit of the shaft-ramp barrier pillar are considered pre-development and expensed as a capital cost. All coal derived from pre-development is disposed at the surface. Variations in mining depth affect shaft, ramp, inclined conveyor, ventilation, and electrical distribution costs. A slight adjustment is applied for electrical distribution, chiefly for increased electrical cable size. The longwall unit includes all longwall face and entry development equipment needed to excavate and maintain underground openings previously described.

\section{Resources Evaluation}

Original resources were calculated for all coal beds more than $2.5 \mathrm{ft}$ thick and with no restrictions taken out. Remaining resources were calculated using the volume of the original resource minus the volume of coal that had been mined. Land use (environmental) and technical restrictions were then calculated and subtracted from the remaining resource to determine available resources.

The 23 coal beds evaluated in the SWPRB assessment area are calculated to contain 369 BST of original coal resources (tables1, 2, and 3). The majority of the available resources, 295 BST ( 80 percent of the total), are on Federal lands (table 2). About 12 MST of the total original coal resource have already been mined, and approximately 16 BST are affected by restrictions (land use, and technical). The subtraction of all the restrictions to mining leaves $341 \mathrm{BST}$ (92.4 percent) of the total original coal resource available for development in the SWPRB assessment area.

Coal resource reliability categories for the original coal resources for the 23 assessed coal beds are summarized in table 1. About 65.7 percent of the total original resources can be classified as measured and indicated, reflecting the overall substantial improvement in geologic confidence provided by the additional data included in this assessment.

\section{Surface Coal Economic Analyses Results}

Some of the key geological criteria used to evaluate the potential for surface mining of a coal bed are: thickness, depth (overburden thickness), areal extent, structural complexities, data distribution, and coal quality. Since there are differences in the coal geology between the Fort Union and Wasatch Formations, each formation is discussed separately.

\section{Fort Union Formation}

There are significant changes in the basic coal geology of the Wyodak-Anderson coal zone moving from the Gillette coal field (Luppens and others, 2008) to the NWPRB assessment area (Scott and others, 2010) and to the SWPRB assessment area. The most notable change is the thinning and splitting of the deep, thick Wyodak zone towards the southern and western flanks of the PRB. This thinning can be readily observed in the cross sections (figs. 12-20) and the coal bed isopach maps (figs. 45, 48, 51, 54, 60, and 63). Additionally, the cross sections (figs. 12-20) illustrate that the Wyodak zone coal beds are thin and (or) pinch out throughout much of the SWPRB assessment area before the beds reach shallower depths where recovery by surface mining would be practicable. Relatively steep dips $\left(10^{\circ}-25^{\circ}\right)$ of any coal beds on the western flank of the PRB such as the Sussex coal field are also a limiting factor (fig. 2).

The composite stripping ratio map for the Fort Union coals is shown in fig. 10. The only shallow resources at less than a 10:1 stripping ratio are a narrow band of the Roland (Baker) and Smith coal beds in the southern portion of the SWPRB assessment area which coincides with the abandoned Dave Johnston Mine. Figure 11 illustrates the geographic setting for this area with less than 10:1 stripping ratio. This area lies on the axis of the PRB with no other Fort Union Formation coal beds present. The relationship between the Smith coal bed in the Glenrock coal field and the Powder River Basin structural axis is shown on overburden depth map for the Smith coal bed (fig. 49). The coal reliability maps for the Roland (Baker) and Smith coal beds (figs. 47 and 50) 
demonstrate the lack of sufficient drilling to adequately assess the surface recoverable resources left in the Glenrock coal field (fig. 2).

Associated with the thinning and splitting of the coal beds is the presence of more numerous high ash partings that negatively impacted the coal quality by increasing the ash content and lowering the calorific values. The potential for even lower grade coal quality was a factor in the decision to close the Dave Johnston Mine, the only large commercial mine in the SWPRB assessment area. Closure of this mine reflects the trend of decreasing coal quality along the shallow southern and western flanks of the SWPRB assessment area. The combined effects of the lack of extensive, thick, and shallow coal bed exploration targets with attractive coal quality has resulted in a lack of sufficient drilling (both conventional coal exploration holes and coalbed methane development). Sufficient drilling is necessary to adequately assess surface recoverable coal resources of the SWPRB assessment area on a regional basis. Therefore, no economic analysis of the surface recoverable Fort Union Formation coals (Roland of Baker (1929) and Smith) was performed for the assessment of the SWPRB assessment area.

\section{Wasatch Formation}

The Buffalo-Lake DeSmet area (fig. 2), near Buffalo and Lake DeSmet, contains Wasatch Formation coals that have been evaluated a number of times in the past for potential mining (J.A. Luppens, oral commun., 2010). Despite the fact that the Healy coal bed in the Buffalo-Lake DeSmet area is the thickest known coal bed in the contiguous United States (Jones, 2010), no significant, large-scale mining has occurred in the Wasatch coal beds in the PRB. The USGS assessment of coal resources within the NWPRB assessment area (Scott and others, 2010) and the SWPRB assessment have been used to model two potentially minable coal beds, the Upper Healy and the Healy. Because these two coal beds occur within both the NWPRB and SWPRB assessment areas (fig. 1), a smaller area of assessment was created from both of the assessment areas that encompass the complete areal extent of these two coal beds.

Resources of these two coal beds that are available for mining are shown in table 10. The resources are based on coal bed thicknesses of at least $5 \mathrm{ft}$, overburden not greater than $500 \mathrm{ft}$, and a composite stripping ratio of not greater than 10:1. Figure 95 shows the extent of the two coals where the stripping ratio is up to 5:1; figure 96 shows their extent where the ratio exceeds 5:1 and up to 10:1. The figures also show the mining restrictions applicable to the cities of Sheridan and Buffalo, interstate highways 25 and 90, the Burlington Northern Santa Fe Railway route, and Lake DeSmet.

A comparison of figures 95 and 96 shows that the majority of the two coal beds have composite stripping ratios of greater than 5:1 that are less favorable from a recovery standpoint. Furthermore, figures 95 and 96 show that the restrictions for Buffalo, Lake DeSmet, and the interstate highways significantly impact the availability of the coal beds for mining, especially where the composite stripping ratios are most favorable for mining (less than 5:1). In addition, the upper portions of these coal beds have been burned over much of the area (Smith and others, 1972; Glass, 1997) and the extent of the resulting clinker can be seen in figures 95 and 96 .

It is suspected that coal quality issues might also be a contributing factor for the lack of significant mine development in the Buffalo-Lake DeSmet area. Luppens (2010) reviewed the published coal quality data from the BuffaloLake DeSmet area and significant problems were discovered from the standpoint of both representiveness of the samples analyzed and anomalous moisture values. The published and predicted coal quality data values are shown in table 5 . As discussed earlier, the USGS cores all had high ash layers greater than $3 / 8$ inch removed. The USGS cores adjusted to a more reasonable moisture content of 30.5 percent; ash of 8.8 percent; sulfur of 0.9 percent; and a calorific value of $7,512 \mathrm{Btu} / \mathrm{lb}$. The quality parameters are significantly inferior to coals currently being produced from the PRB (Luppens and others, 2008; and Scott and others, 2010) and would pose a competitive disadvantage for developing the Lake DeSmet resources.

Due to the combination of effects within this areaincluding limited favorable stripping ratios, restrictions to mining, predicted inferior coal quality, and areas of clinker-a reserve analysis was performed for neither the Upper Healy nor the Healy coal beds. Although small areas of these beds could possibly be mined in the future, this scenario was not addressed here because of the regional scope of this assessment and the lack of sufficient data necessary to outline specific small areas for potential surface mining.

\section{Underground Coal Economic Analysis Results}

The Southwestern Powder River Basin assessment area has limited shallow resources available for surface mining. Although no coal is currently being mined underground, there are significant resources in deep, thick coal beds. An assessment was performed to define the volume of these thick coal beds and define a threshold where underground mining might become a viable alternative to surface mining.

\section{Underground Coal Resources}

Eight coal beds within the SWPRB assessment area were assessed as potentially minable by underground methods. These coal beds, which have favorable thicknesses, depths, and areal extents for possible underground extraction in the future, are the Roland (Baker, 1929), Smith, Anderson, Dietz 3, Canyon, Lower Canyon, Werner, and Otter. Except for limited areas under major drainages, such as the Powder River, the depths to the Roland (Baker, 1929), Smith, Anderson, and Dietz 3 coal beds exceed 1,000 ft in depth. Essentially all of the Canyon, Lower Canyon, Werner, and Otter 
Table 10. Available resources for beds greater than or equal to 5.0 foot thick, overburden less than or equal to 500 feet, and < 10:1 strip ratio, reported in millions of short tons by stripping ratio for the Southwestern Powder River Basin assessment area. Resource includes coal plus partings. Columns may not sum exactly due to rounding.

\begin{tabular}{|c|c|c|c|c|c|c|c|c|c|c|c|c|c|}
\hline \multirow{2}{*}{$\begin{array}{l}\text { Coal } \\
\text { Bed } \\
\text { Name }\end{array}$} & \multicolumn{10}{|c|}{ Stripping Ratio } & \multirow{2}{*}{$\begin{array}{c}\text { Total } \\
\text { 1:1-5:1 }\end{array}$} & \multirow{2}{*}{$\begin{array}{c}\text { Total } \\
6: 1-10: 1\end{array}$} & \multirow{2}{*}{$\begin{array}{c}\text { Total } \\
(1: 1-10: 1)\end{array}$} \\
\hline & 1:1 & $2: 1$ & $3: 1$ & $4: 1$ & $5: 1$ & 6:1 & 7:1 & $8: 1$ & $9: 1$ & 10:1 & & & \\
\hline $\begin{array}{l}\text { Upper } \\
\text { Healy }\end{array}$ & 0 & 72 & 133 & 3 & & 585 & 555 & 6 & 267 & 4 & 75 & 7 & 3,092 \\
\hline Healy & 194 & 319 & 431 & 502 & 645 & 681 & 581 & 471 & 369 & 325 & 2,091 & 2,427 & 4,518 \\
\hline Total & 194 & 391 & 564 & 845 & 1,172 & 1,266 & 1,136 & 877 & 636 & 529 & 3,166 & 4,444 & 7,610 \\
\hline
\end{tabular}

coal beds are found at depths of $1,000 \mathrm{ft}$ or greater. Table 11 shows the coal resources associated with these eight coal beds, with the resources for each bed divided into two thickness categories $(10-20 \mathrm{ft}$ and $>20 \mathrm{ft})$ in combination with two depth categories (500-1,500 ft and 1,500-2,000 ft). Estimated optimal coal bed thicknesses for longwall system applications for the PRB are 10-20 ft. However, current longwall cutter heights are limited to a maximum of $20 \mathrm{ft}$.

As can be seen in table 11, the Smith coal bed contains the largest amount of potentially minable underground resources ( $82 \mathrm{BST}$ ); the Canyon coal bed contains the next largest amount (52 BST), followed by the Lower Canyon coal bed (24 BST). Collectively, these three coal beds constitute 158 BST or 79 percent of the total potentially underground minable resources of 201 BST. It must be emphasized that these potentially minable resource estimates represent a best case scenario, since an inventory of restrictions to underground mining has not been performed. It is certain that various restrictions-such as the geotechnical constraints of adverse roof and floor conditions, local coal bed thinning, and hydrology issues-would reduce the amount of coal resources ultimately recovered. Nevertheless, the deep, thick coal beds in the Southwestern Powder River Basin assessment area represent a significant future resource.

\section{Underground Mining Model Analysis}

Four economic mining cases were evaluated for their potential breakeven cost per ton. Though the mining height varied from 10 to $20 \mathrm{ft}$, and the depth from 1,000 to 2,000 ft, the average breakeven cost per ton was about $\$ 31.00$ at an interest rate of 8 percent for a $20 \mathrm{yr}$ project life. Major mining costs are summarized in tables 7 and 8 .

The width of the typical longwall panel in the mining model is the main parameter affecting the economic results of the four cases in table 6 . As the mining height was doubled, the longwall face width was halved, which resulted in shorter cutting time for each exposed longwall face; larger and more expensive (but fewer) roof shields; and decreased chain pillar recovery. Doubling of the mining depth affected surface and underground support systems (increased headframe structures, larger diameter hoisting ropes, additional shaft fixtures, surface ventilation fans, increased shaft depths and ramp length, and increased inclined conveyor length) and mining recovery.

The average cost of mining coal in the SWPRB assessment area using the longwall method for 10-20 ft thickness at 1,000-2,000 ft depth was about $\$ 31 /$ ton (tables 7 and 8) ( $\$ 0.75 /$ ton range from low to high values). The estimated calorific values of the deep coals range from about $9,300 \mathrm{Btu} / \mathrm{lb}$ at $1,000 \mathrm{ft}$ in depth to $10,000 \mathrm{Btu} / \mathrm{lb}$ at 2,000 ft in depth (fig. 93). Although the mining costs increase with depth, those higher costs might be offset by the realization of progressively higher quality coal with depth.

The price for Powder River Basin coal for the week ending October 25, 2010 was $\$ 14.10$ for coal at $8,800 \mathrm{Btu} / \mathrm{lb}$ and $\$ 11.60$ for 8,400 Btu/lb (Platts Coal Outlook, 2010). Because the current market price is approximately double the current market sales price, none of the coal resources in the eight deep coal beds in the SWPRB assessment area can currently be classified as reserves. However, longwall model analysis demonstrates that as the surface mining depths continue to increase in other parts of the PRB, underground exploitation of the deep, thick coal resources of the SWPRB assessment area may become a more viable alternative as long as the demand for coal remains strong.

\section{Conclusions}

The assessment of the SWPRB assessment area adds to the resource and reserve estimates in the PRB. The extensive amount of coalbed methane development in the PRB over the last ten years has provided a wealth of new, publicly available data, especially in the SWPRB assessment area. An important distinction of the SWPRB assessment area compared to the other PRB assessments (Luppens and others, 2008; Scott and others, 2010) is the absence of the extensive thick, shallow Wyodak coal zone. Some of the key results of this assessment are as follows: 
Table 11. Underground coal resources for the Southwestern Powder River Basin assessment area (millions of short tons).

\begin{tabular}{lrrrrrrrrr}
\hline \multirow{2}{*}{ Coal thickness and depth } & \multicolumn{10}{c}{ Coal Bed Name } \\
\cline { 2 - 11 } & $\begin{array}{l}\text { Roland } \\
\text { (Baker) }\end{array}$ & Smith & Anderson & Dietz 3 & Canyon & $\begin{array}{l}\text { Lower } \\
\text { Canyon }\end{array}$ & Werner & Otter & Totals \\
\hline 10-20 feet thick, 500-1,500 feet depth & 2,756 & 4,842 & 1,725 & 1,530 & 2,350 & 2,289 & 626 & 26 & $\mathbf{1 6 , 1 4 4}$ \\
10-20 feet thick, 1,500-2,000 feet depth & 151 & 2,103 & 19 & 1,042 & 1,271 & 2,482 & 1,368 & 543 & $\mathbf{8 , 9 7 9}$ \\
Subtotal 10-20 feet thick & 2,907 & 6,945 & 1,744 & 2,572 & 3,621 & 4,771 & 1,994 & 569 & $\mathbf{2 5 , 1 2 3}$ \\
\hline$>$ 20 feet thick, 500-1,500 feet depth & 1,431 & 71,115 & 4,695 & 6,773 & 26,496 & 8,208 & 308 & 1,946 & $\mathbf{1 2 0 , 9 7 2}$ \\
$>$ 20 feet thick, 1,500-2,000 feet depth & 7 & 3,842 & 34 & 938 & 21,496 & 11,367 & 1,018 & 16,053 & $\mathbf{5 4 , 7 5 5}$ \\
Subtotal $>$ 20 feet thick & 1,438 & 74,957 & 4,729 & 7,711 & 47,992 & 19,575 & 1,326 & 17,999 & $\mathbf{1 7 5 , 7 2 7}$ \\
Coal Bed Totals & $\mathbf{4 , 3 4 5}$ & $\mathbf{8 1 , 9 0 2}$ & $\mathbf{6 , 4 7 3}$ & $\mathbf{1 0 , 2 8 3}$ & $\mathbf{5 1 , 6 1 3}$ & $\mathbf{2 4 , 3 4 6}$ & $\mathbf{3 , 3 2 0}$ & $\mathbf{1 8 , 5 6 8}$ & $\mathbf{2 0 0 , 8 5 0}$ \\
\hline
\end{tabular}

- The significant increase in the number of available drill holes has increased the resource values for measured, indicated, and inferred resources in the SWPRB. Of the total original resources, for measured and indicated resources, about 65 percent are available. This increase in data also significantly improved the geological interpretation, including revision of coal bed correlations and definition of framework channels that controlled the areal extent, thickness, and depth of individual beds.

- The new geologic coal bed models not only provide a more robust assessment of recoverable coal resources but will also facilitate other resource planning, such as development of coalbed methane resources and evaluating the environmental impacts of energy-related production.

- The Wyodak coal interval that is of paramount importance in the Gillette coal field is thinning and splitting to the west and south in the SWPRB assessment area. This thinning and splitting adversely affects strip ratios and hence the economics for any particular project. Therefore, no economic modeling of Fort Union coals for surface mining was practical.

- In the Wasatch Formation, the Upper Healy and Healy coal beds have not been mined because of the extensive development of clinker and also the poor coal quality compared to deeper coal beds. Therefore, no economic modeling of the Wasatch coals for surface mining was practical.

- Eight coal beds are present at depths from 500-2,000 $\mathrm{ft}$ within the SWPRB assessment area. These eight coal beds represent a potential underground resource of 201 BST. These resources are sub-economic and are not considered as reserves or as recoverable at this point in time.
- Fifteen significant coal beds were correlated to coal beds in the Gillette coal field and the NWPRB assessment area. These correlations show the continuous geologic structure of the coal beds across the Powder River Basin.

- The original coal resource in the SWPRB assessment area for all 23 coal beds assessed, with no restrictions applied, was calculated to be 369 BST. Available coal resources, which are part of the original coal resource that is accessible for potential mine development after subtracting all restrictions, are about 341 billion short tons ( 92.4 percent of the original coal resource). All coal resources in the SWPRB assessment area are considered to be subeconomic at this point in time.

\section{Acknowledgments}

We would like to acknowledge the following people for helping with this publication. Nicholas Jones and staff of the Wyoming State Geological Survey supplied drill-hole data. Ed Heffern, of the U.S. Bureau of Land Management in Cheyenne, Wyoming, supplied the digital file that shows the extent of clinker within the southwestern Powder River assessment area. Norman Braz, Bureau of Land Management in Casper, Wyoming, supplied the digital data that show the extent of mined out areas within the southwestern Powder River assessment area. Ron Affolter, Joe East, Dick Keefer, and Margaret Ellis, all of the USGS, provided technical reviews of the manuscript. Tim Grant of the National Energy Technology Laboratory of the U.S. Department of Energy also provided a technical edit of the manuscript. 


\section{References Cited}

Association for the Advancement of Cost Engineering International (AACE) 1997, Cost Estimate Classification SystemAs applied in engineering, procurement, and construction for the process industries, AACE International Recommended Practice No. 18R-97.

American Society for Testing and Materials (ASTM International, 2008): Annual Book of ASTM Standards, V. 05.06, Chapter D 388-05, p. 250-256.

Baker, A.A., 1929, The northward extension of the Sheridan Coal Field, Big Horn and Rosebud Counties, Montana: U.S. Geological Survey Bulletin 806-B, p. 15-67.

Carter, M.D., and Gardner, N.K., 1989, An assessment of coal resources available for development: U.S. Geological Survey Open File Report 89-362, 52 p.

Coates, D.A., and Heffern, E.L., 1999, Origin and geomorphology of clinker in the Powder River Basin, Wyoming and Montana, in Miller, W.R., ed., Coal bed methane and the Tertiary geology of the Powder River Basin: Wyoming Geological Association 50 ${ }^{\text {th }}$ Annual Field Conference Guidebook, p. 211-229, 1 plate.

Culbertson, W.C., 1987, Diagrams showing proposed correlation and nomenclature of Eocene and Paleocene coal beds in the Birney 30' X 60' quadrangle, Big Horn, Rosebud, and Powder River Counties, Montana: U.S. Geological Survey Coal Investigations Map C-113, scale 1:100,000.

Culbertson, W.C., and Mapel, M.J., 1976, Coal in the Wasatch Formation, northwest part of the Powder River Basin near Sheridan, Sheridan County, Wyoming: Chapter in Wyoming Geological Association Guidebook, $28^{\text {th }}$ Annual Field Conference, Casper, Wyoming. p. 193-201.

Culberston, W.C., Kent, B.H., and, W.J., 1979, Preliminary diagrams showing correlation of coal beds in the Fort Union and Wasatch Formations across the Northern Powder River Basin, north-eastern Wyoming and southeastern Montana: U.S. Geological Survey Open-File Report 79-1201, 11 p., 2 diagrams (no scale).

De Bruin, R.H., Lyman, R.M., Jones, R.W., and Cook, L.W., 2004, Coal bed methane in Wyoming: Wyoming State Geological Survey Information Pamphlet 7, second revision, $24 \mathrm{p}$.

Department of the Treasury, 2008, IRS Publication 946: available at: http://www.irs.gov/publications/p946, accessed in June, 2010.

Eggleston, J.R., Carter, M.D., and Cobb, J.C., 1990, Coal resources available for development-A methodology and pilot study: U.S. Geological Survey Circular 1055, 15 p.
Ellis, M.S., Molnia, C.L, Osmonson, L.M., Ochs, A.M., Rohrbacher, T.J., Mercier, T., and Roberts, N.R., 2002, Evaluation of economically extractable coal resources in the Gillette coal field, Powder River Basin, Wyoming: U.S. Geological Survey Open-File Report 02-180, 48p.

Environmental Systems Research International (ESRI), Incorporated, 2000, ArcView software version 3.2a, Environmental Systems Research International, Incorporated, $380 \mathrm{New}$ York Street, Redlands, CA 92373, USA.

Environmental Systems Research International (ESRI), Incorporated, 2001, ArcView Spatial Analyst Extension software version 2.0, Environmental Systems Research International, Incorporated, 380 New York Street, Redlands, CA 92373, USA.

Environmental Systems Research International (ESRI), Incorporated, 2006, ArcGIS software version 9.2, Environmental Systems Research International, Incorporated, $380 \mathrm{New}$ York Street, Redlands, CA 92373, USA.

Flores, R.M., Ochs, A.M., Bader, L.R., Johnson, R.C., and Vogler, D., 1999, Framework geology of the Fort Union coal in the Powder River Basin, Chapter PF in 1999 Resource Assessment of selected Tertiary coal beds and zones in the Northern Rocky Mountains and Great Plains Region: U.S. Geological Survey Professional Paper 1625-A, 2 CD-ROMs.

Glass, G.B., 1975, analyses and measured sections of 54 Wyoming coal samples: The Geological Survey of Wyoming, RI No. 11,219 p.

Glass, G. B., 1976, Update on the Powder River Coal Basin: Chapter in Wyoming Geological Association Guidebook, $28^{\text {th }}$ Annual Field Conference, Casper, Wyoming. p. 209-220.

Glass, G.B., 1980, Coal resources of the Powder River Coal Basin in Guidebook to the coal geology of the Powder River Coal Basin, Wyoming, Field trip no. 5: American Association of Petroleum Geologists Energy Minerals Division, June 12-14, 1980, p. 91-124.

Glass, G.B., and Jones, R.J., 1992, Coal Fields and Coal Beds of Wyoming: Wyoming Geological Association Reprint No. 47, p. 133-167.

Glass, G.B., 1997, Coal geology of Wyoming: Wyoming State Geological Survey Reprint No. 63, 21 p.

Glass, G.B., 1997, Coal geology of Wyoming in Sandra, A.P., ed., 1997 Keystone Coal Industry Manual, Intertec Publishing, Chicago, Illinois.

Golden Software, Inc. Surfer8 software, 2002, Golden Software, Inc., $80914^{\text {th }}$ Street, Golden, Colorado, 80401-1866, USA. 
Golightly, D.W. and Simon, F.O., 1989, Methods for sampling and inorganic analysis of coal: U.S. Geological Survey Bulletin 1823, 72p., http://pubs.usgs.gov/bul/b1823/01.htm, accessed September, 2010.

Gallegos Research Group (GRG) Corporation, StratiFact software version 4.57, 1998, GRG Corporation, 4175 Harlan Street, Wheatridge, Colorado, 80033-5150, USA.

Jones, N.R., 2010, Coal geology of Wyoming in Johnson, P., publisher., 2010 Keystone Coal Industry Manual, Mining Media International, Jacksonville, Florida.

Kent, B.H., Berlage, L.J., and Boucher, E.M., 1980, Stratigraphic framework of coal beds underlying the western part of the Recluse 1 x 1/2-degree quadrangle, Campbell County, Wyoming: U.S. Geological Survey Coal Investigations Map C-81C, 2 plates, scale, 1:100,000.

Law, B.E., Barnum, B.E., and Wollenzien, T.P., 1979, Coal bed correlations in the Tongue River Member of the Fort Union Formation, Monarch, Wyoming, and Decker, Montana, areas: U.S. Geological Survey Miscellaneous Investigations Series Map I-1128, scale, 1:24,000.

Luppens, J.A., 2011, A critical review of published coal quality data from the southwestern part of the Powder River Basin, Wyoming: U.S. Geological Survey, Open File Report 2011-1148, 40 p.

Luppens, J.A., Scott, D.C., Haacke, J.E., Osmonson, L.M., Rohrbacher, T.J., and Ellis, M.S., 2008, Assessment of Coal Geology, Resources, and Reserves in the Gillette coal field, Powder River Basin, Wyoming: U.S. Geological Survey Open File Report 2008-1202, 127 p.

Luppens, J.A., Rohrbacher, T.J., Osmonson, L.M., and Carter, M.D., 2009, Coal resource availability, recoverability, and economic evaluations in the United States - A Summary, in Pierce, B.S., and Dennen, K.O., eds., The National Coal Resource Assessment Overview: U.S. Geological Survey Professional Paper 1625-F, Chapter D, 17 p.

Mapel, W.J., Schopf, J.M., and Gill, J.R., 1953, A thick coal bed near Lake DeSmet, Johnson County, Wyoming: U.S. Geological Survey Circular 228, 47 p.

Mapel, W.J., 1954, Geology and coal resources of the Lake DeSmet Area, Johnson County, Wyoming: U.S. Geological Survey Coal Investigations Map C23, scale, 1:50,000.

Mapel, W.J., 1959, Geology and coal resources of the BuffaloLake DeSmet Area Johnson and Sheridan Counties Wyoming: U.S. Geological Survey Bulletin 1078, 148 p.

Mentor Consultants, 2005, PC/Cores, Post Office Box 3147, Glen Ellyn, Illinois, USA, 60137.
Molnia, C.L., and Pierce, F.W., 1992, Cross sections showing coal stratigraphy of the southwestern Wyoming Powder River Basin, Wyoming and Montana: U.S. Geological Survey Miscellaneous Investigations Series Map I-1959-D, scale 1:500,000.

Molnia, C.L., Osmonson, L.M., Wilde, E.M., Biewick, L.R.H., Rohrbacher, T.J., and Carter, M.D., 1999, Coal availability and recoverability studies in the Powder River Basin, Wyoming and Montana, in Fort Union Coal Assessment Team, 1999 resource assessment of selected Tertiary coal beds and zones in the Northern Rocky Mountains and Great Plains Region: U.S. Geological Survey Professional Paper 1625-A, 119 p.

Obernyer, S.L., 1978, Basin-margin depositional environments of the Wasatch Formation in the Buffalo-Lake DeSmet area, Johnson County, Wyoming, in Proceedings of the second symposium on the geology of Rocky Mountain coal-1977: Colorado Geological Survey Resource Series 4, p. 49-65.

Obernyer, S.L., 1980, The Lake DeSmet coal seam, in Guidebook to the coal geology of the Powder River Coal Basin, Wyoming: Geological Survey of Wyoming Public Information Circular 14, p. 31-70.

Office of the Federal Register, 2003, code of federal regulations, title 43, part 3460, subpart 3461.5: National Archives and Records Administration, p. 588-593, http://www. gpoaccess.gov/cfr/retrieve.html, accessed June, 2009.

Olive, W.W., 1957, The Spotted Horse Coal Field, Sheridan and Campbell Counties, Wyoming: U. S. Geological Survey Bulletin 1050, 83 p.

PACIFICORP, 2008, Dave Johnston Power Plant 50 $0^{\text {th }}$ anniversary and Glenrock Wind Farm community celebration, PACIFICOR, Portland, OR.

Pierce, B.S., and Dennen, K.O., eds., 2009, The National Coal Resource Assessment Overview: U.S. Geological Survey Professional Paper 1625-F, 402 p.

Platts, 2010, Coal Outlook: Platts, a division of The McGrawHill Companies, New York, NY, Vol. 34, No. 37, September 13, 2010, $12 \mathrm{p}$.

Public Law 95-87, 1977 Surface Mining Control and Reclamation Act of 1977, (30 U.S.C. 1201 et seq.), 115 p. http:// www.osmre.gov/smcra.htm, accessed June 2009.

Rice, C.A., Ellis, M.S., and Bullock, J.H., Jr., 2000, Water coproduced with coal bed methane in the Powder River Basin, Wyoming-preliminary compositional data: U.S. Geological Survey Open-File Report 00-372, 20 p. 
Scott, D.C., Haacke, J.E., Osmonson L.M., Luppens, J.A., Pierce, P.E., and Rohrbacher, T.J., 2010, Assessment of Coal Geology, Resources, and Reserves in the Northern Wyoming Powder River Basin, Wyoming: U.S. Geological Survey Open-File Report OF-2010-1294, 137 p.

Smith, J.B., Ayler, M.F., Knox, C.C., and Pollard, B.C., 1972, Strippable coal reserves of Wyoming: U.S. Bureau of Mines Information Circular IC 8538, 51 p.

Supreme Court of the United States, 1999, Amoco Production Co. v. Southern Ute Tribe (98-830), 526, U.S. 865, available at http://www.law.cornell.edu., accessed June 2010.

Stermole, F.J., 1974, Economic evaluation and investment decision methods; second edition: Investment Evaluations Corporation, 2000 Goldenvue Dr., Golden, Colorado, 80401, p. 14.

Stricker, G.D., Flores, R.M., Trippi M.H., Ellis, M.S., Olson, C.M., Sullivan, J.E., and Takahashi, K.I., 2007, Coal quality and major, minor, and trace elements in the Powder River, Green River, and Williston Basins, Wyoming and North Dakota: U.S. Geological Survey Open-File Report 20071116, $31 \mathrm{p}$.

Taff, J.A., 1909, The Sheridan coal field, Wyoming, in Coal and lignite, Pt. 2: U.S. Geological Survey Bulletin 341, p. 123-150.

Testa, S.M., and T.J. Pratt, 2003, Sample preparation for coal and shale gas resource assessment; in Proceedings, International Coalbed Methane Symposium, Tuscaloosa, Alabama, May 5-9, 2003: University of Alabama, Paper 356, 12 p.

Thomas, Larry, 2002, Coal Geology: Dargo Associates Ltd., John Wiley and Sons, LTD., West Sussex, England, 365 p.

U.S. Bureau of Land Management, 1999, Final Environmental Impact Assessment WY-070-EA-9-191 for the proposed Enron (Belco) - BLM coal lease exchange, Casper, Wyoming Field Office, 187 p.

U.S. Bureau of Land Management, 2001, Buffalo resource management plan, Appendix D-coal screening process: available at: http://www.blm.gov/rmp/WY, accessed June 2009.

U.S. Bureau of Land Management, 2003, Final Environmental Impact Statement and Proposed Plan Amendment for the Powder River Basin Oil and Gas Project (WY-070-02-065) Buffalo, Wyoming Field Office, 397 p.

U.S. Energy Information Administration, 2010, Energy glossary: http://www.eia.doe.gov/glossary/index.cfm, accessed September, 2010.
U.S. Environmental Protection Agency, 1980, Standards of performance for new stationary source a compilation as of July 1, 1980; U.S. Environmental Protection Agency, Office of Enforcement, Office of General Enforcement, Division of Stationary Source Enforcement. Washington. D.C., 868 p., http://nepis.epa.gov/Exe/ZyNET.exe/2000WROU.txt., accessed September, 2010.

U.S. Environmental Protection Agency, 2005, AirControl NET Documentation report; Pechan Report No. 05.09.009/9010.463, H. E, Pechan \& Associates, Springfield, VA, 1685 p. http://www.epa.gov/air/ozonepollution/ SIPToolkit/documents/DocumentationReport.pdf., accessed September, 2010.

Supreme Court of the United States, 1999, Amoco Production Co. v. Southern Ute Tribe (98-830), 526, U.S. 865.

Wegemann, C.H., 1912, The Sussex coal field, Johnson, Natrona, and Converse Counties, Wyoming: U.S. Geological Survey Bulletin 471, p. 441-471.

Western Fuels Association, Inc., 1983, Final Showing Report Stevens Properties Prospecting Permits, Converse County, Wyoming: Western Fuels Association, Inc., Denver, Colorado, $25 \mathrm{p}$.

Wood, G.H., Jr., Kehn, T.M., Carter, M.D., and Culbertson, W.C., 1983, Coal resource classification system of the U.S. Geological Survey: U.S. Geological Survey Circular 891, $65 \mathrm{p}$.

Wyoming Oil and Gas Conservation Commission (WOGCC), 2009: available at: http://wogcc.state.wy.us, accessed June 2009. 


\section{Glossary}

The present study includes determinations of original, available, recoverable, and economically recoverable (reserves) resources. This terminology has been used in many USGS coal studies (see Wood and others, 1983; Carter and Gardner, 1989; Eggleston and others, 1990; and Molnia and others, 1999). The following definitions were applied in this resource evaluation:

- Available coal resource is the amount of the original resource that is accessible for mine development under current regulatory and land-use constraints. This resource is the original coal minus previously mined coal and coal that cannot be mined owing to land use and technical restrictions.

- Clinker is rock that is produced where coal beds burn naturally beneath the ground (Coates and Heffern, 1999).

- Coal reserves are virgin or accessed parts of a coal reserve base which could be economically extracted or produced at the time of determination considering environmental, legal, and technologic constraints. The term reserves need not signify that extraction facilities are in place or operative. Reserves include only recoverable coal; thus, terms such as "extractable reserves" are redundant and are not a part of the classification system. Reserves can be categorized as "measured" and "indicated," as underground or surface minable, by thickness of overburden, by thickness of coal in the bed, and by various quality factors (Wood and others, 1983).

- Coal resources are naturally occurring concentrations or deposits of coal in the Earth's crust, in such forms and amounts that economic extraction is currently or potentially feasible (Wood and others, 1983).

- Deterministic is having a predictable outcome due to known causes or equivalence to a previous event (AACE, 1997, p. 3).

- Discounted cash flow is the stream of the net after-tax cash flows where the cash outlays include all operating costs, taxes, and investment costs, and where revenues include cash payments of product sales.

- Hypothetical resources are a class of undiscovered resources that are either similar to known coal deposits which may be reasonably expected to exist in the same coal field or region under analogous geologic conditions or are an extension from inferred resources (Wood and others, 1983).

- Headgate is the primary roadway access and egress through which equipment, personnel, and supplies are provided to a longwall face, through which coal is con- veyed to the surface, and through which fresh air flows (Stermole, 1974, p. 14).

- Identified resources are resources whose locations, rank, quality, and quantity are known or estimated from specific geologic evidence. Identified coal resources include economic, marginally economic, and subeconomic components. To reflect varying distances from points of control or reliability, these subdivisions can be divided into demonstrated and inferred, or preferably into measured, indicated, and inferred (Wood and others, 1983).

- Interburden is noncoal material that lies between two coal beds.

- Original coal resource is the total amount of coal inplace before production. Where mining has occurred, the total of original resources is the sum of the identified resources, undiscovered resources, coal produced, and coal lost in mining (Wood and others, 1983).

- Overburden is rock including coal or unconsolidated material that overlies a specified coal bed. Overburden is reported in feet or meters and used to classify the depth to an underlying coal bed (Wood and others, 1983).

- Parting is a thin layer of stratum or noncoaly material within a coal bed which does not exceed the thickness of coal in either the directly underlying or overlying benches (Wood and others, 1983).

- Previously mined coal is coal that has already been extracted.

- Recoverable coal resource is coal that is or can be extracted from a coal bed during mining. The term "recoverable" should be used in combination with "resources" and not with "reserves" (Wood and others, 1983).

- Reliability categories are based on the distance from points of measurement or sampling. The measured, indicated, inferred, and hypothetical resource categories, as defined, indicate the relative reliability of tonnage estimates as related to distance from points of thickness control of particular parts of a coal deposit. The reliability categories are not indicative of the reliability of the basic data (that is, the accuracy of coal measurements, or the accuracy of location of the coal outcrop. It is assumed that all basic data used in resource estimation have been judged reliable by the estimator and that unreliable data have been discarded (Wood and others, 1983).

- Measured-Tonnage estimates computed by extrapolation of thicknesses of coal for a radius of $0.25 \mathrm{mi}(0.4 \mathrm{~km})$ from a point of measurement. 
- Indicated-Tonnage estimates computed by extrapolation of thicknesses of coal for a radius of 0.25 to $0.75 \mathrm{mi}(0.4$ to 1.2 kilometers $(\mathrm{km}))$ from a point of measurement.

- Inferred-Tonnage estimates computed by extrapolation of thicknesses of coal for a radius of 0.75 to $3.0 \mathrm{mi}$ ( 1.2 to $4.8 \mathrm{~km}$ ) from a point of measurement.

- Hypothetical-Tonnage estimates computed by extrapolation of thicknesses of coal for a radius beyond $3.0 \mathrm{mi}(4.8 \mathrm{~km})$ from a point of measurement.

- Remaining resources are the original resource minus the volume of coal that had been previously mined.

- Restrictions to mining include land use, technical limitations, and unsuitability that would prohibit mining:

- Land use restrictions are constraints placed upon mining by societal policies to protect those surface features or entities that could be harmed by mining. Since laws and regulations can be modified or repealed, the restrictions, including industrial and environmental restrictions, may change. Land use restrictions include railroads, cities and towns, airports, and interstate highways.

- Technical restrictions are constraints, relating to economics and safety, placed upon mining by the state of technology or prescribed by law. These restrictions can change with advances in science and technology, or modifications in the law. In this report, geologic factors are included as technologic restrictions. Technical restrictions include coal between $2.5 \mathrm{ft}$ and $5.0 \mathrm{ft}$ thick and clinker areas.

- Unsuitability criteria are constraints used to determine if an area can be mined by surface mining methods. These include, but are not limited to, Federal land systems, dwellings, and alluvial valley floors.

- Single payment Present Worth is the equivalent present value of an expenditure or investment that is made at some future date and valuated by compounding interest (Stermole, 1974, p. 14).

- Stochastic is random variables having a probability distribution or random variables determined through conjecture (AACE, 1997, p. 3)

- Surface (strip) mining is the practice of mining a seam of mineral by first removing a long strip of overlying soil and rock. Surface mining is practical only when the ore body to be excavated is relatively near the surface. When coal seams are near the surface, it may be economical to extract the coal using open cut (also referred to as open cast, open pit, or strip) mining methods. Open cast coal mining recovers a greater proportion of the coal deposit than underground methods, as more of the coal seams may be recovered.

- Stripping (mining) ratio is the most influential economic factor in the evaluation and planning of open pit coal mines. It represents the ratio of the volume of overburden or interburden (waste) that must be removed to gain access to a unit amount of coal. For this assessment, the ratio is expressed as cubic yards of overburden to tons of coal. The stripping ratio can be approximated by dividing the total thickness of waste by the total thickness of coal. For example, given two coal beds each $5.0 \mathrm{ft}$ thick at 50 and $105 \mathrm{ft}$ in depth, the total waste and coal thicknesses would be 100 and $10 \mathrm{ft}$ respectively. A simple ratio would be $10: 1$, but a stripping ratio would be 9.1:1 (in cubic yards to a ton of coal).

- Tailgate is the secondary roadway access or egress from a longwall face and through which return air flows (SME, 1992, SME Mining Engineering Handbook, volume 1).

- Uniform Series Present Worth is the equivalent present value of multiple, equal amount payments made at regular spaced, future intervals and valuated by compounding interest (Stermole, 1974, p. 14). 


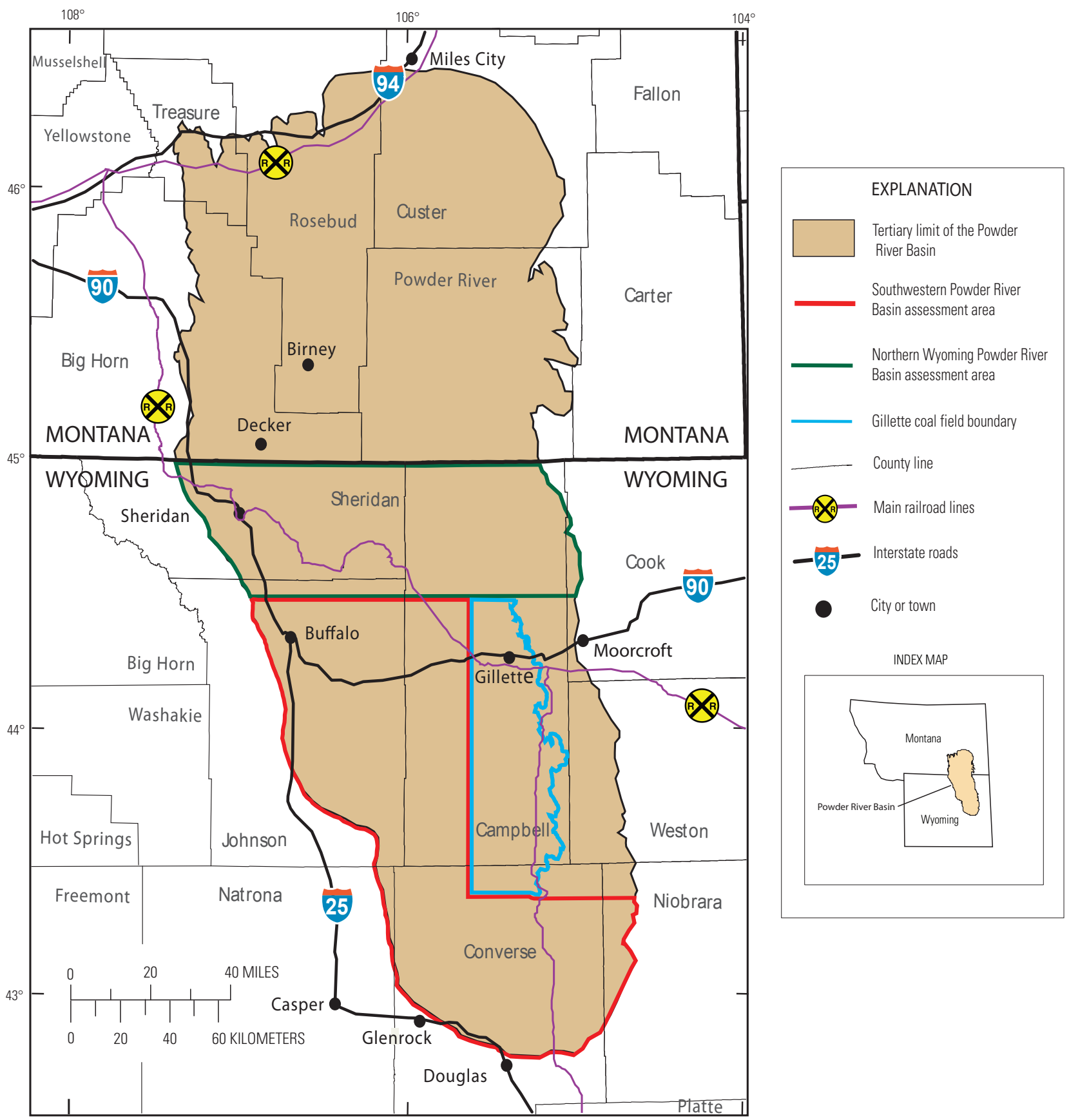

Figure 1. Location of Southwestern Powder River Basin assessment area, Northern Wyoming Powder River Basin assessment area (Scott and others, 2010), and Gillette coal field, (Luppens and others, 2008) Wyoming and Montana. 


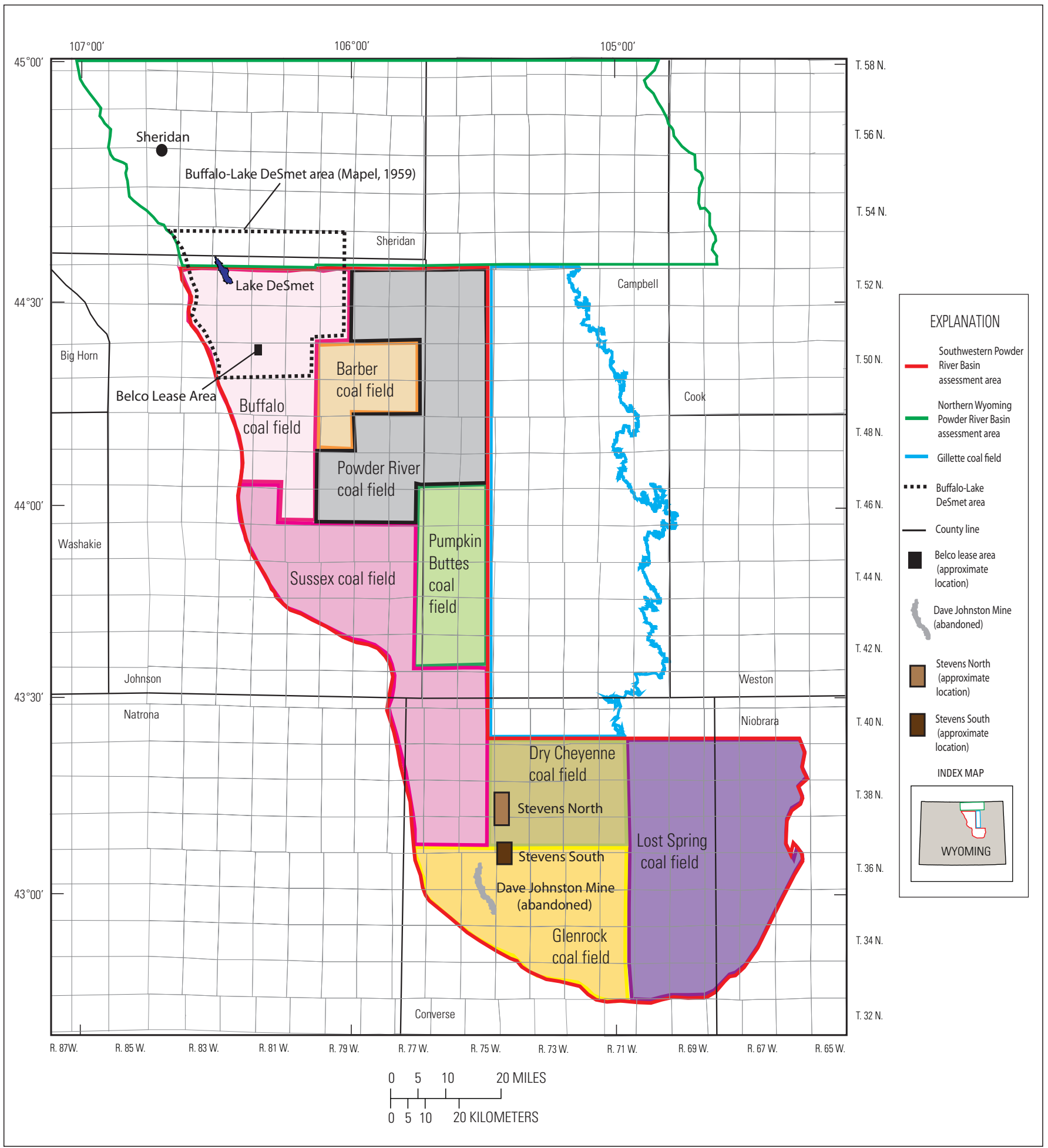

Figure 2. Coal fields in the Southwestern Powder River Basin assessment area (modified from Glass, 1976). 


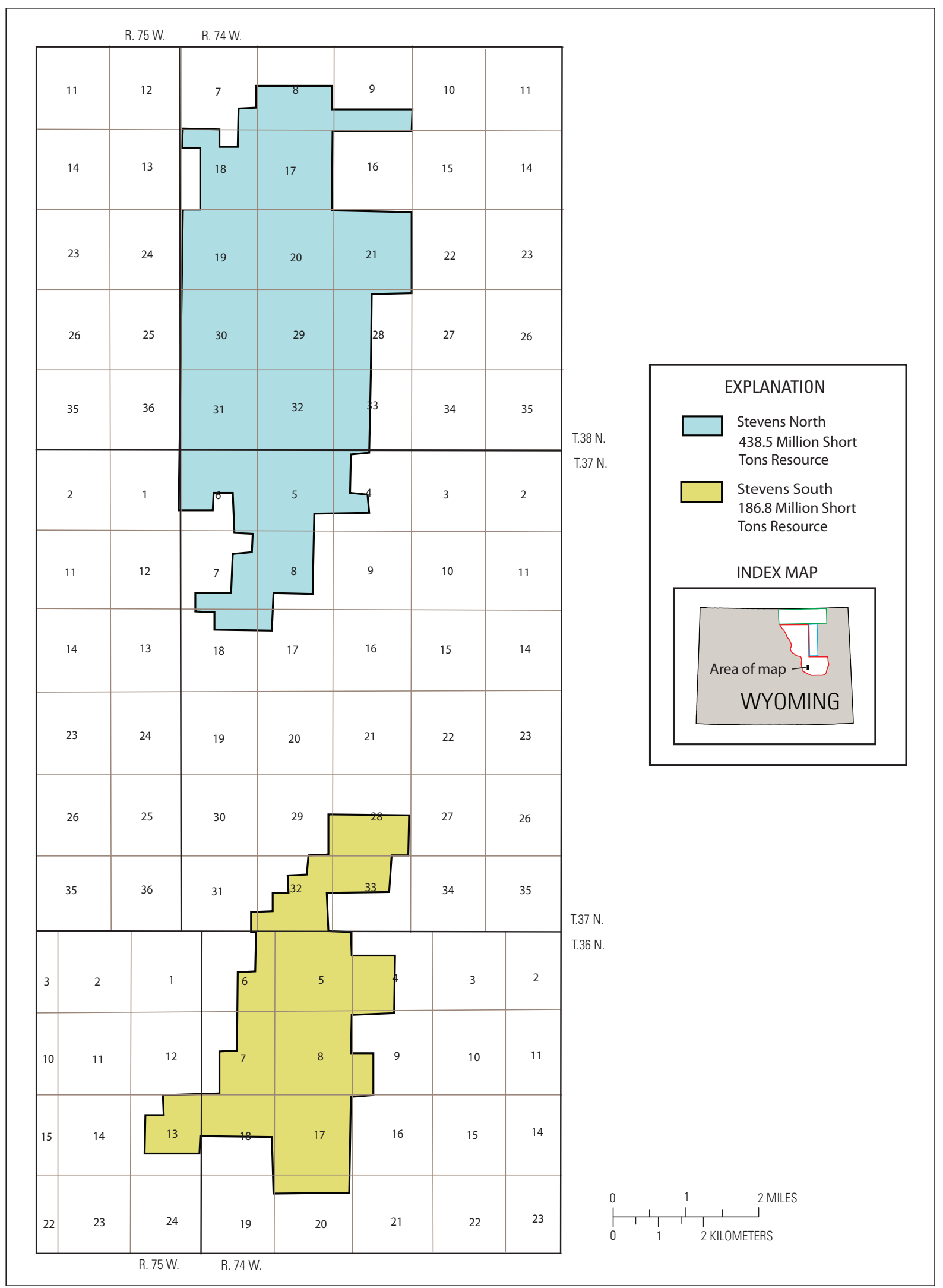

Figure 3. Areas of coal resources in the Stevens Preference Right Lease Applications (PRLA's) in the Southwestern Powder River Basin assessment area. 


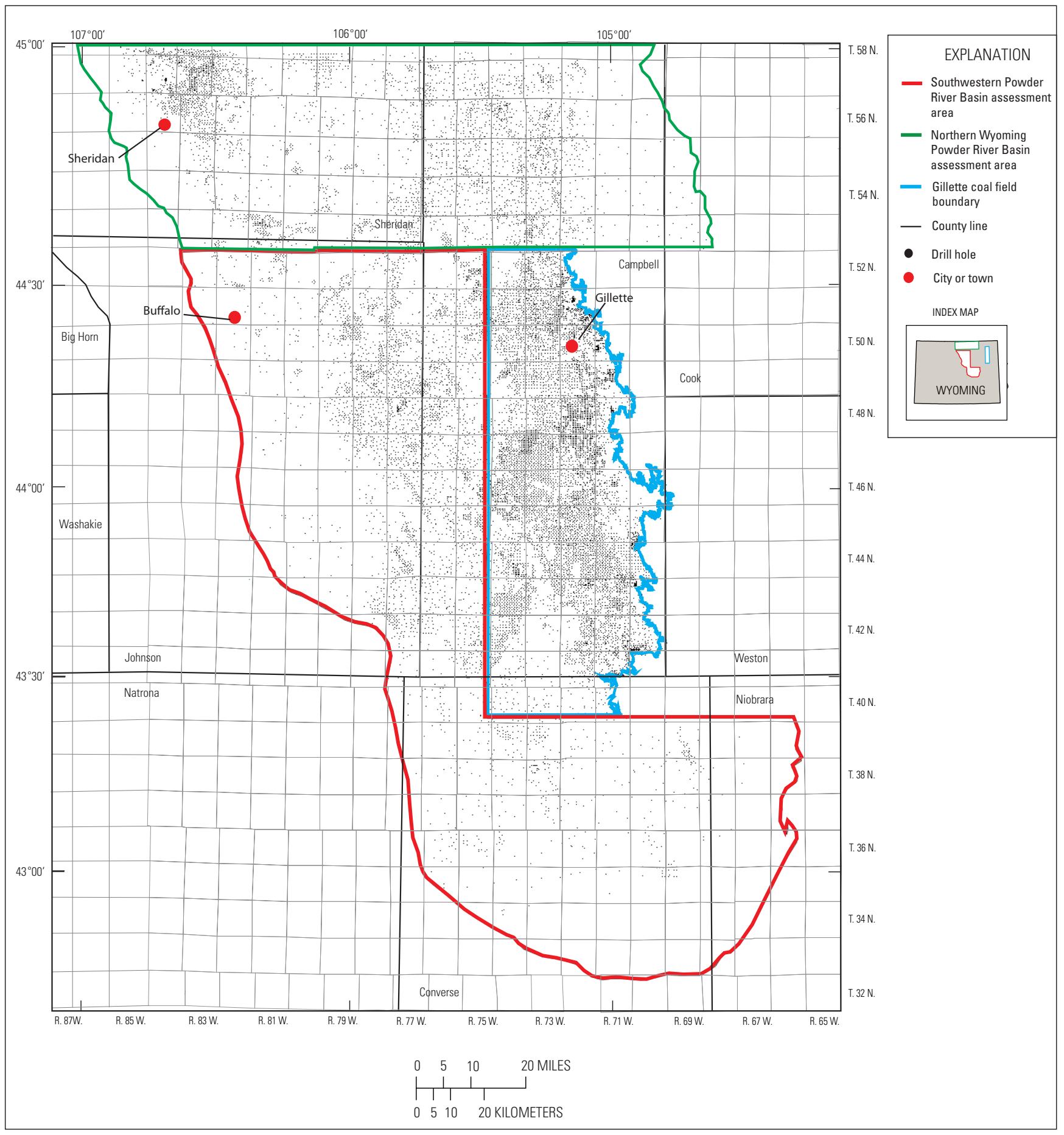

Figure 4. Drill hole locations in the Powder River Basin, Wyoming. 


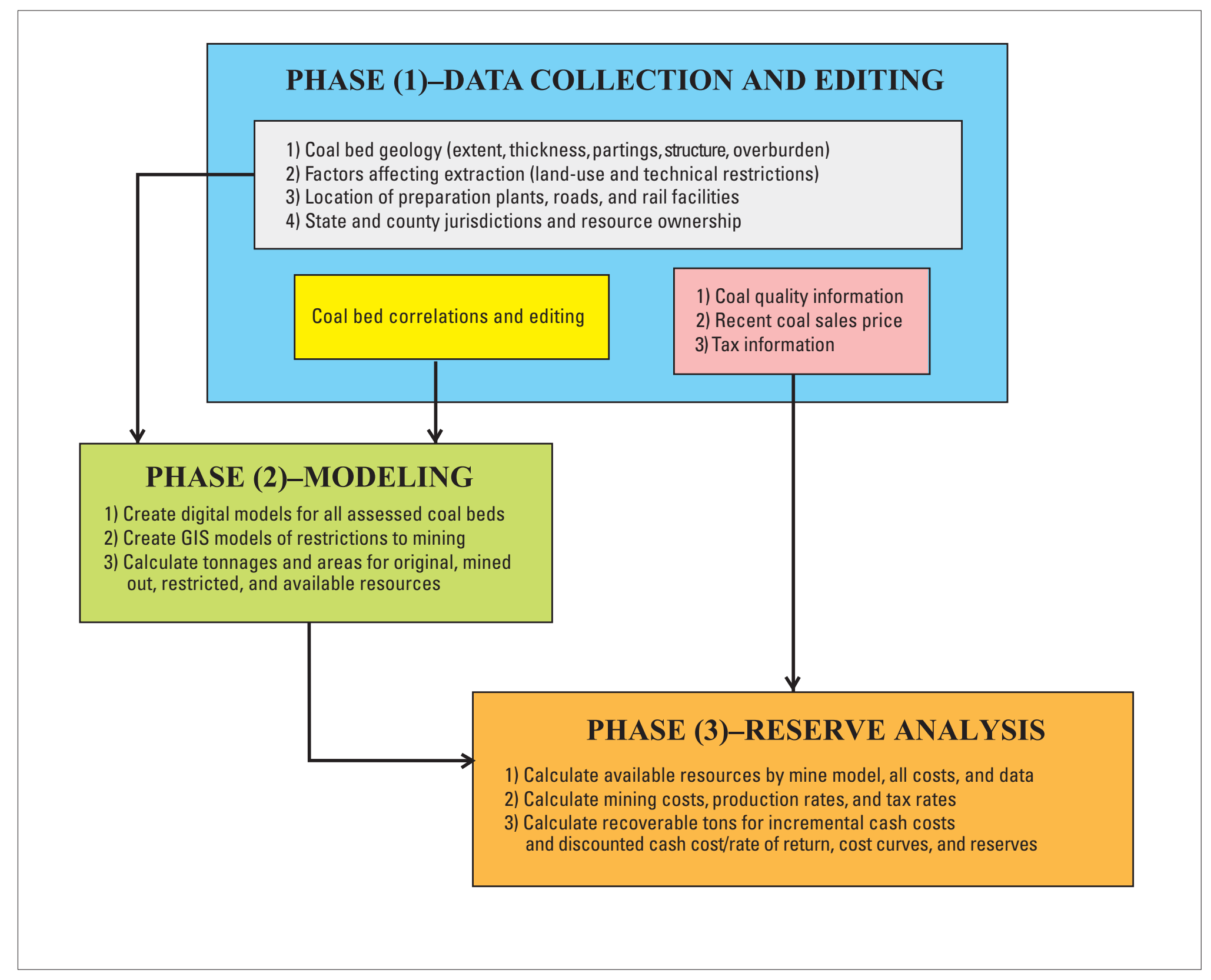

Figure 5. Flow chart showing generalized methodology used during coal resource and reserve evaluation. 


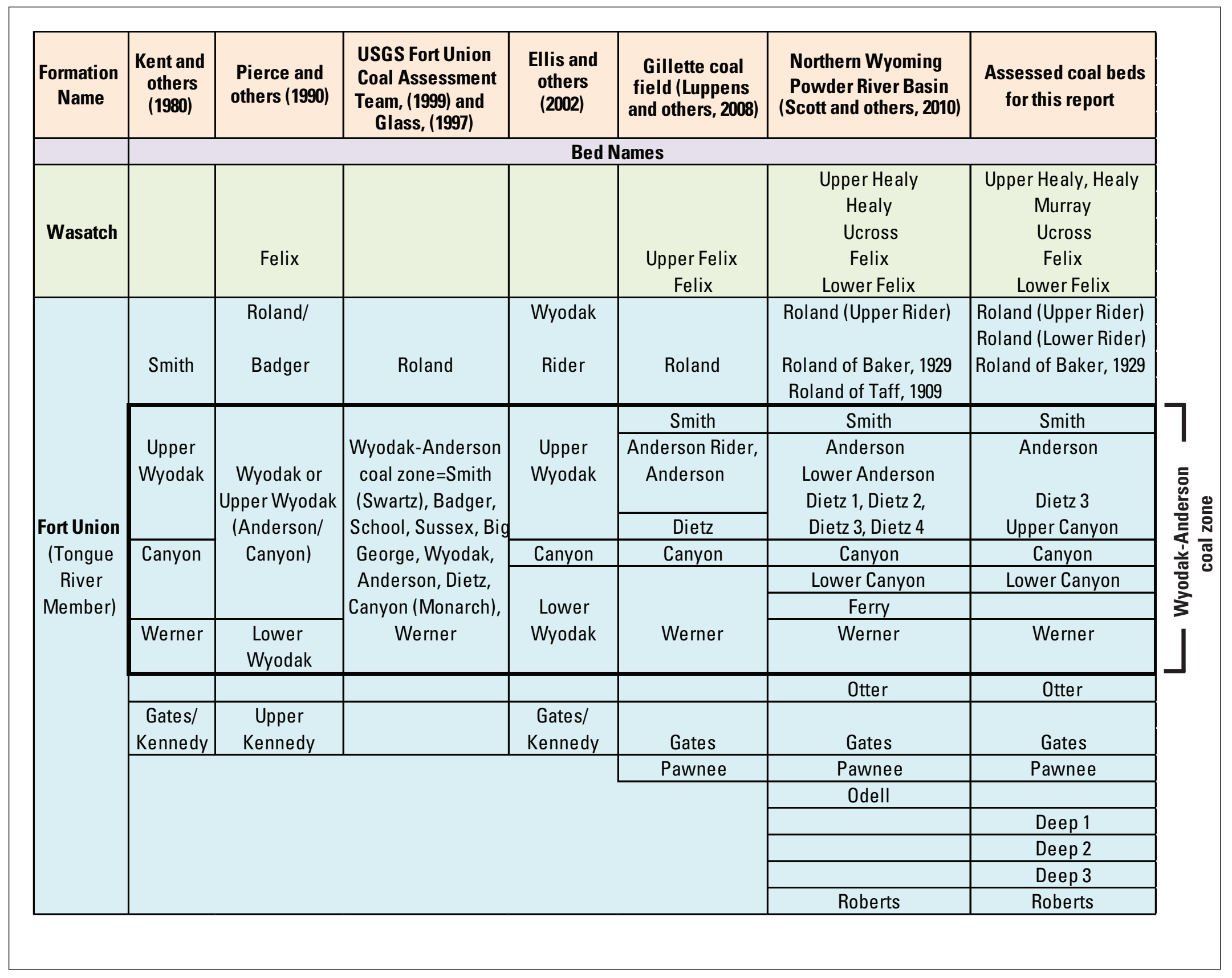

Figure 6. Coal bed and coal zone names used in different publications in the Powder River Basin. 


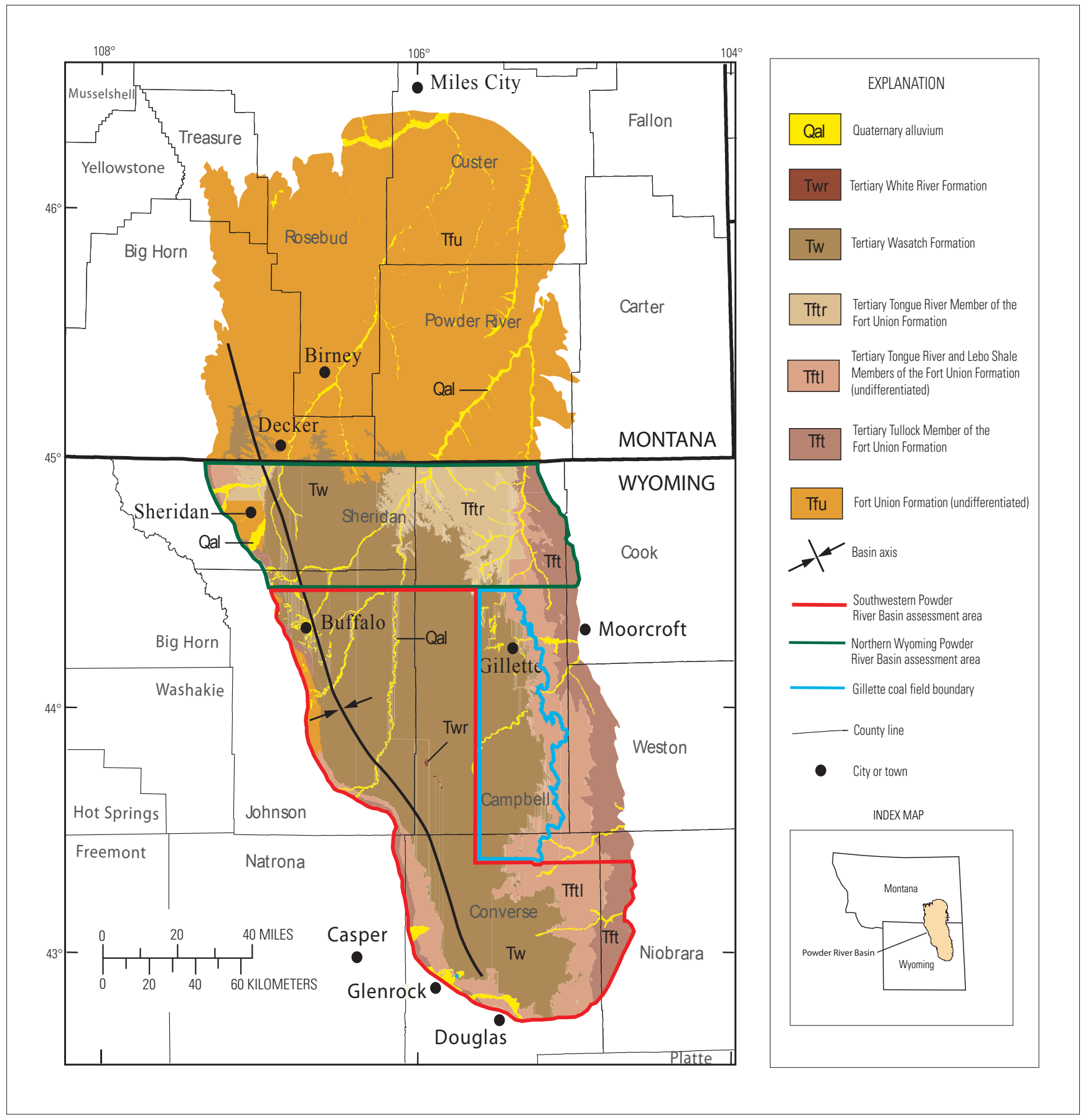

Figure 7. Generalized geology of the Powder River Basin, Wyoming and Montana (modifed from Flores and others, 1999). 


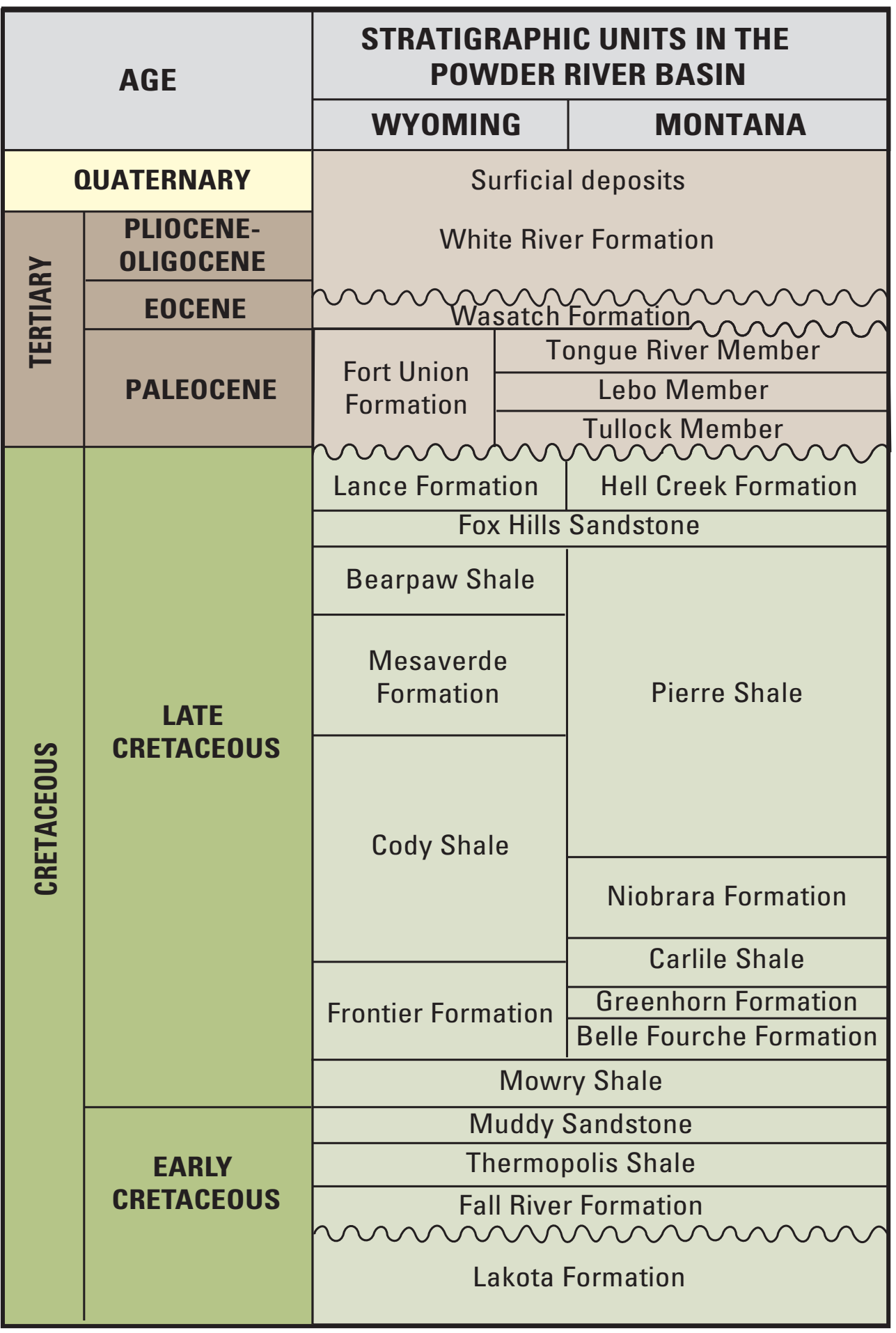

Figure 8. Generalized stratigraphic column for the Powder River Basin, Wyoming and Montana (modified from Ellis and others, 2002). 


\begin{tabular}{|c|c|c|c|c|}
\hline \multicolumn{5}{|c|}{ Coal Stratigraphy } \\
\hline $\begin{array}{l}\text { Formation } \\
\text { name }\end{array}$ & $\begin{array}{c}\text { Coal Beds } \\
\text { identified } \\
\text { in this assessment }\end{array}$ & $\begin{array}{l}\text { Coal Bed Names/ } \\
\text { Resources } \\
\text { calculated } \\
\text { (see tables } 1,2,3)\end{array}$ & $\begin{array}{c}\text { Maximum } \\
\text { thickness (ft) }\end{array}$ & $\begin{array}{c}\text { Average } \\
\text { thickness (ft) }\end{array}$ \\
\hline \multirow{7}{*}{ Wasatch } & Upper Healy & Upper Healy & 66 & 10 \\
\hline & Healy & Healy & 75 & 13 \\
\hline & Murray & Murray & 14 & 3 \\
\hline & \begin{tabular}{|l|} 
Ucross \\
\end{tabular} & Ucross & 38 & 9 \\
\hline & Upper Felix & & & \\
\hline & Felix & Felix & 48 & 7 \\
\hline & Lower Felix & Lower Felix & 35 & 7 \\
\hline \multirow{30}{*}{$\begin{array}{c}\text { Fort Union } \\
\text { (Tongue River } \\
\text { Member) }\end{array}$} & Roland (Upper Rider) & Roland (Upper Rider) & 32 & 4 \\
\hline & Roland (Lower Rider) & Roland (Lower Rider) & 19 & 4 \\
\hline & Roland of Baker (1929) & Roland of Baker (1929) & 36 & 7 \\
\hline & Roland of Taff (1909) & & & \\
\hline & Smith Rider & & & \\
\hline & Smith & Smith & 266 & 32 \\
\hline & Anderson (Upper Rider) & & & \\
\hline & Anderson (Lower Rider) & & & \\
\hline & Anderson & Anderson & 61 & 10 \\
\hline & Lower Anderson & & & \\
\hline & \begin{tabular}{|l|} 
Dietz 1 \\
\end{tabular} & & & \\
\hline & Dietz 2 & & & \\
\hline & Dietz 3 & Dietz 3 & 119 & 14 \\
\hline & Dietz 4 & & & \\
\hline & Upper Canyon & Upper Canyon & 31 & 8 \\
\hline & Canyon & Canyon & 182 & 29 \\
\hline & Lower Canyon & Lower Canyon & 81 & 16 \\
\hline & Ferry Rider & & & \\
\hline & Ferry & & & \\
\hline & Werner & Werner & 76 & 8 \\
\hline & \begin{tabular}{|l|} 
Otter Rider \\
\end{tabular} & & & \\
\hline & 0 Otter & Otter & 158 & 24 \\
\hline & Gates & Gates & 122 & 13 \\
\hline & Pawnee & Pawnee & 19 & 5 \\
\hline & \begin{tabular}{|l|l|} 
Odell \\
\end{tabular} & & & \\
\hline & Deep 1 & Deep 1 & 32 & 9 \\
\hline & Deep 2 & Deep 2 & 29 & 5 \\
\hline & Deep 3 & Deep 3 & 32 & 8 \\
\hline & Deep 4 & & & \\
\hline & Roberts & Roberts & 36 & 9 \\
\hline
\end{tabular}

Figure 9. Stratigraphy of coal beds in the Southwestern Powder River Basin assessment area showing names used in this report (maximum and average thickness only shown for coal beds assessed for resources in this report). 


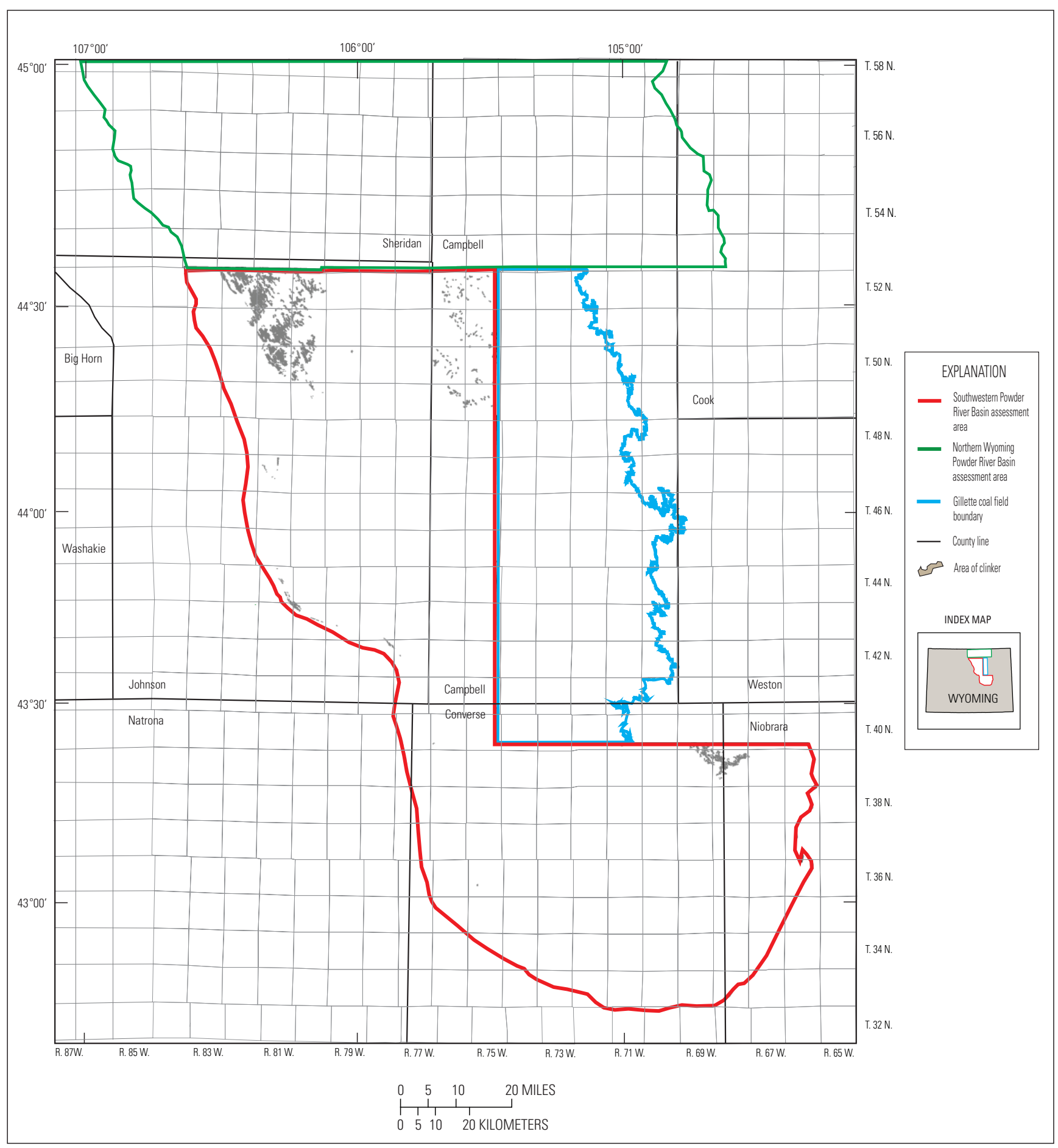

Figure 10. Location of clinker in the Southwestern Powder River Basin assessment area (modified from Heffern, 2010). 


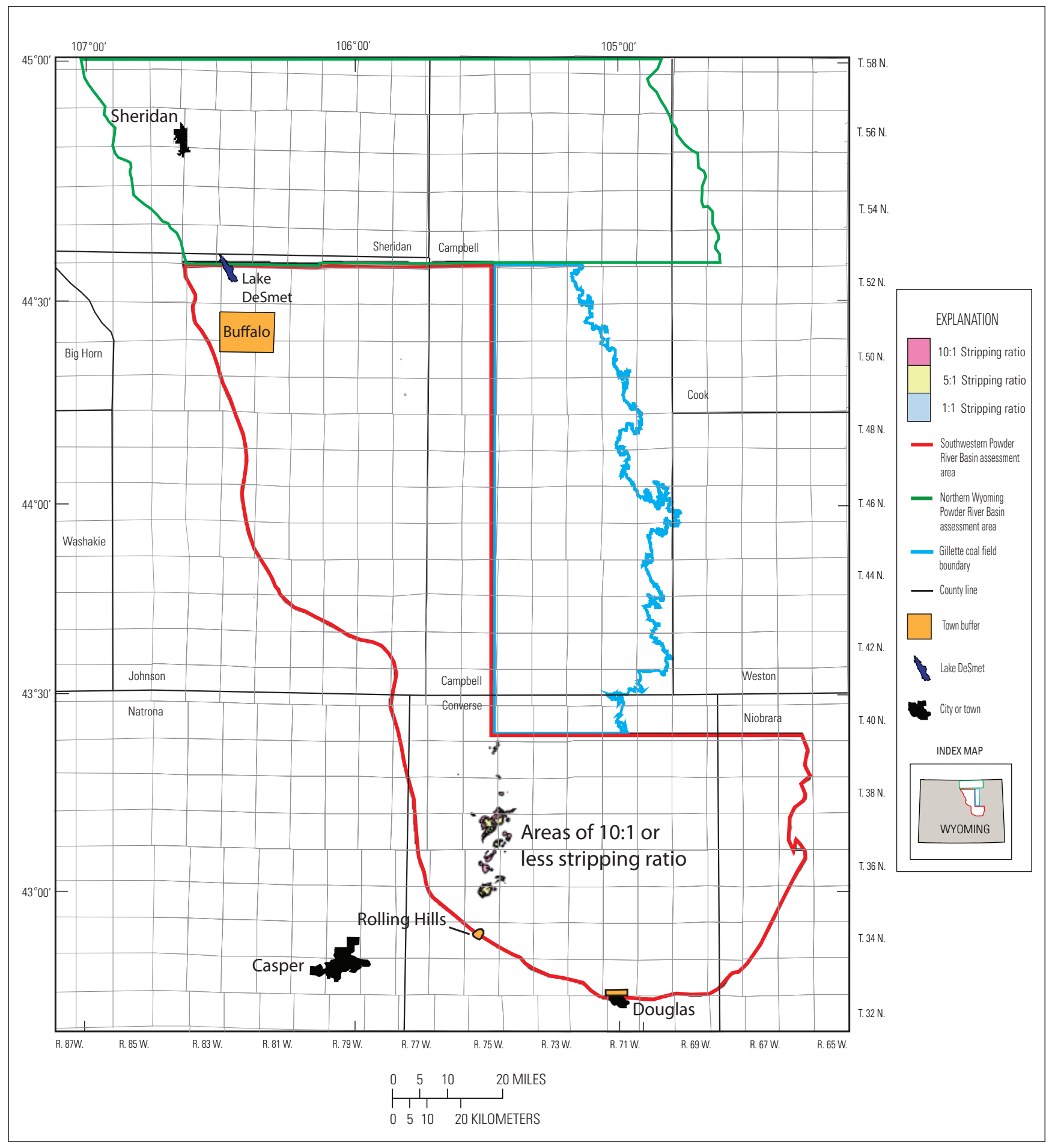

Figure 11. Stripping ratios for the Roland Baker (1929) and Smith coal beds in the Southwestern Powder River Basin assessment area. 


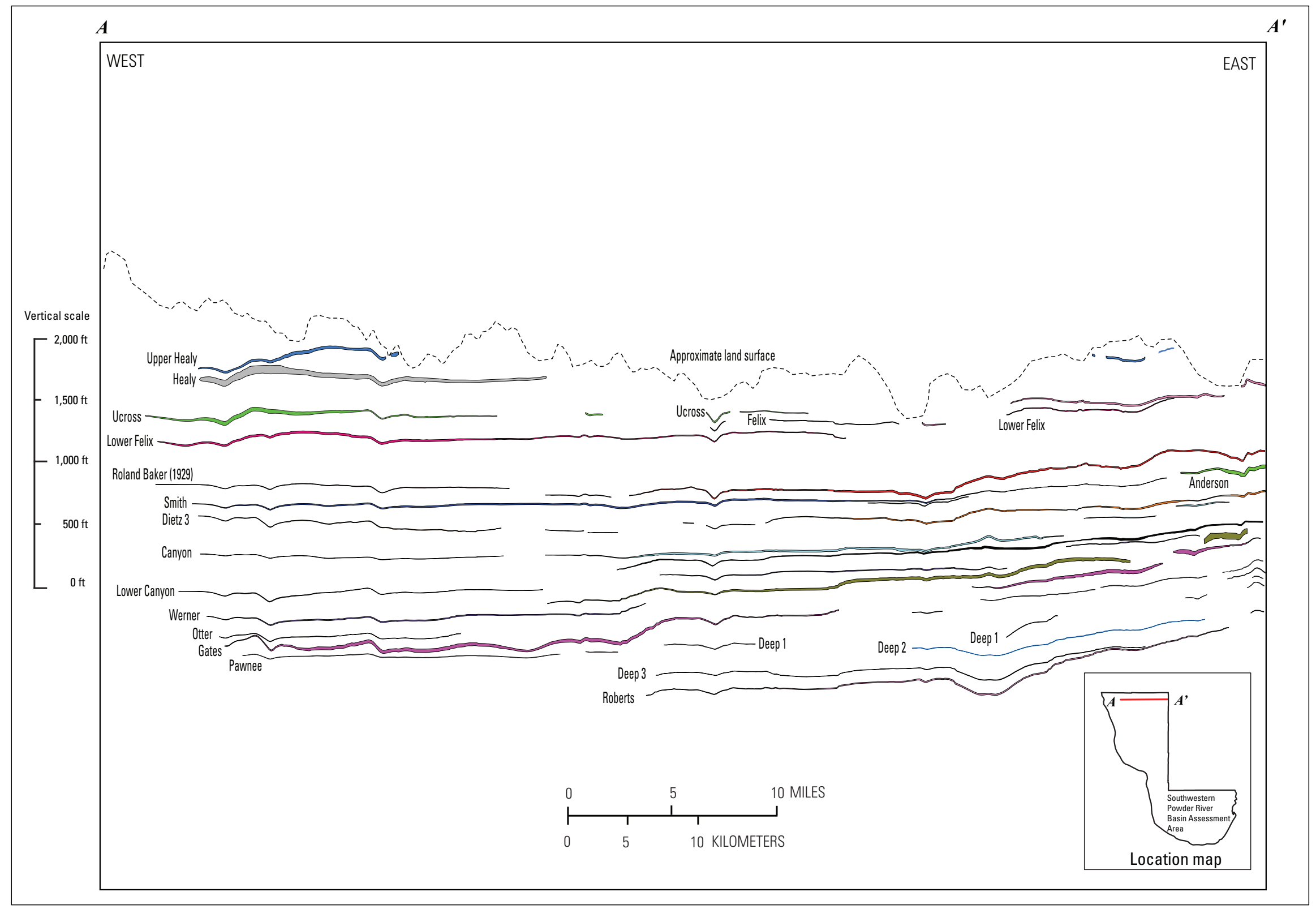

Figure 12. West-east cross section $A-A^{\prime}$ through the northern part of the southwestern Wyoming Powder River Basin assessment area showing subsurface coal beds (vertical exaggeration approximately $5,000 \times$ ). 


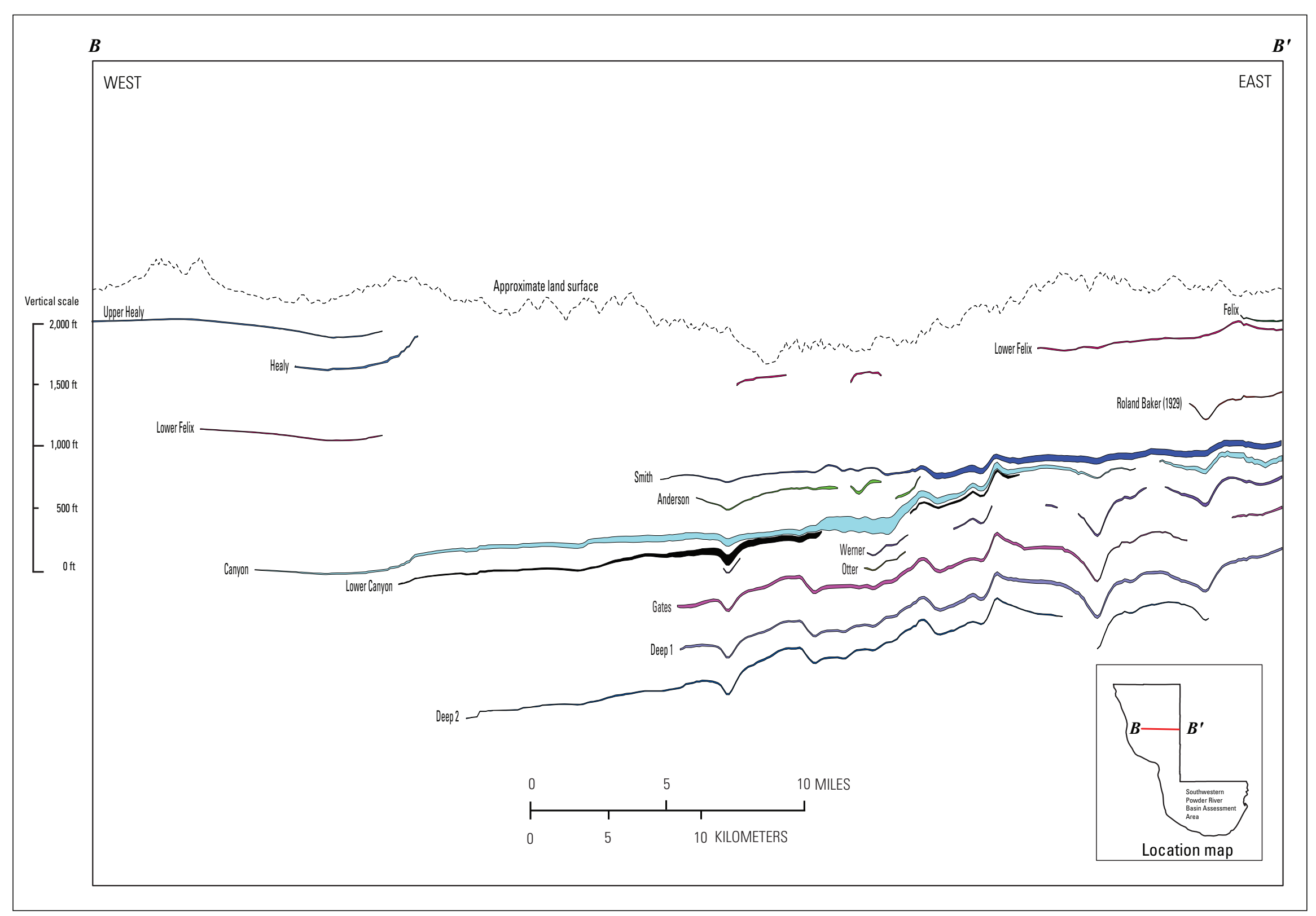

Figure 13. West-east cross section $B-B^{\prime}$ through the north-central part of the southwestern Wyoming Powder River Basin assessment area showing subsurface coal beds (vertical exaggeration approximately $5,000 \times$ ). 


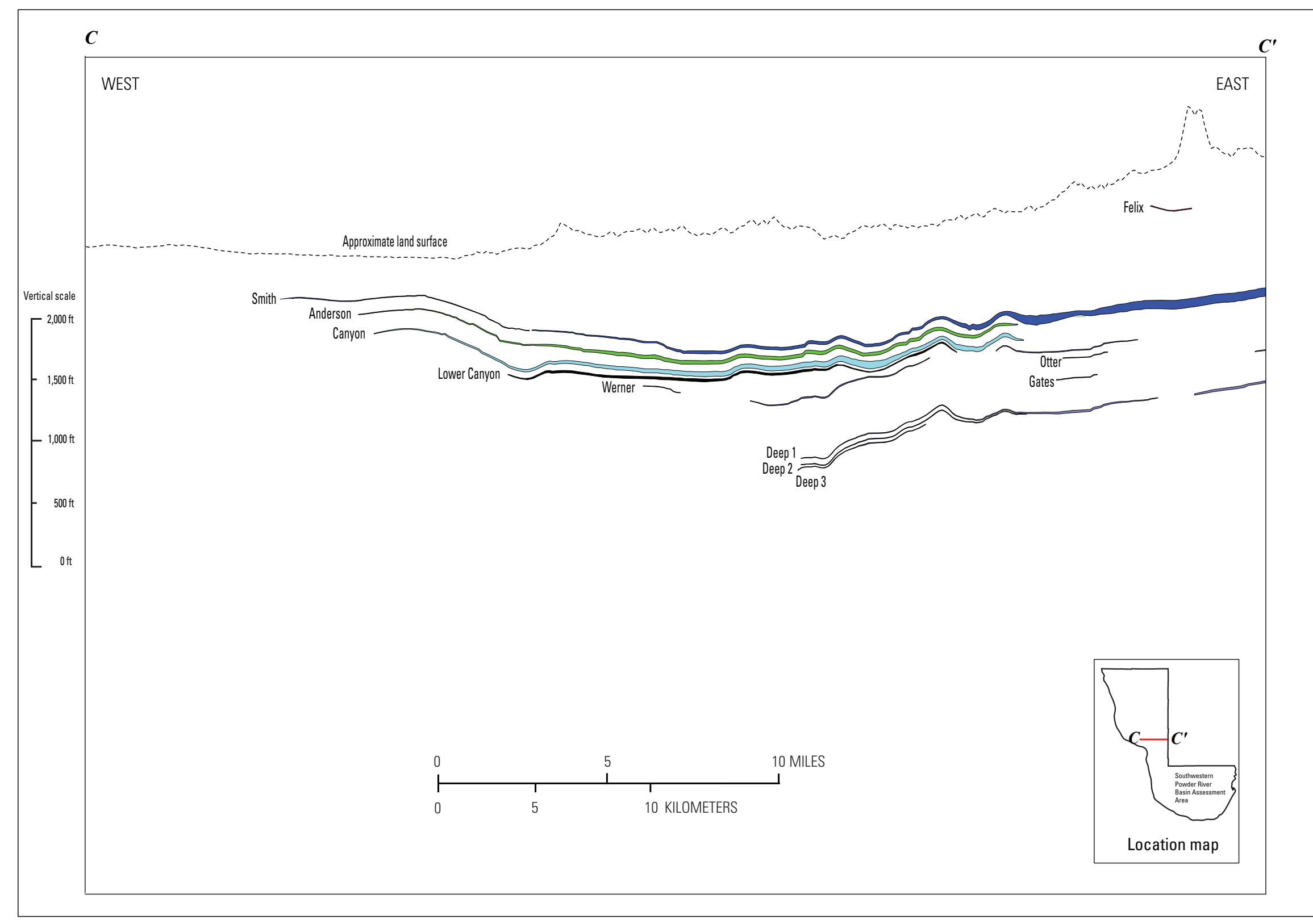

Figure 14. West-east cross section $C-C^{\prime}$ through the central part of the southwestern Wyoming Powder River

Basin assessment area showing subsurface coal beds (vertical exaggeration approximately 5,000 $\times$ ). 


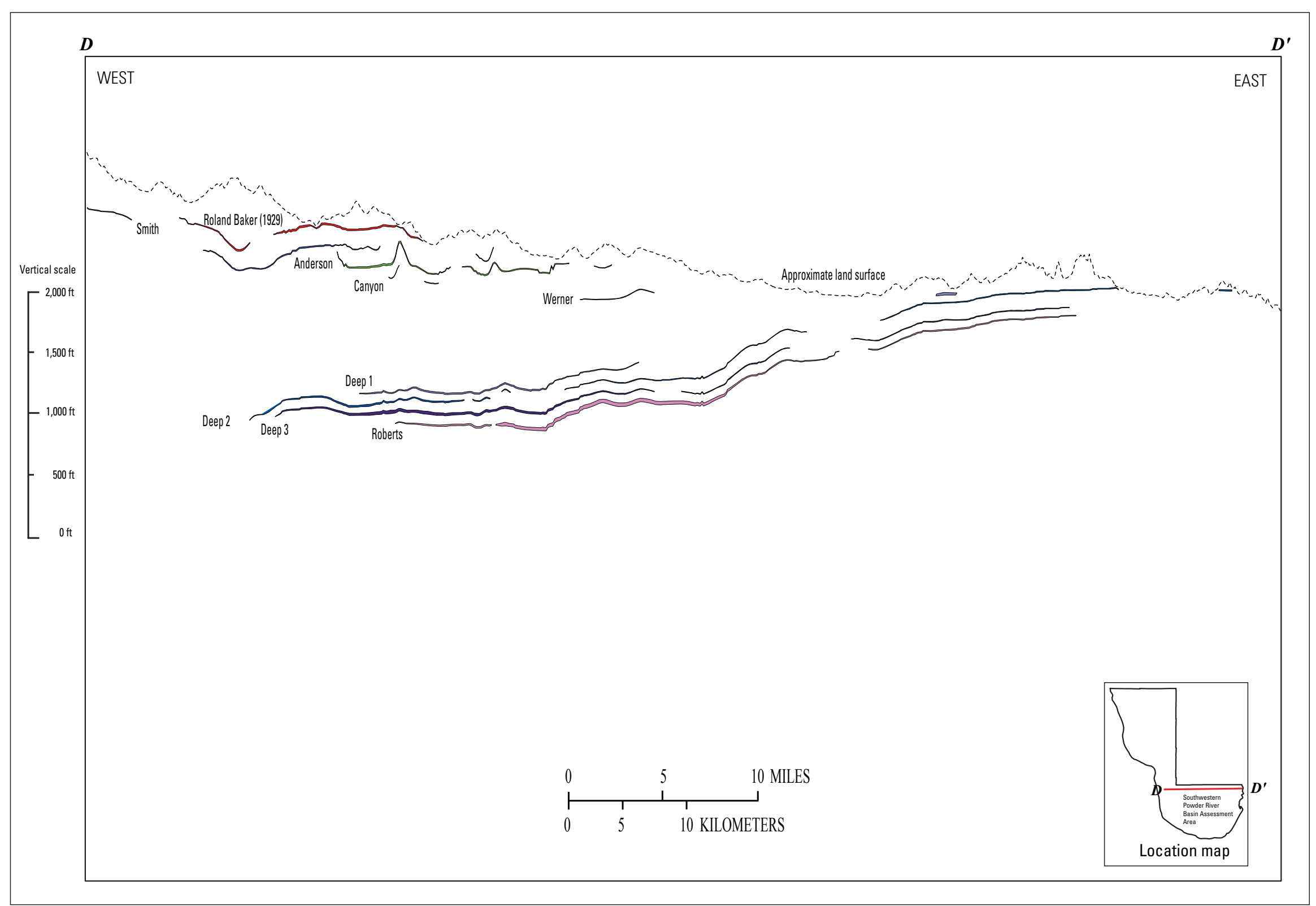

Figure 15. West-east cross section $D-D^{\prime}$ through the south-central part of the southwestern Wyoming Powder River Basin assessment area showing subsurface coal beds (vertical exaggeration approximately $5,000 \times$ ). 


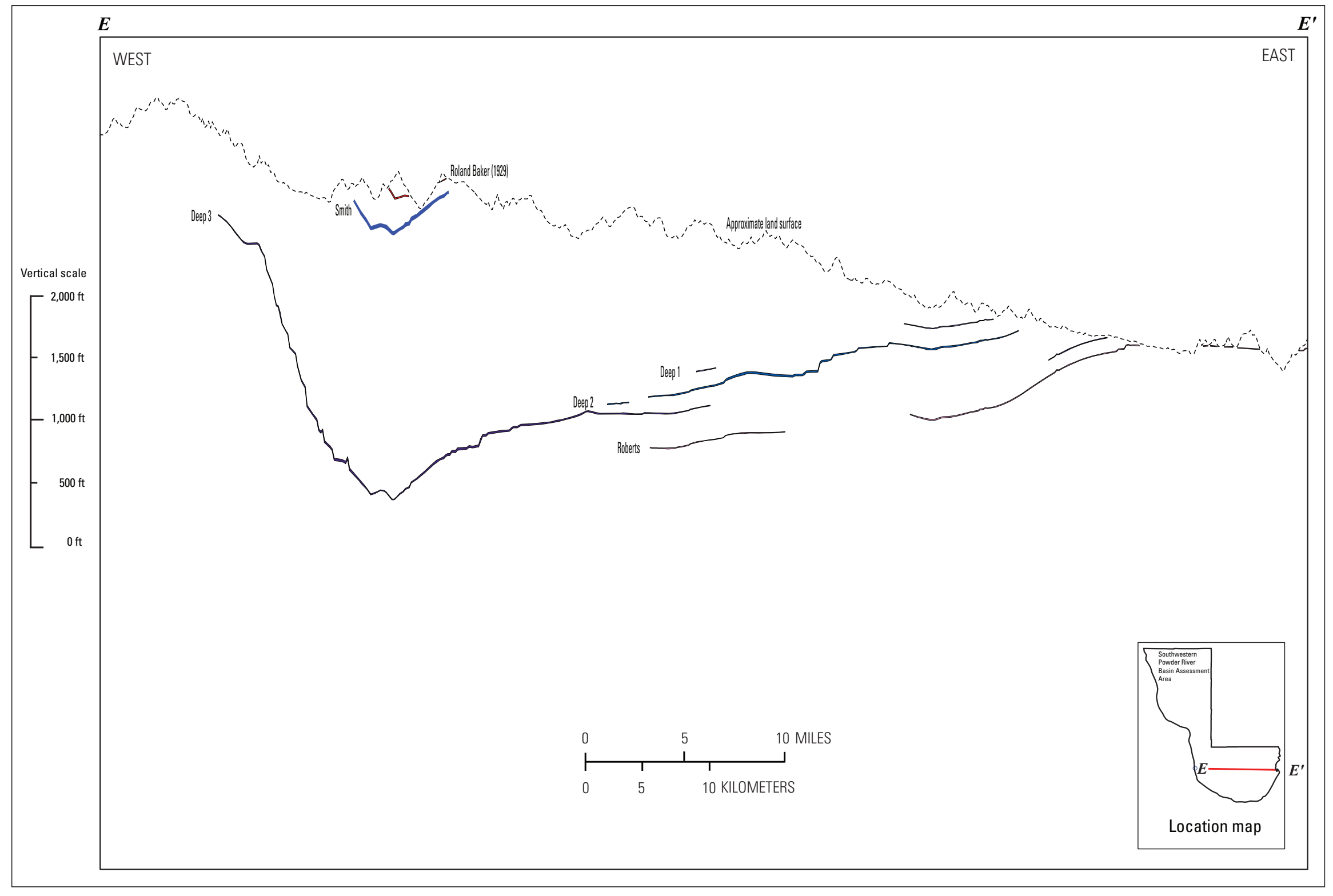

Figure 16. West-east cross section $E-E^{\prime}$ through the southern part of the southwestern Wyoming Powder River Basin assessment area showing subsurface coal beds (vertical exaggeration approximately 5,000 ×). 


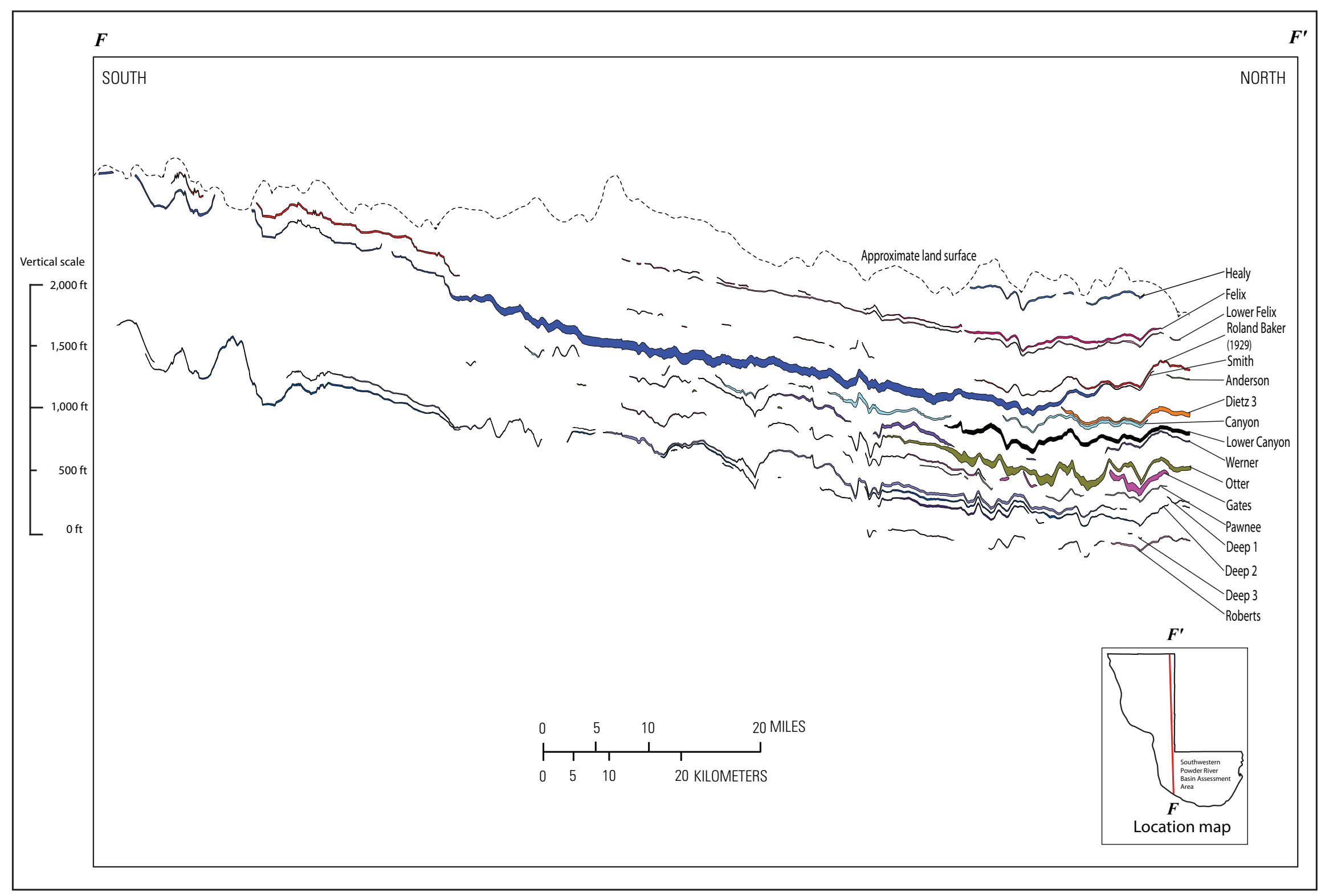

Figure 17. South-north cross section $F-F^{\prime}$ through the central part of the southwestern Wyoming Powder River Basin assessment area showing subsurface coal beds (vertical exaggeration approximately 5,000 ×). 


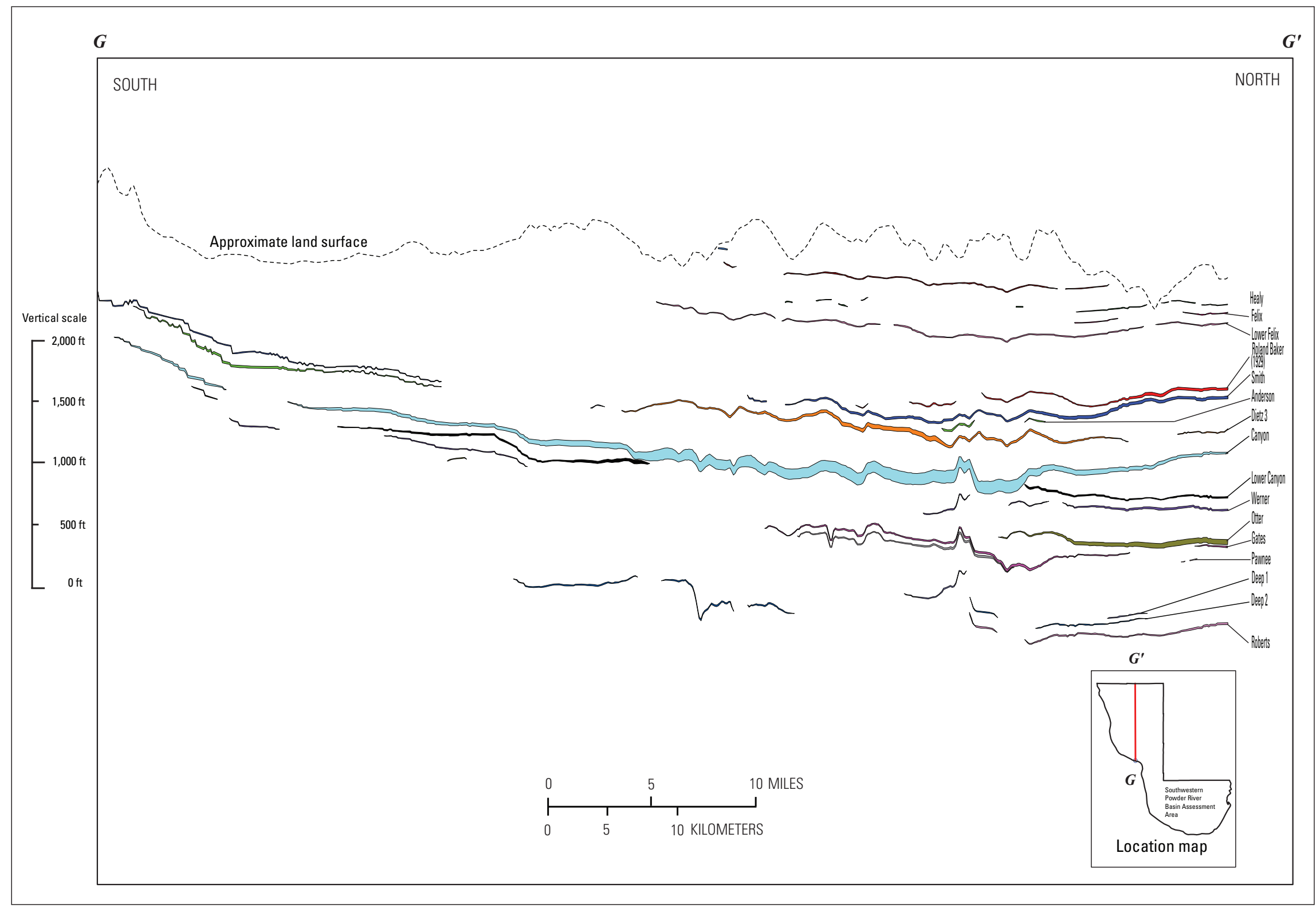

Figure 18. South-north cross section $G-G^{\prime}$ through the northern part of the southwestern Wyoming Powder River Basin assessment area showing subsurface coal beds (vertical exaggeration approximately 5,000 $\times$ ). 


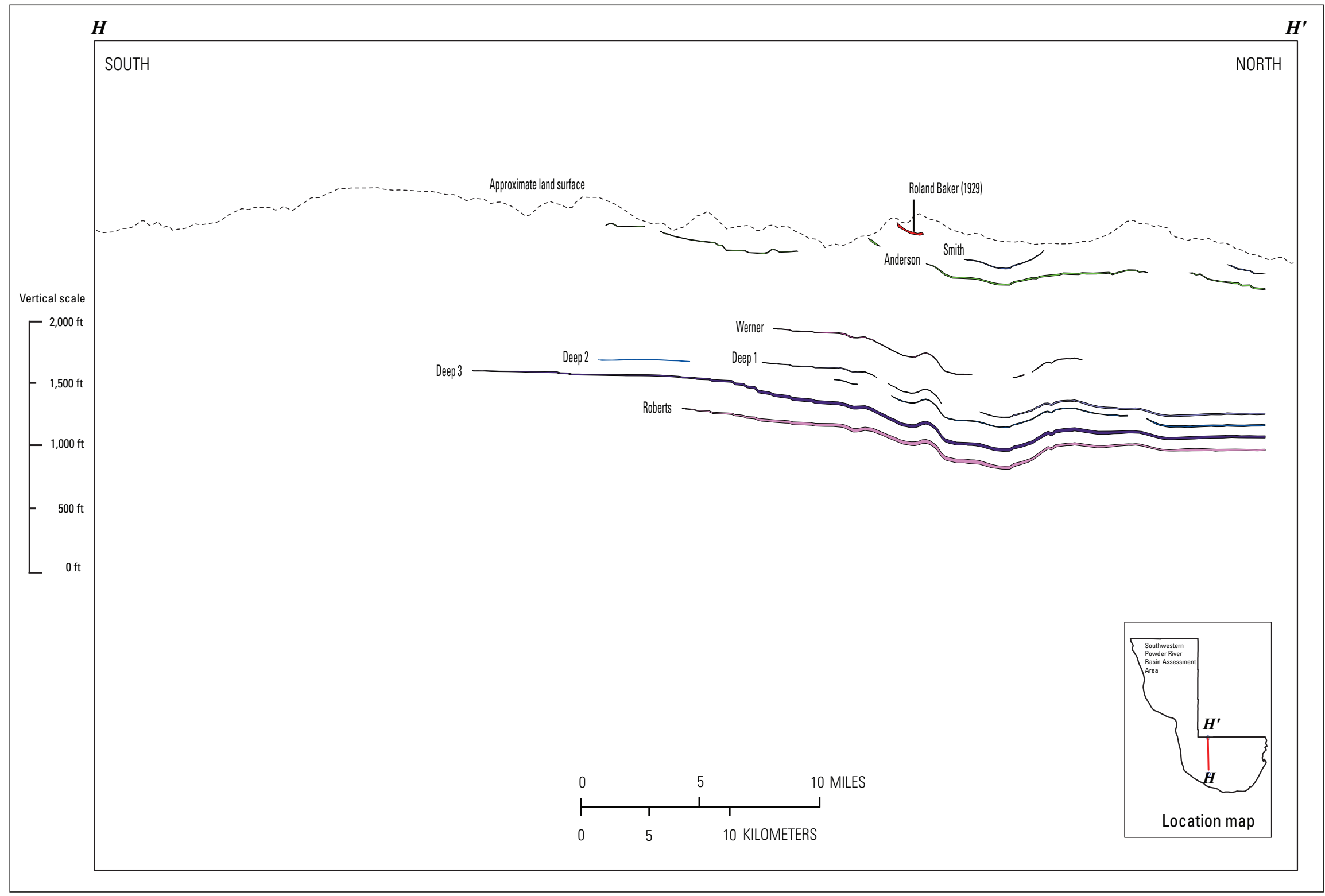

Figure 19. South-north cross section $H-H^{\prime}$ through the south-central part of the southwestern Wyoming Powder River Basin assessment area showing subsurface coal beds (vertical exaggeration approximately $5,000 \times$ ). 


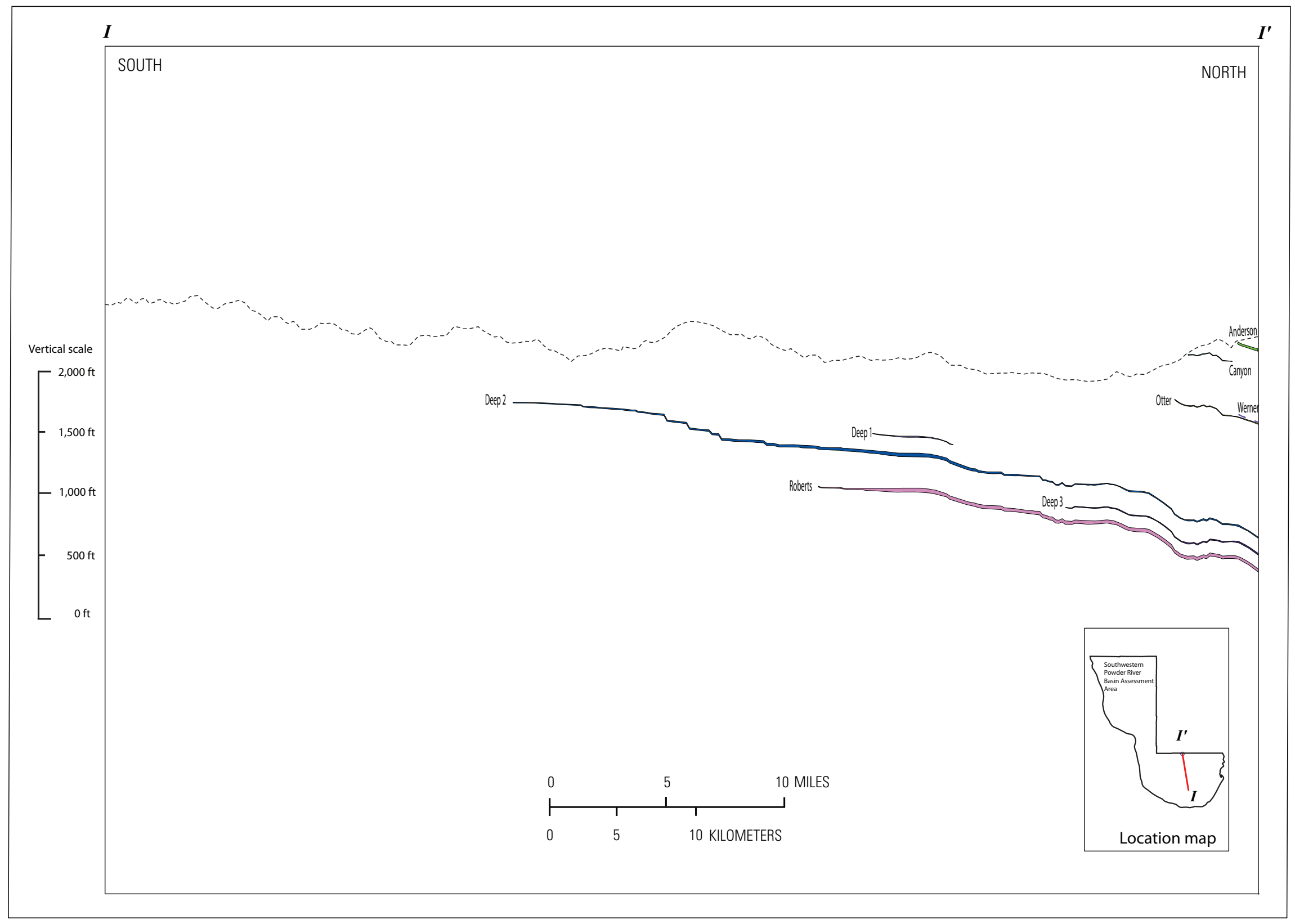

Figure 20. South-north cross section $I-I^{\prime}$ through the south-eastern part of the southwestern Wyoming Powder River Basin assessment area showing subsurface coal beds (vertical exaggeration approximately 5,000 ×). 


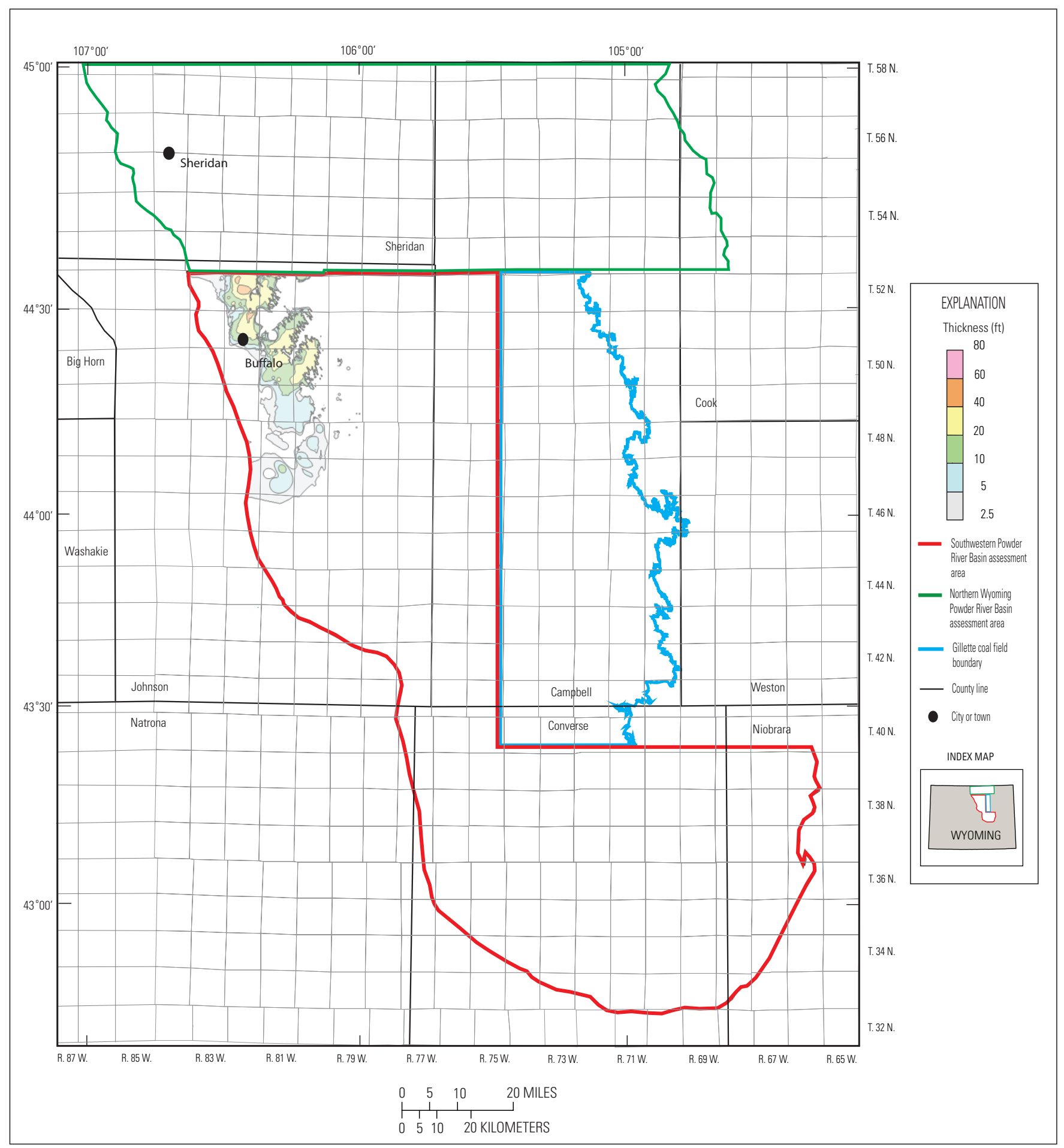

Figure 21. Isopach map of the Upper Healy coal bed showing extent of resources greater than $2.5 \mathrm{ft}$ thick within the Southwestern Powder River Basin assessment area. 


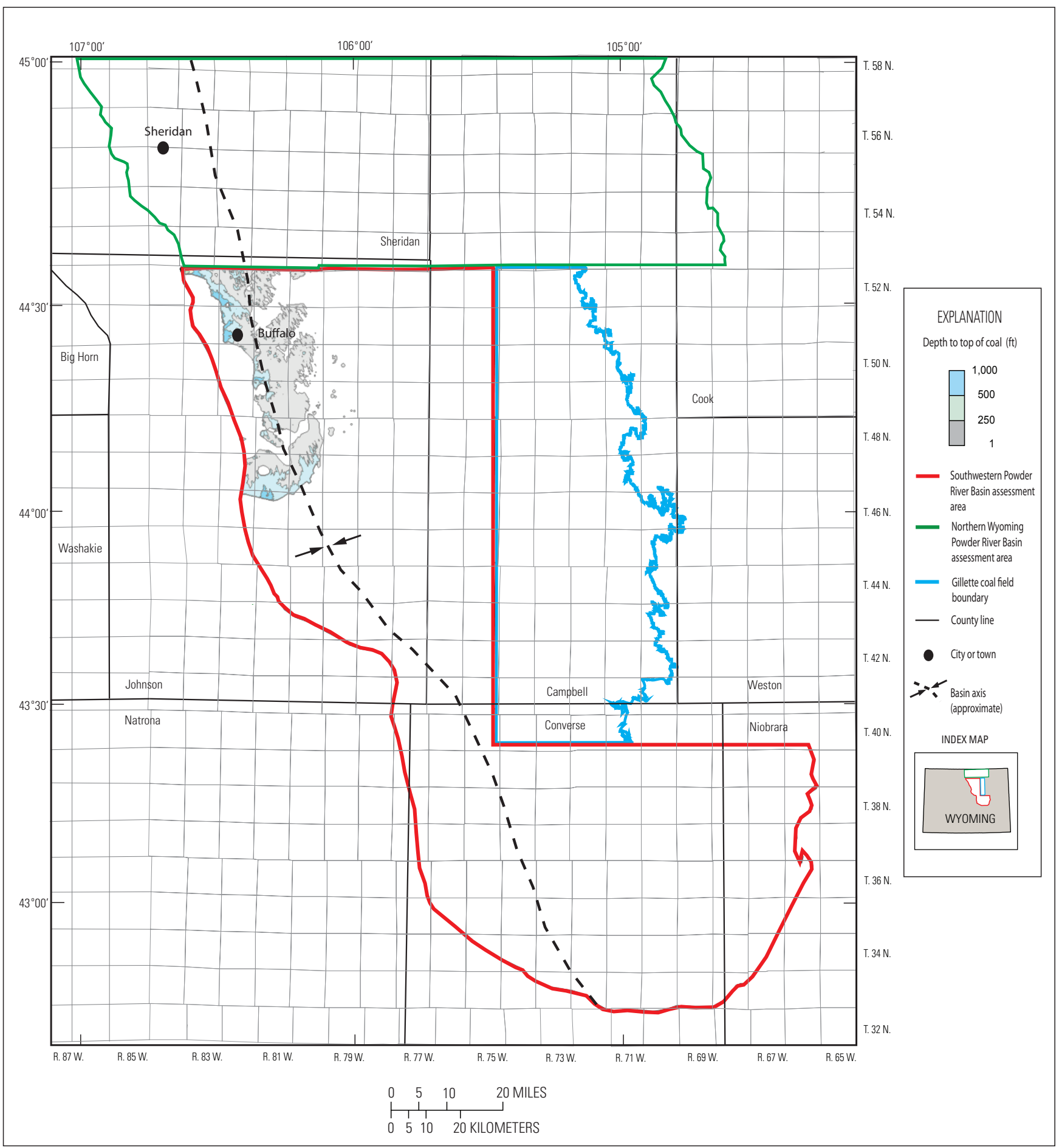

Figure 22. Depth to the top of the Upper Healy coal bed within the Southwestern Powder River Basin assessment area. 


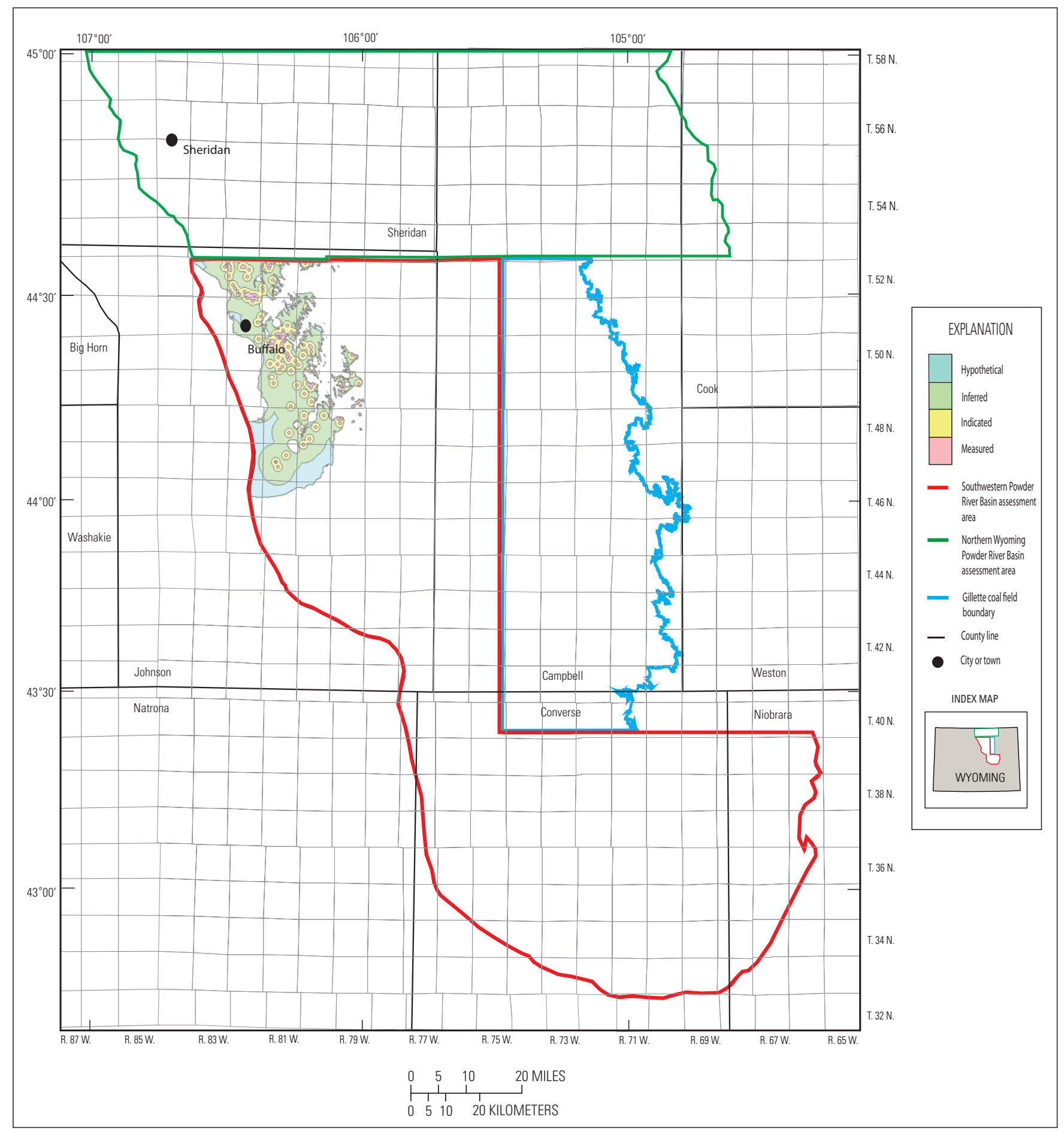

Figure 23. Coal resource reliability categories for the Upper Healy coal bed within the Southwestern Powder River Basin assessment area. 


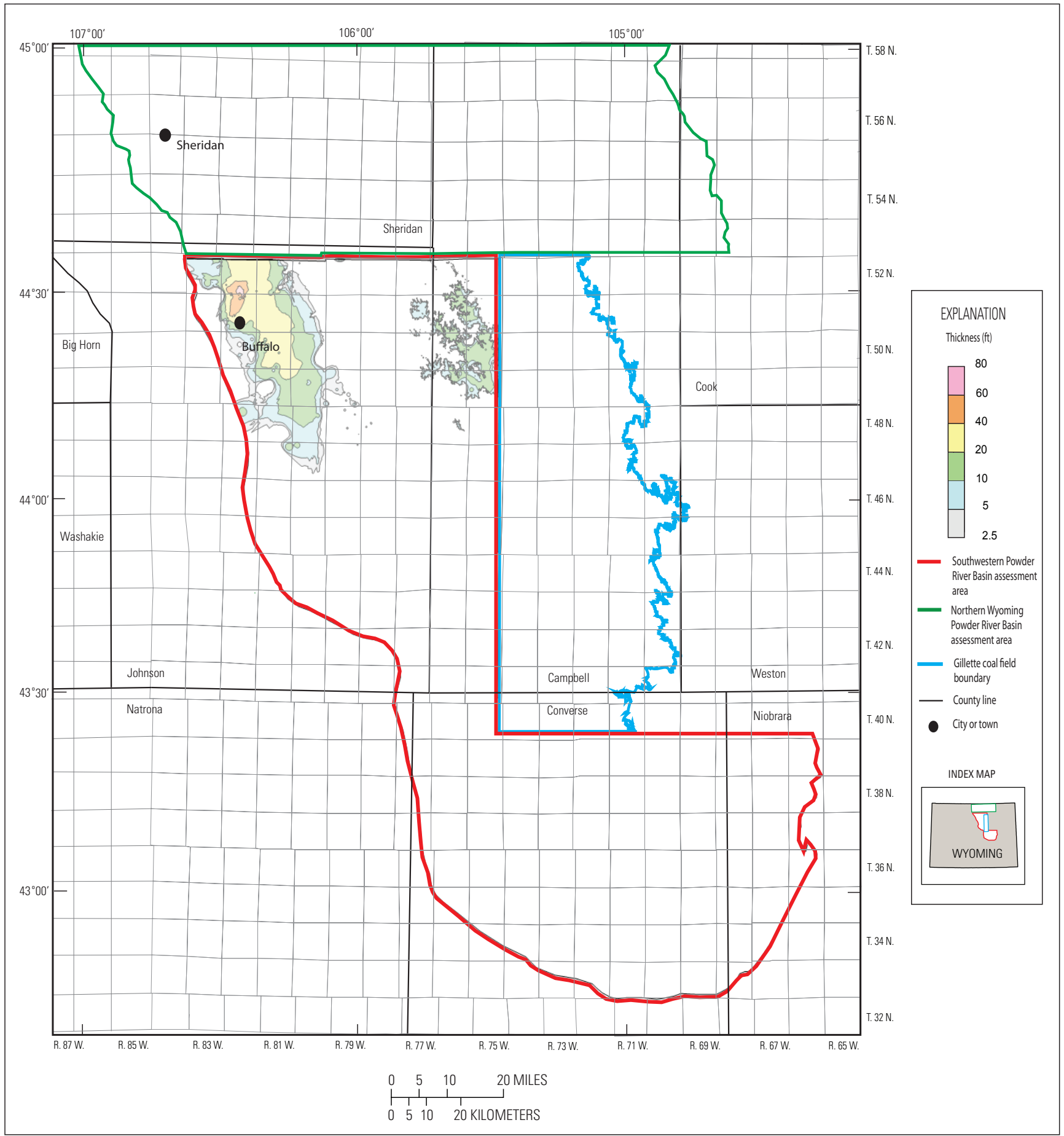

Figure 24. Isopach map of the Healy coal bed showing extent of resources greater than $2.5 \mathrm{ft}$ thick within the Southwestern Powder River Basin assessment area. 


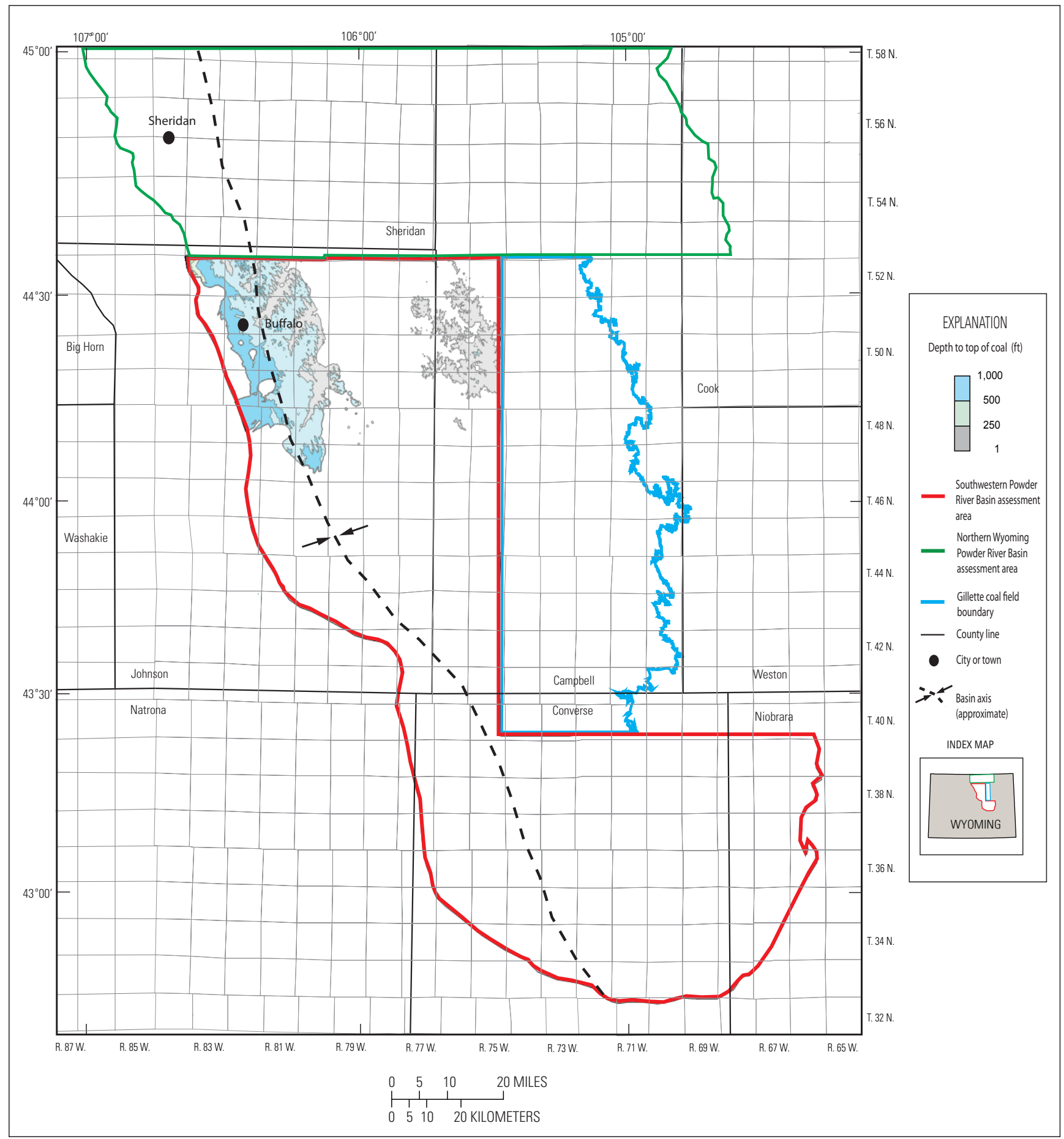

Figure 25. Depth to the top of the Healy coal bed within the Southwestern Powder River Basin assessment area. 


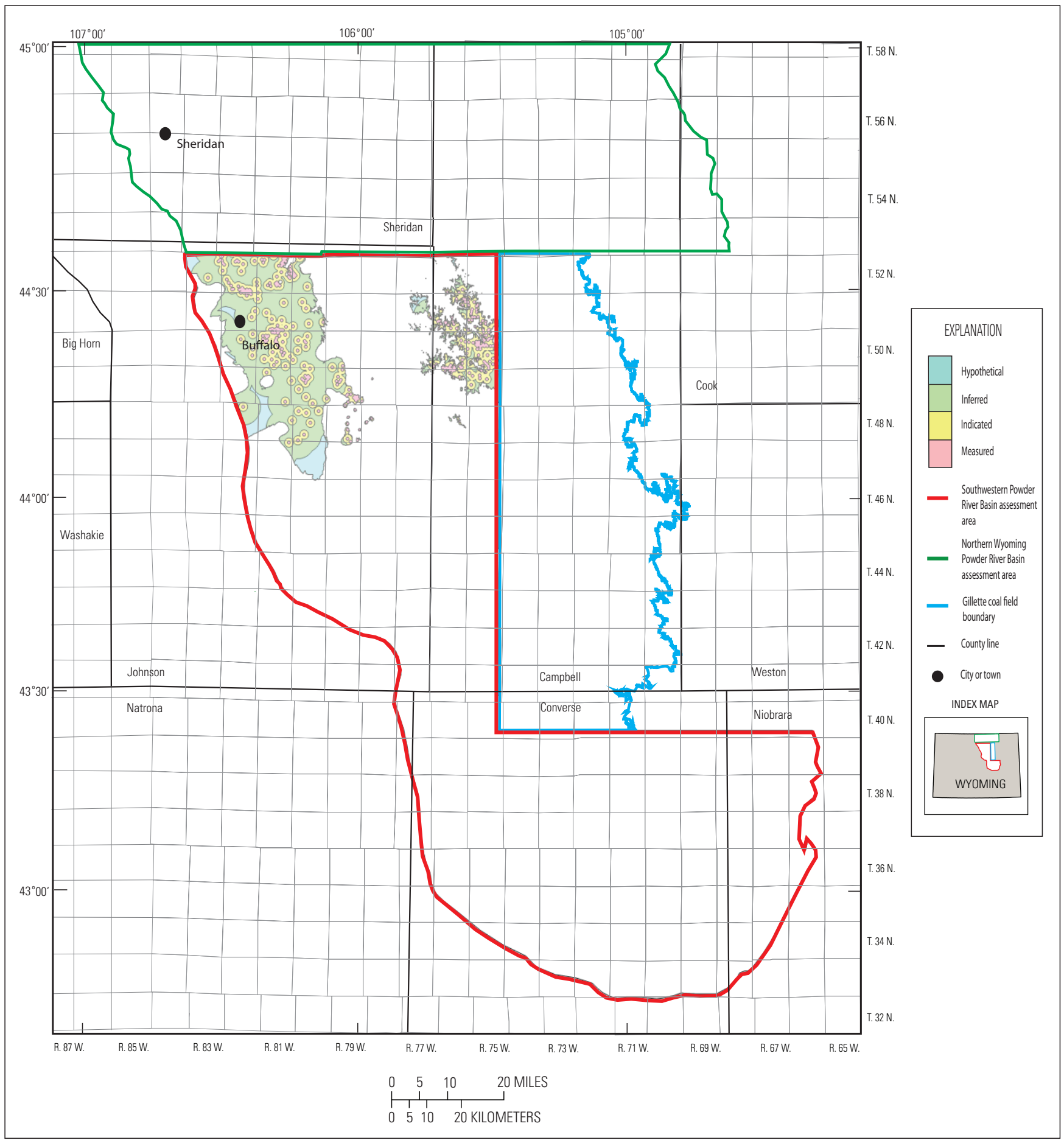

Figure 26. Coal resource reliability categories for the Healy coal bed within the Southwestern Powder River Basin assessment area. 


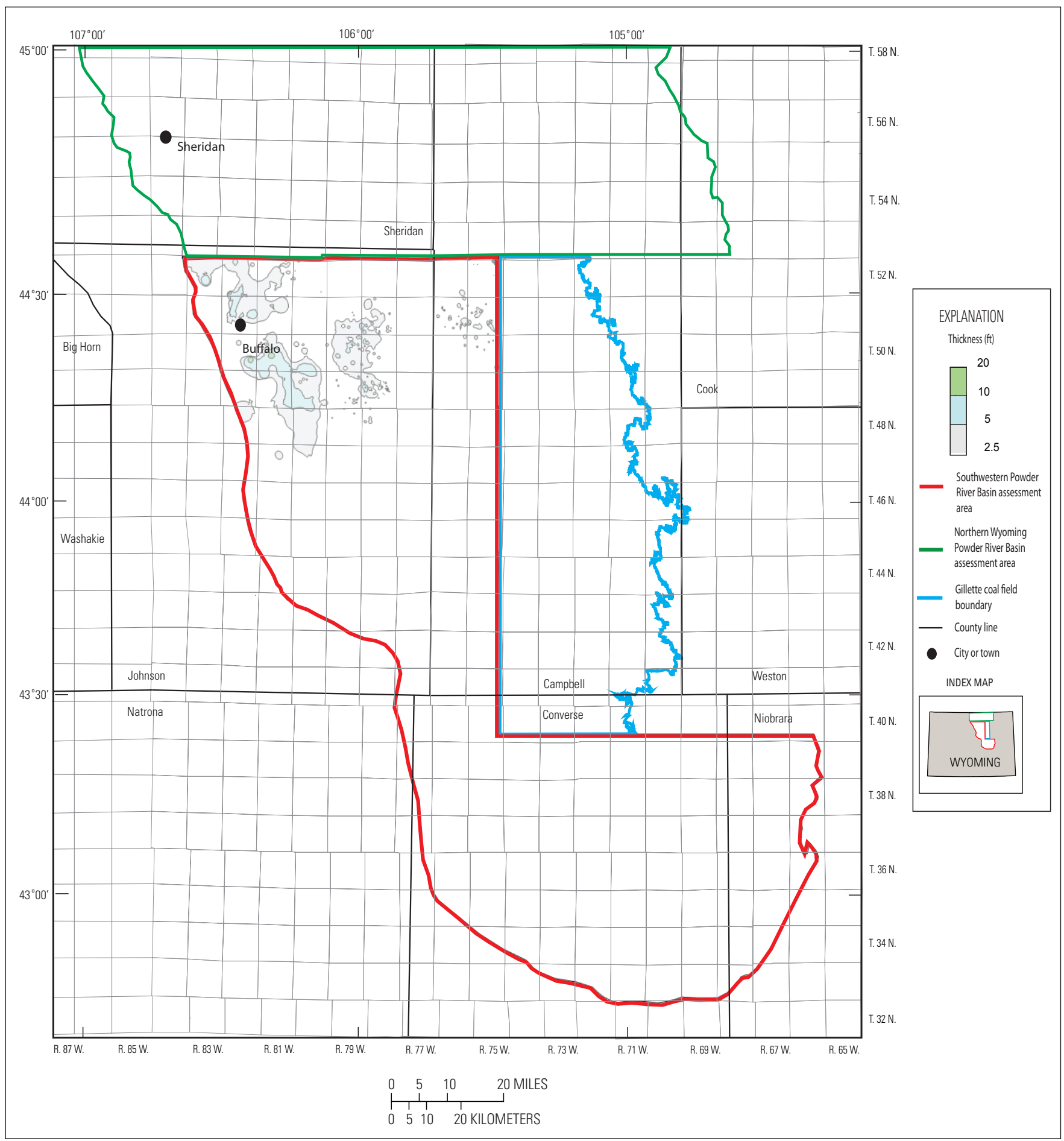

Figure 27. Isopach map of the Murray coal bed showing extent of resources greater than $2.5 \mathrm{ft}$ thick within the Southwestern Powder River Basin assessment area. 


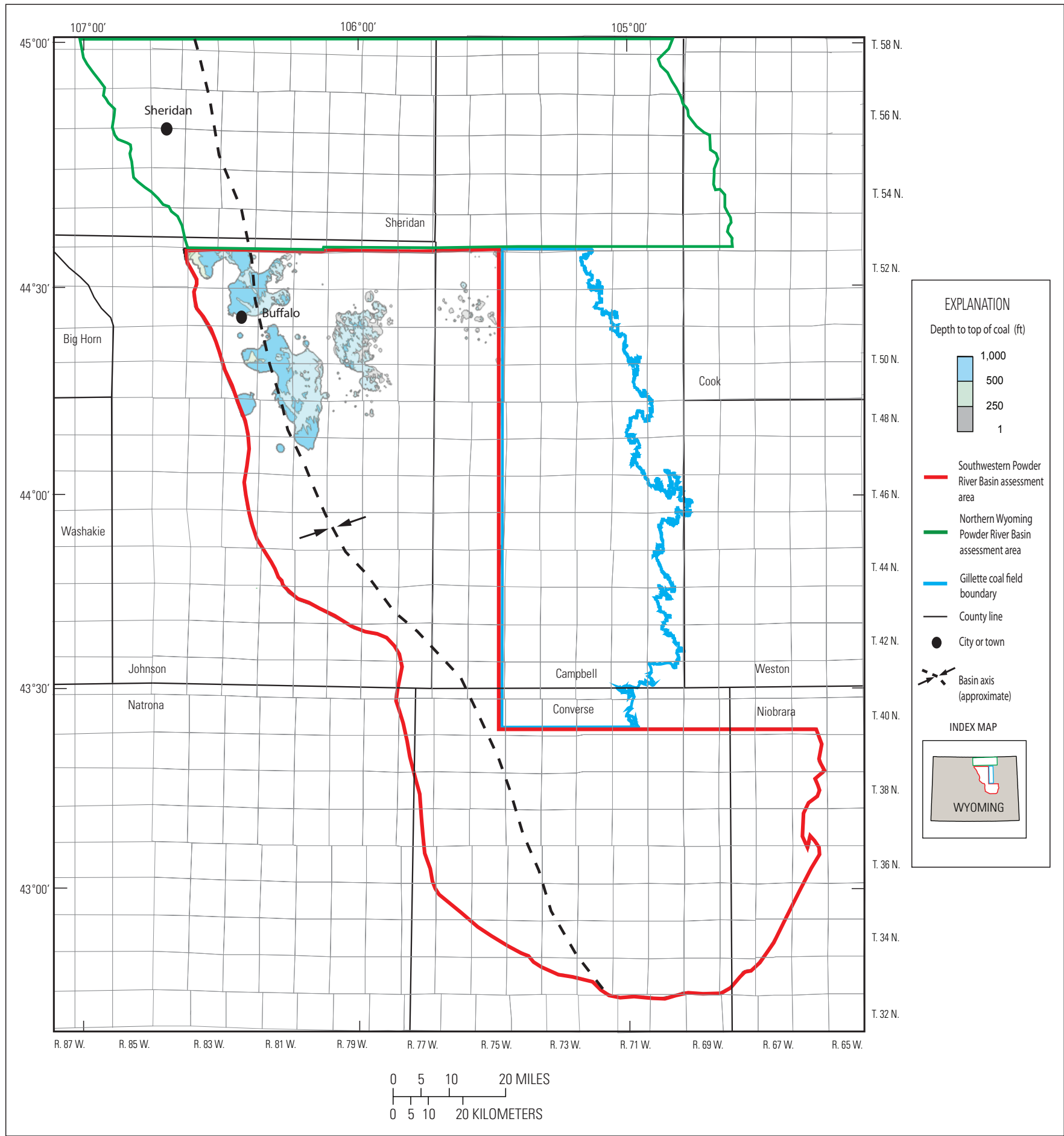

Figure 28. Depth to the top of the Murray coal bed within the Southwestern Powder River Basin assessment area. 


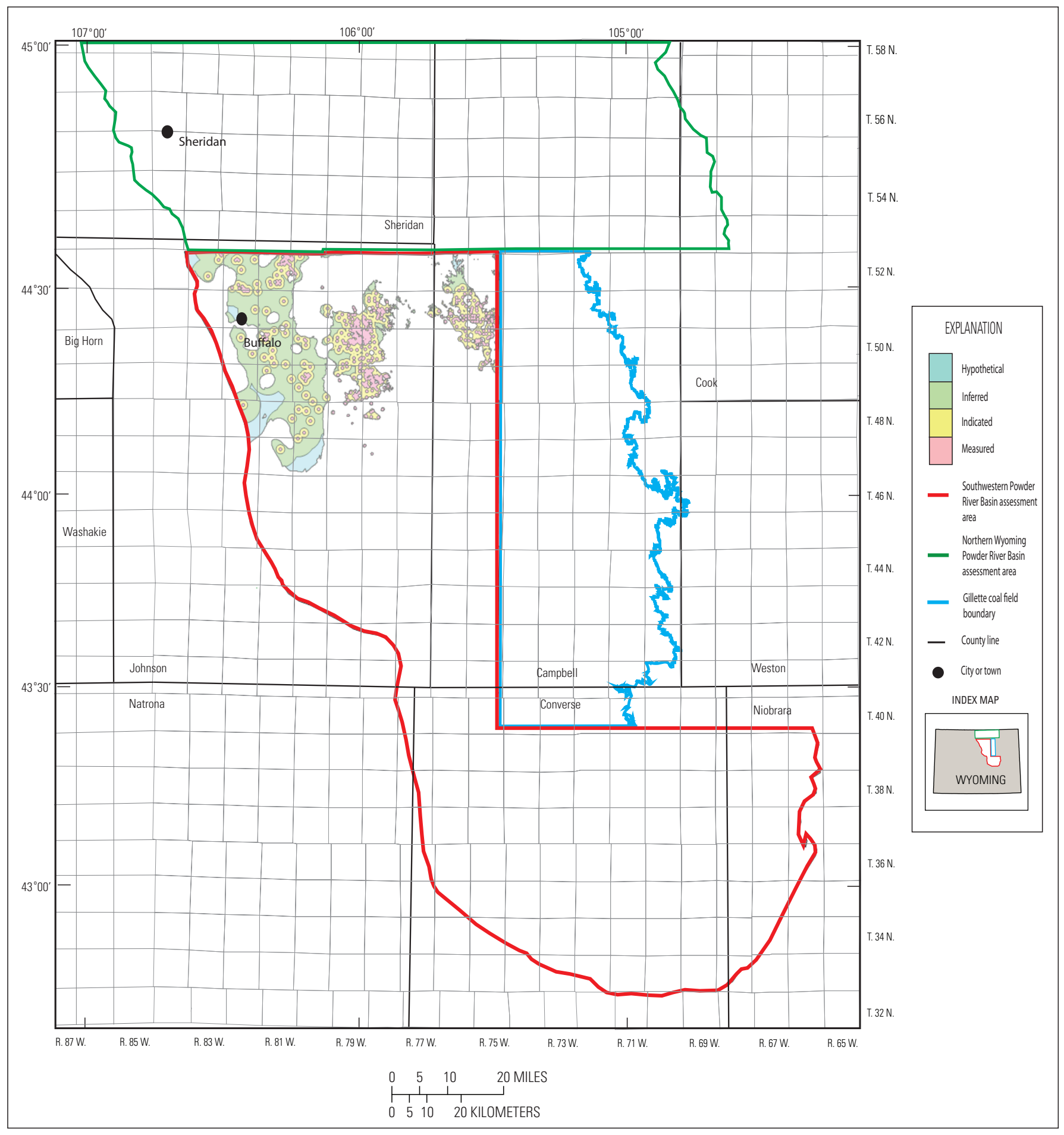

Figure 29. Coal resource reliability categories for the Murray coal bed within the Southwestern Powder River Basin assessment area. 


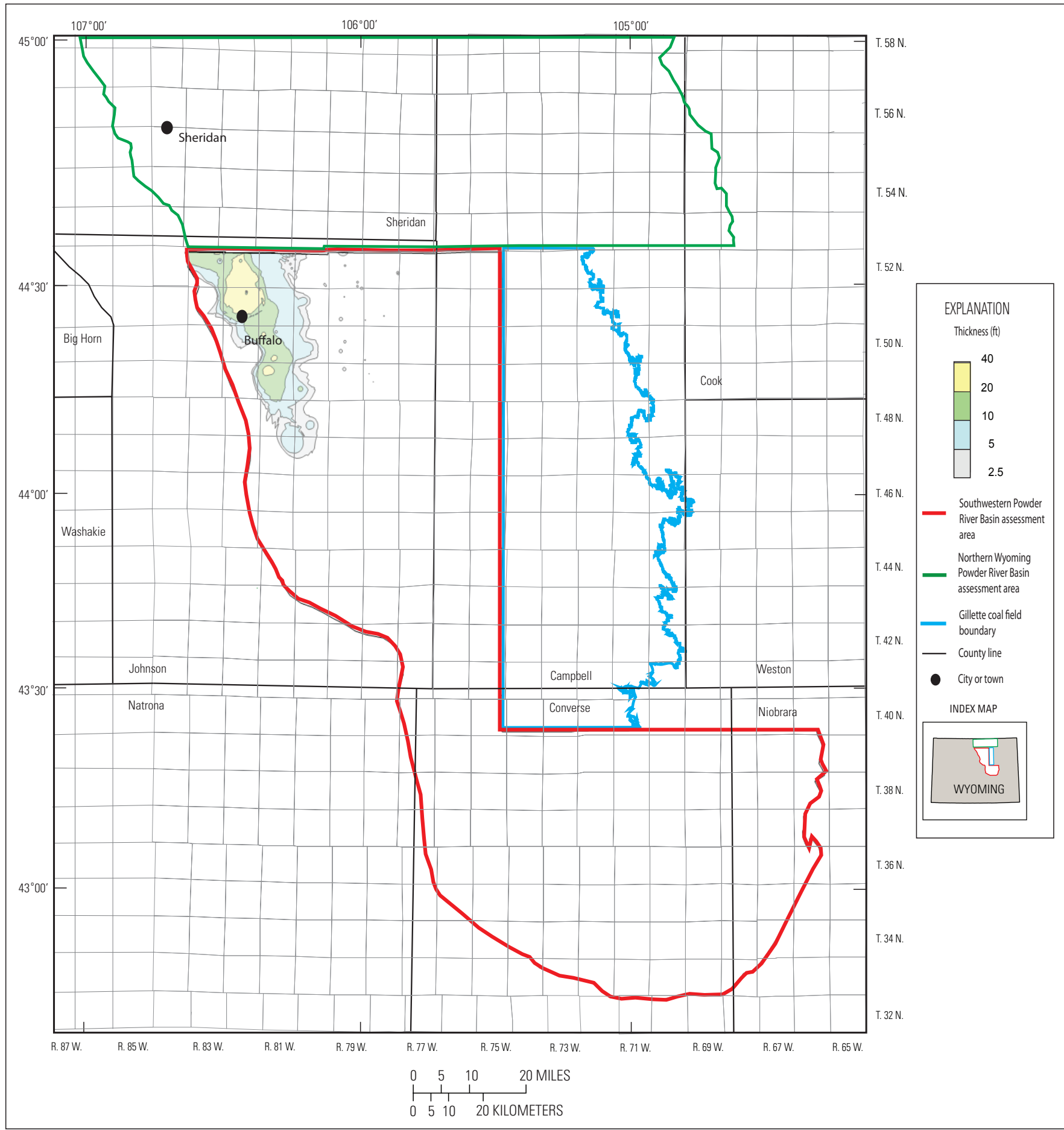

Figure 30. Isopach map of the Ucross coal bed showing extent of resources greater than $2.5 \mathrm{ft}$ thick within the Southwestern Powder River Basin assessment area. 


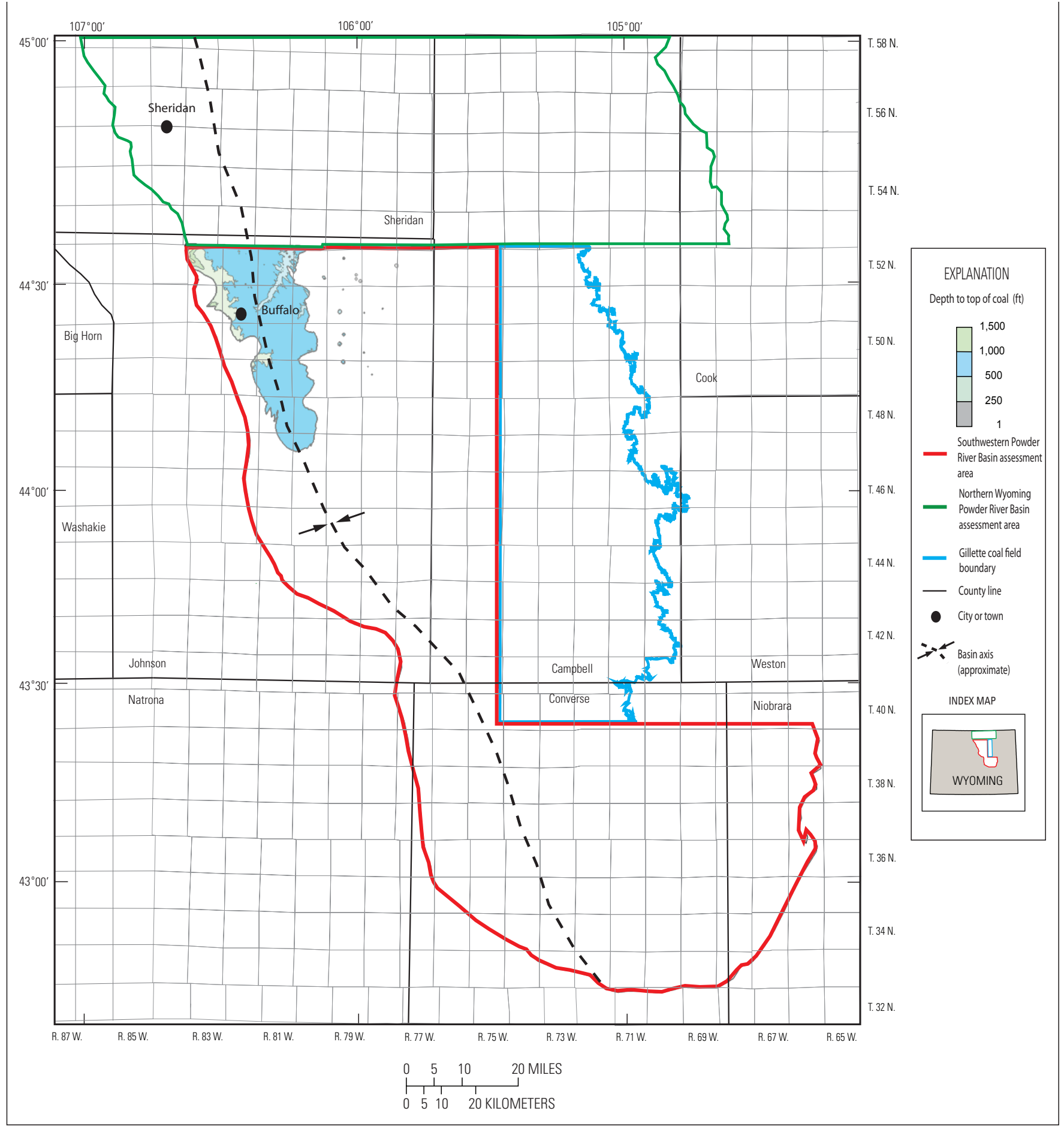

Figure 31. Depth to the top of the Ucross coal bed within the Southwestern Powder River Basin assessment area. 


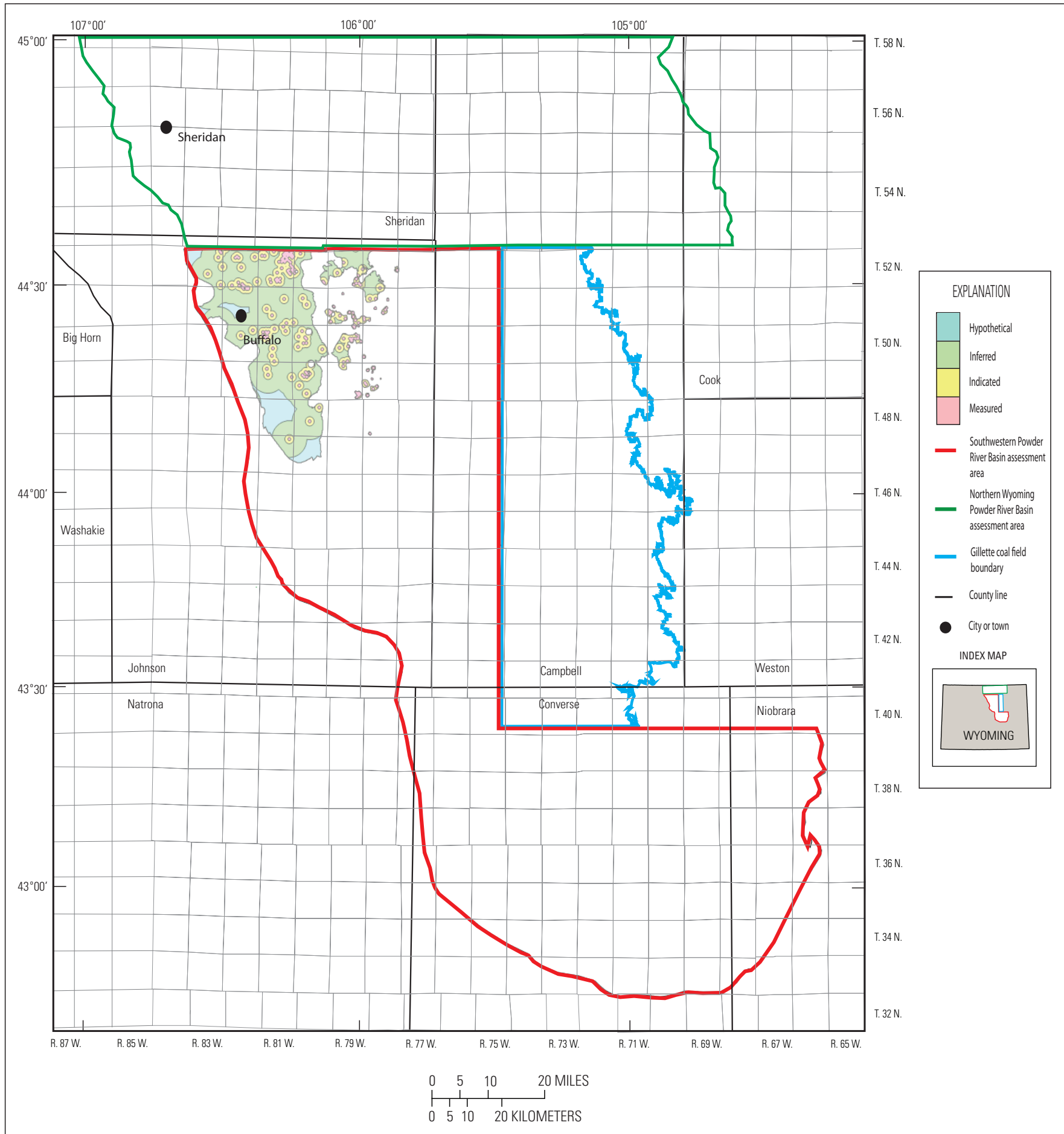

Figure 32. Coal resource reliability categories for the Ucross coal bed within the Southwestern Powder River Basin assessment area. 


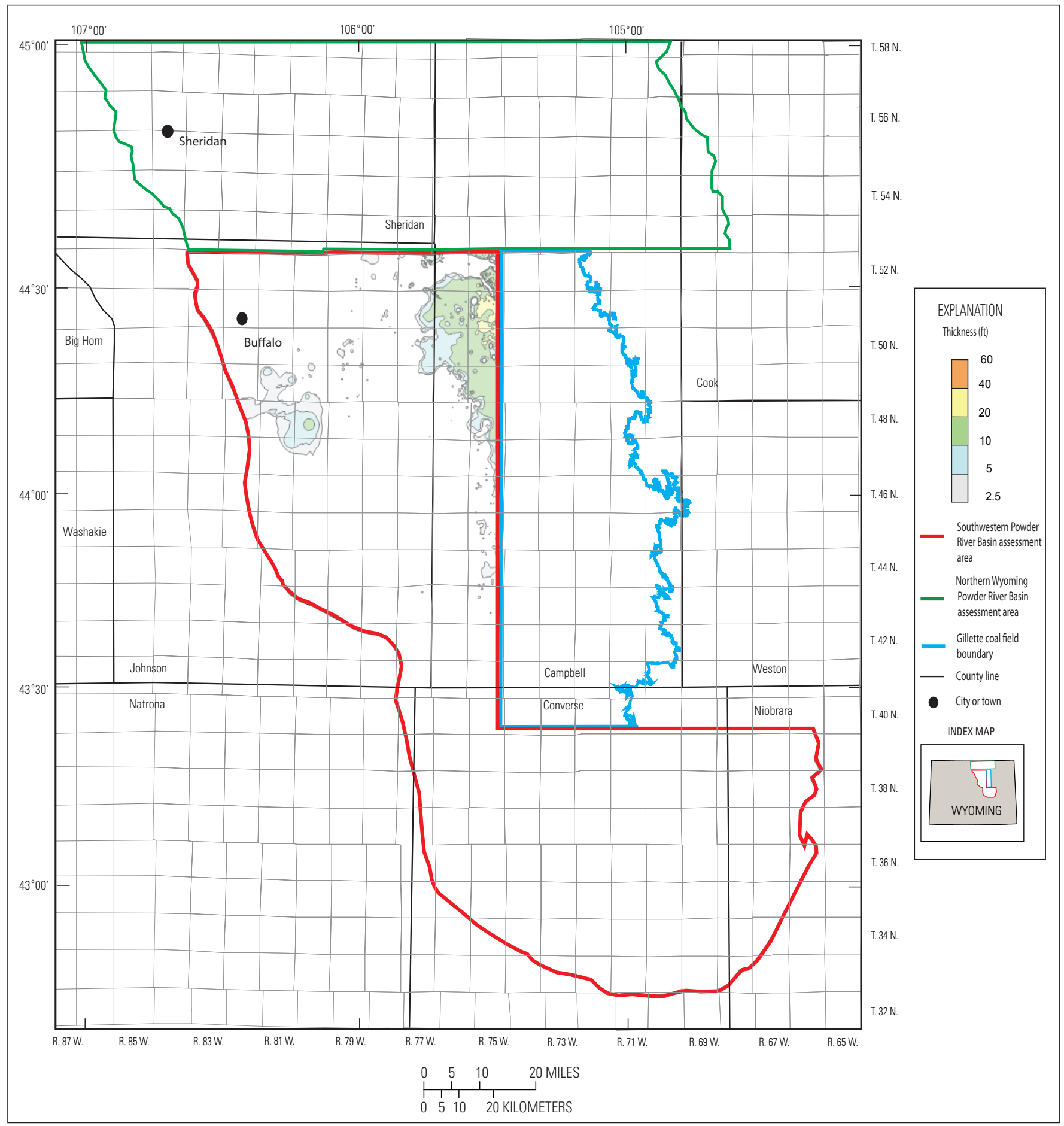

Figure 33. Isopach map of the Felix coal bed showing extent of resources greater than $2.5 \mathrm{ft}$ thick within the Southwestern Powder River Basin assessment area. 


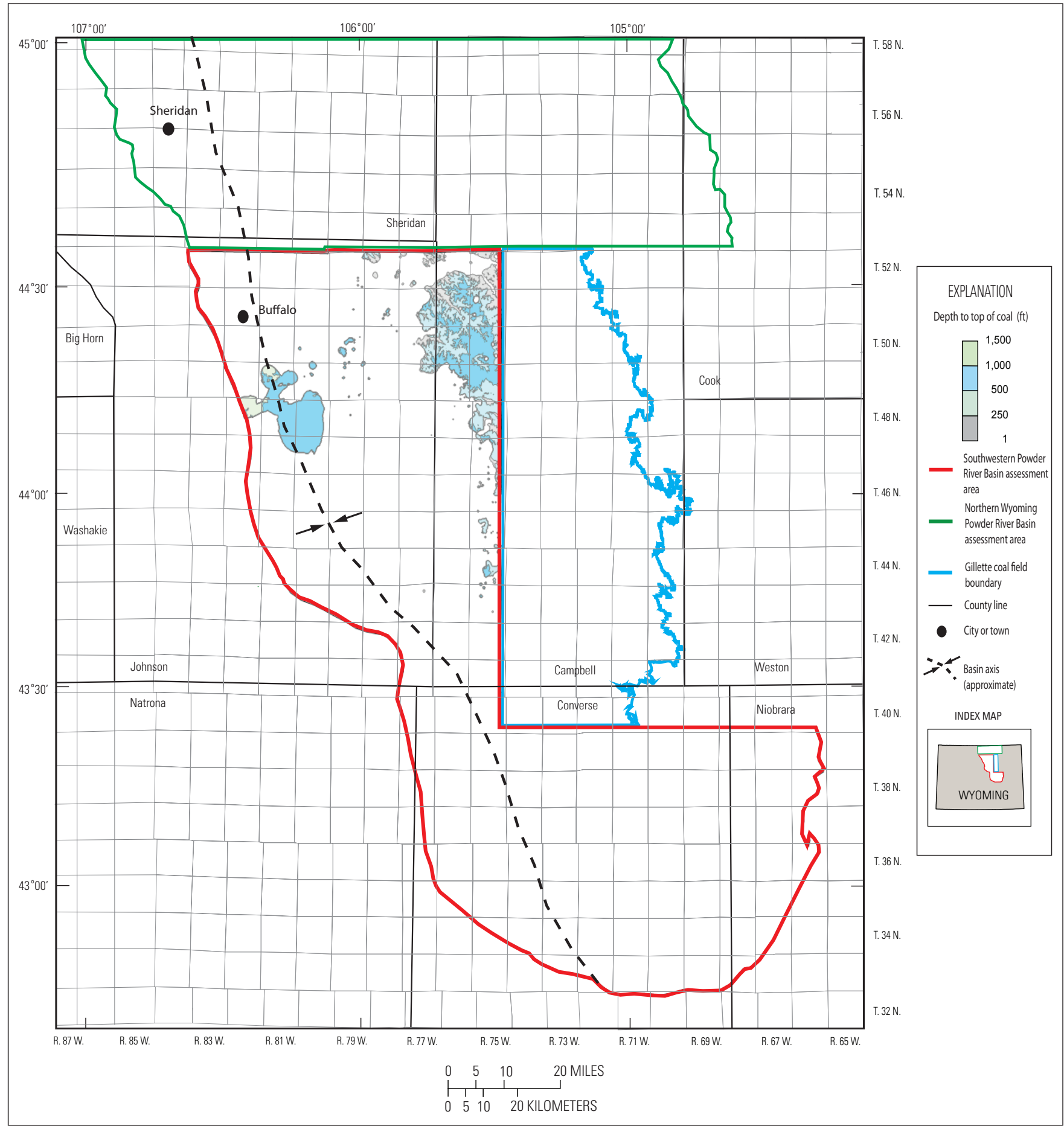

Figure 34. Depth to the top of the Felix coal bed within the Southwestern Powder River Basin assessment area. 


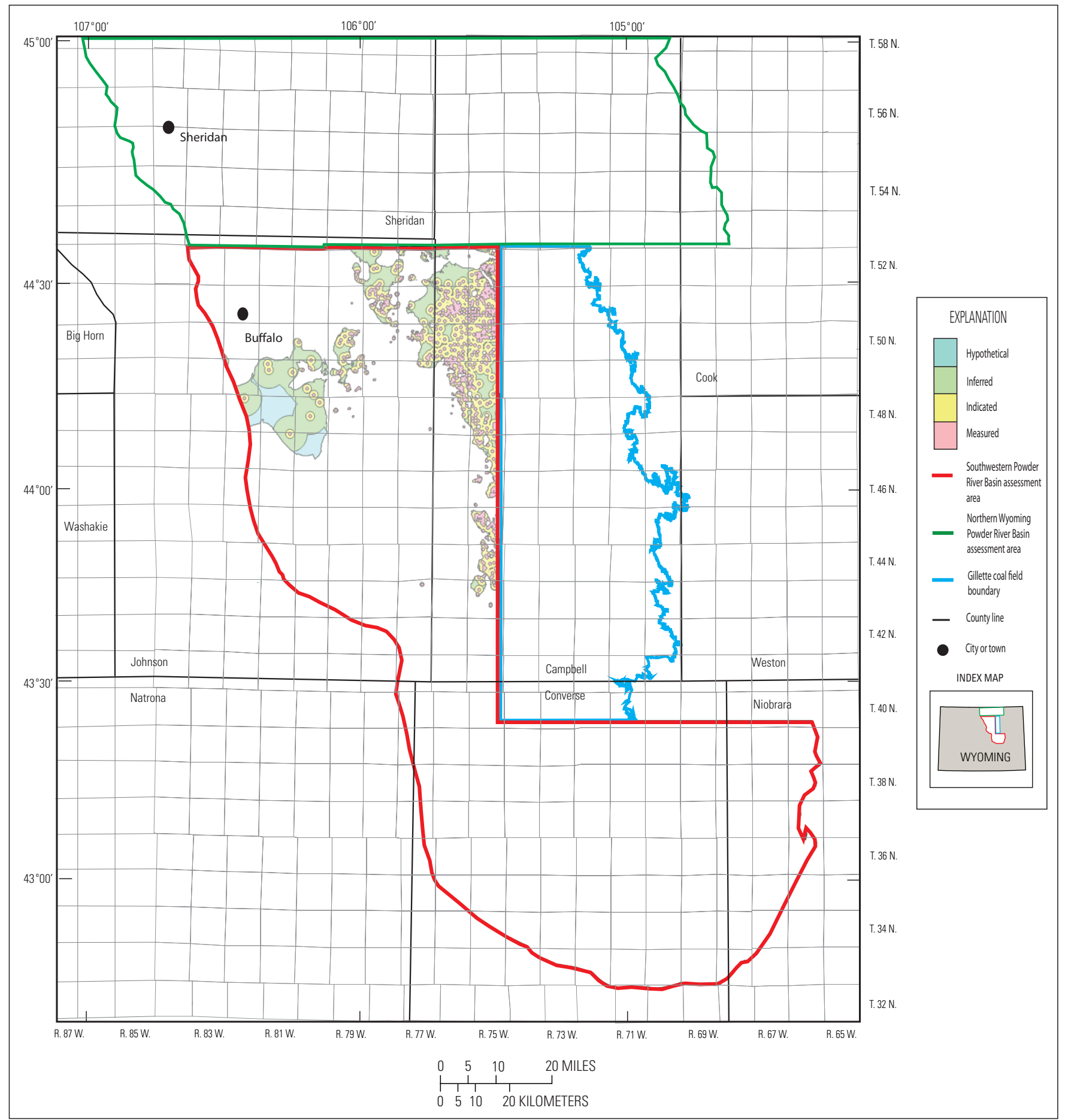

Figure 35. Coal resource reliability categories for the Felix coal bed within the Southwestern Powder River Basin assessment area. 


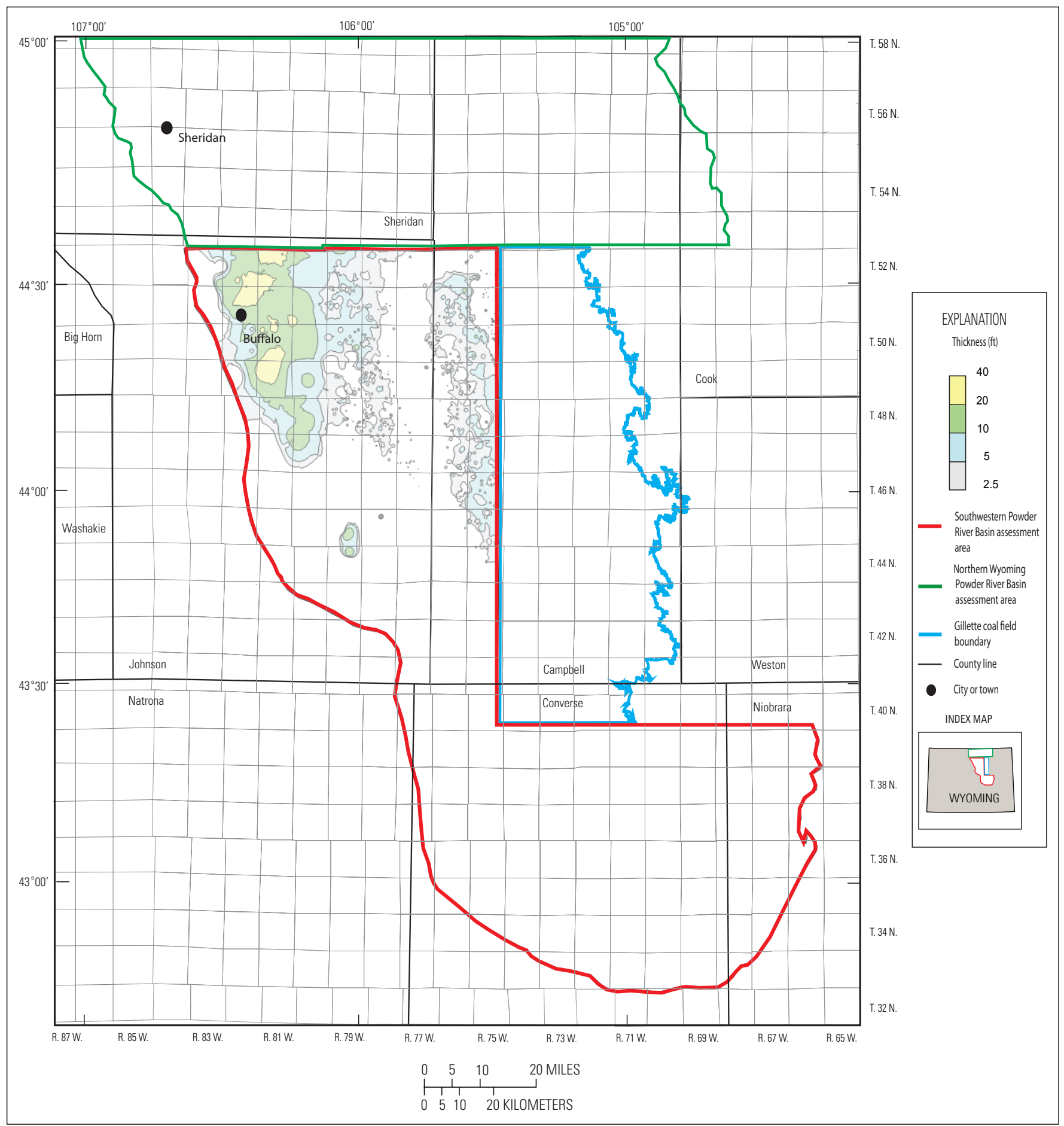

Figure 36. Isopach map of the Lower Felix coal bed showing extent of resources greater than $2.5 \mathrm{ft}$ thick within the Southwestern Powder River Basin assessment area. 


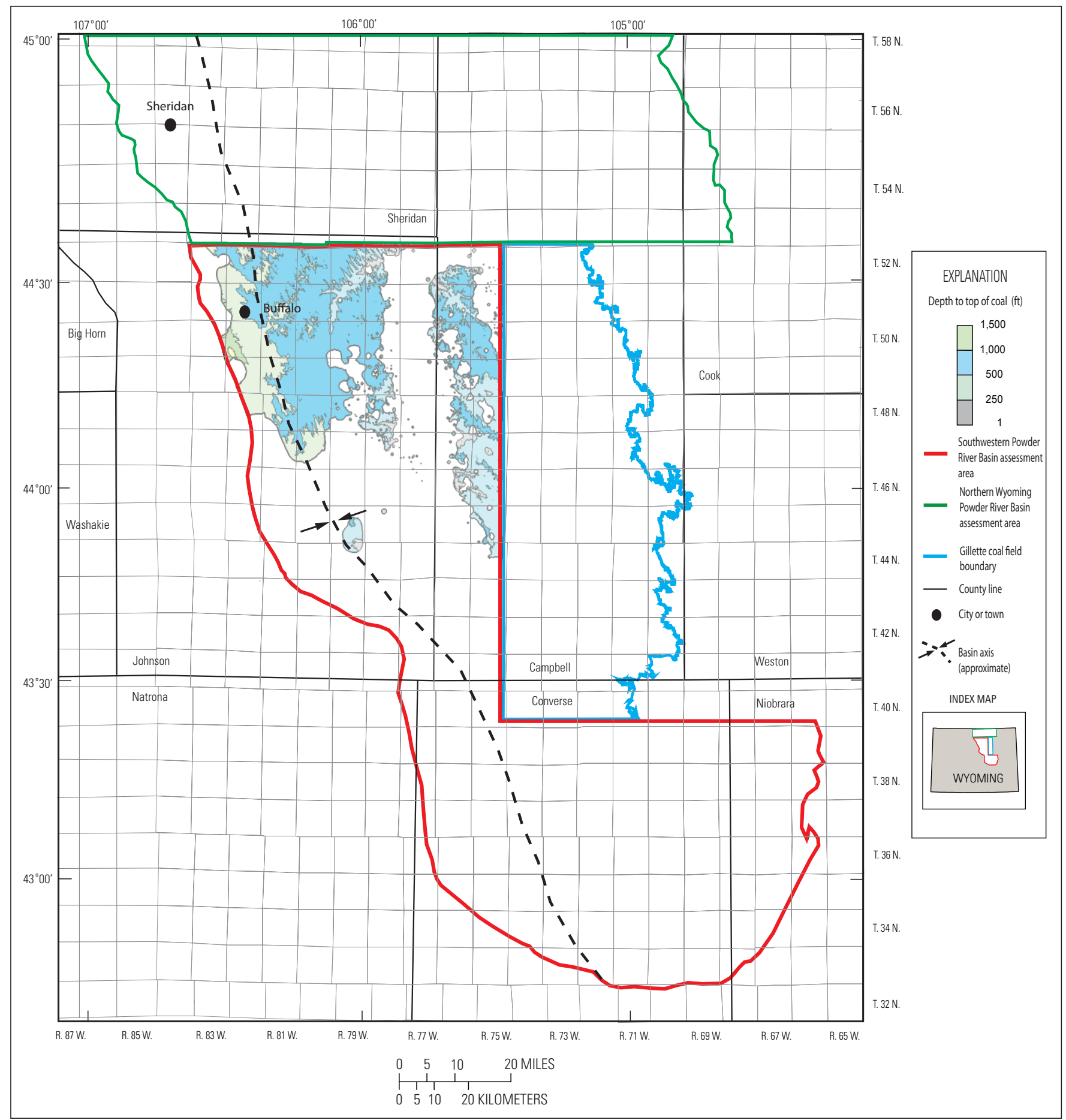

Figure 37. Depth to the top of the Lower Felix coal bed within the Southwestern Powder River Basin assessment area. 


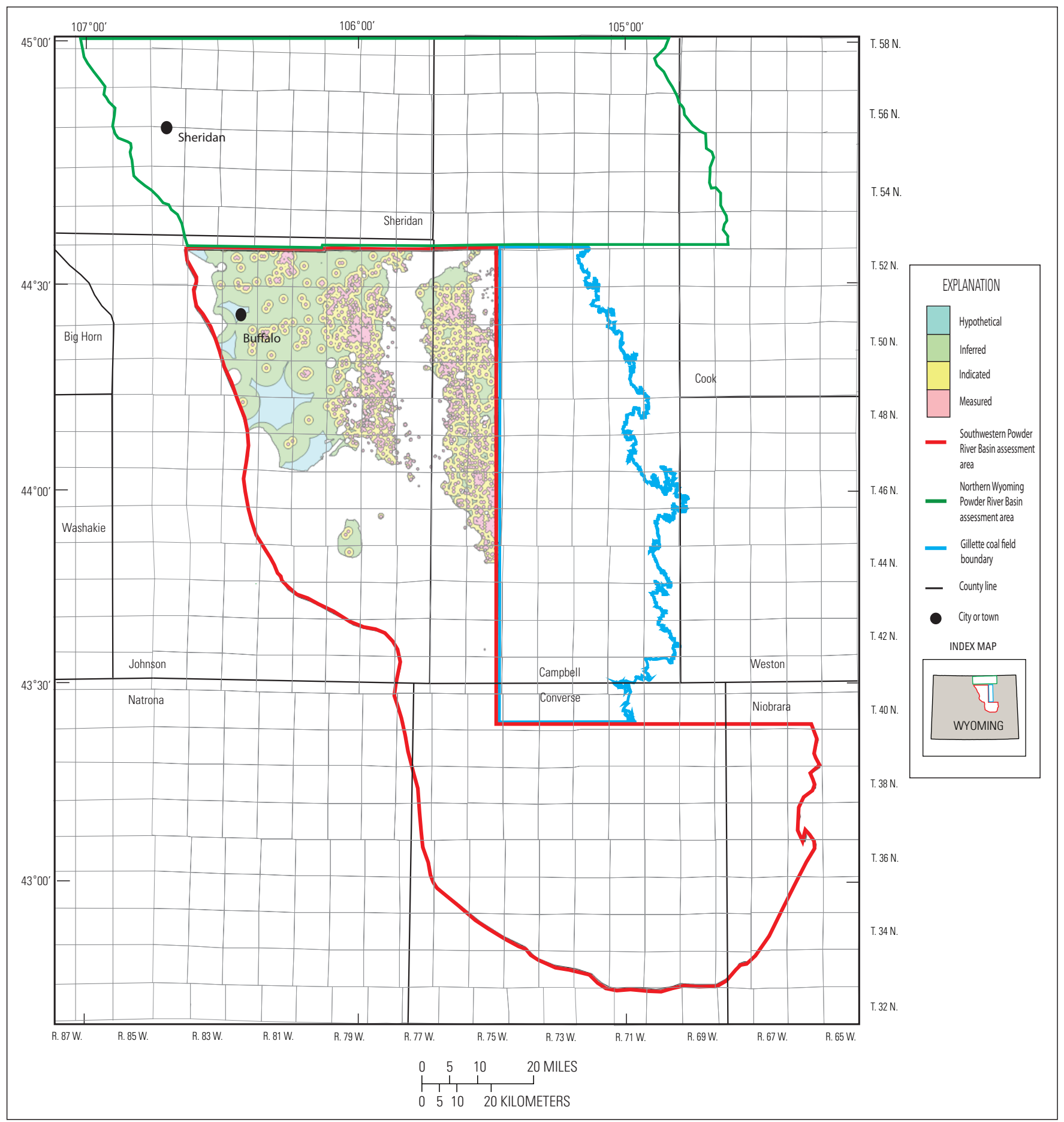

Figure 38. Coal resource reliability categories for the Lower Felix coal bed within the Southwestern Powder River Basin assessment area. 


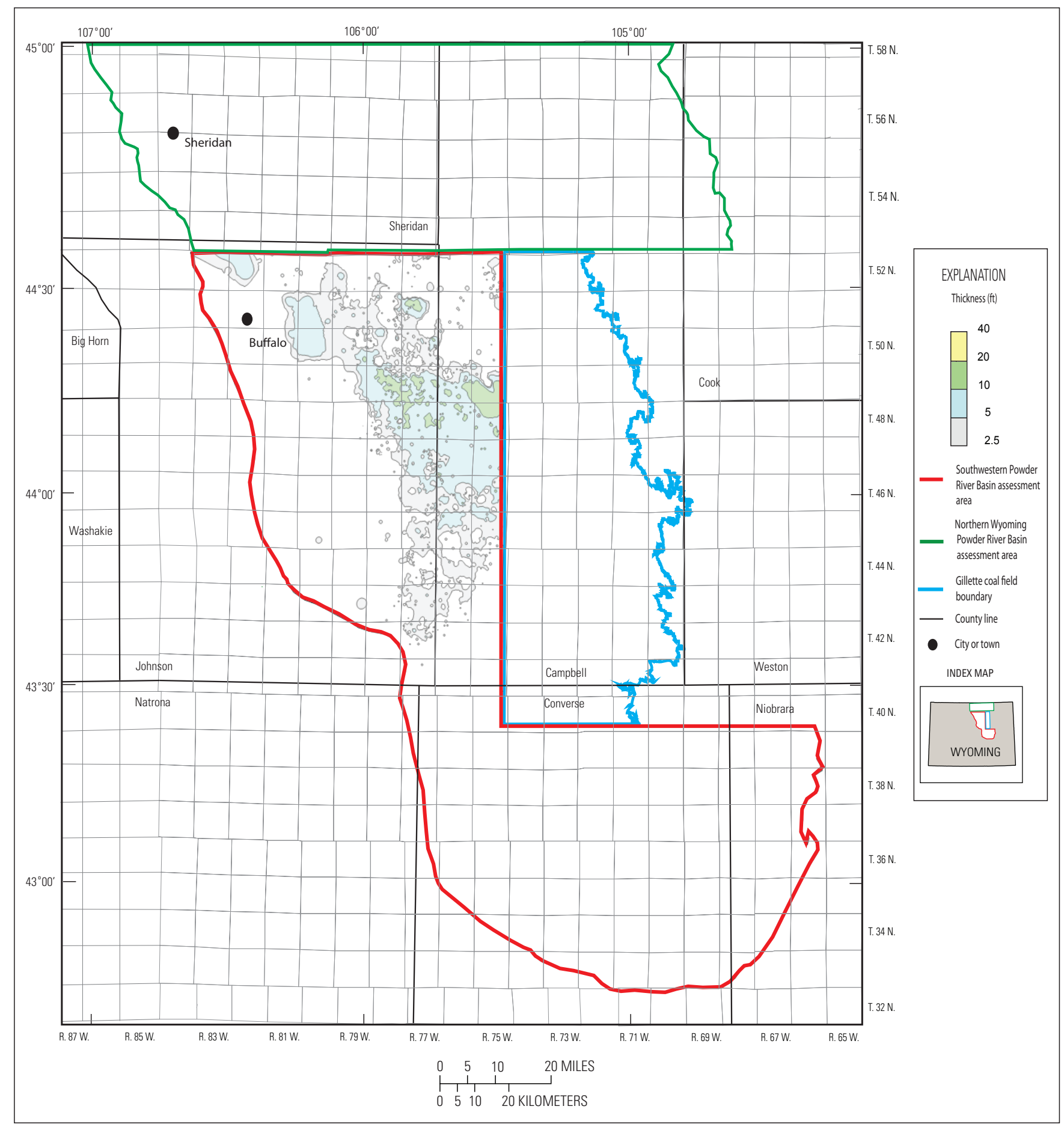

Figure 39. Isopach map of the Roland (Upper Rider) coal bed showing extent of resources greater than $2.5 \mathrm{ft}$ thick within the Southwestern Powder River Basin assessment area. 


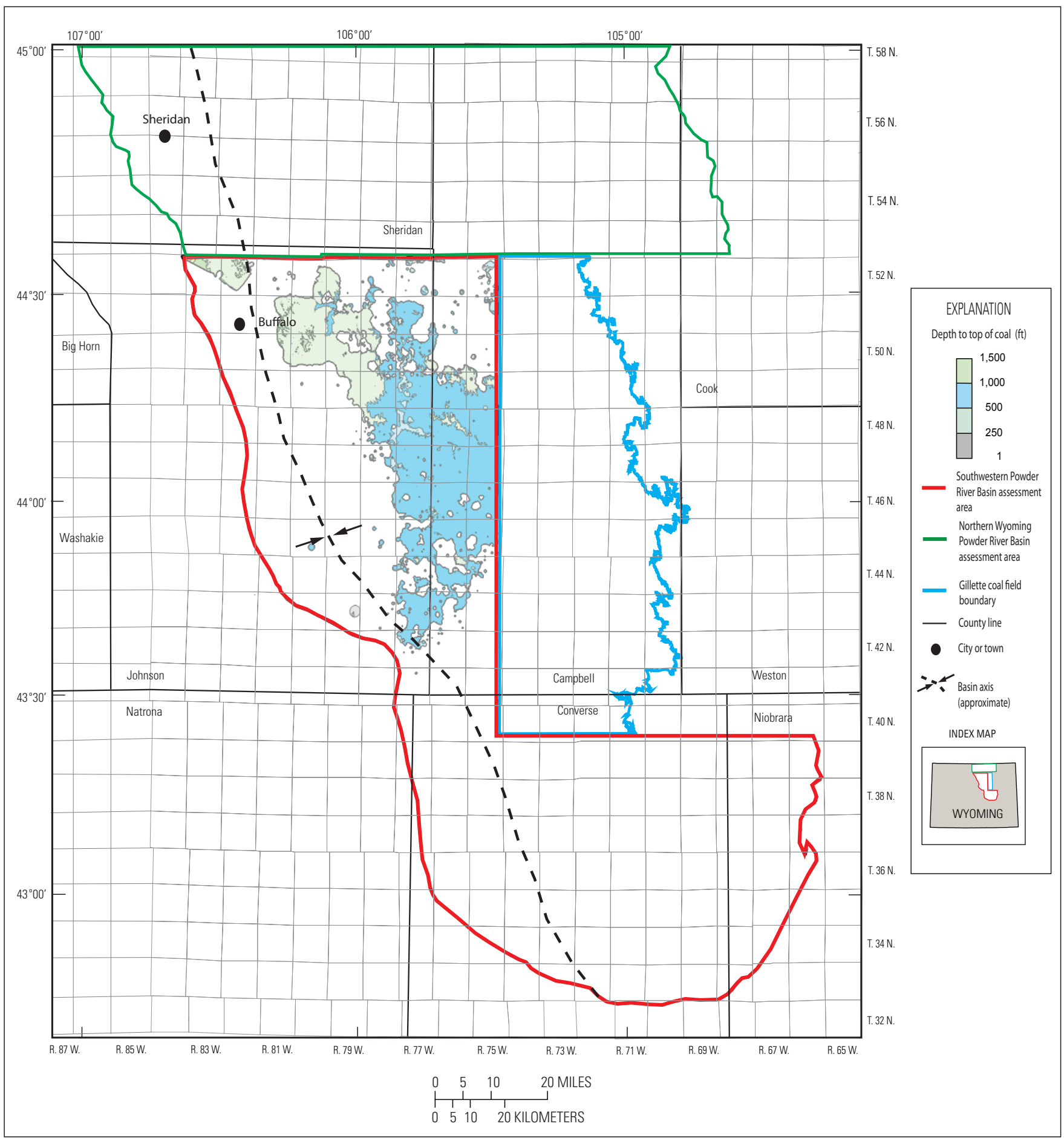

Figure 40. Depth to the top of the Roland (Upper Rider) coal bed within the Southwestern Powder River Basin assessment area. 


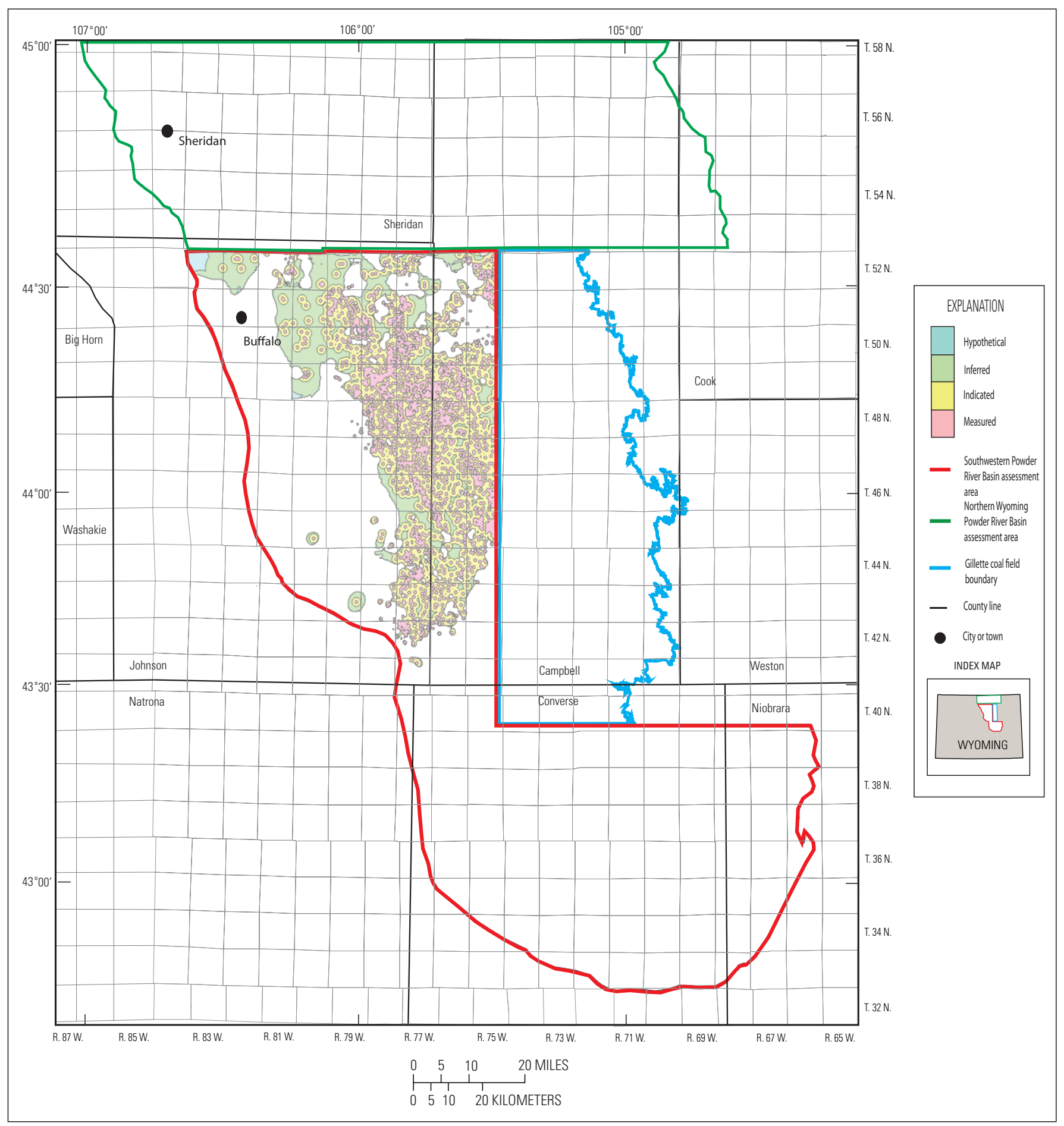

Figure 41. Coal resource reliability categories for the Roland (Upper Rider) coal bed within the Southwestern Powder River Basin assessment area. 


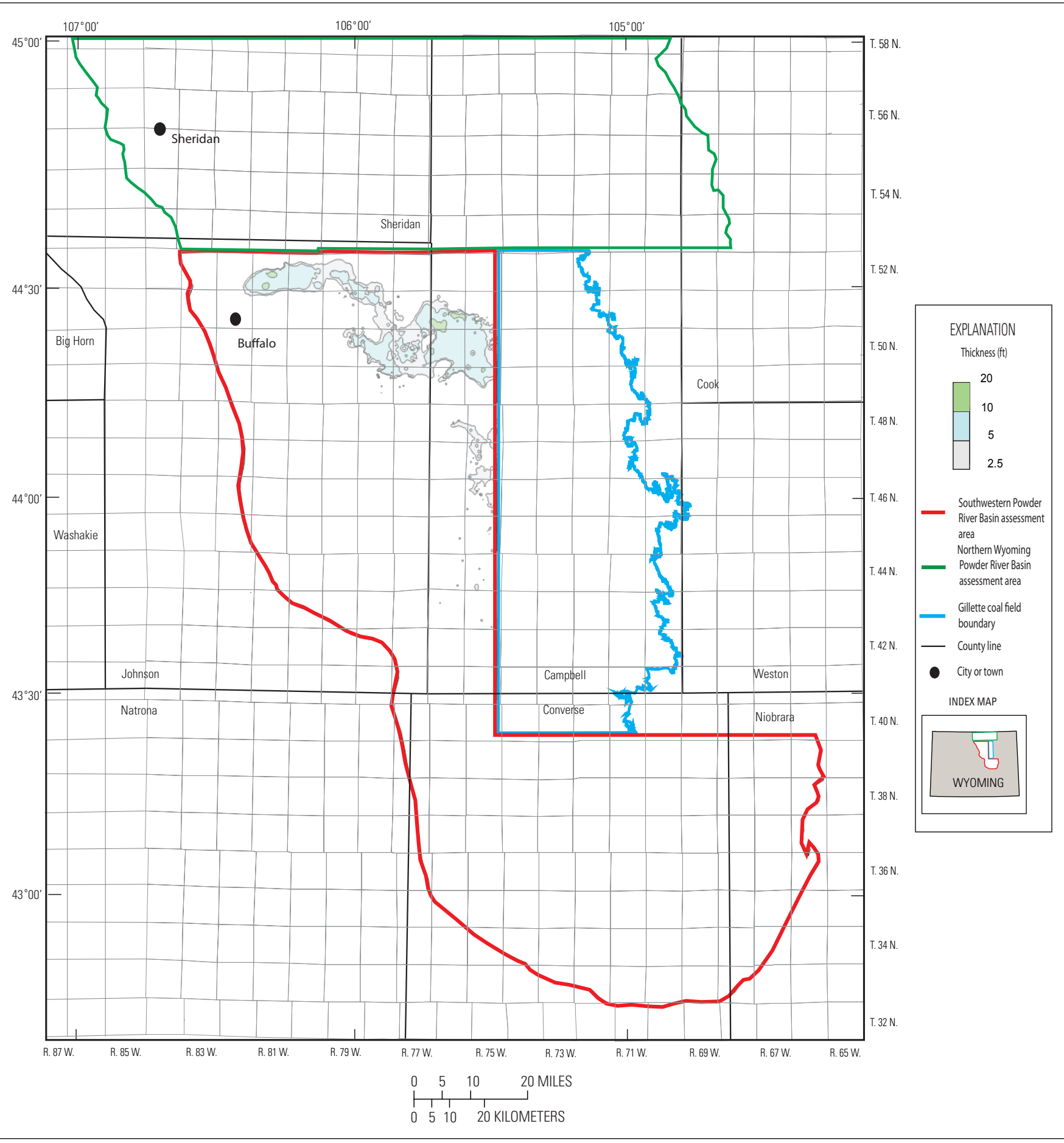

Figure 42. Isopach map of the Roland (Upper Rider) coal bed showing extent of resources greater than $2.5 \mathrm{ft}$ thick within the Southwestern Powder River Basin assessment area. 


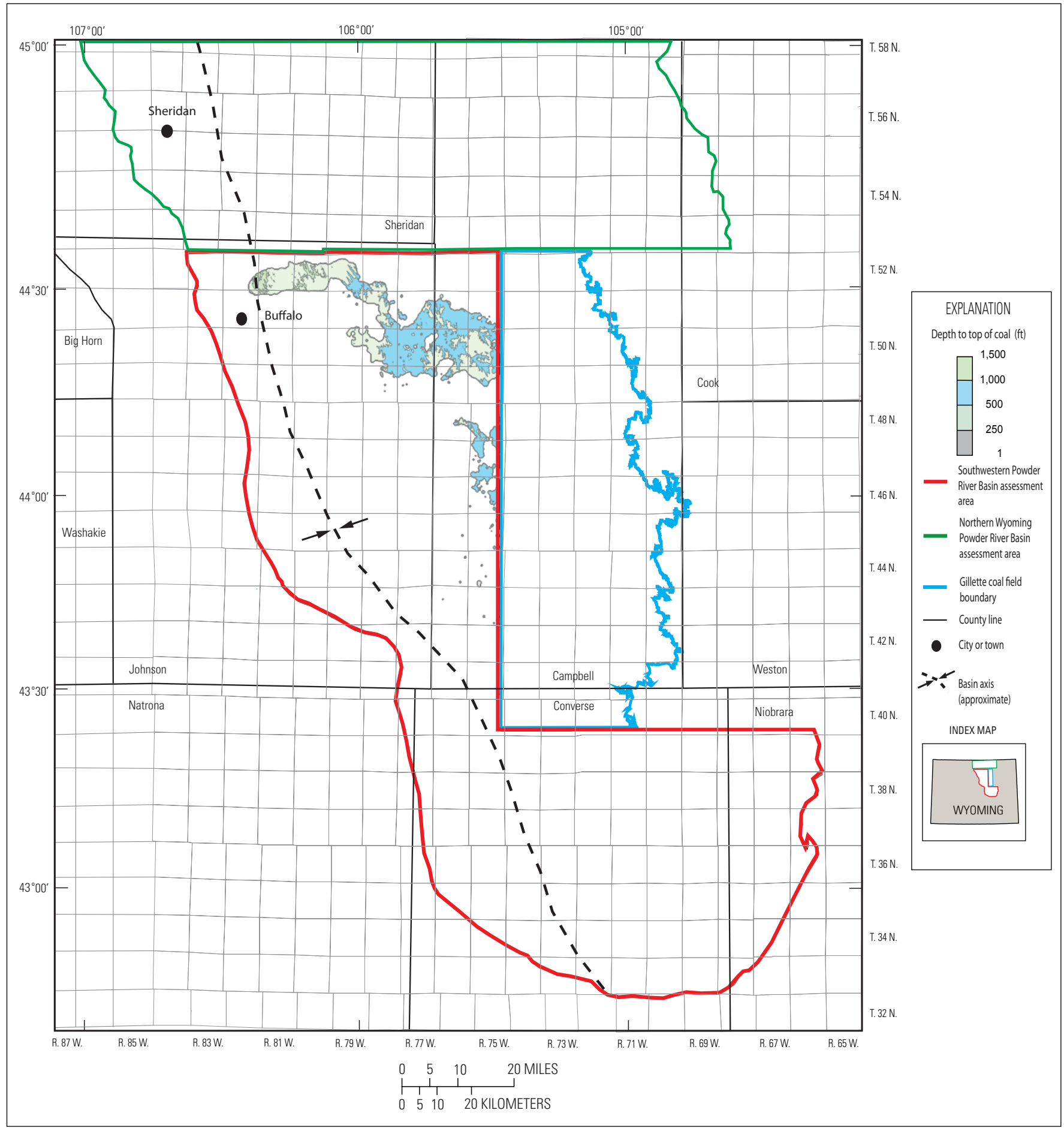

Figure 43. Depth to the top of the Roland (Lower Rider) coal bed within the Southwestern Powder River Basin assessment area. 


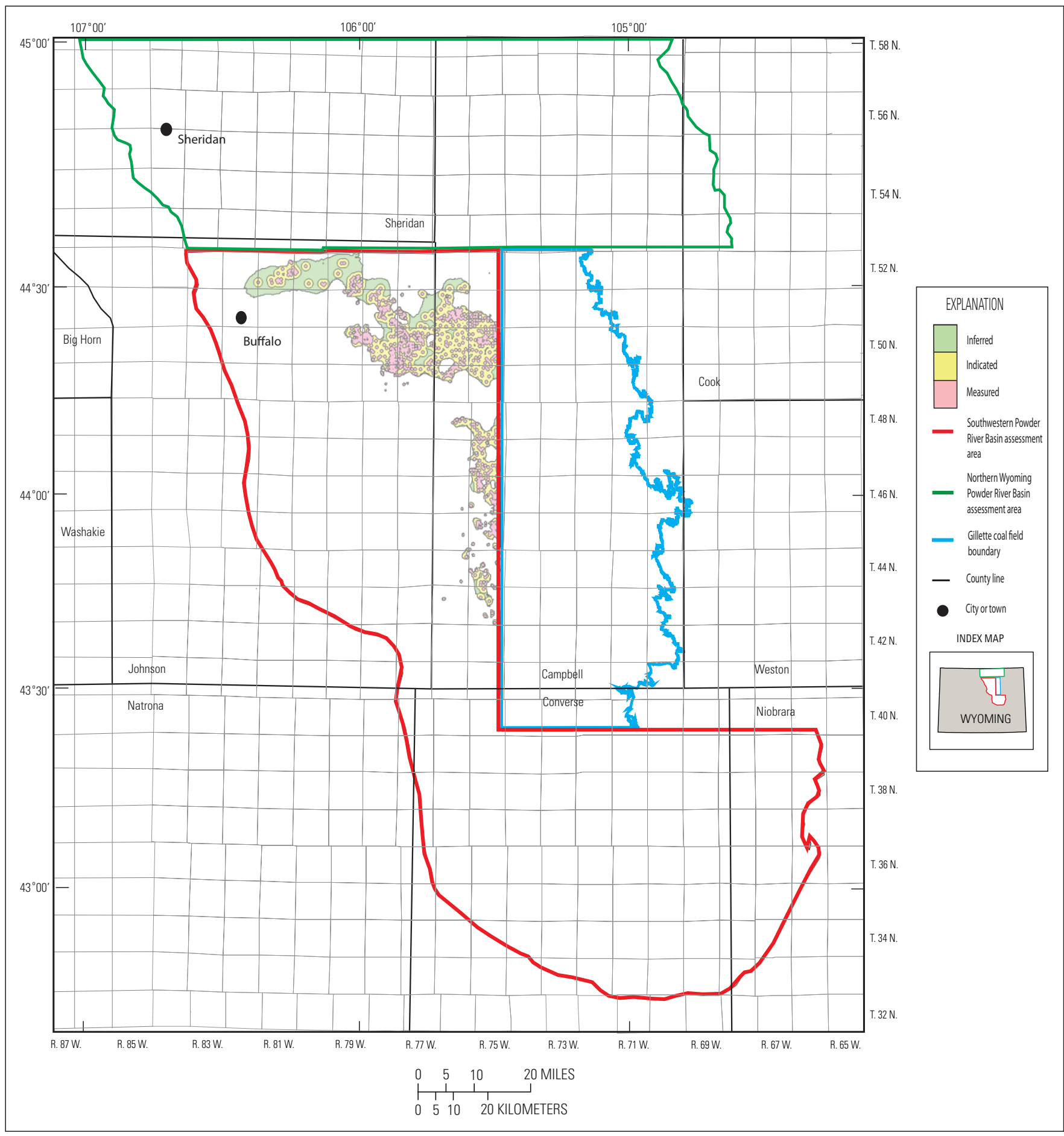

Figure 44. Coal resource reliability categories for the Roland (Lower Rider) coal bed within the Southwestern Powder River Basin assessment area. 


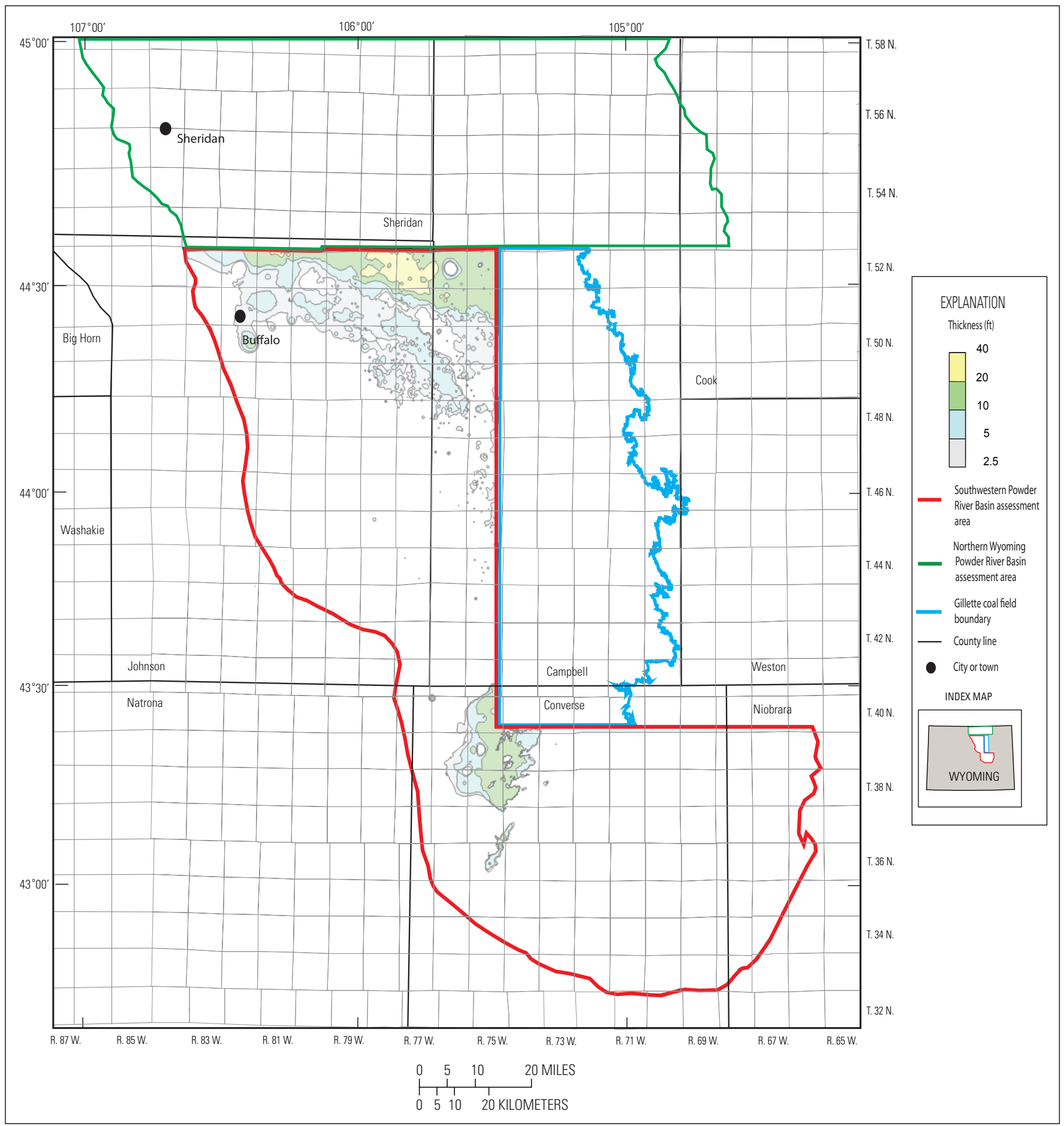

Figure 45. Isopach map of the Roland Baker (1929) coal bed showing extent of resources greater than $2.5 \mathrm{ft}$ thick within the Southwestern Powder River Basin assessment area. 


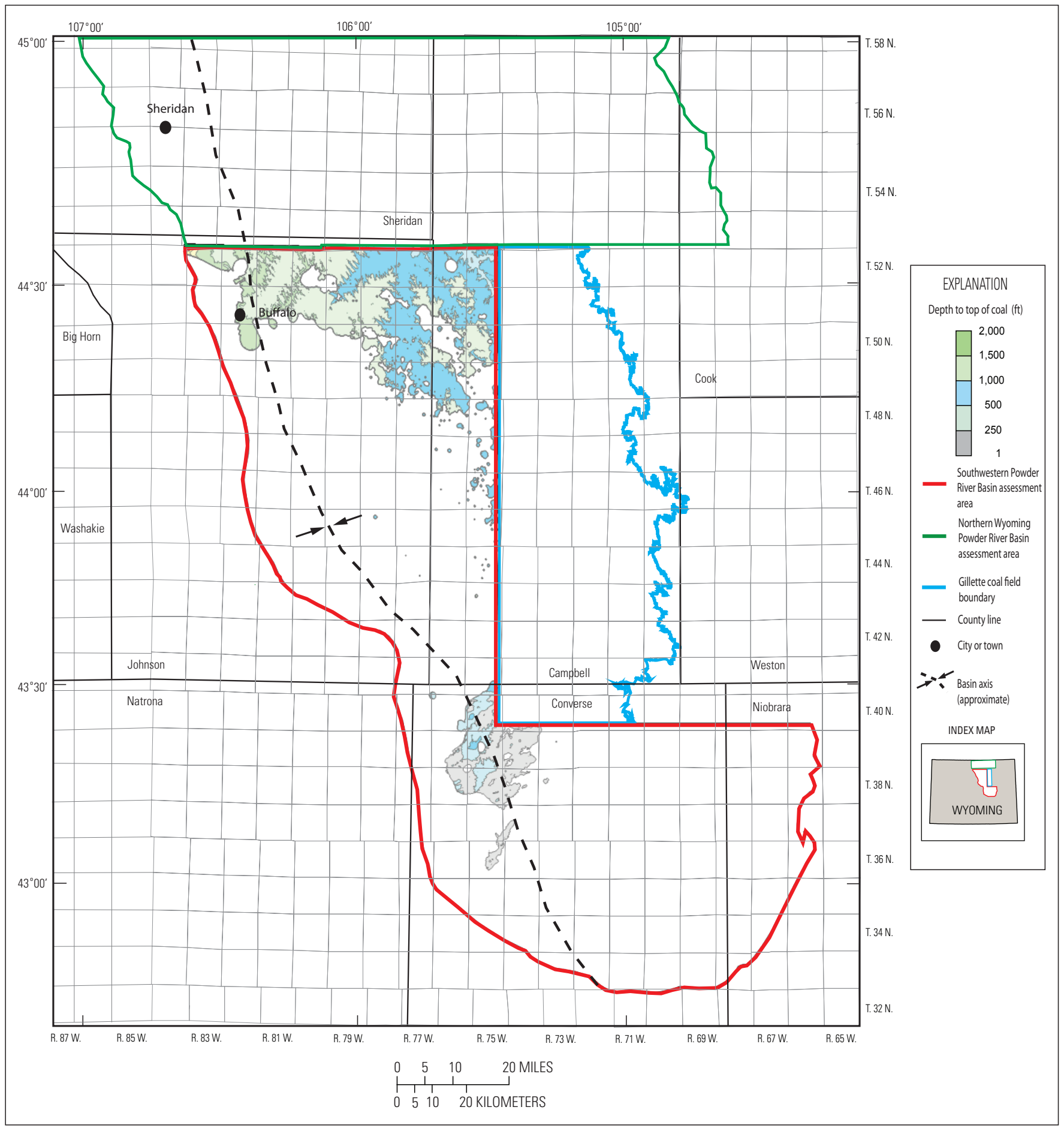

Figure 46. Depth to the top of the Roland Baker (1929) coal bed within the Southwestern Powder River Basin assessment area. 


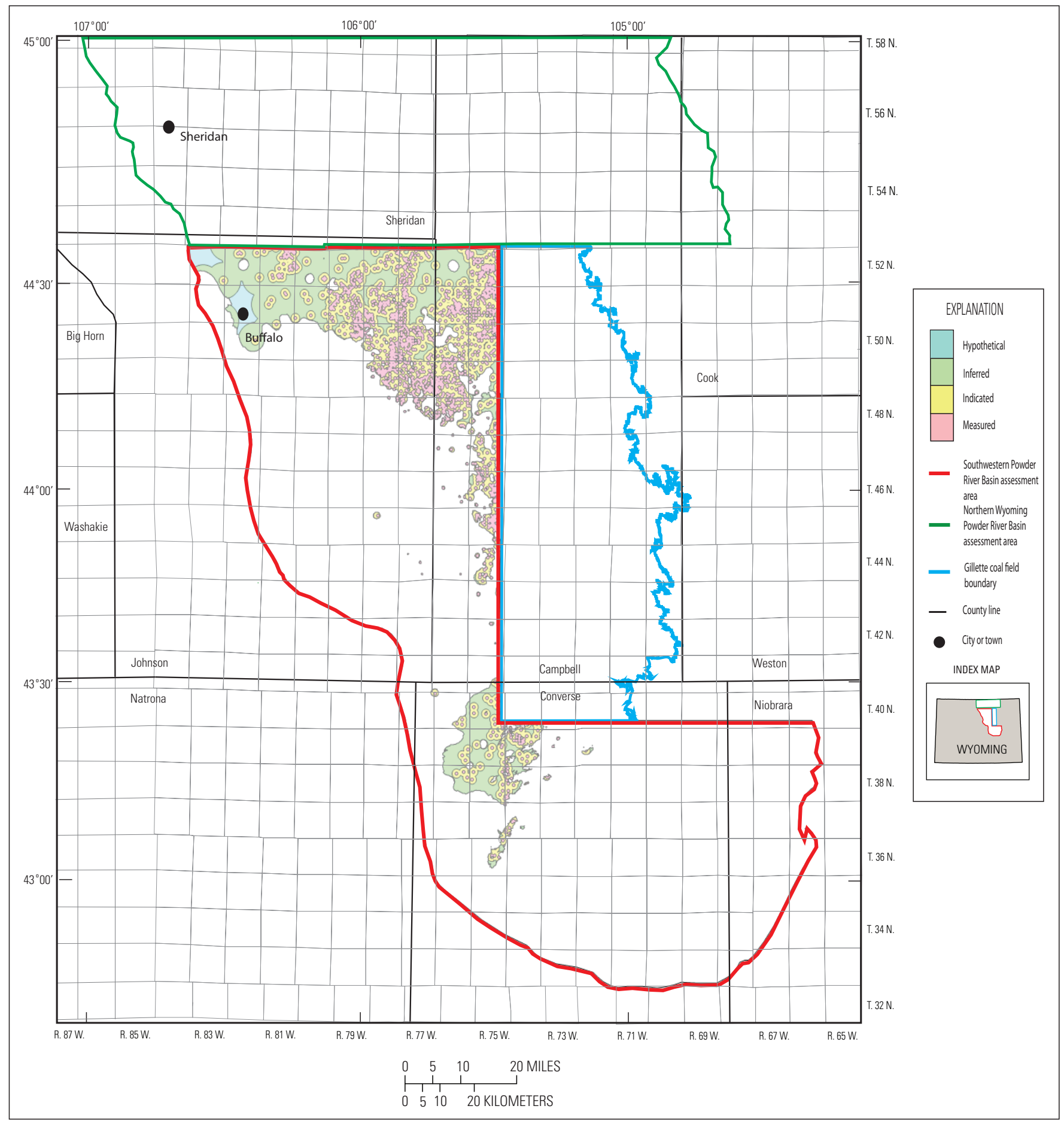

Figure 47. Coal resource reliability categories for the Roland Baker (1929) coal bed within the Southwestern Powder River Basin assessment area. 


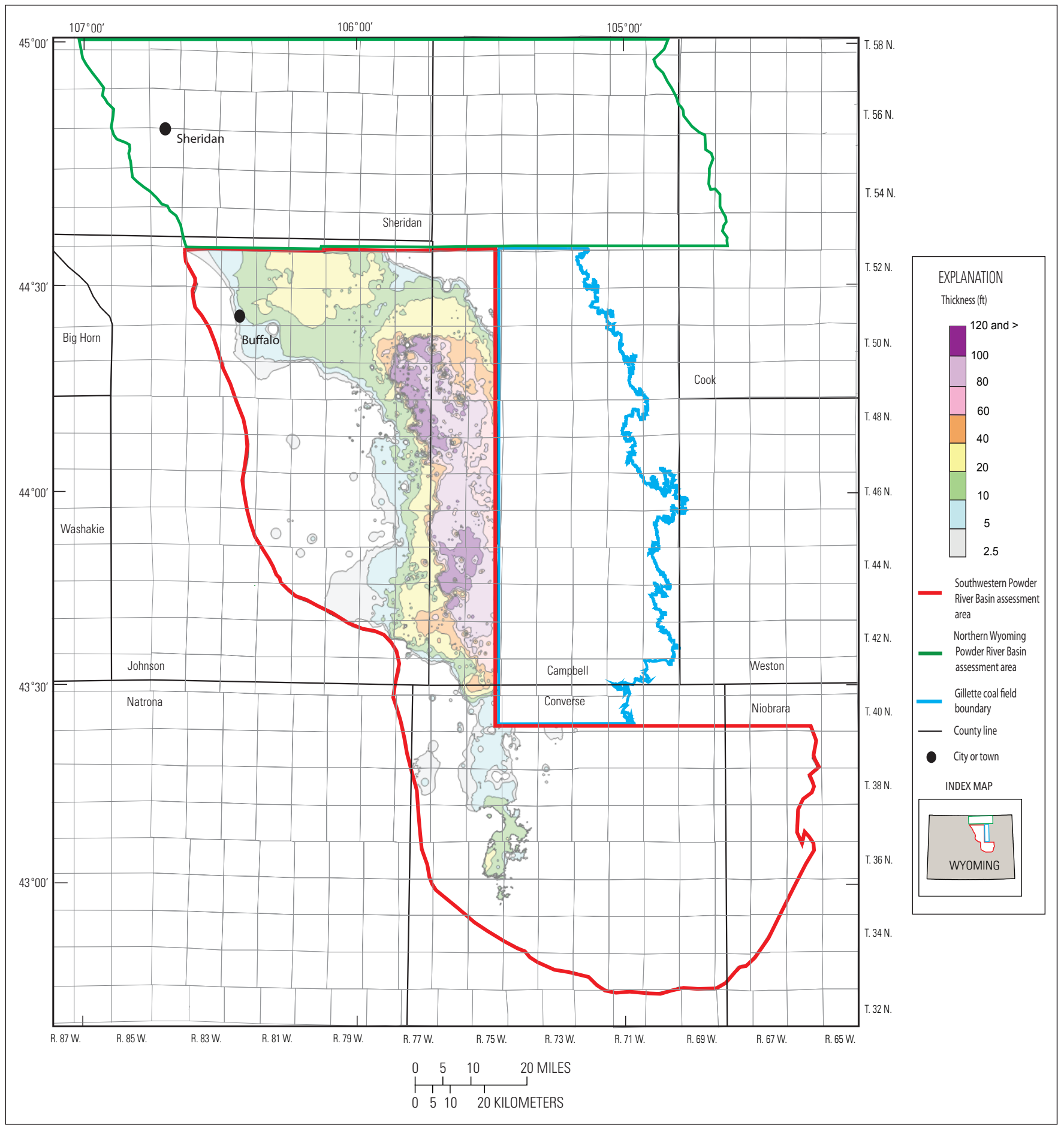

Figure 48. Isopach map of the Smith coal bed showing extent of resources greater than $2.5 \mathrm{ft}$ thick within the Southwestern Powder River Basin assessment area. 


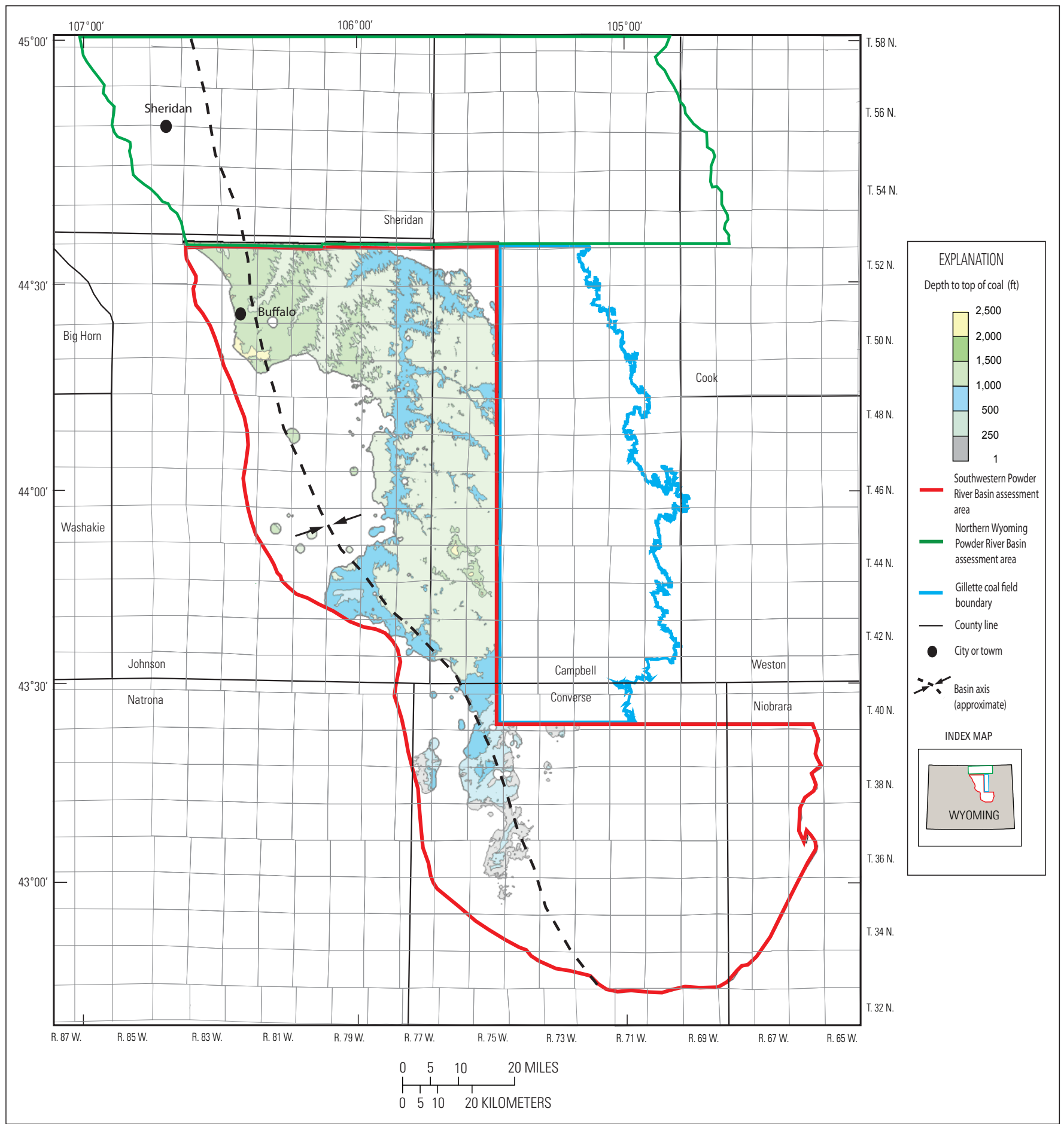

Figure 49. Depth to the top of the Smith coal bed within the Southwestern Powder River Basin assessment area. 


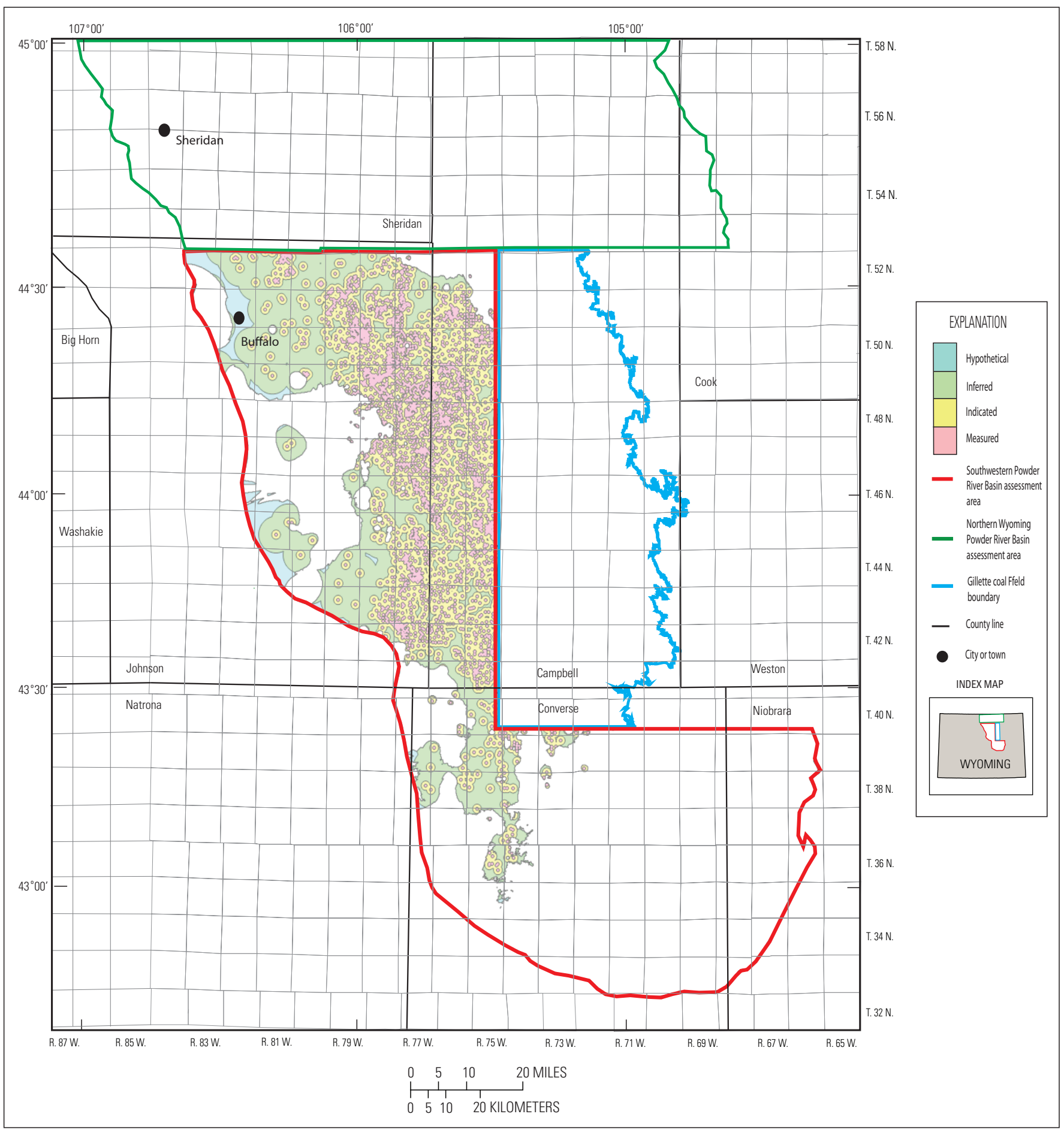

Figure 50. Coal resource reliability categories for the Smith coal bed within the Southwestern Powder River Basin assessment area. 


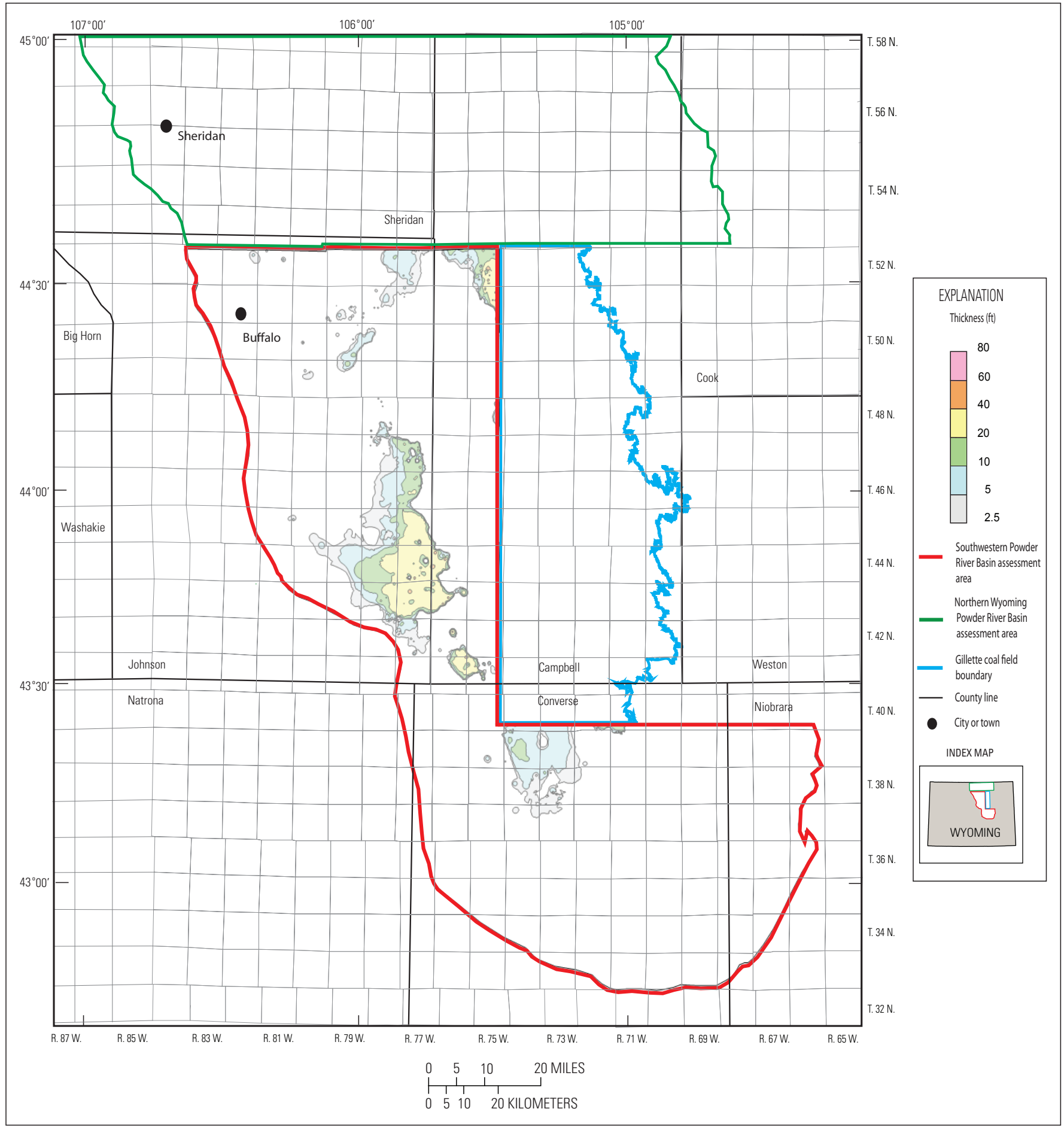

Figure 51. Isopach map of the Anderson coal bed showing extent of resources greater than $2.5 \mathrm{ft}$ thick within the Southwestern Powder River Basin assessment area. 


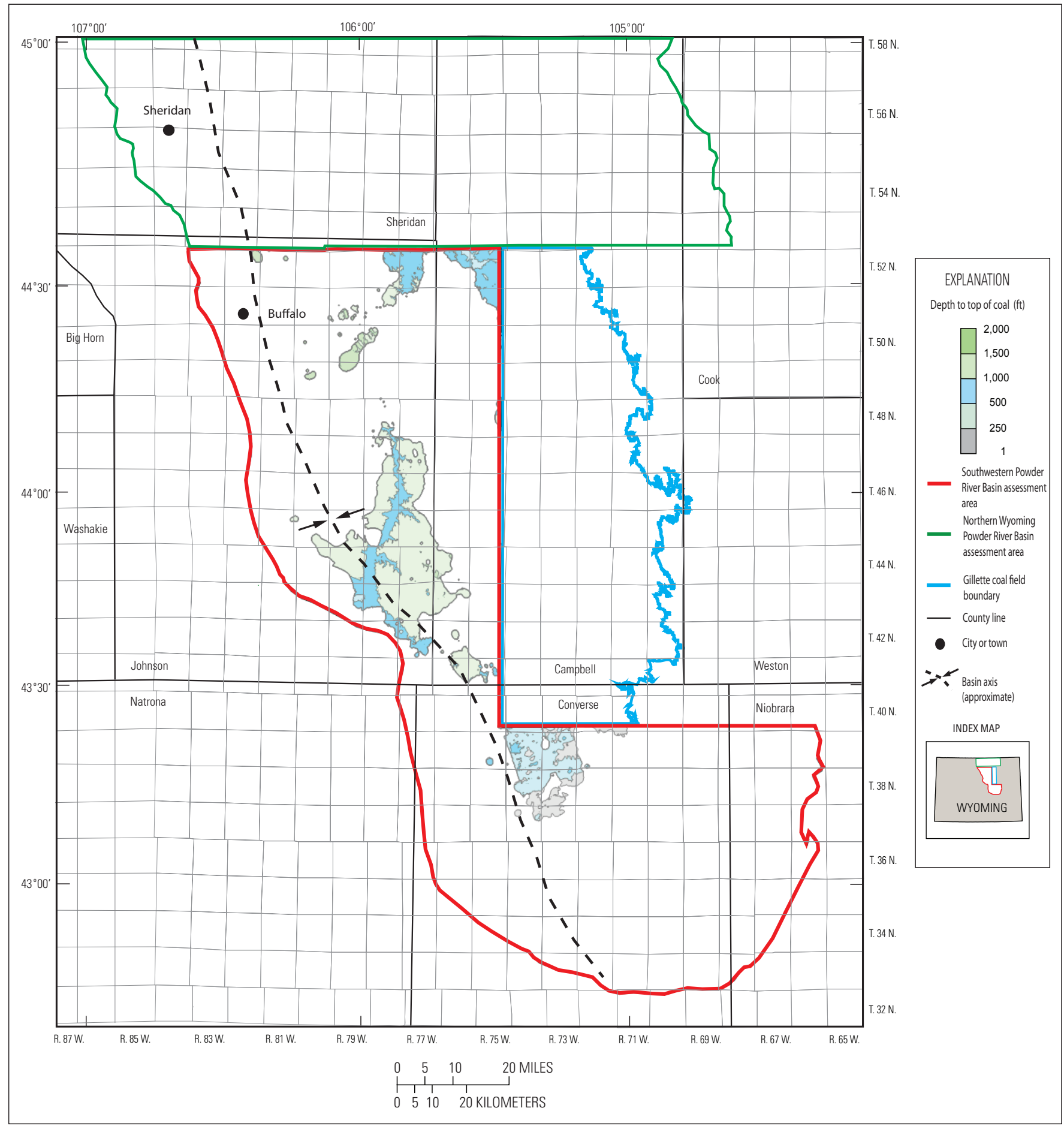

Figure 52. Depth to the top of the Anderson coal bed within the Southwestern Powder River Basin assessment area. 


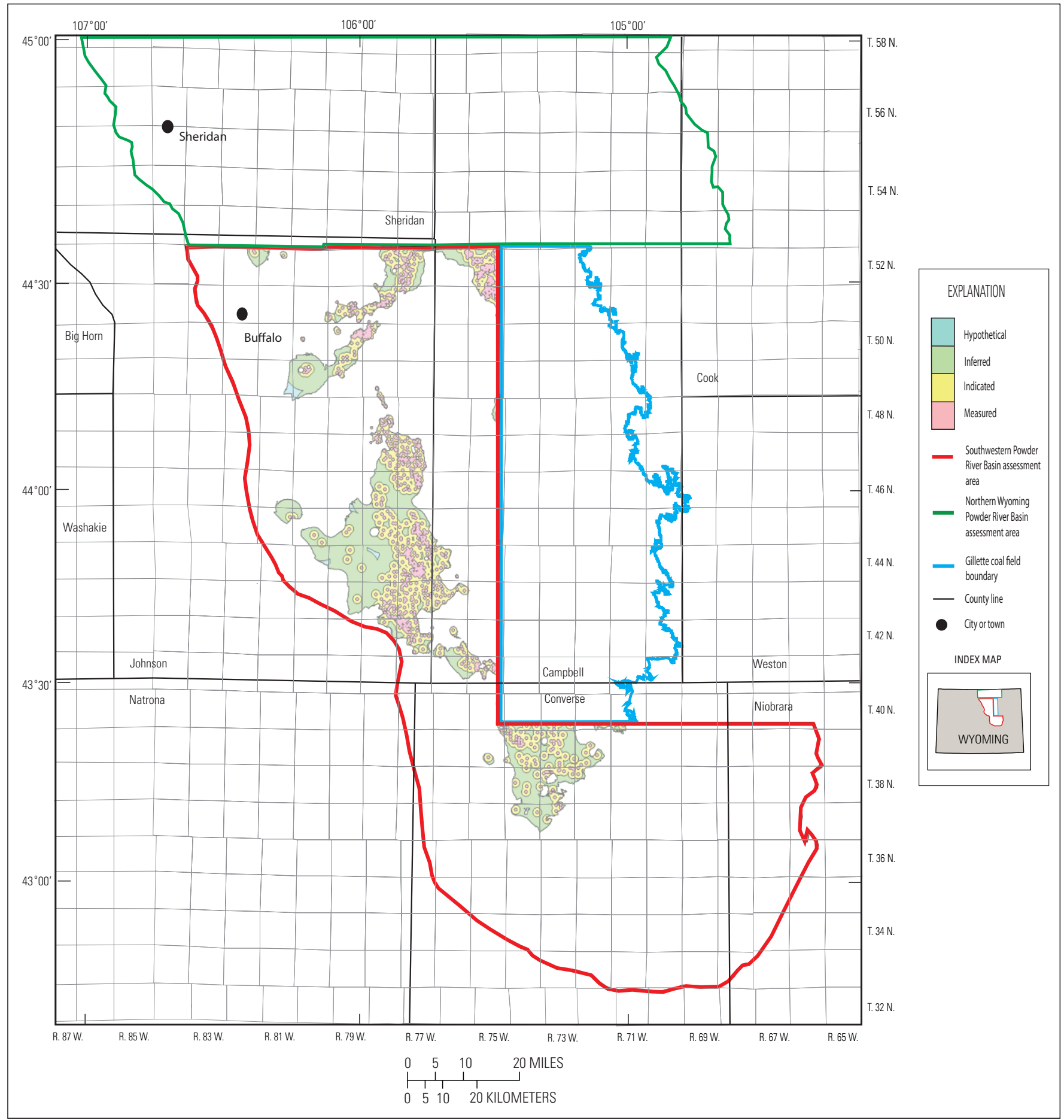

Figure 53. Coal resource reliability categories for the Anderson coal bed within the Southwestern Powder River Basin assessment area. 


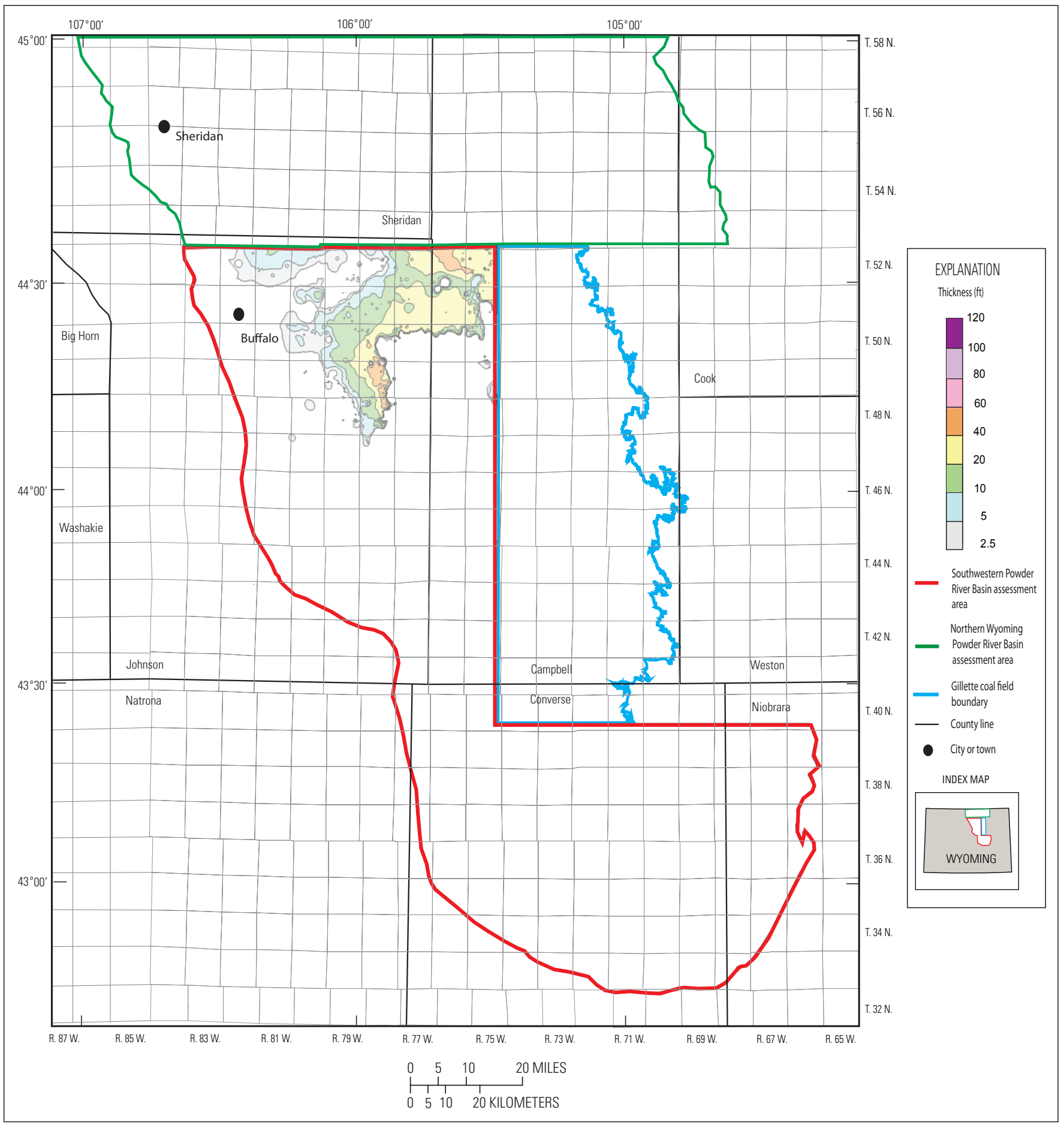

Figure 54. Isopach map of the Dietz 3 coal bed showing extent of resources greater than $2.5 \mathrm{ft}$ thick within the Southwestern Powder River Basin assessment area. 


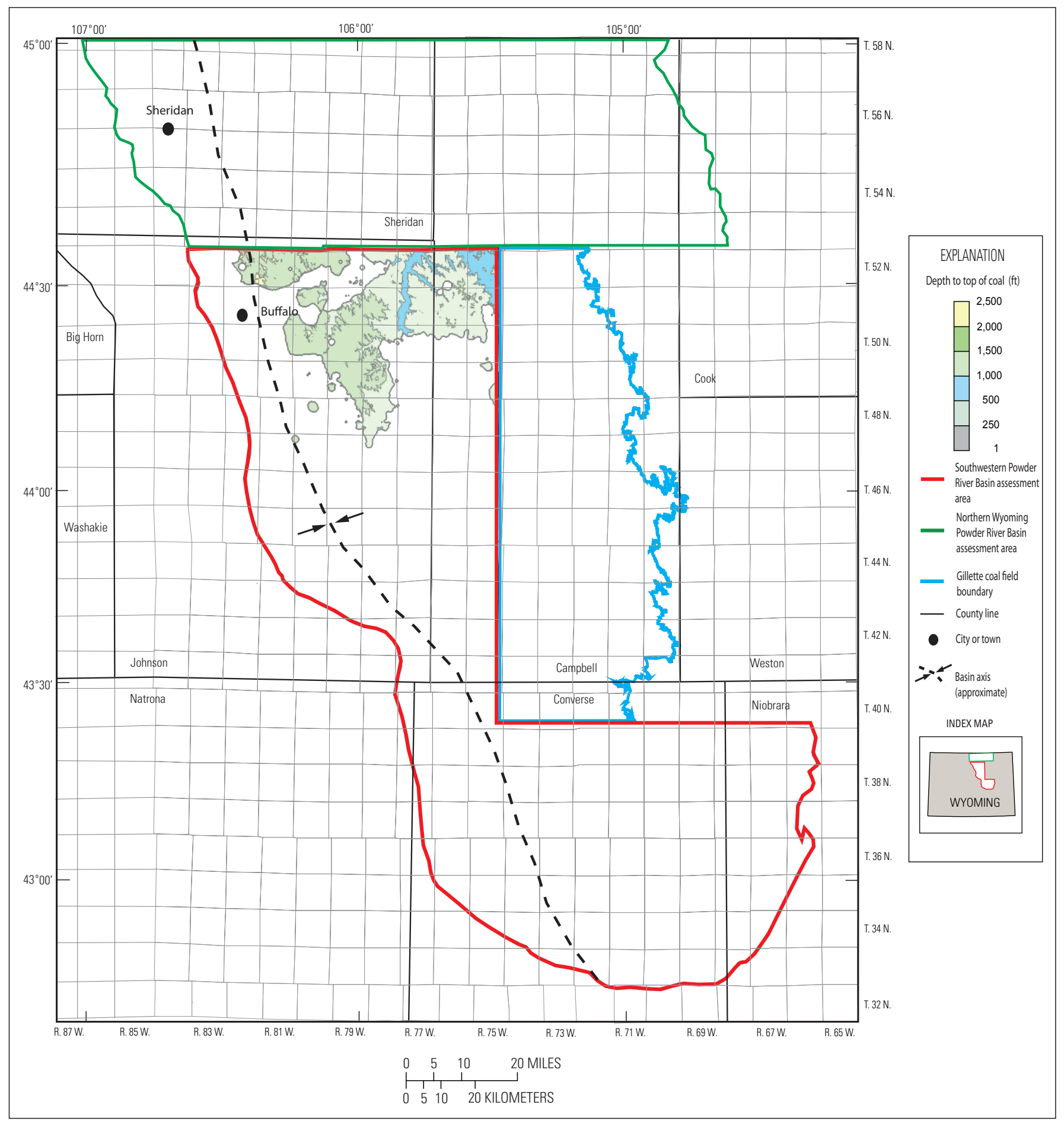

Figure 55. Depth to the top of the Dietz 3 coal bed within the Southwestern Powder River Basin assessment area. 


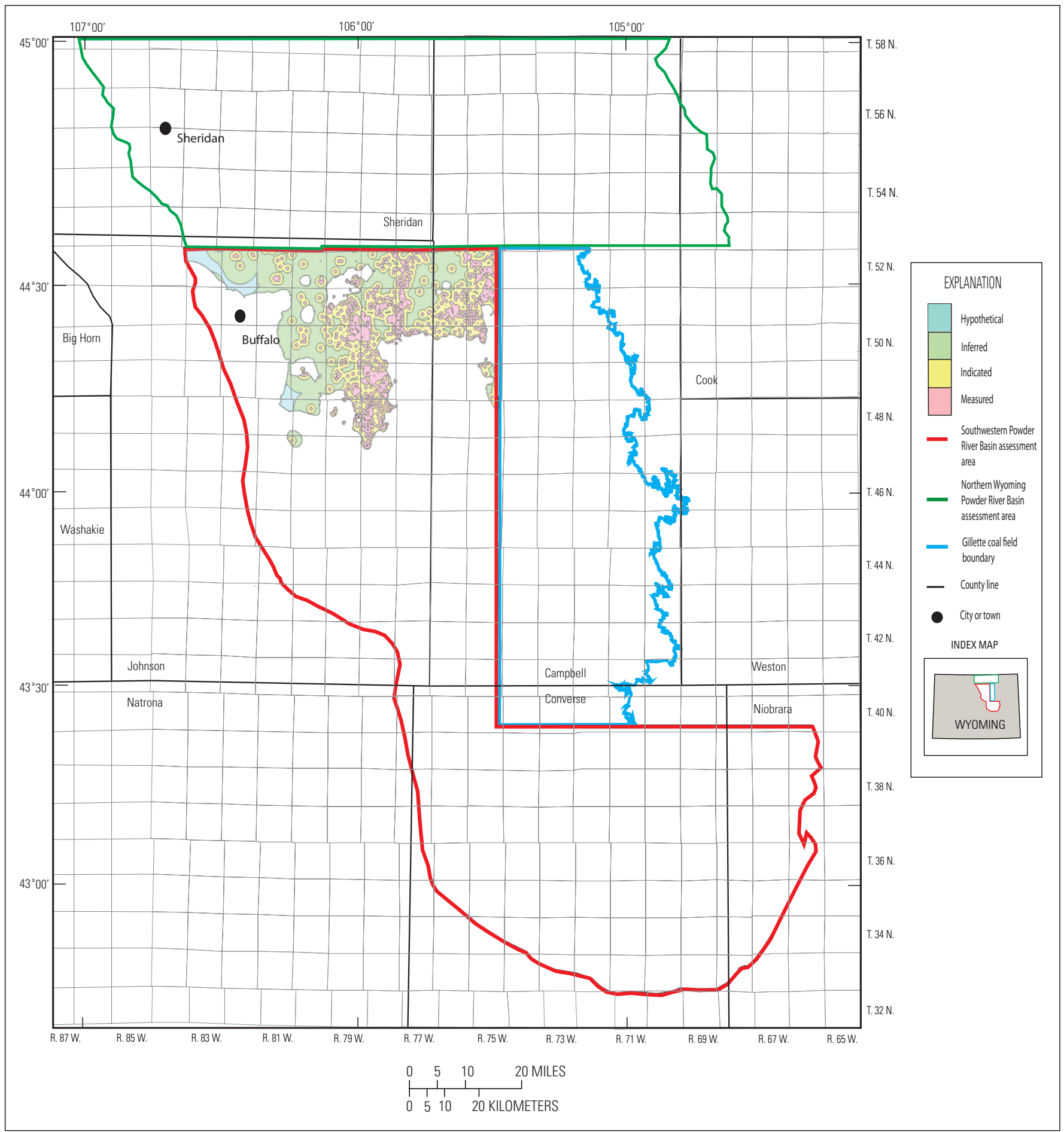

Figure 56. Coal resource reliability categories for the Dietz 3 coal bed within the Southwestern Powder River Basin assessment area. 


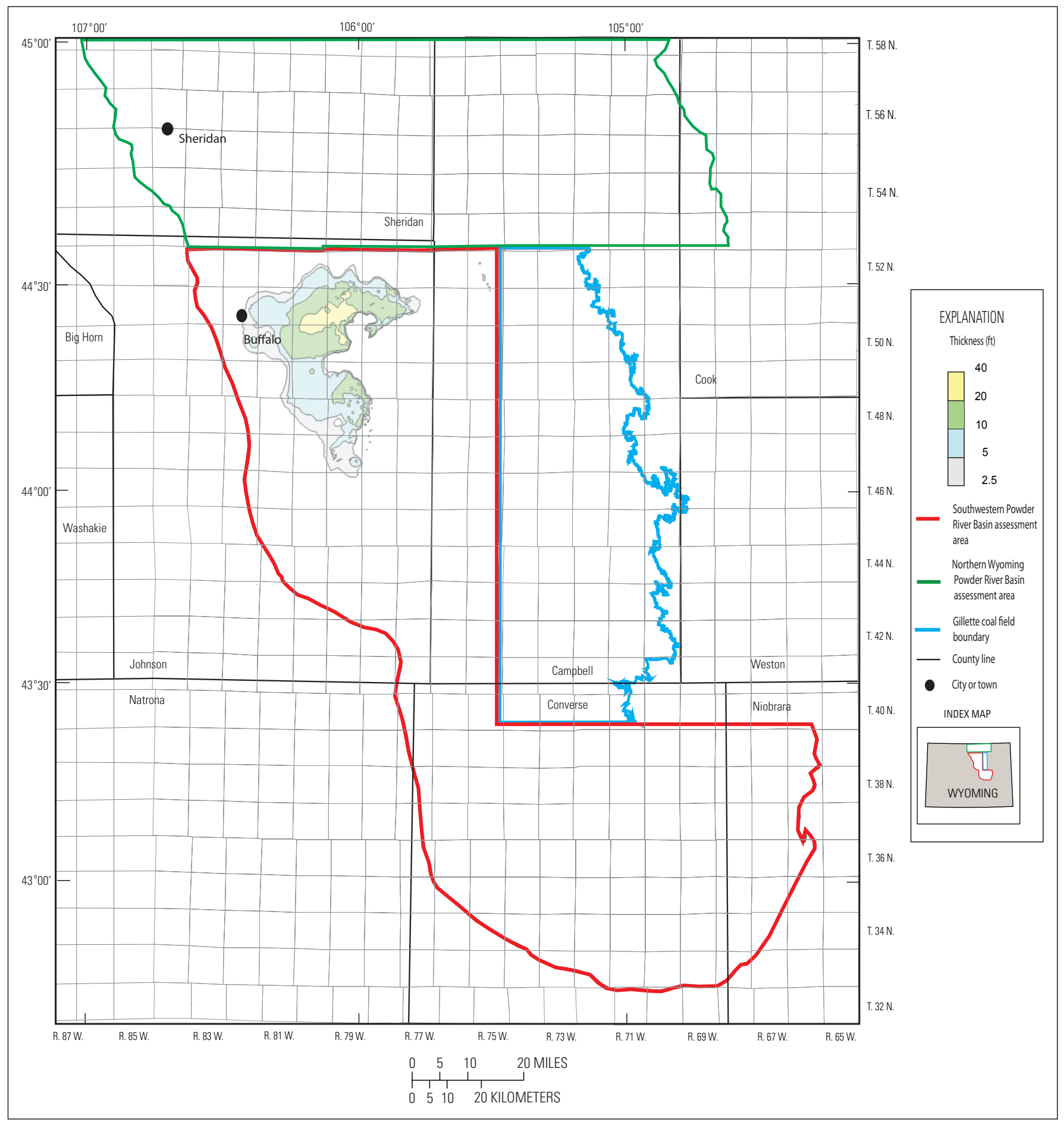

Figure 57. Isopach map of the Upper Canyon coal bed showing extent of resources greater than $2.5 \mathrm{ft}$ thick within the Southwestern Powder River Basin assessment area. 


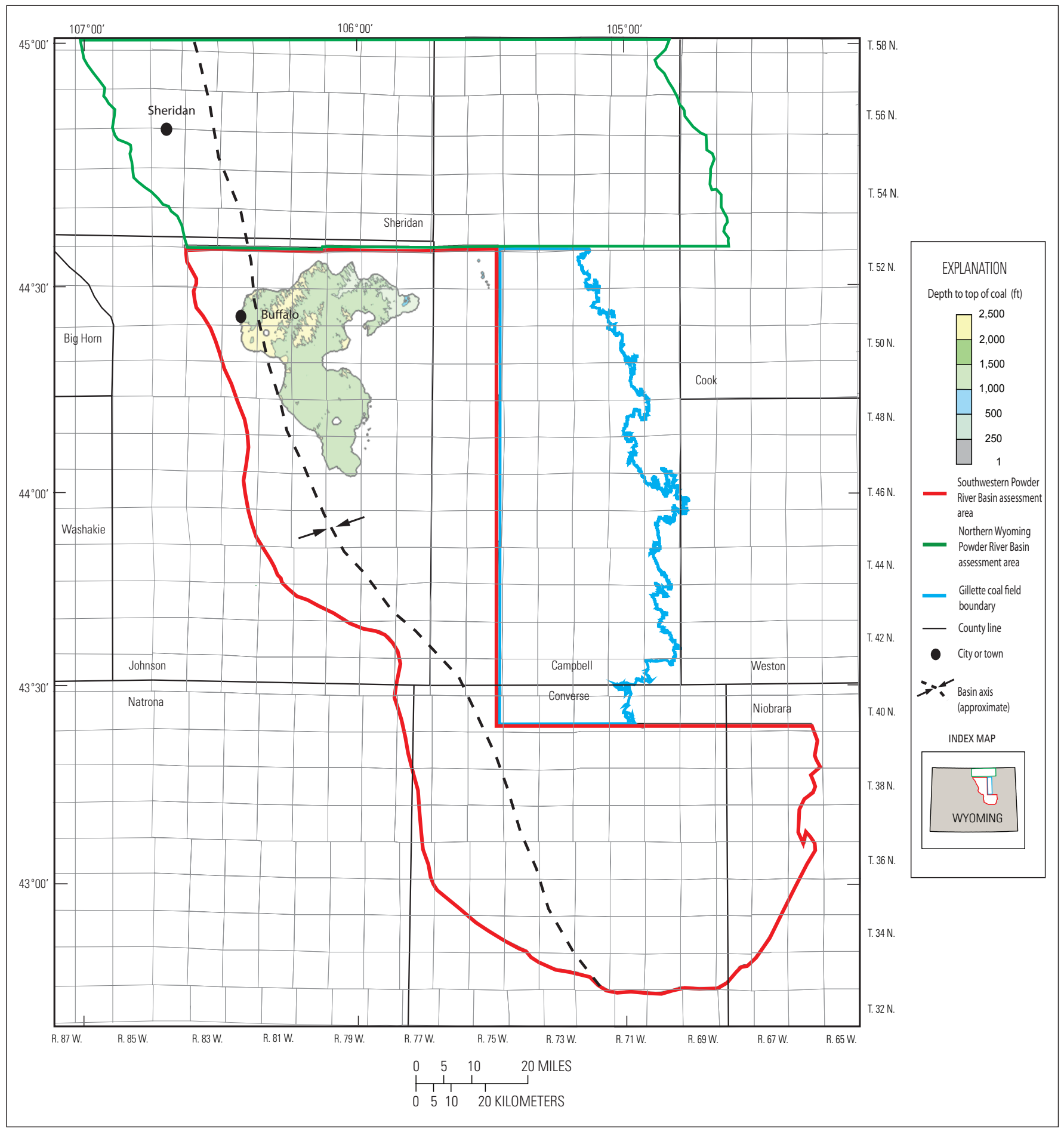

Figure 58. Depth to the top of the Upper Canyon coal bed within the Southwestern Powder River Basin assessment area. 


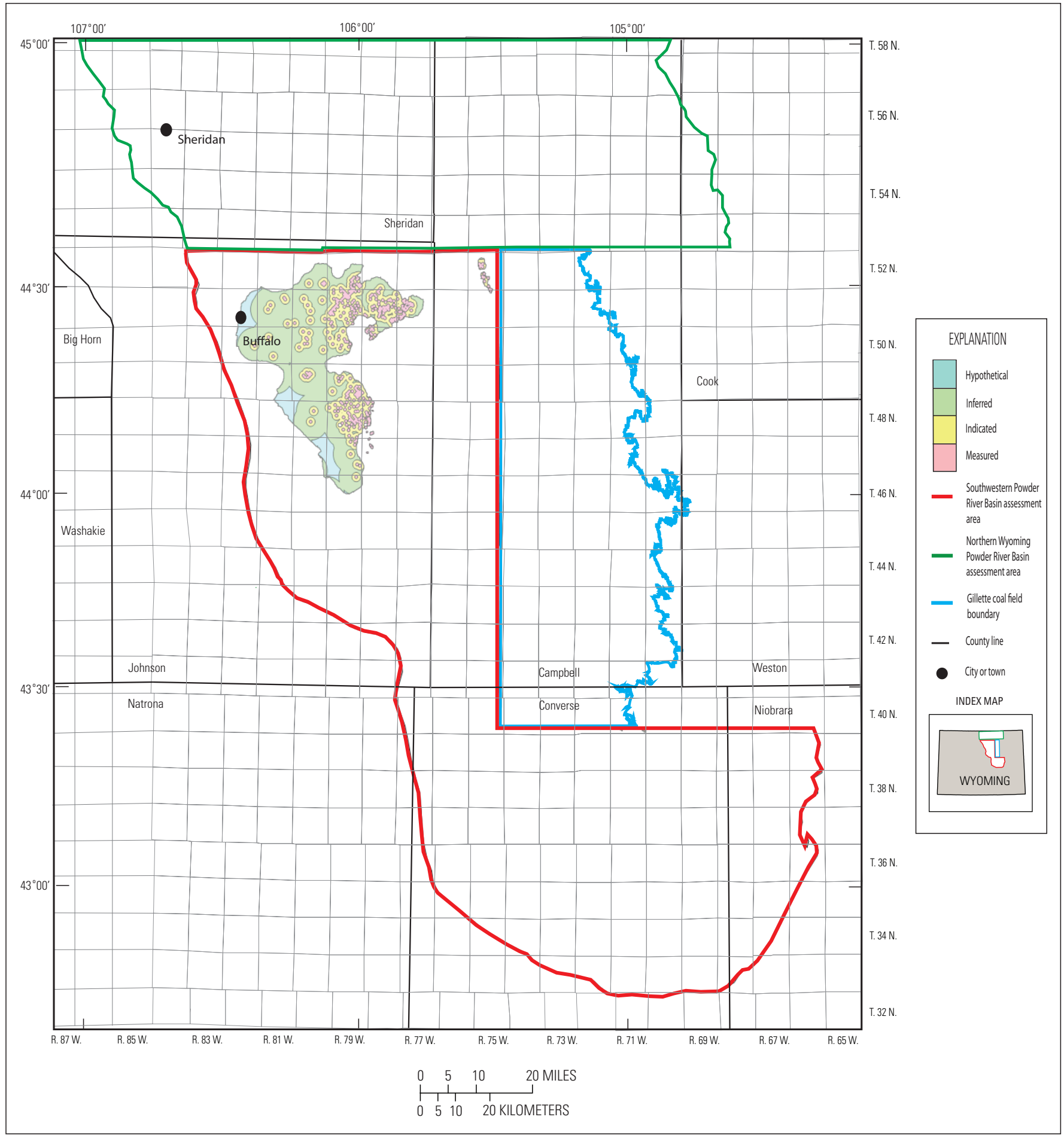

Figure 59. Coal resource reliability categories for the Upper Canyon coal bed within the Southwestern Powder River Basin assessment area. 


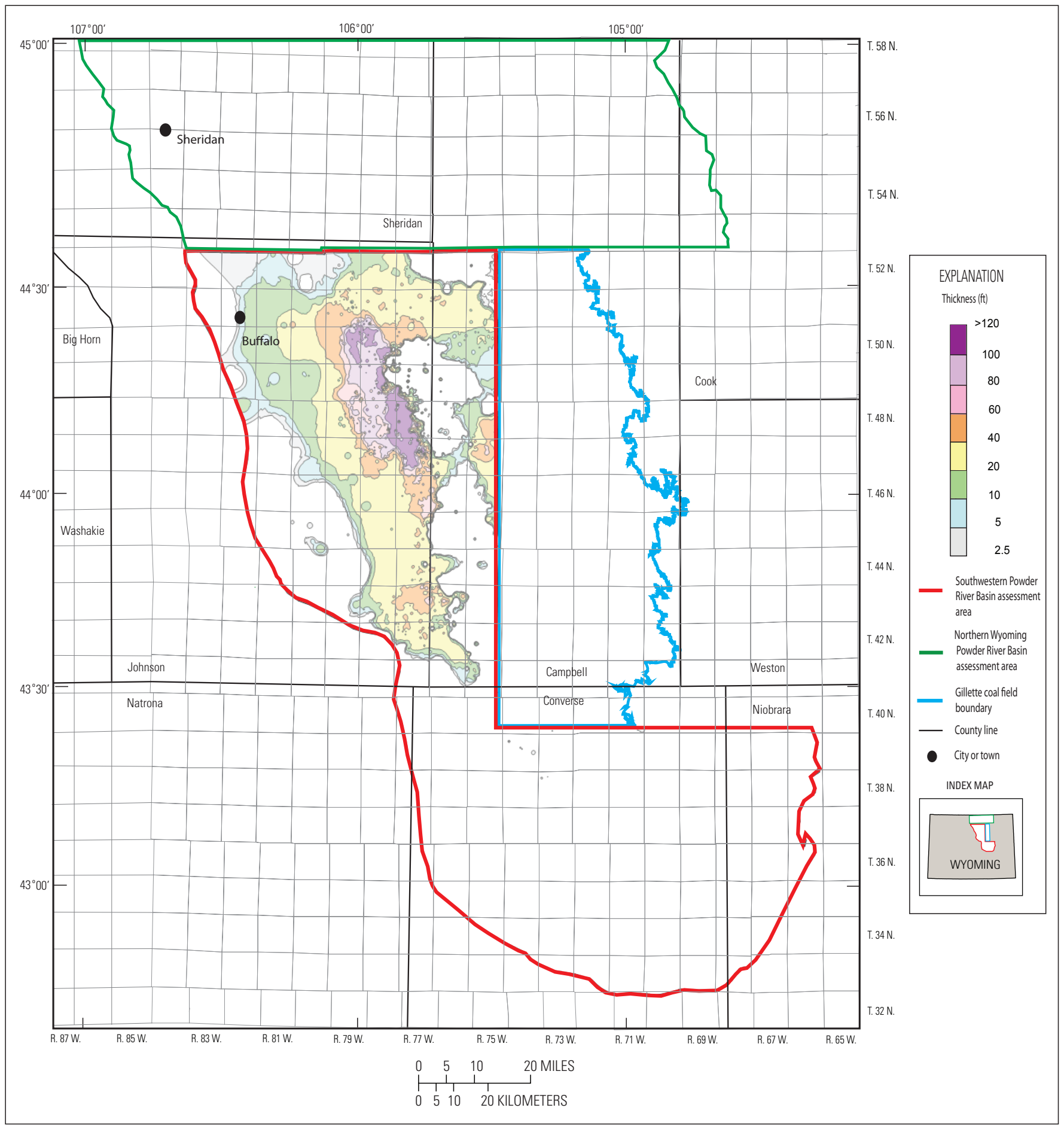

Figure 60. Isopach map of the Canyon coal bed showing extent of resources greater than $2.5 \mathrm{ft}$ thick within the Southwestern Powder River Basin assessment area. 


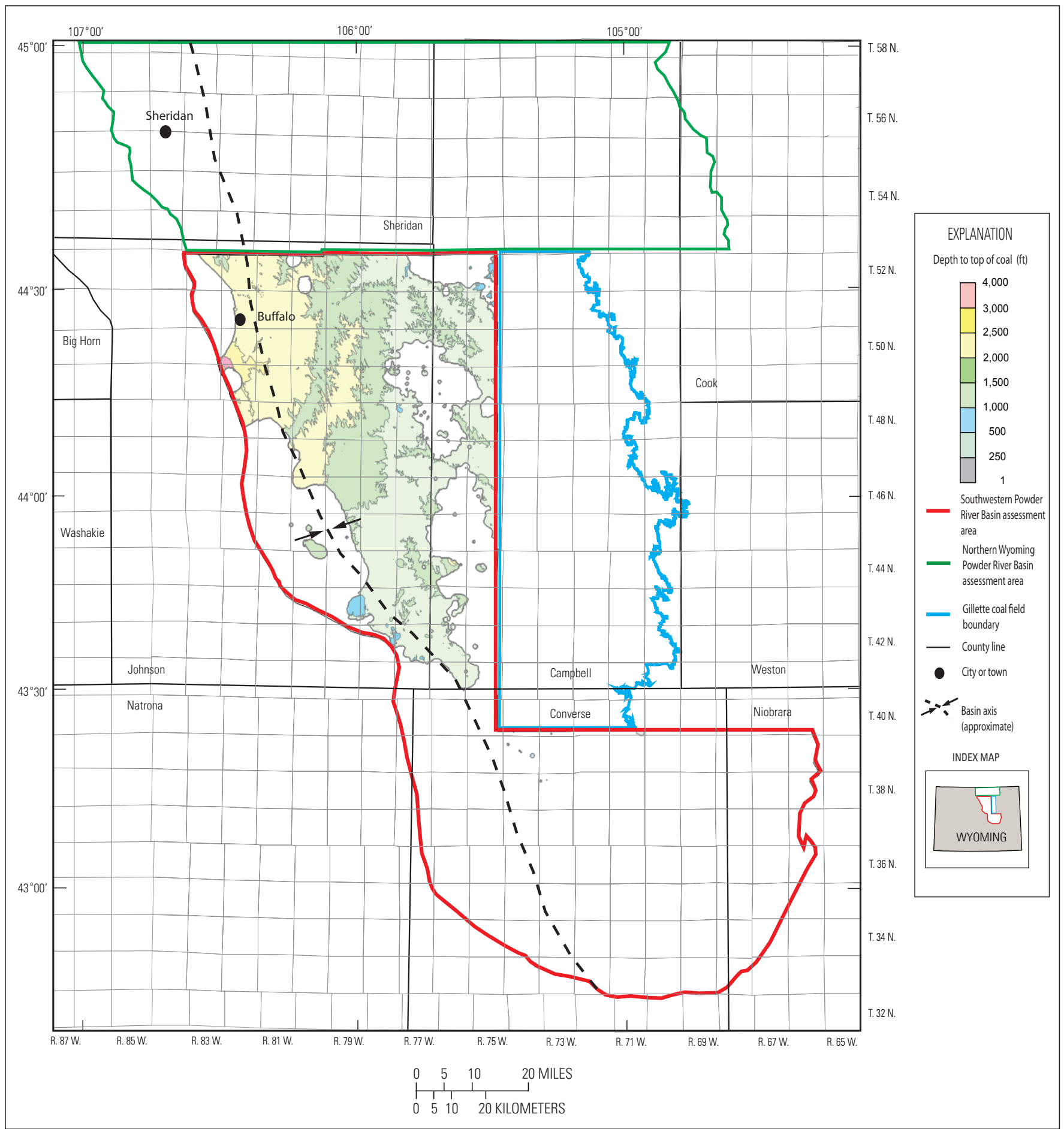

Figure 61. Depth to the top of the Canyon coal bed within the Southwestern Powder River Basin assessment area. 


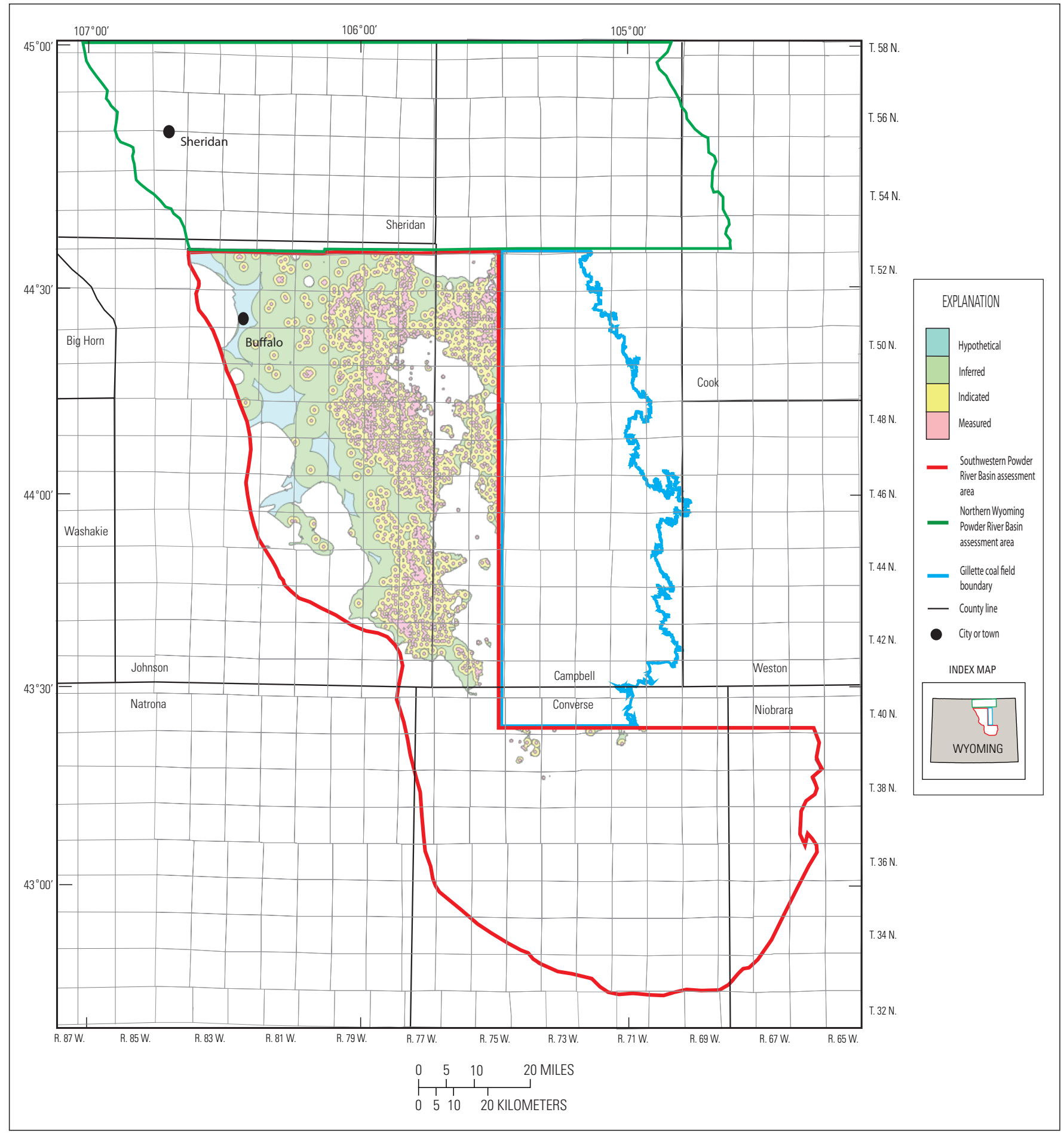

Figure 62. Coal resource reliability categories for the Canyon coal bed within the Southwestern Powder River Basin assessment area. 


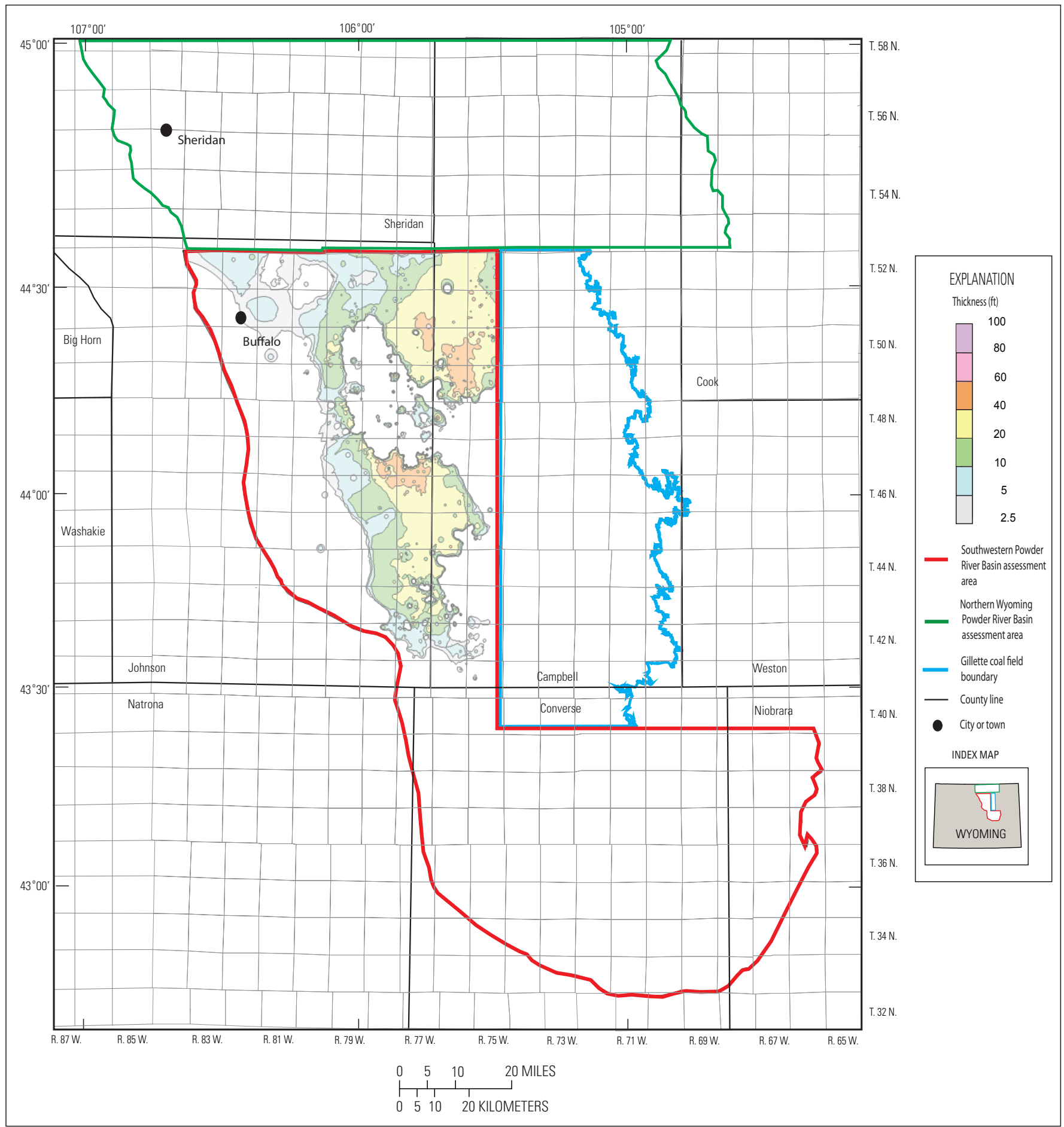

Figure 63. Isopach map of the Lower Canyon coal bed showing extent of resources greater than $2.5 \mathrm{ft}$ thick within the Southwestern Powder River Basin assessment area. 


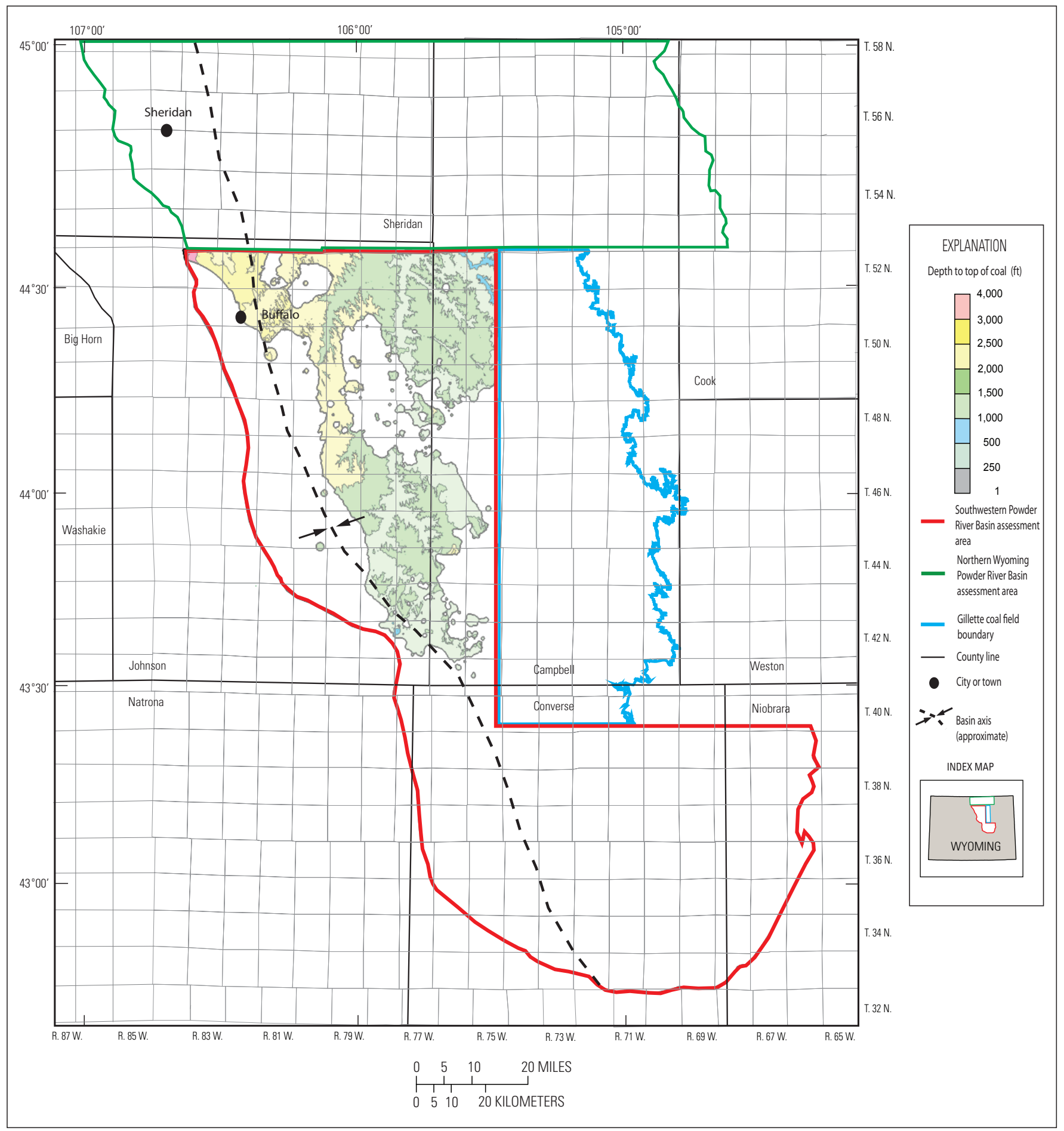

Figure 64. Depth to the top of the Lower Canyon coal bed within the Southwestern Powder River Basin assessment area. 


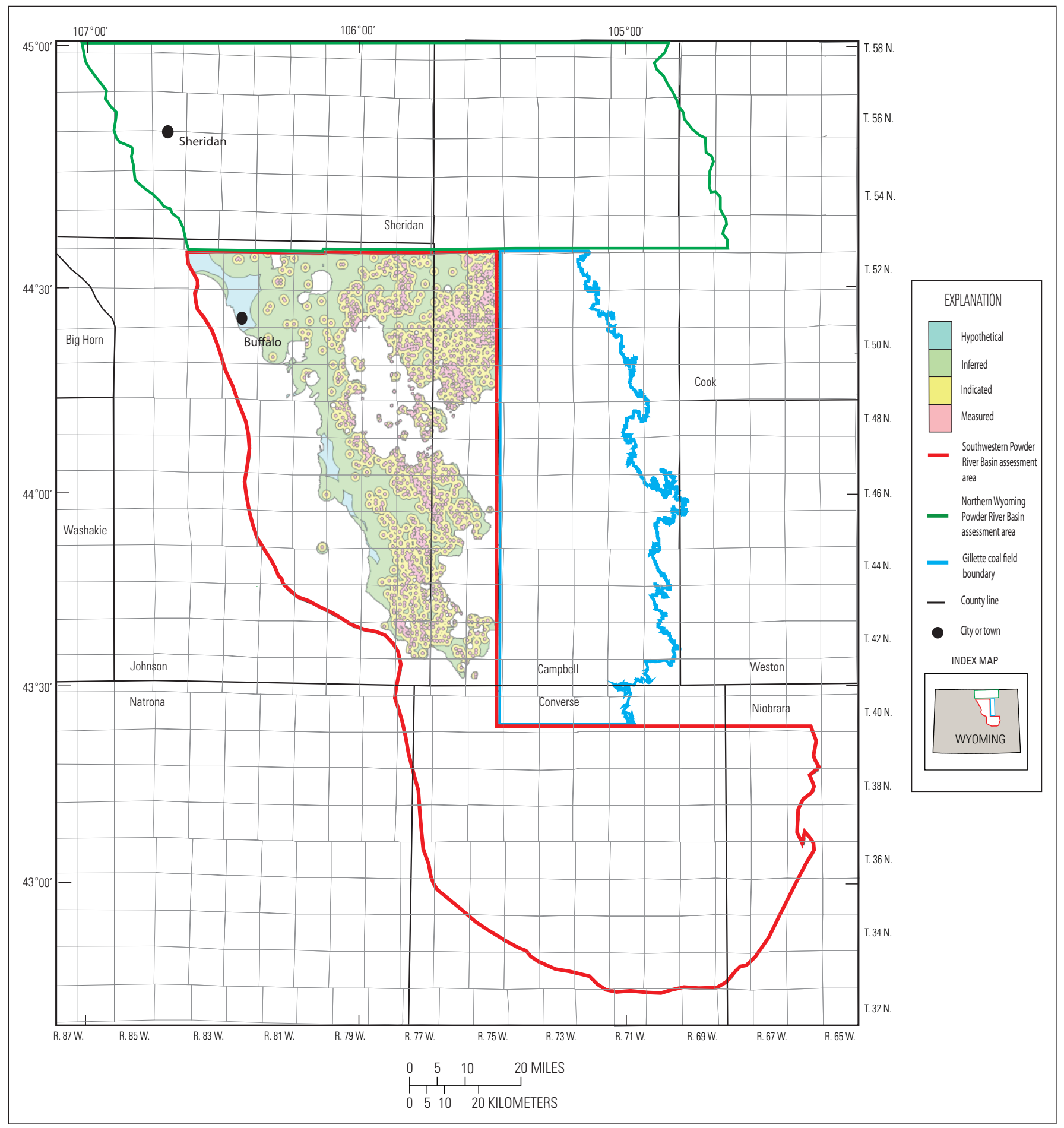

Figure 65. Coal resource reliability categories for the Lower Canyon coal bed within the Southwestern Powder River Basin assessment area. 


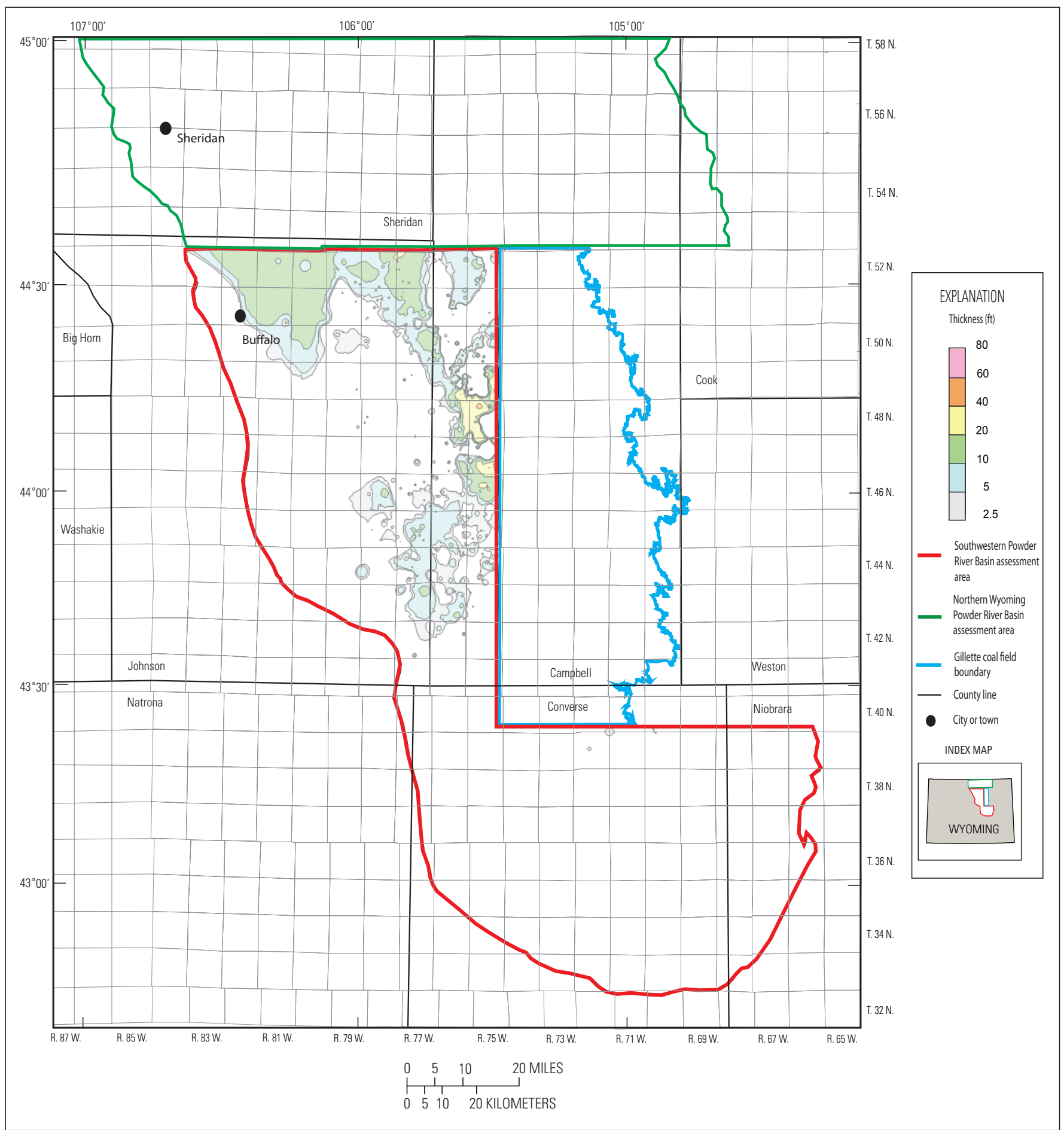

Figure 66. Isopach map of the Werner coal bed showing extent of resources greater than $2.5 \mathrm{ft}$ thick within the Southwestern Powder River Basin assessment area. 


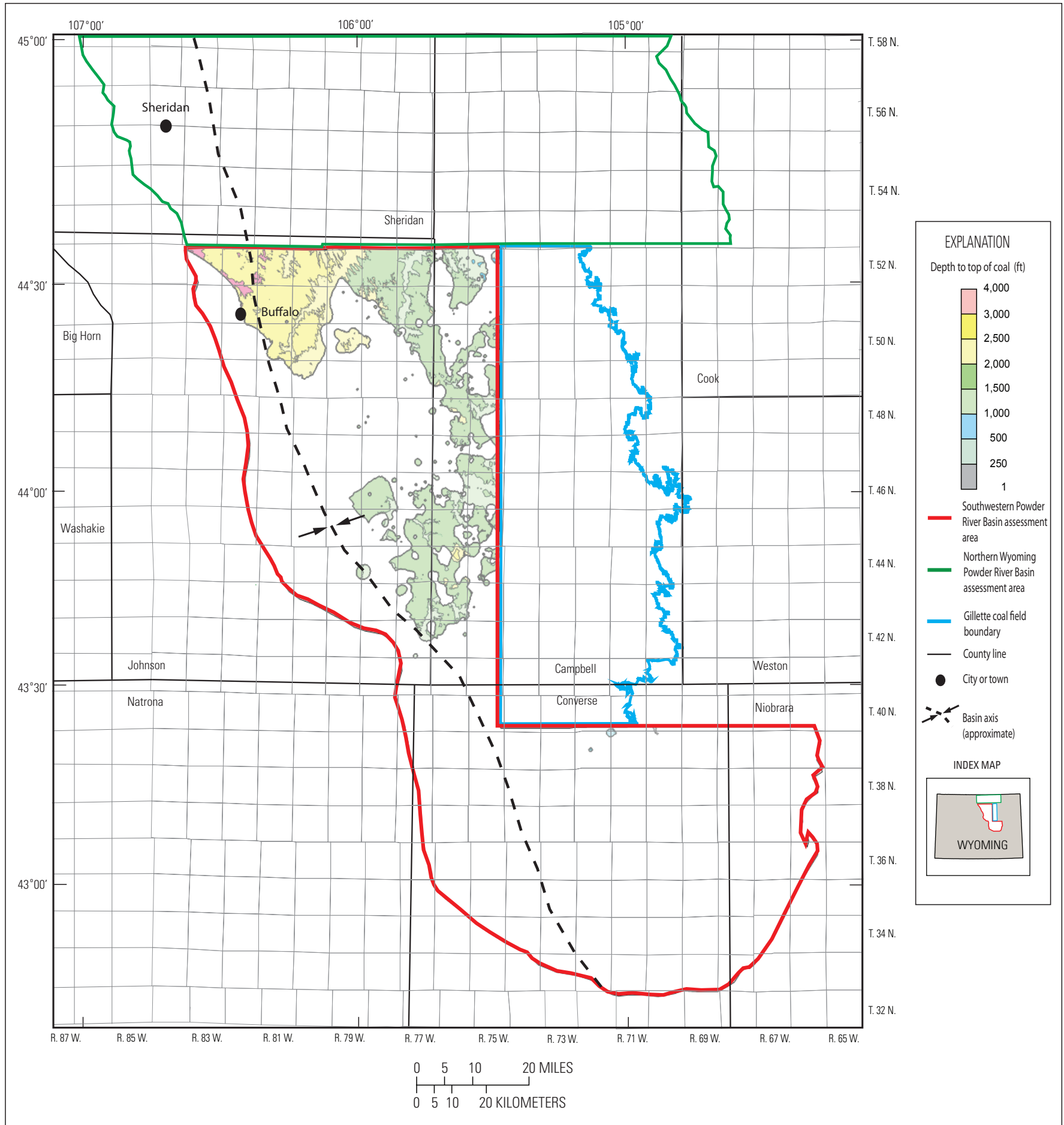

Figure 67. Depth to the top of the Werner coal bed within the Southwestern Powder River Basin assessment area. 


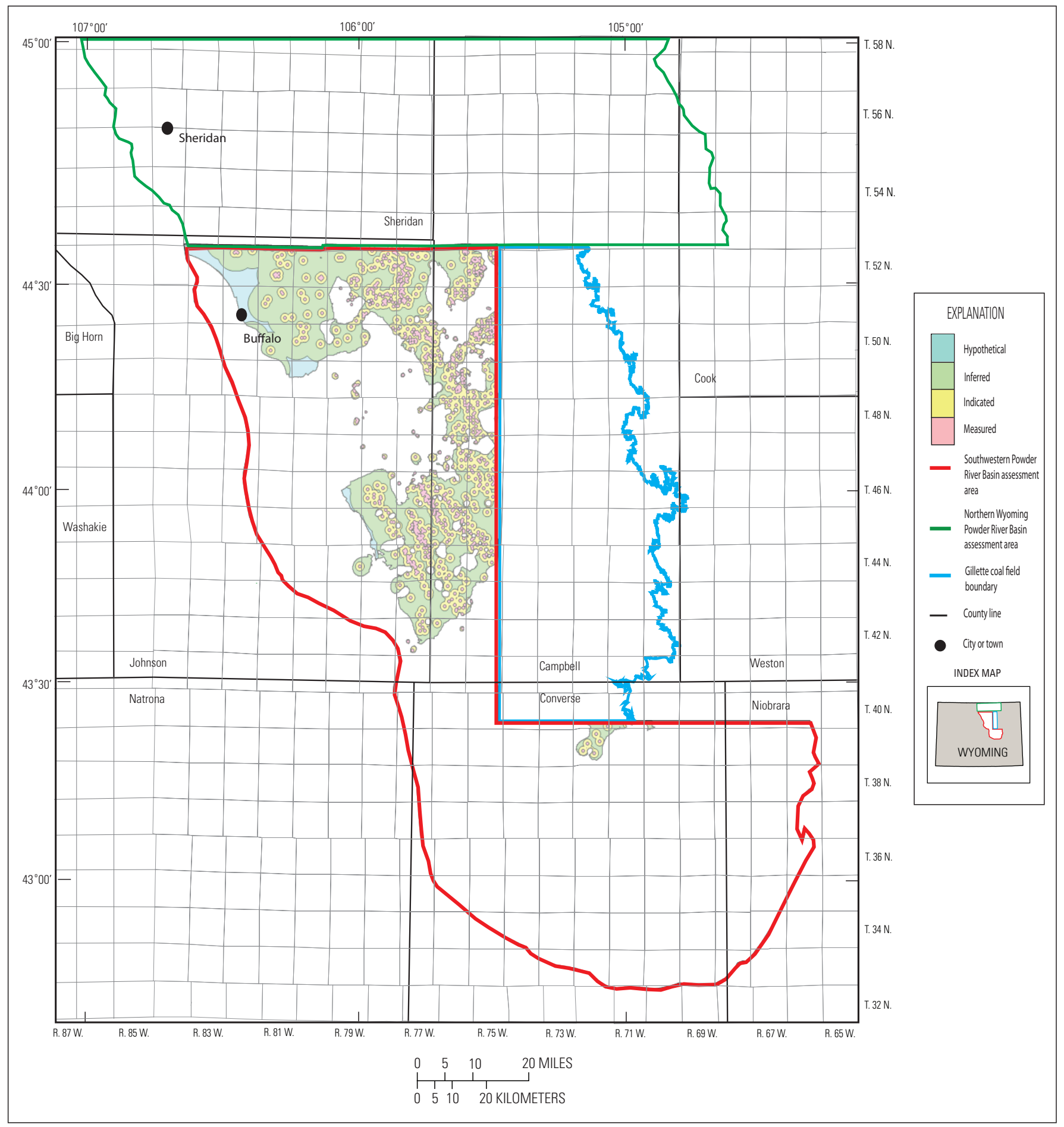

Figure 68. Coal resource reliability categories for the Werner coal bed within the Southwestern Powder River Basin assessment area. 


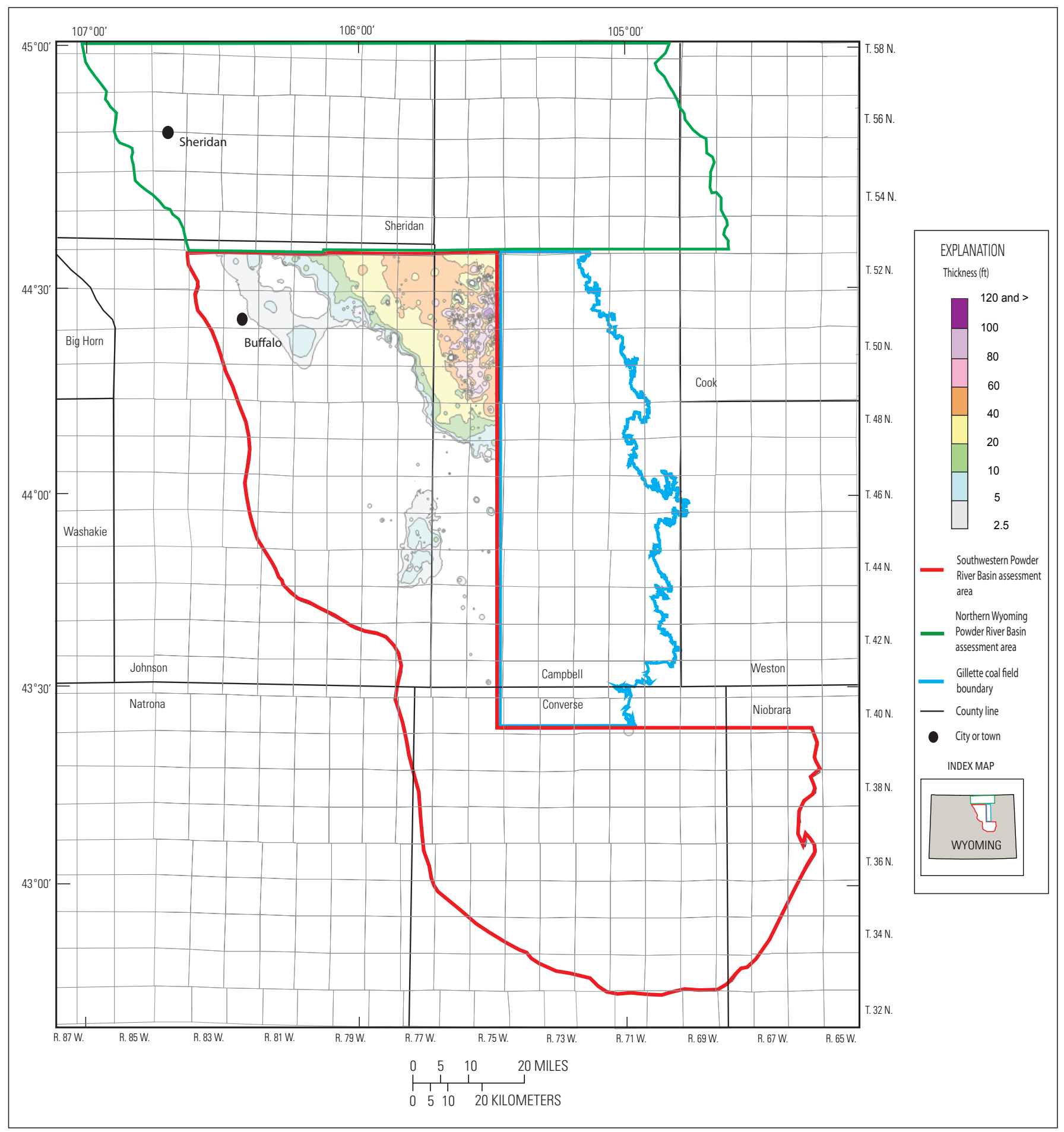

Figure 69. Isopach map of the Otter coal bed showing extent of resources greater than $2.5 \mathrm{ft}$ thick within the Southwestern Powder River Basin assessment area. 


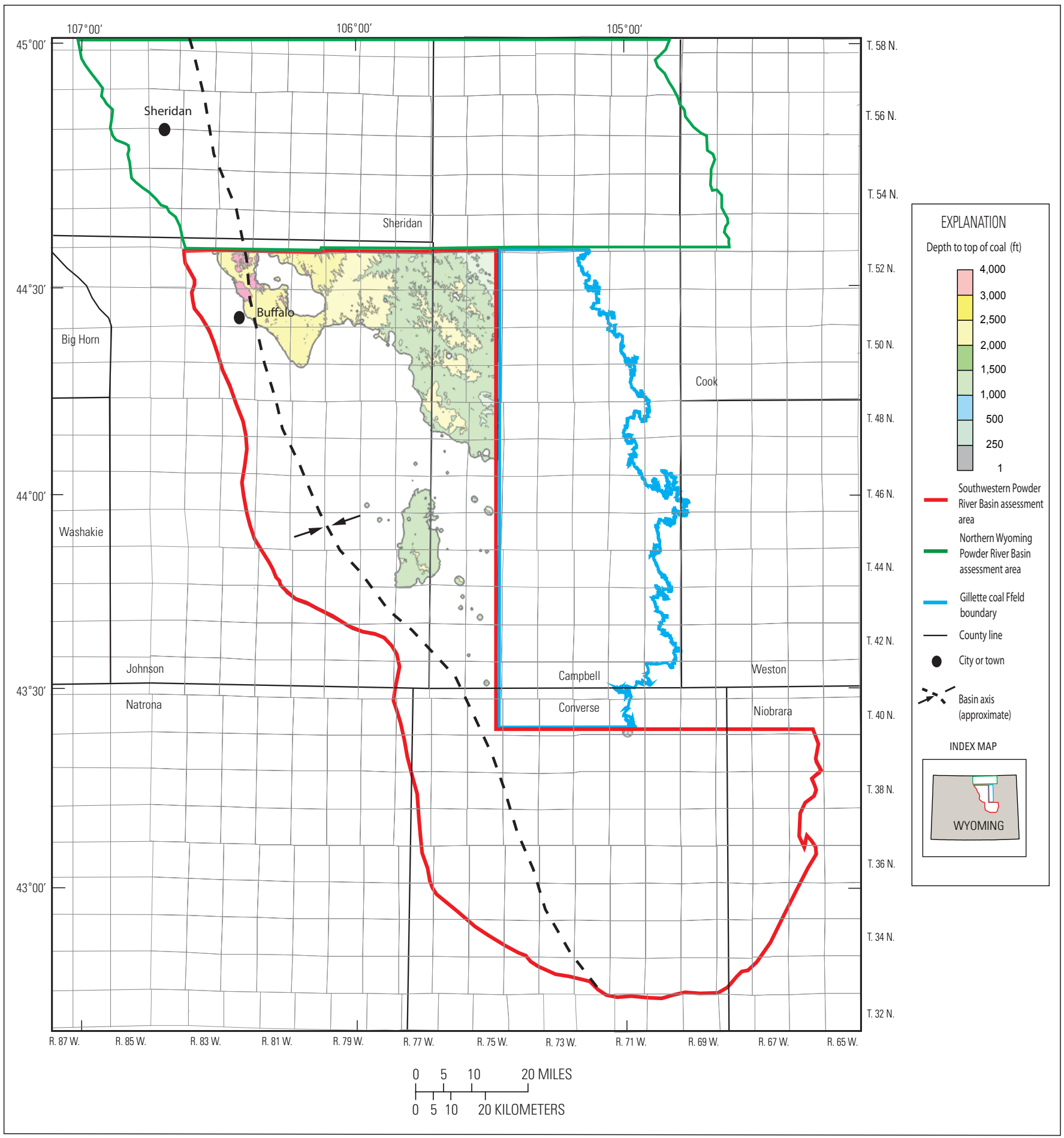

Figure 70. Depth to the top of the Otter coal bed within the Southwestern Powder River Basin assessment area. 


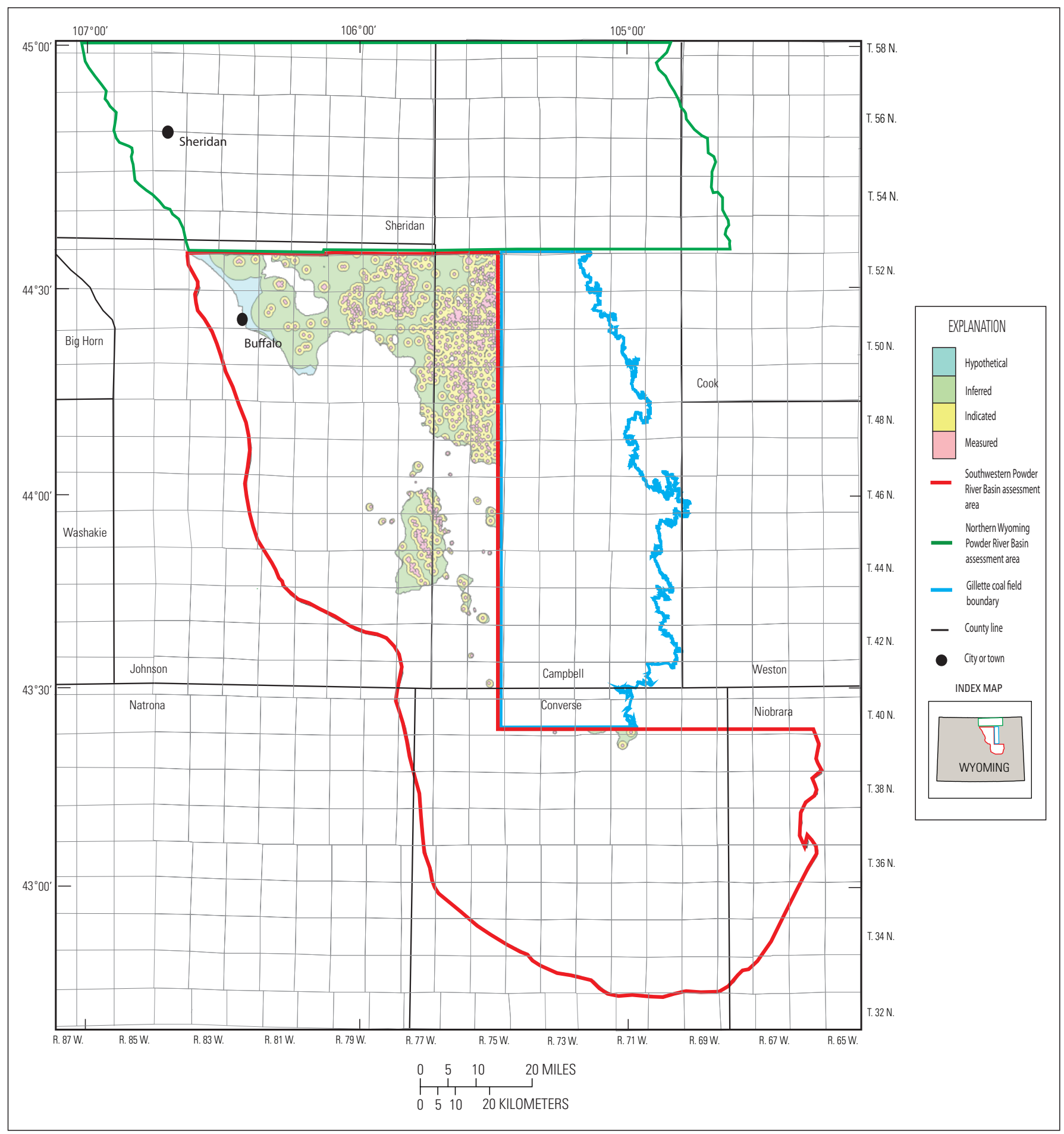

Figure 71. Coal resource reliability categories for the Otter coal bed within the Southwestern Powder River Basin assessment area. 


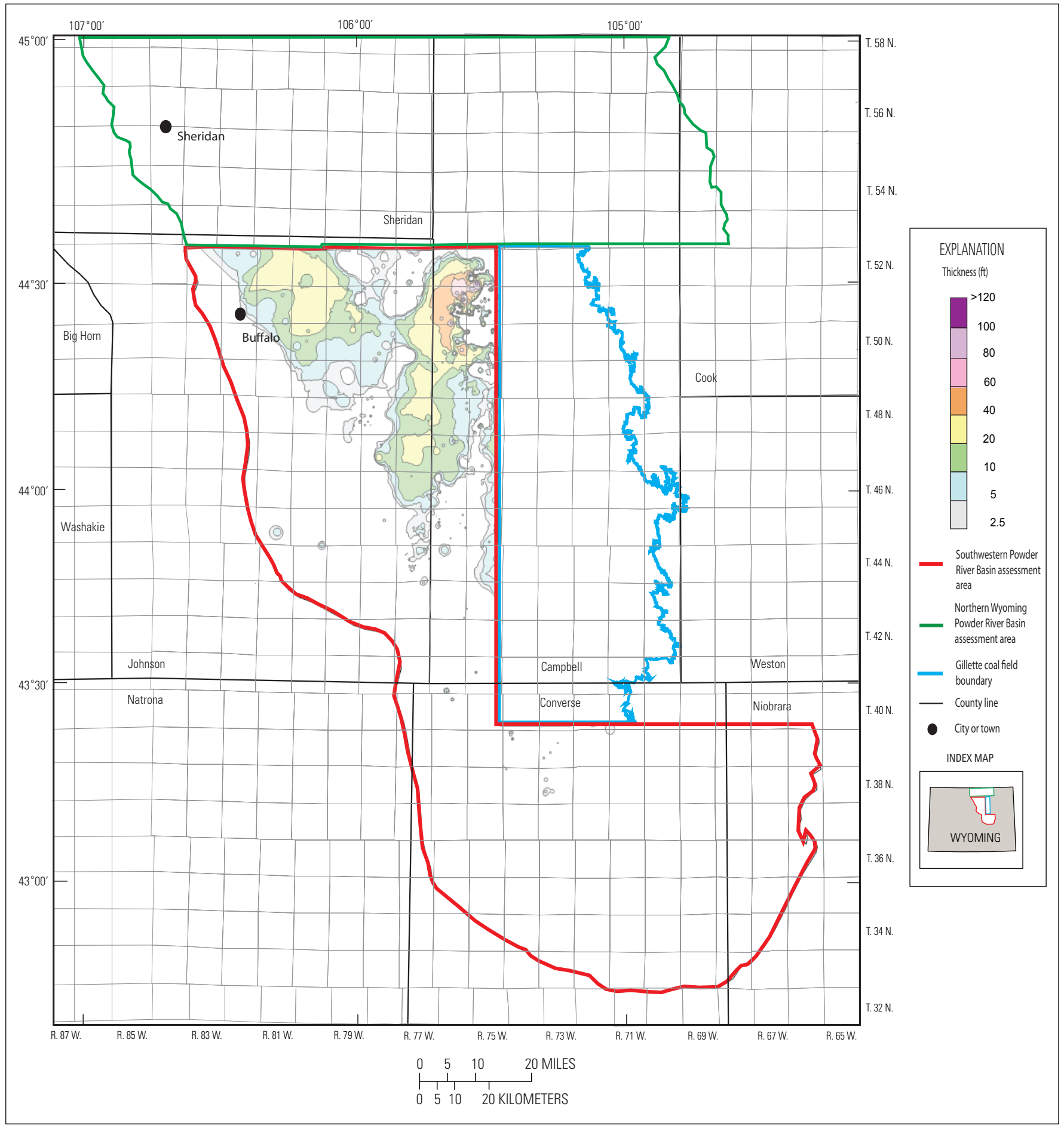

Figure 72. Isopach map of the Gates coal bed showing extent of resources greater than $2.5 \mathrm{ft}$ thick within the Southwestern Powder River Basin assessment area. 


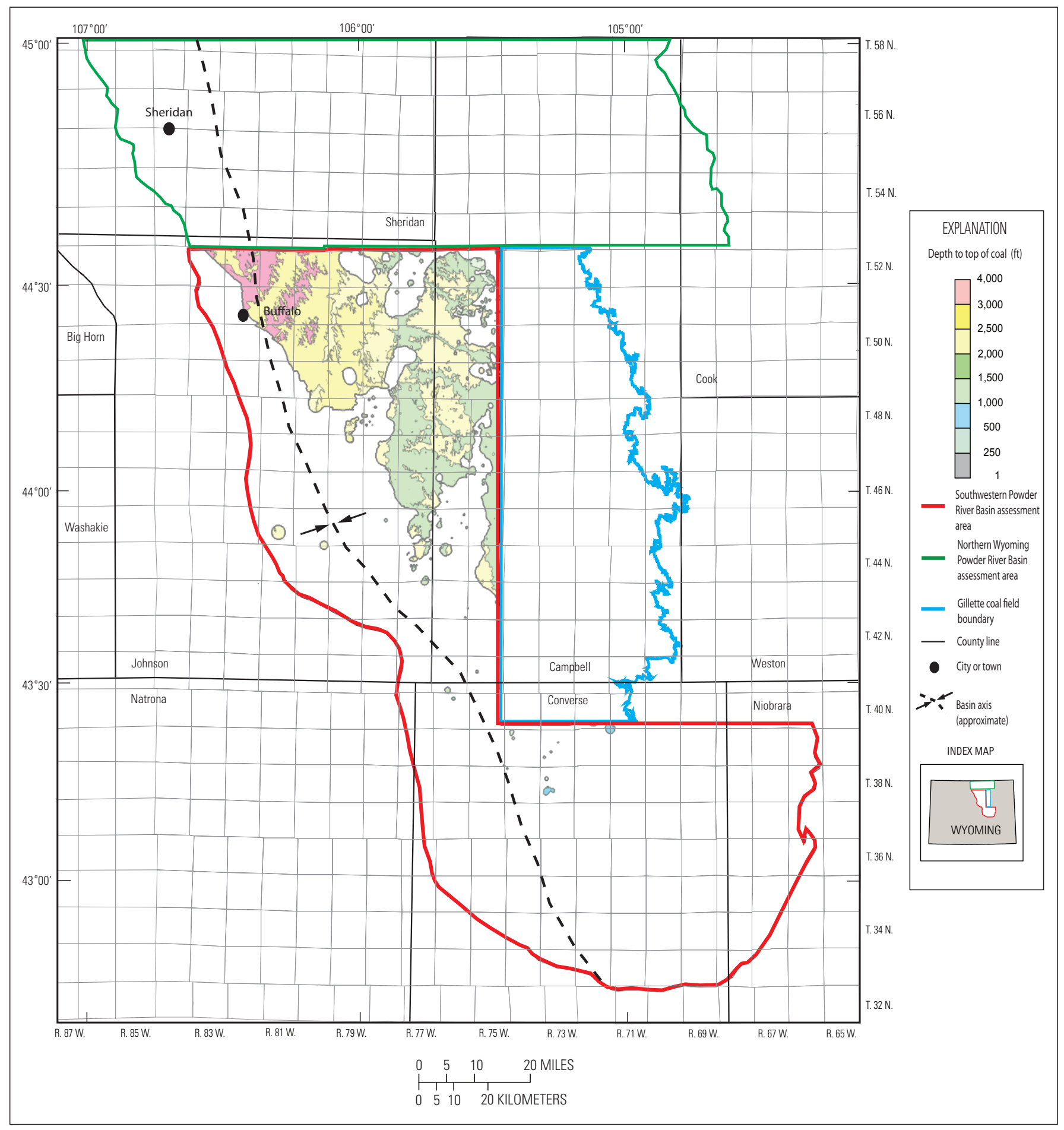

Figure 73. Depth to the top of the Gates coal bed within the Southwestern Powder River Basin assessment area. 


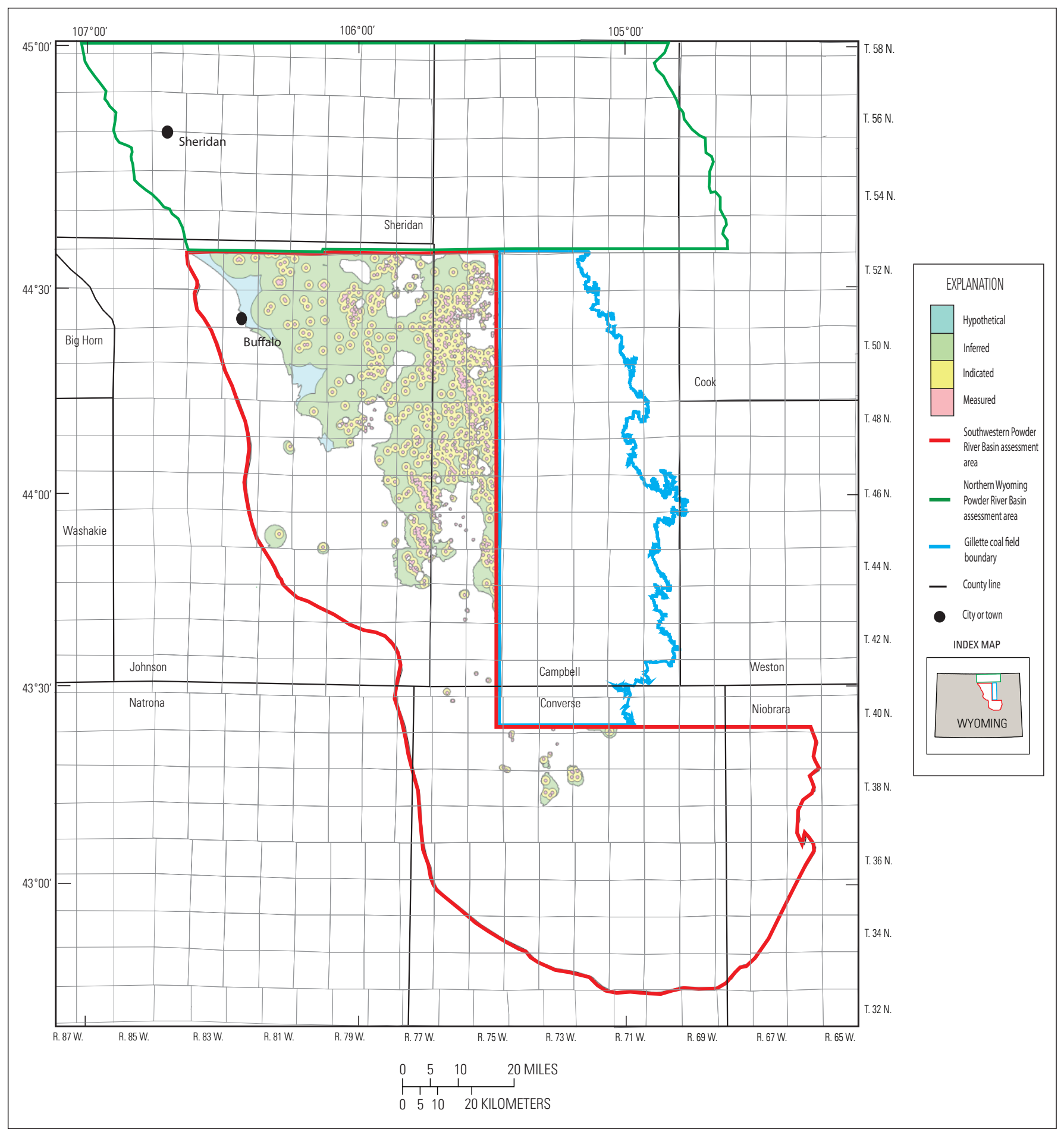

Figure 74. Coal resource reliability categories for the Gates coal bed within the Southwestern Powder River Basin assessment area. 


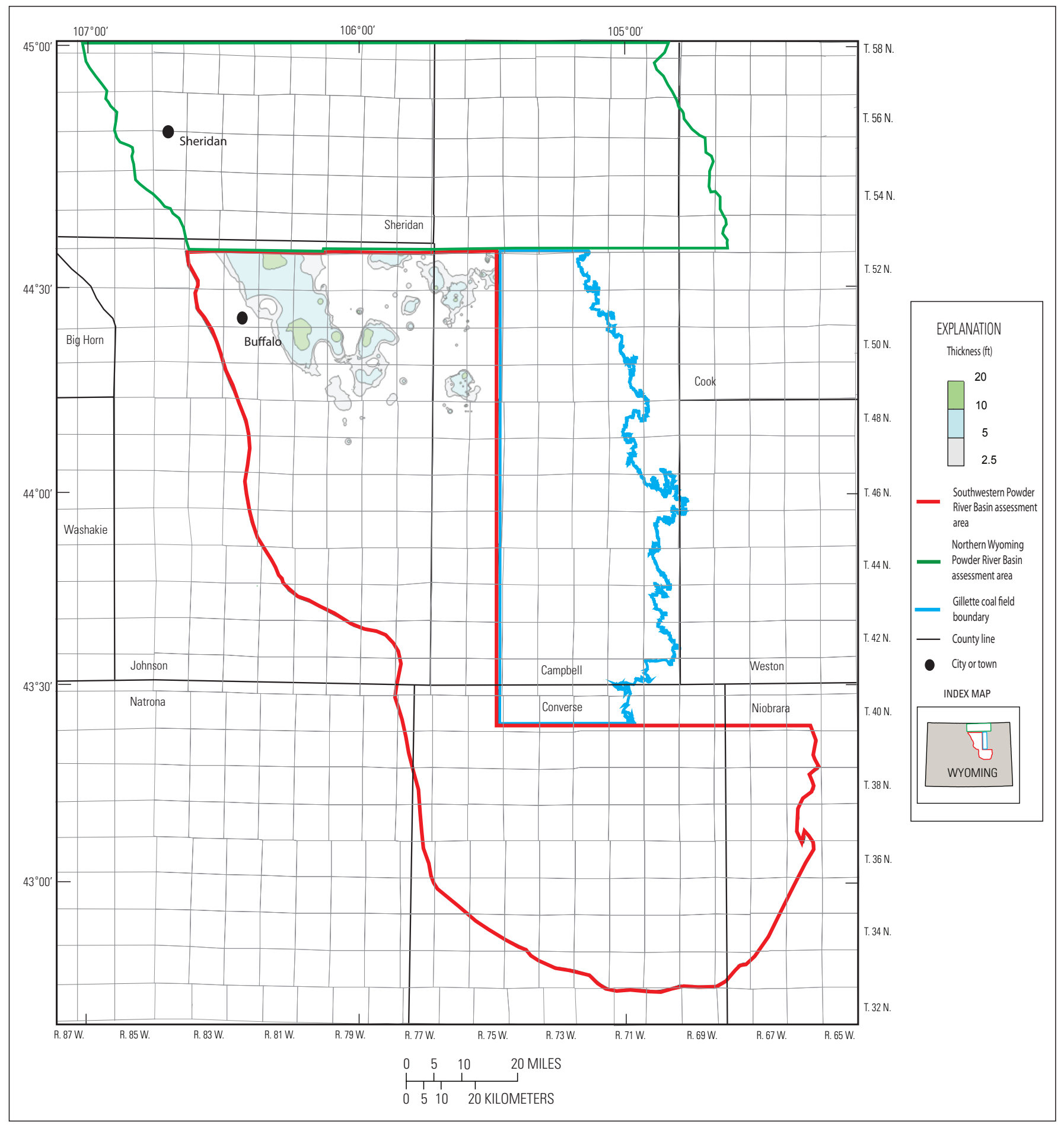

Figure 75. Isopach map of the Pawnee coal bed showing extent of resources greater than $2.5 \mathrm{ft}$ thick within the Southwestern Powder River Basin assessment area. 


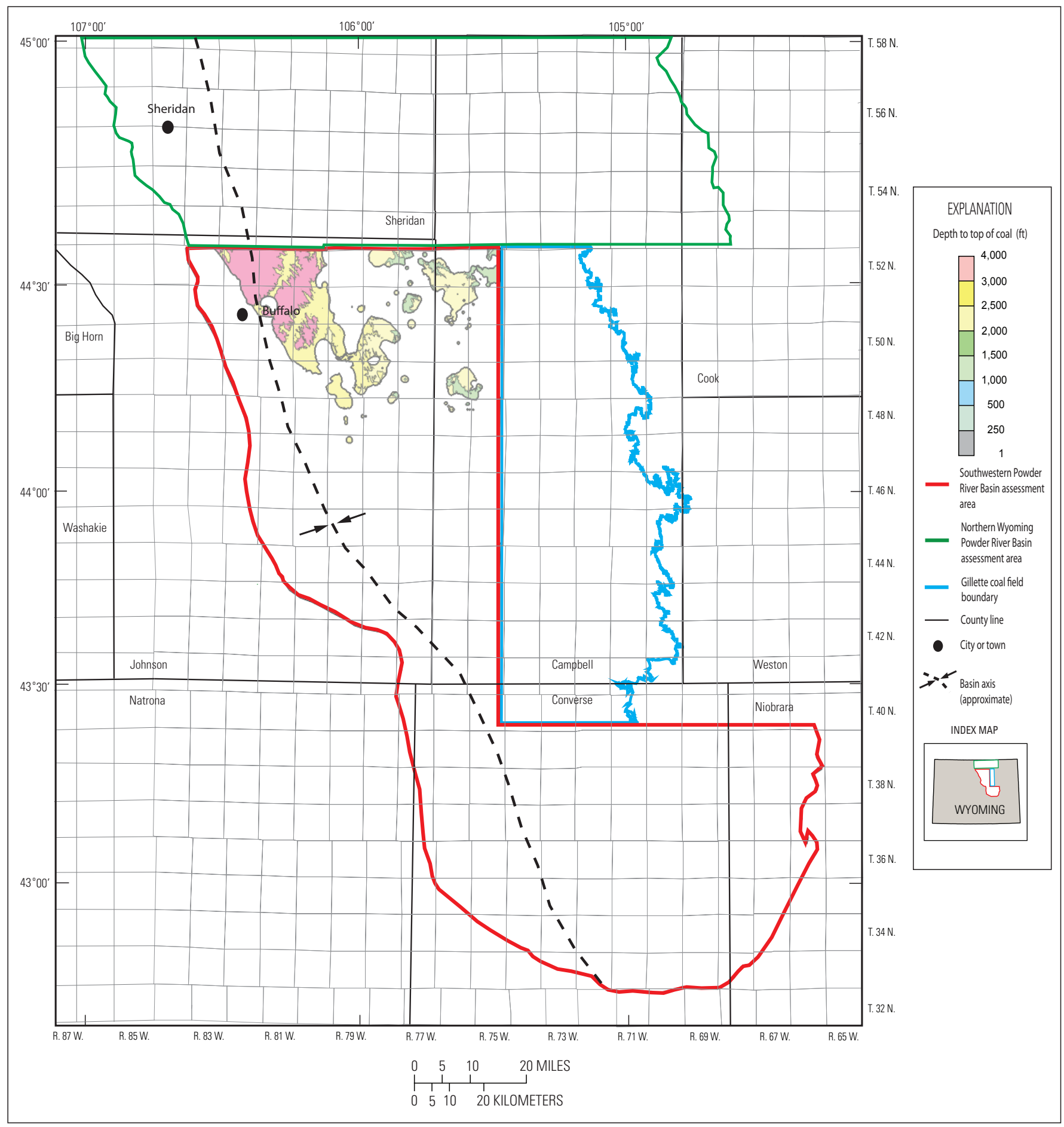

Figure 76. Depth to the top of the Pawnee coal bed within the Southwestern Powder River Basin assessment area. 


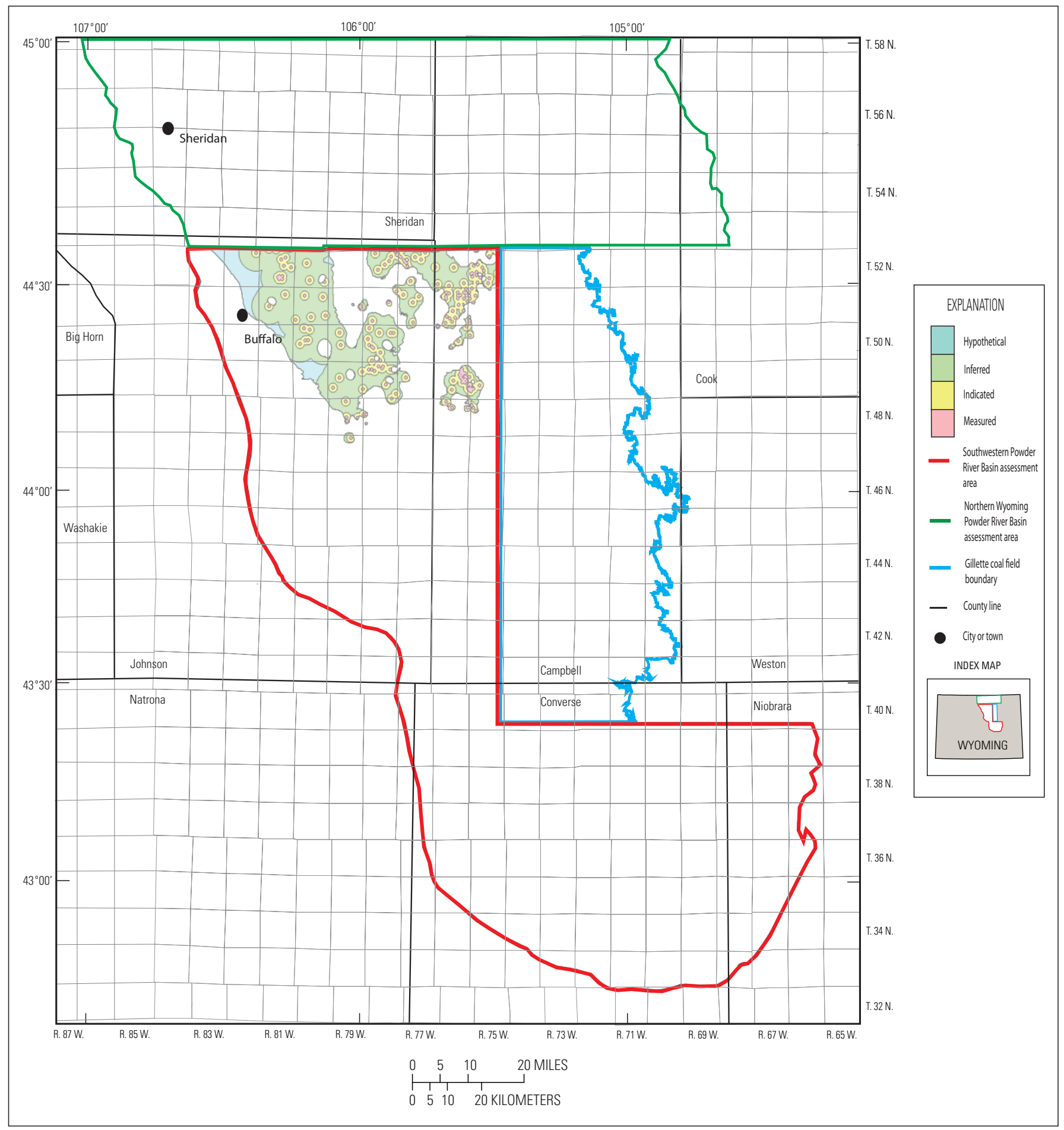

Figure 77. Coal resource reliability categories for the Pawnee coal bed within the Southwestern Powder River Basin assessment area. 


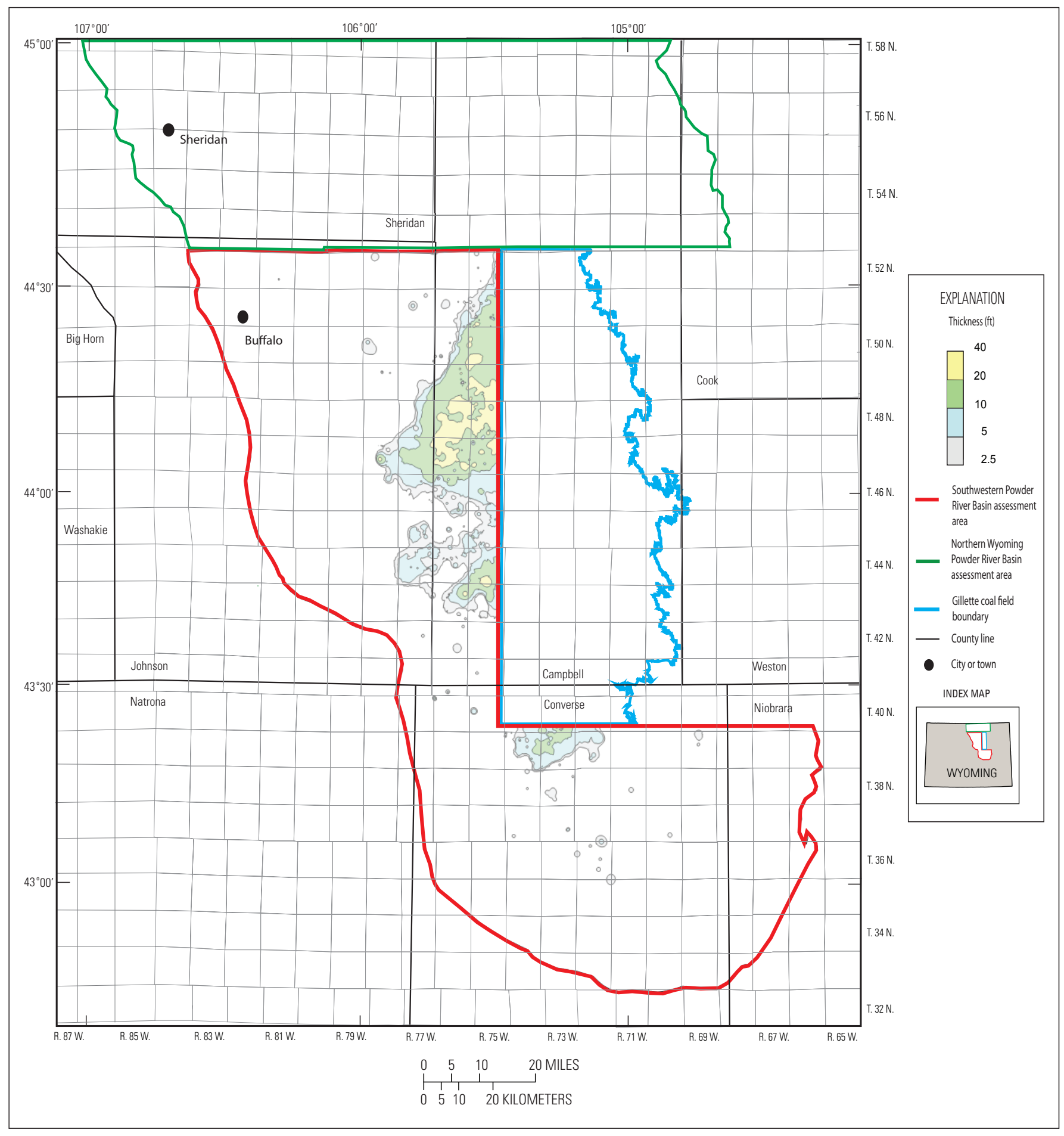

Figure 78. Isopach map of the Deep 1 coal bed showing extent of resources greater than $2.5 \mathrm{ft}$ thick within the Southwestern Powder River Basin assessment area. 


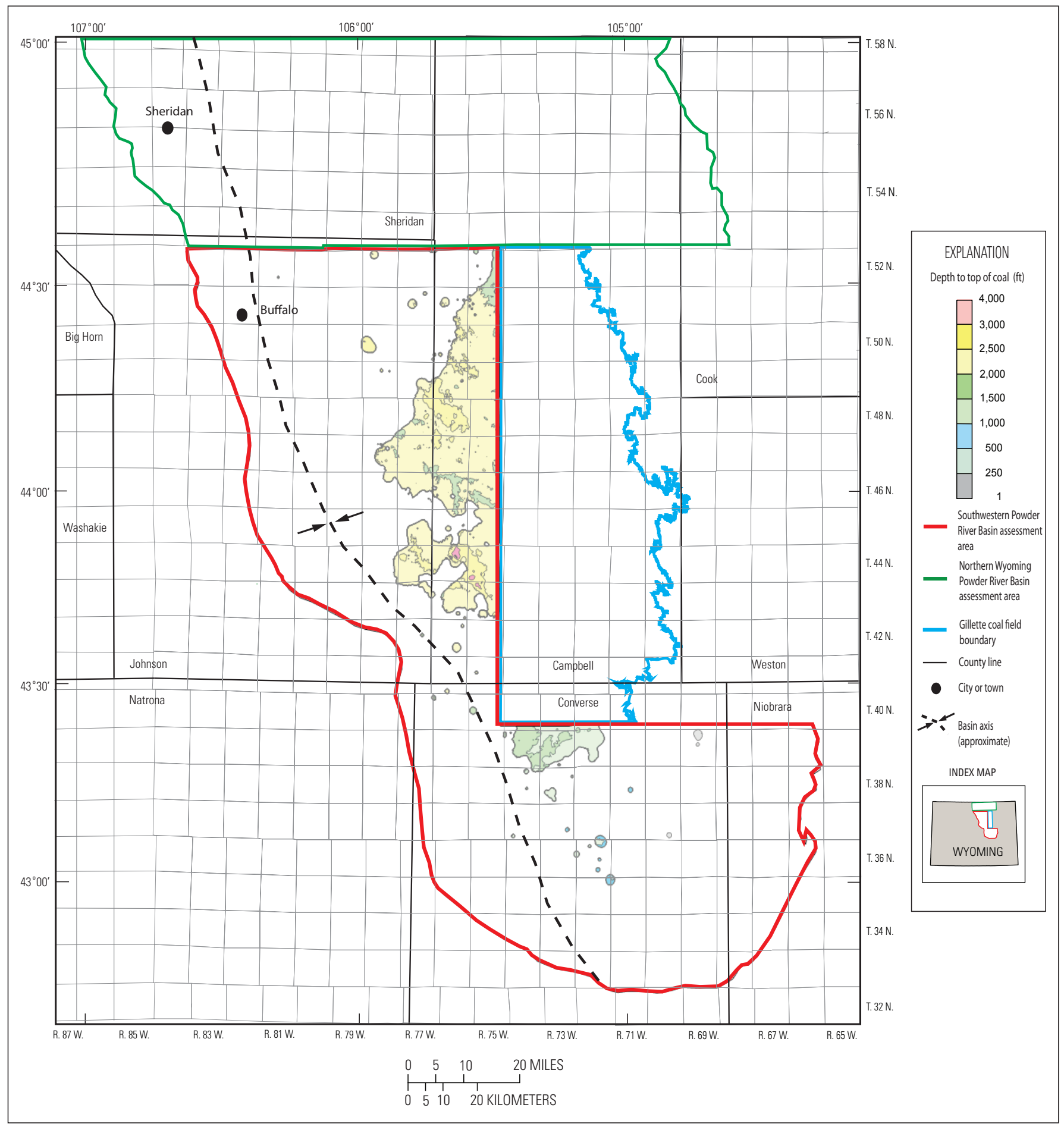

Figure 79. Depth to the top of the Deep 1 coal bed within the Southwestern Powder River Basin assessment area. 


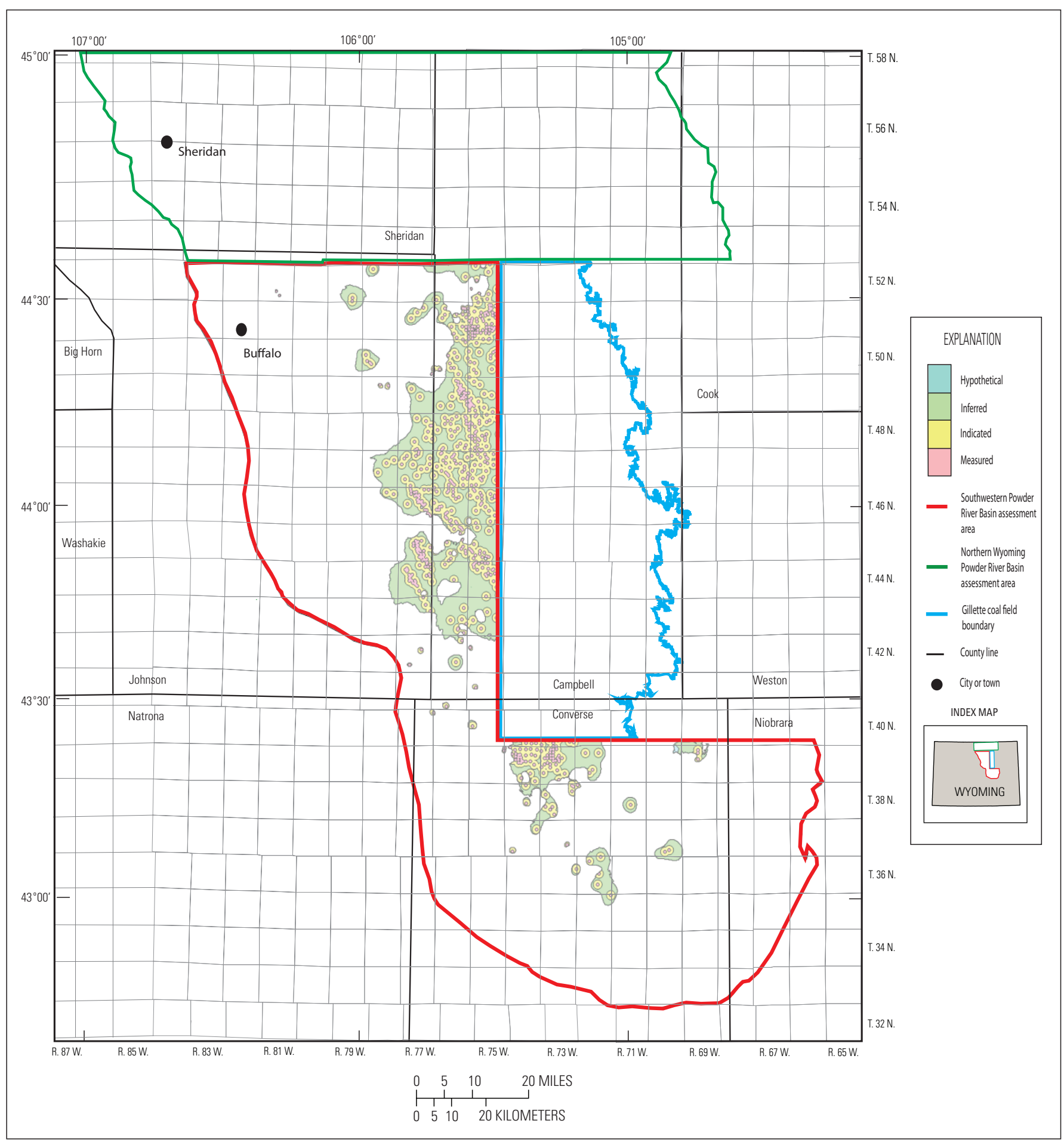

Figure 80. Coal resource reliability categories for the Deep 1 coal bed within the Southwestern Powder River Basin assessment area. 


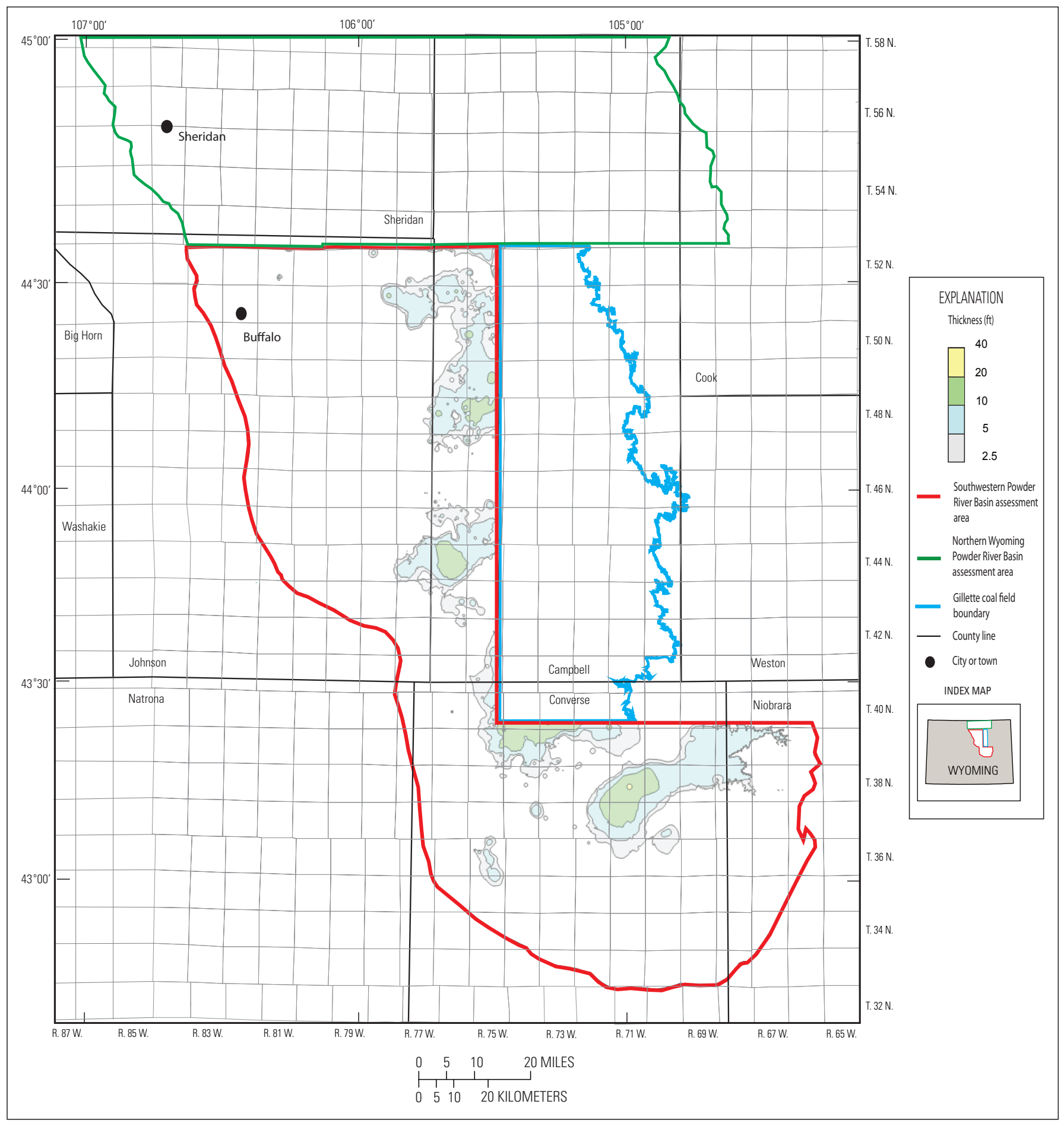

Figure 81. Isopach map of the Deep 2 coal bed showing extent of resources greater than $2.5 \mathrm{ft}$ thick within the Southwestern Powder River Basin assessment area. 


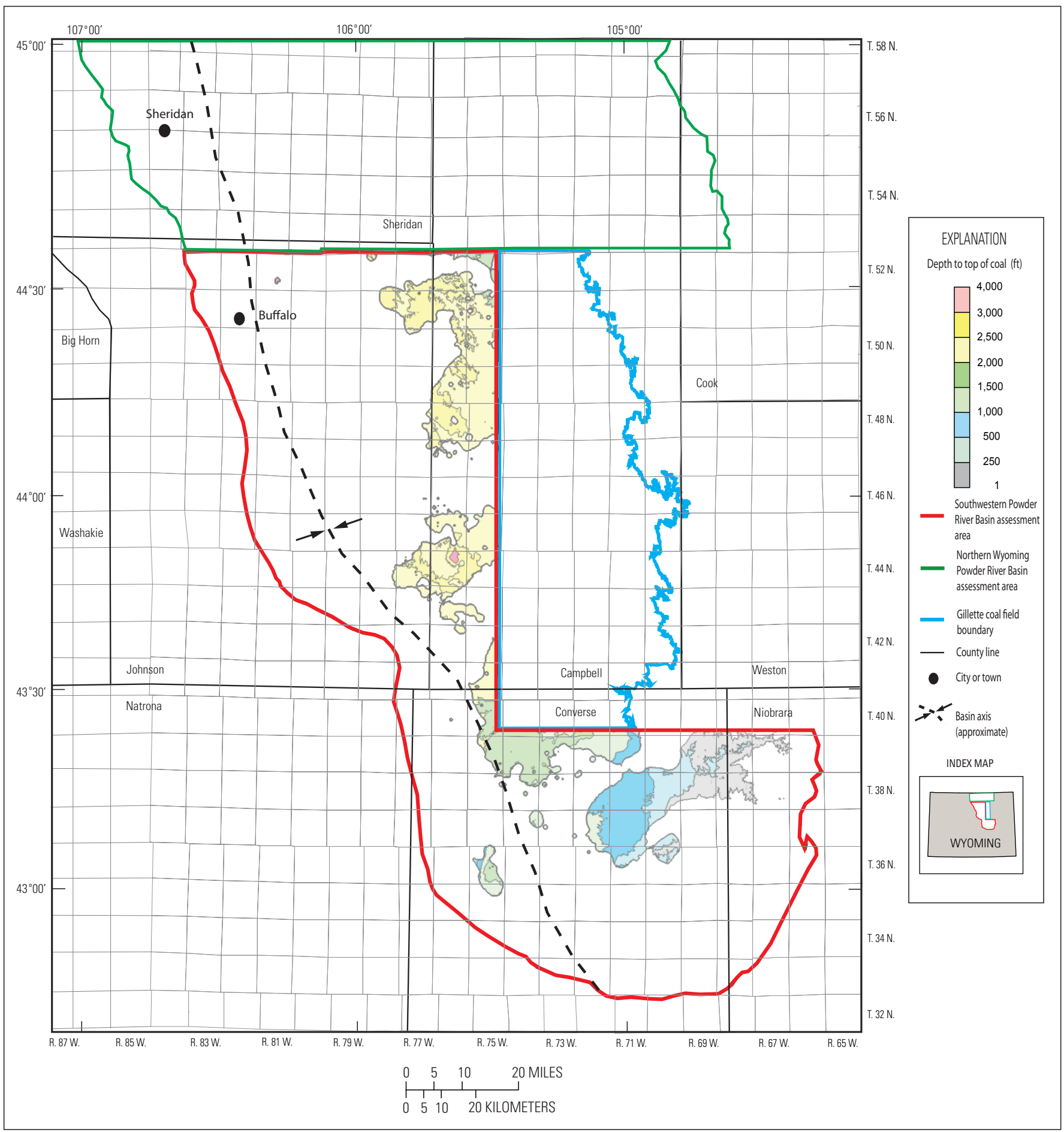

Figure 82. Depth to the top of the Deep 2 coal bed within the Southwestern Powder River Basin assessment area. 


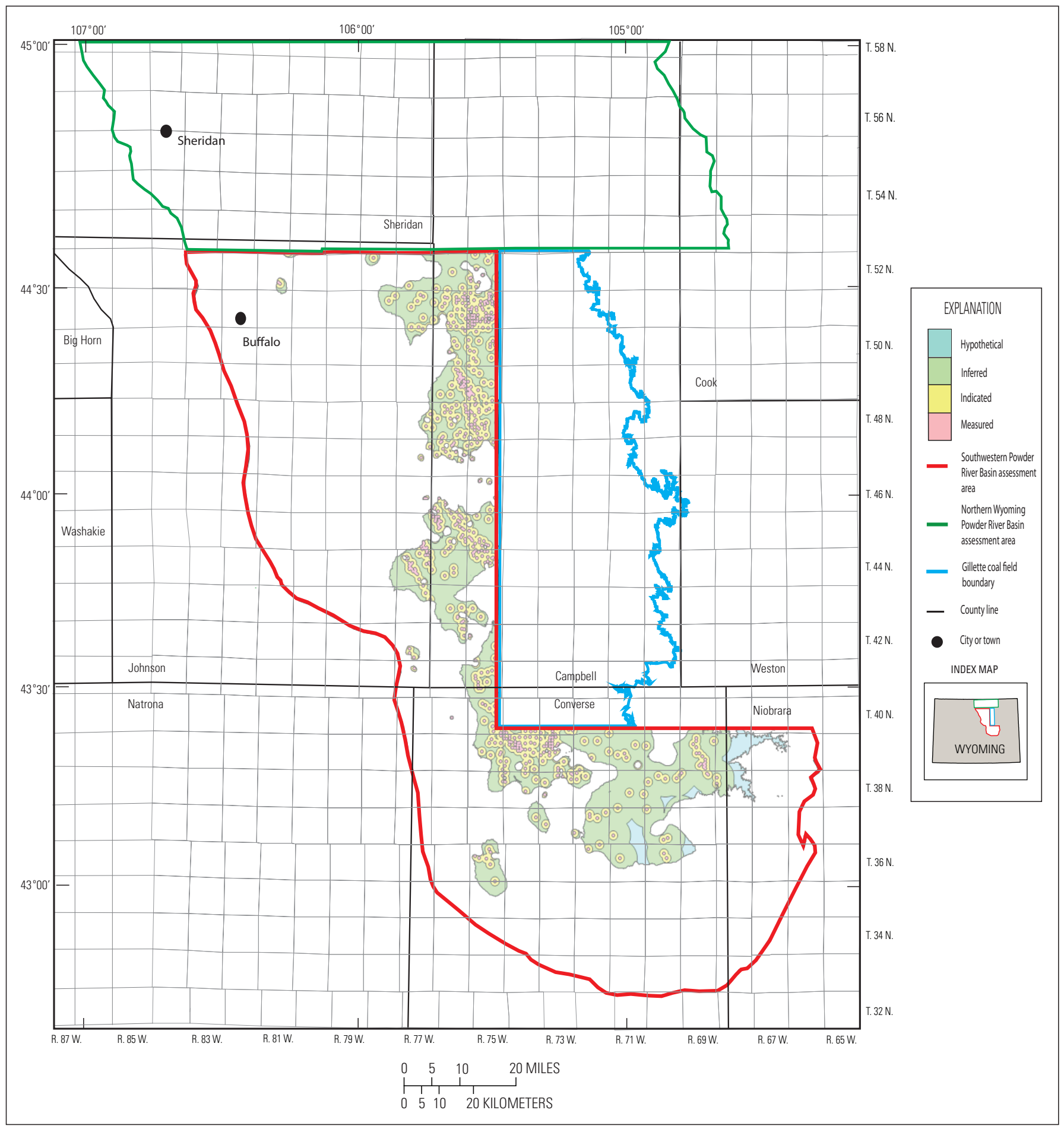

Figure 83. Coal resource reliability categories for the Deep 2 coal bed within the Southwestern Powder River Basin assessment area. 


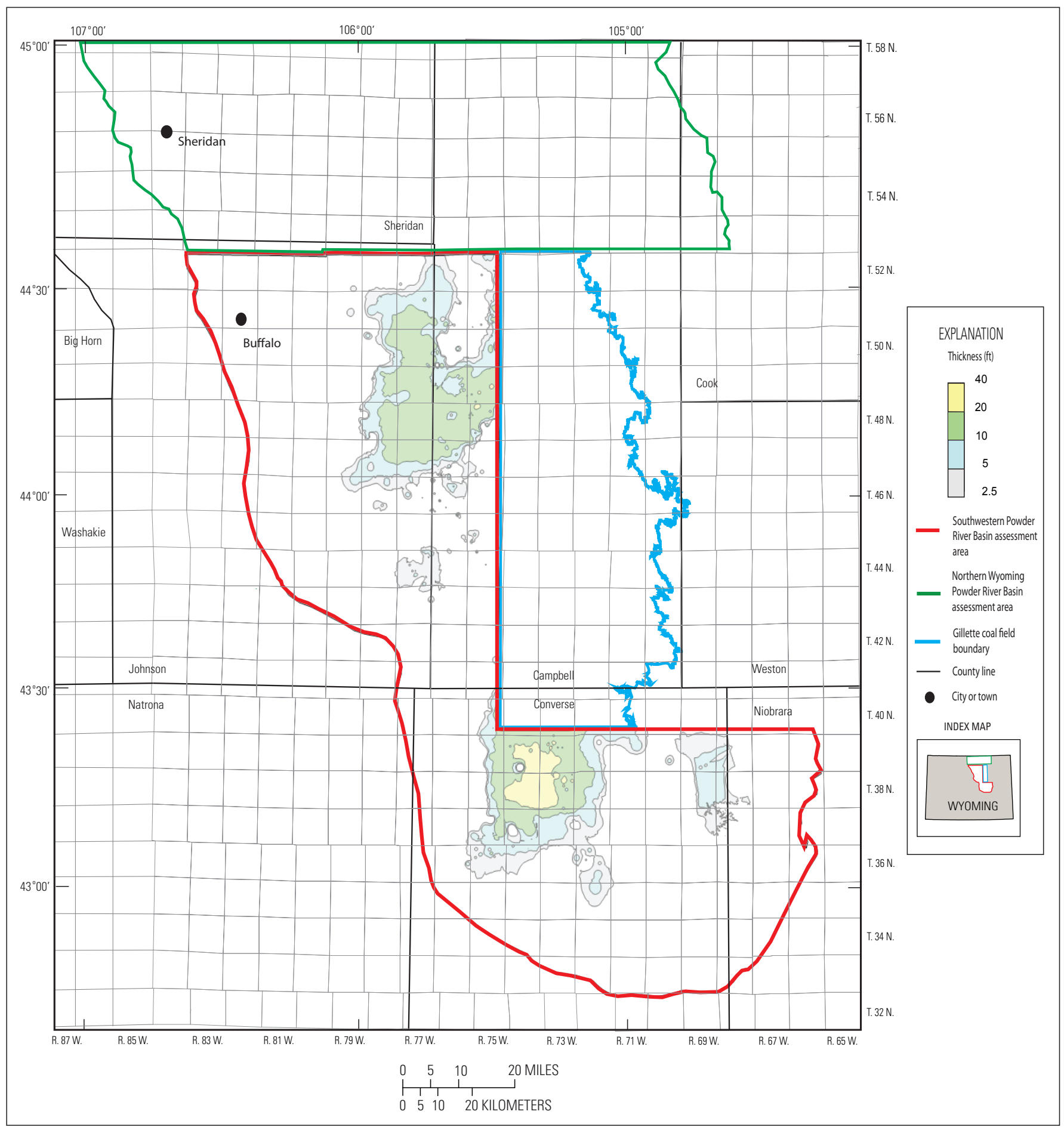

Figure 84. Isopach map of the Deep 3 coal bed showing extent of resources greater than $2.5 \mathrm{ft}$ thick within the Southwestern Powder River Basin assessment area. 


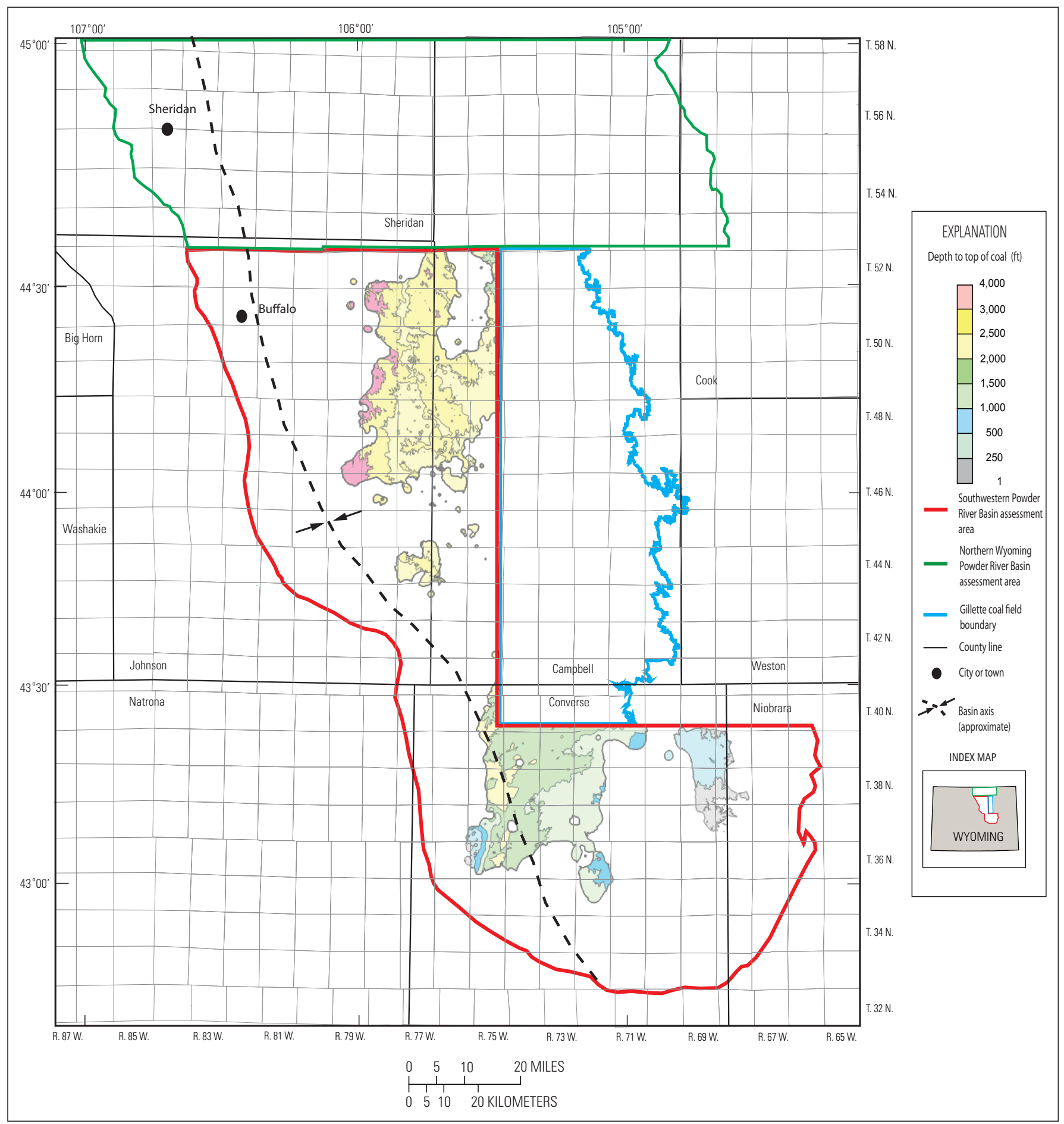

Figure 85. Depth to the top of the Deep 3 coal bed within the Southwestern Powder River Basin assessment area. 


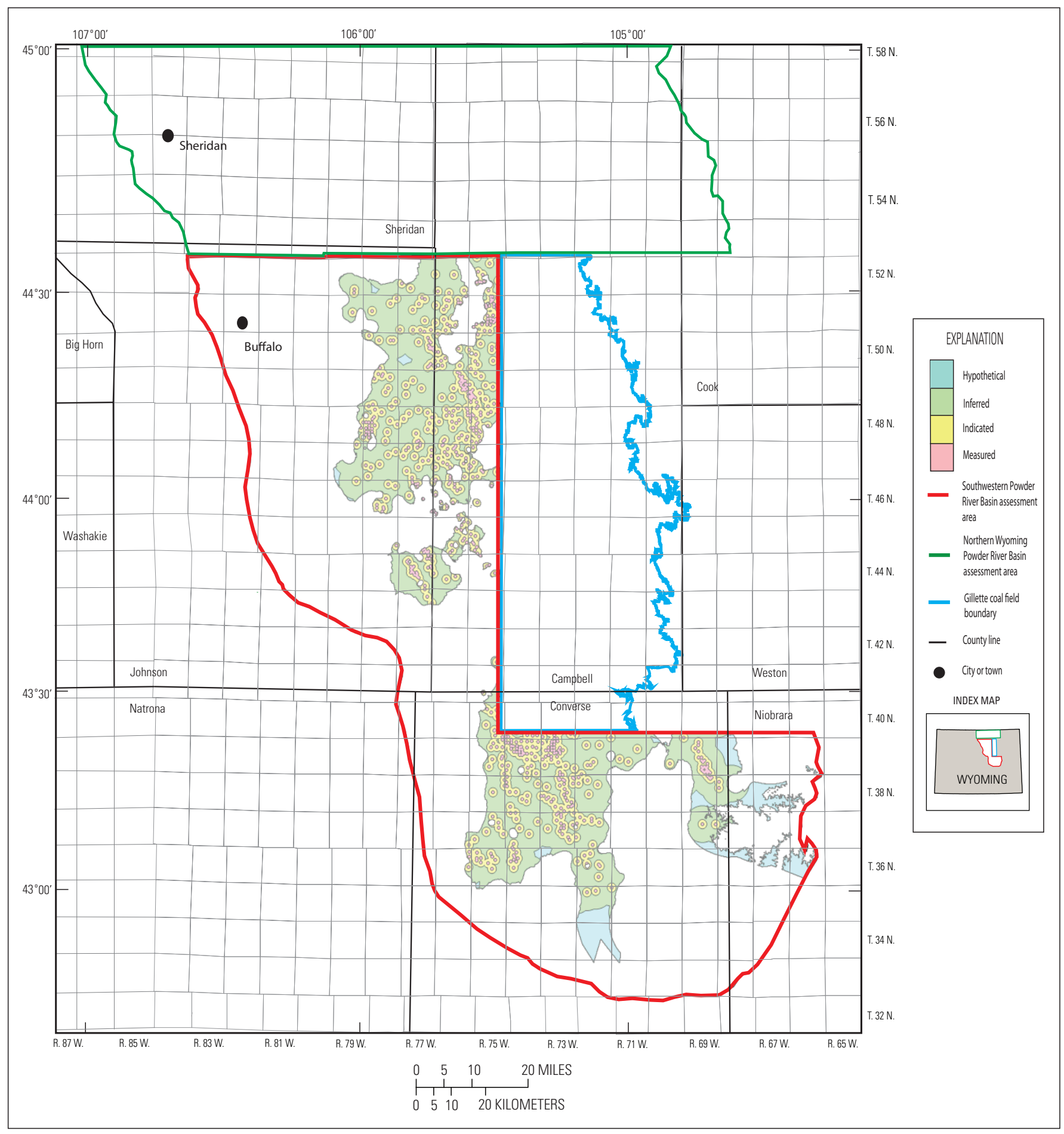

Figure 86. Coal resource reliability categories for the Deep 3 coal bed within the Southwestern Powder River Basin assessment area. 


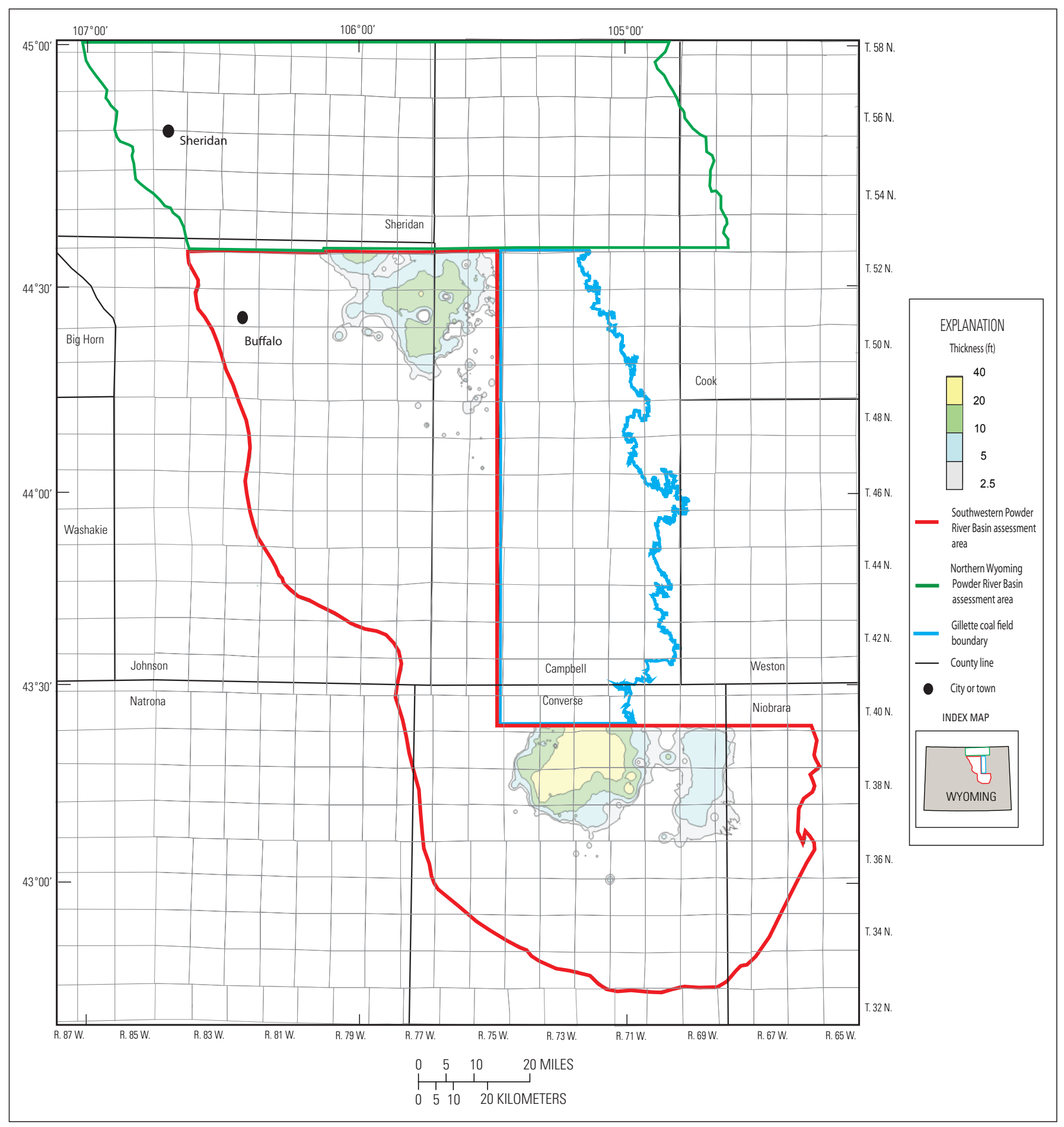

Figure 87. Isopach map of the Roberts coal bed showing extent of resources greater than $2.5 \mathrm{ft}$ thick within the Southwestern Powder River Basin assessment area. 


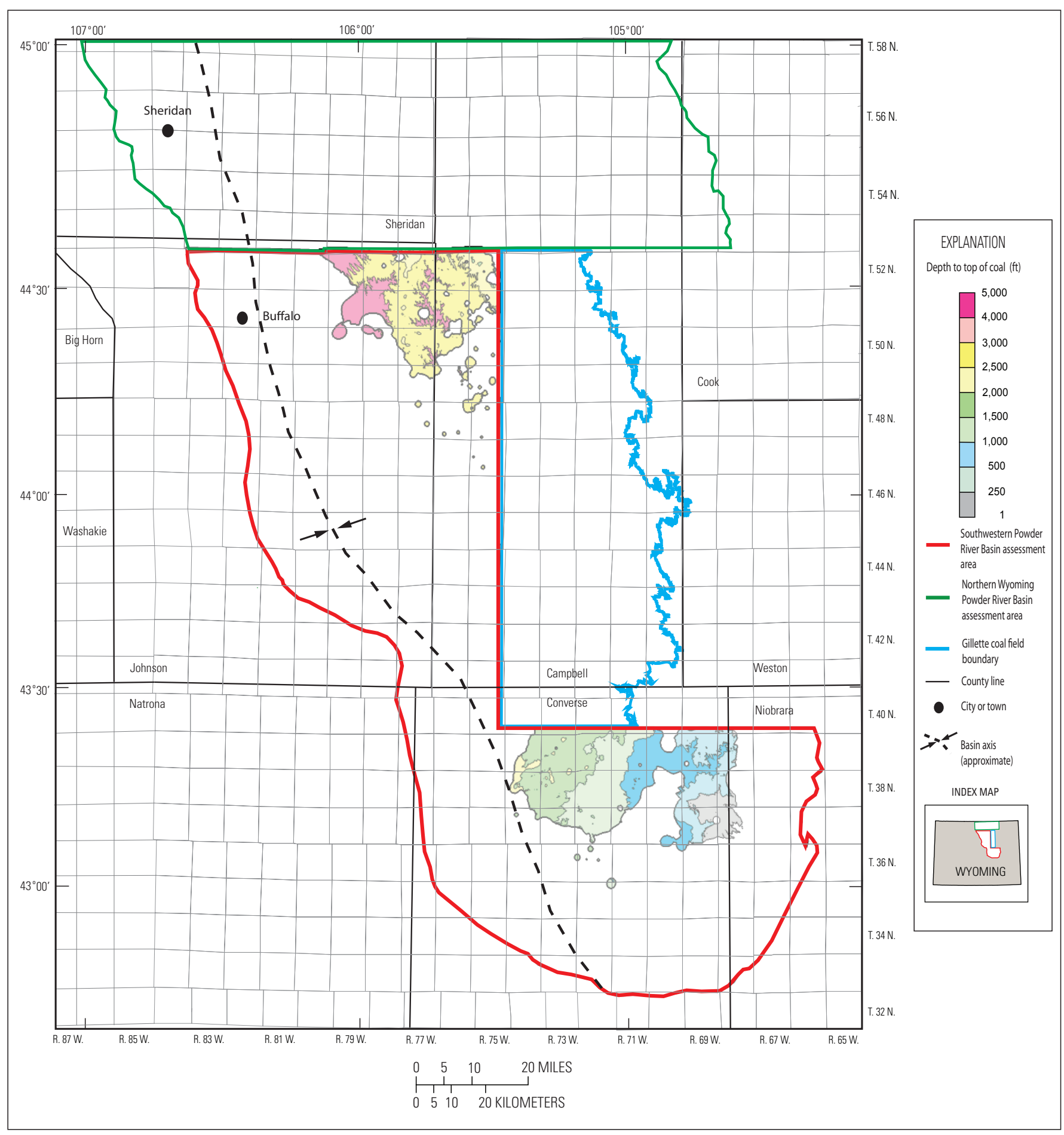

Figure 88. Depth to the top of the Roberts coal bed within the Southwestern Powder River Basin assessment area. 


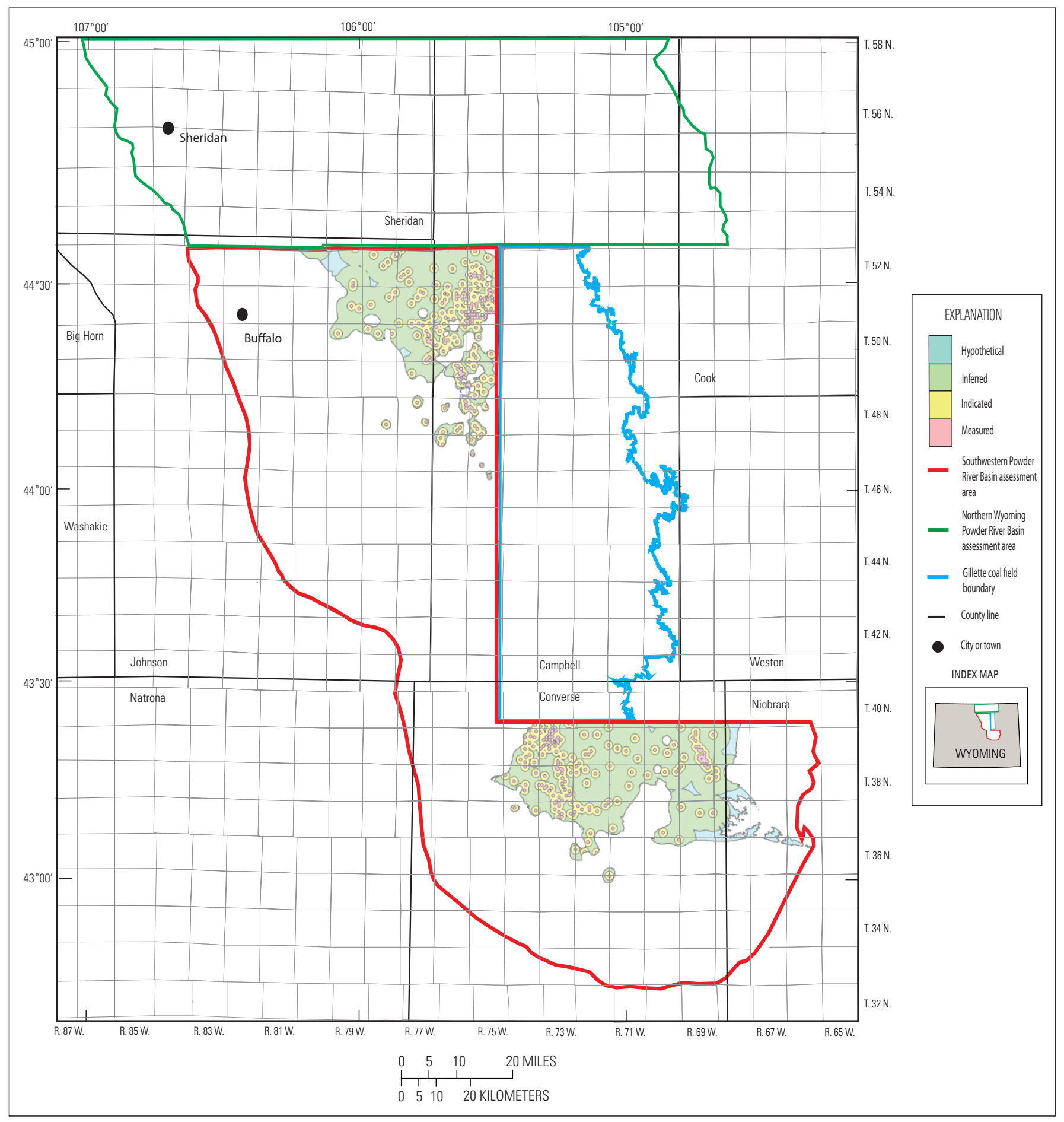

Figure 89. Coal resource reliability categories for the Roberts coal bed within the Southwestern Powder River Basin assessment area. 


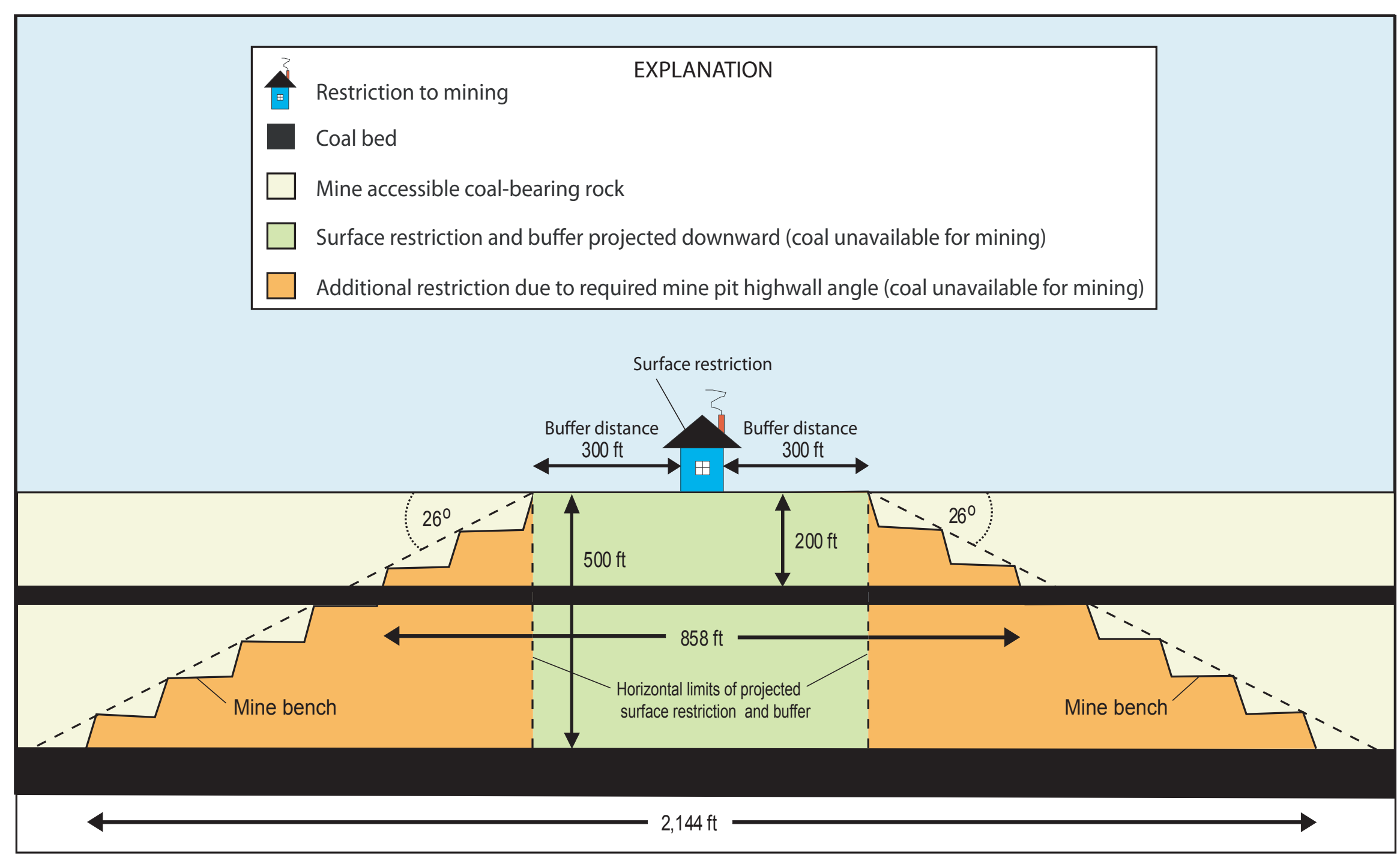

Figure 90. Illustration showing the effect of coal bed depth upon restricted resource due to mine pit highwall setback requirements (not to scale). 


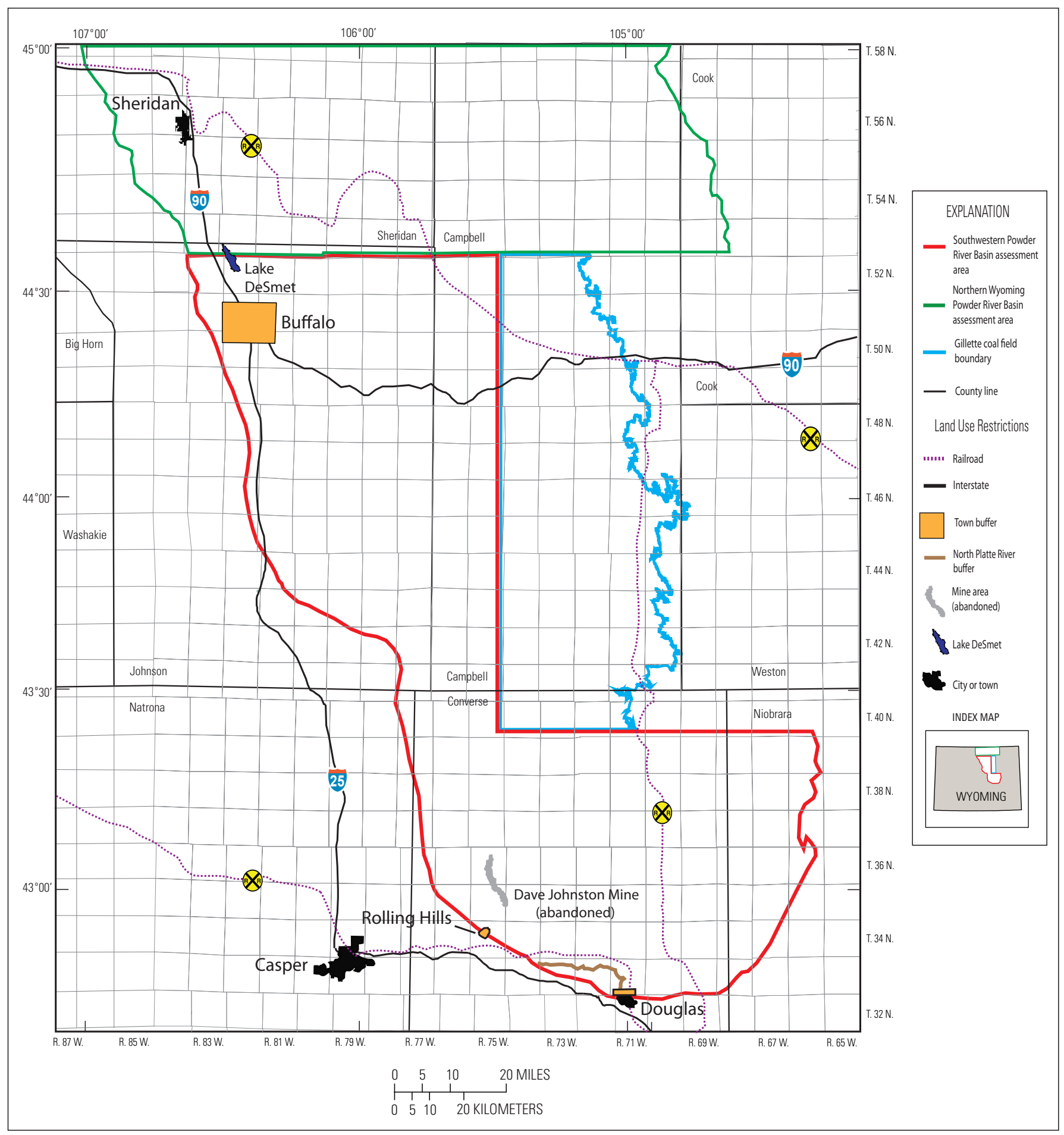

Figure 91. Land use restrictions in the Southwestern Powder River Basin assessment area. 


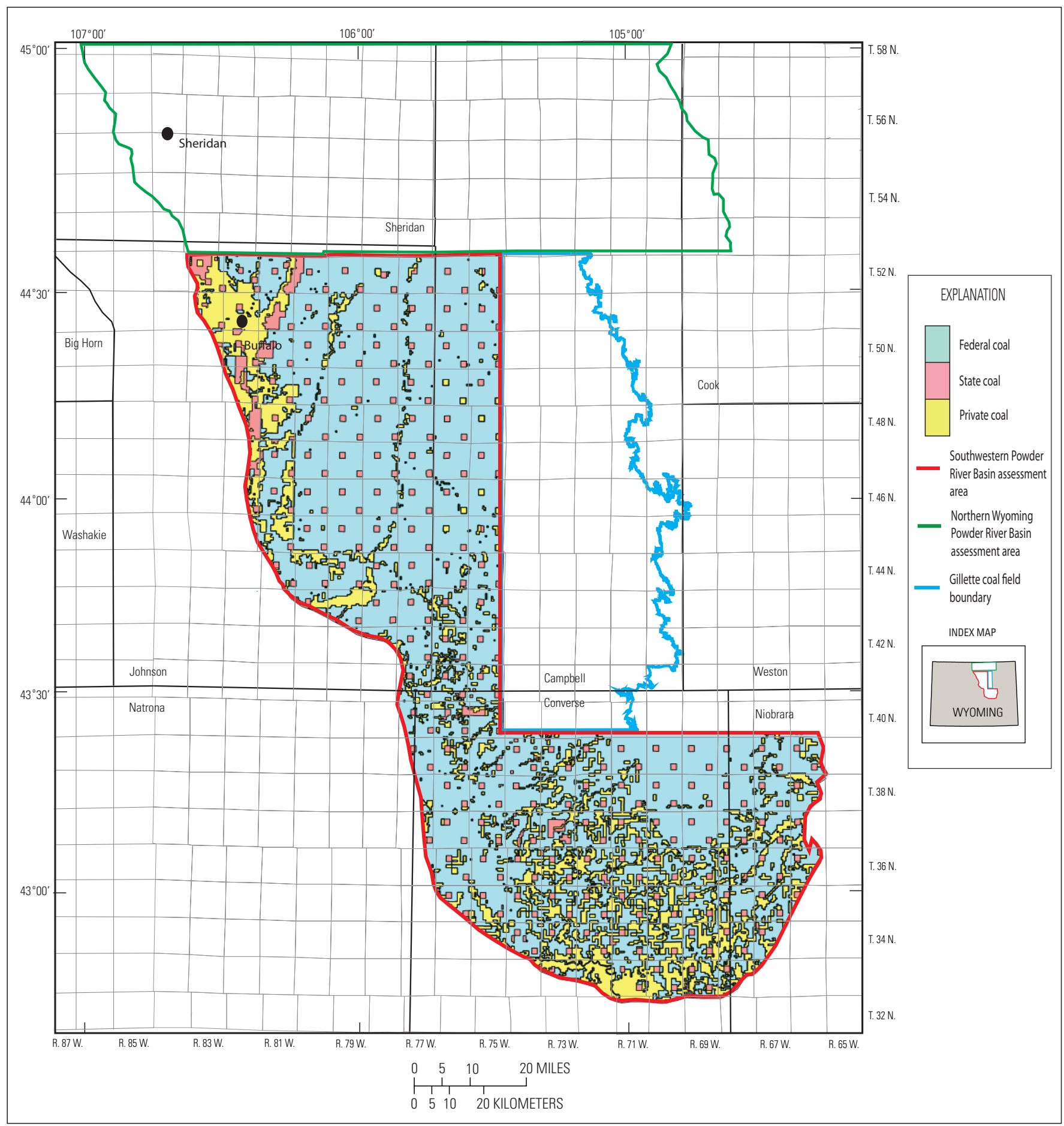

Figure 92. Coal ownership in the Southwestern Powder River Basin assessment area. 


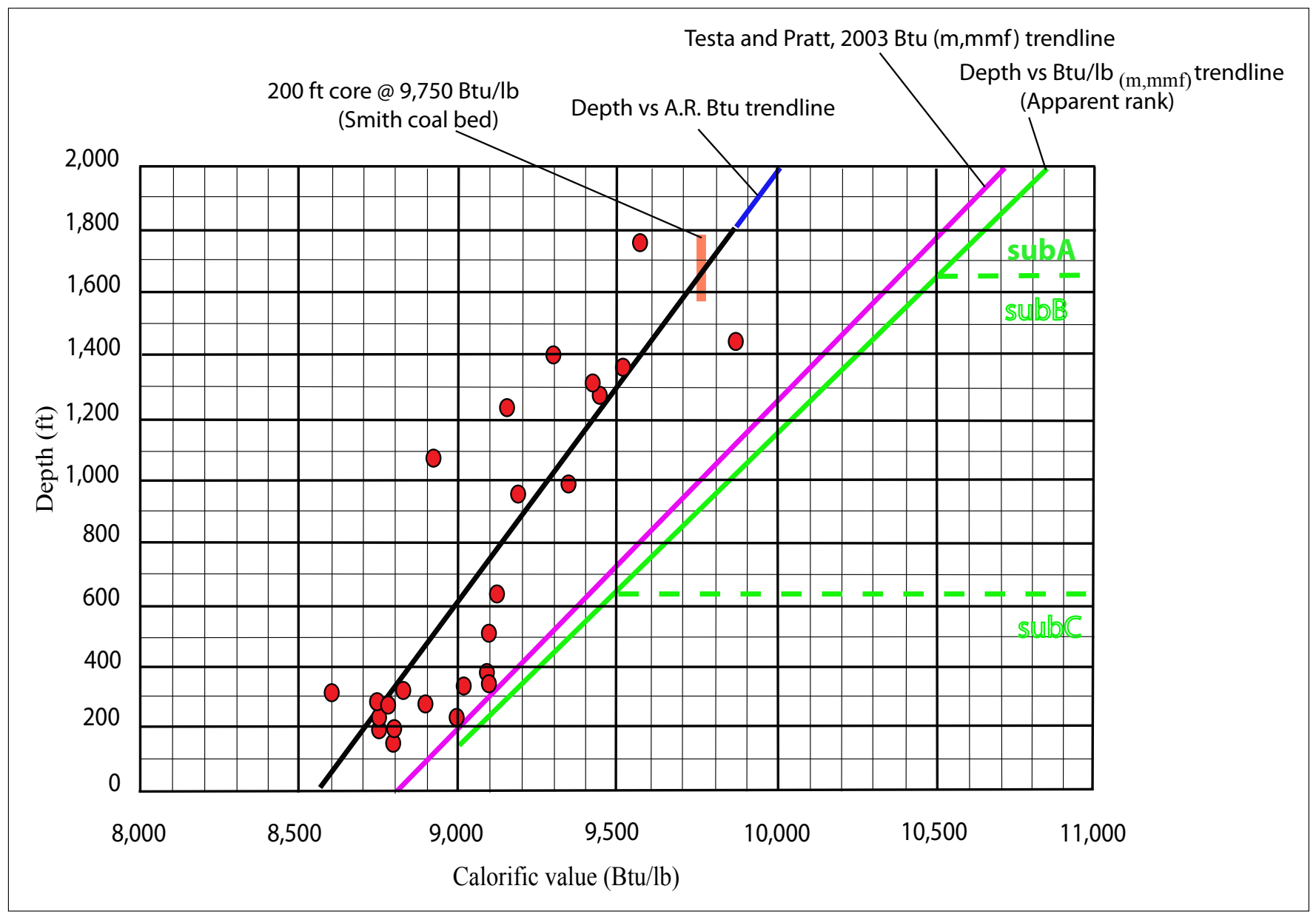

Figure 93. Estimated variability of as-received (AR) and moist, mineral-matter-free ( $\mathrm{m}$, mmf) heating content values versus depth for the Wyodak-Anderson coal beds (fig. 6) in the Southwestern Powder River Basin assessment area. The $\mathrm{m}, \mathrm{mmf}$ heating content values are used to determine apparent rank and the ranges are (1) subbituminous $C$ (sub C) $<9,500 \mathrm{Btu} / \mathrm{lb}_{\mathrm{m}, \mathrm{mm} f^{\prime}}$ (2) subbituminous $\mathrm{B}$ (sub B) 9,500-10,500 Btu/lb $\mathrm{b}_{\mathrm{m}, \mathrm{mmf}}$; and (3) subbituminous $A$ (sub A) >10,500 Btu/lb ${ }_{m, m m f}$ ('ASTM, 2008). 


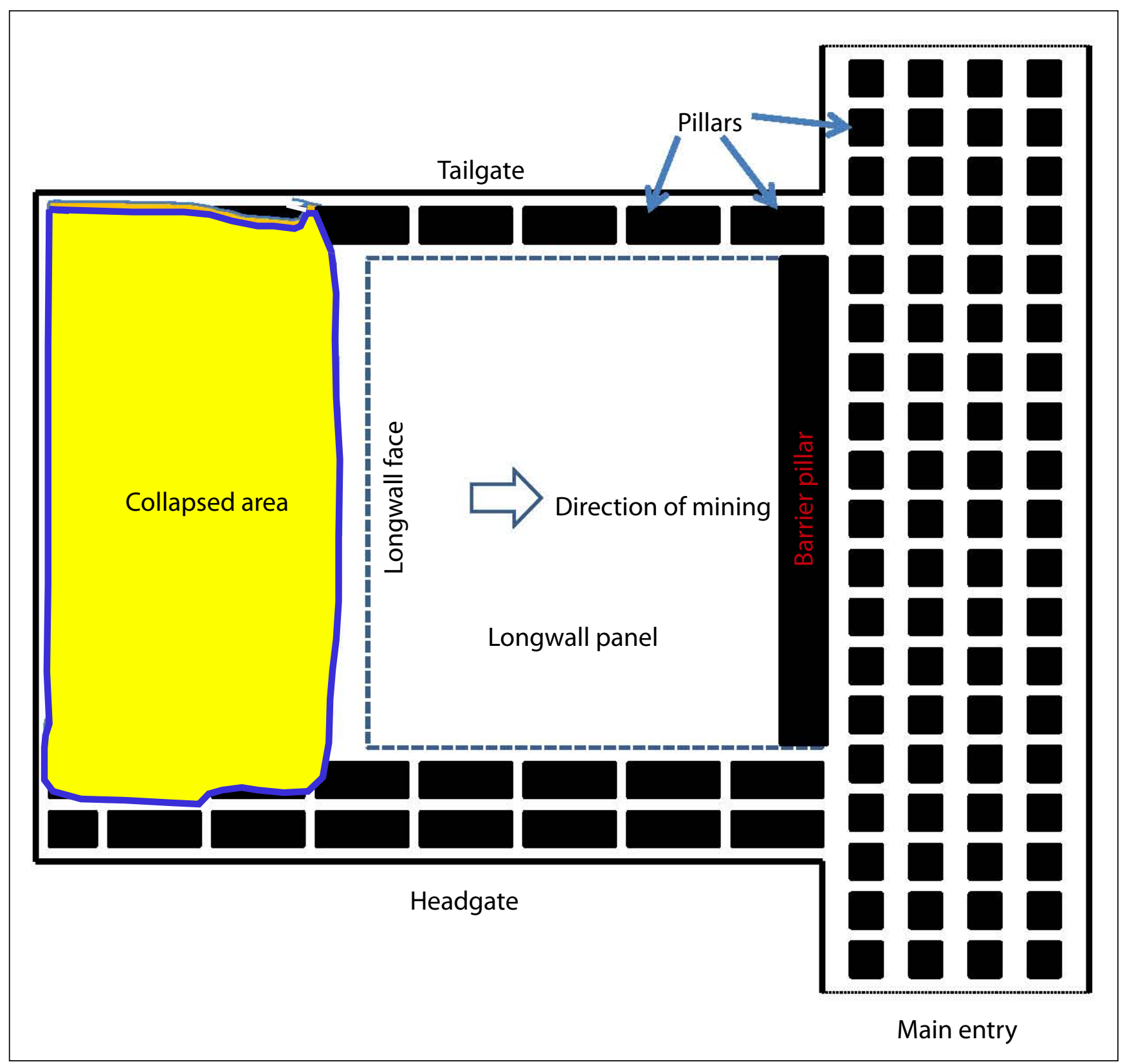

Figure 94. Diagram of typical longwall mining operation. 


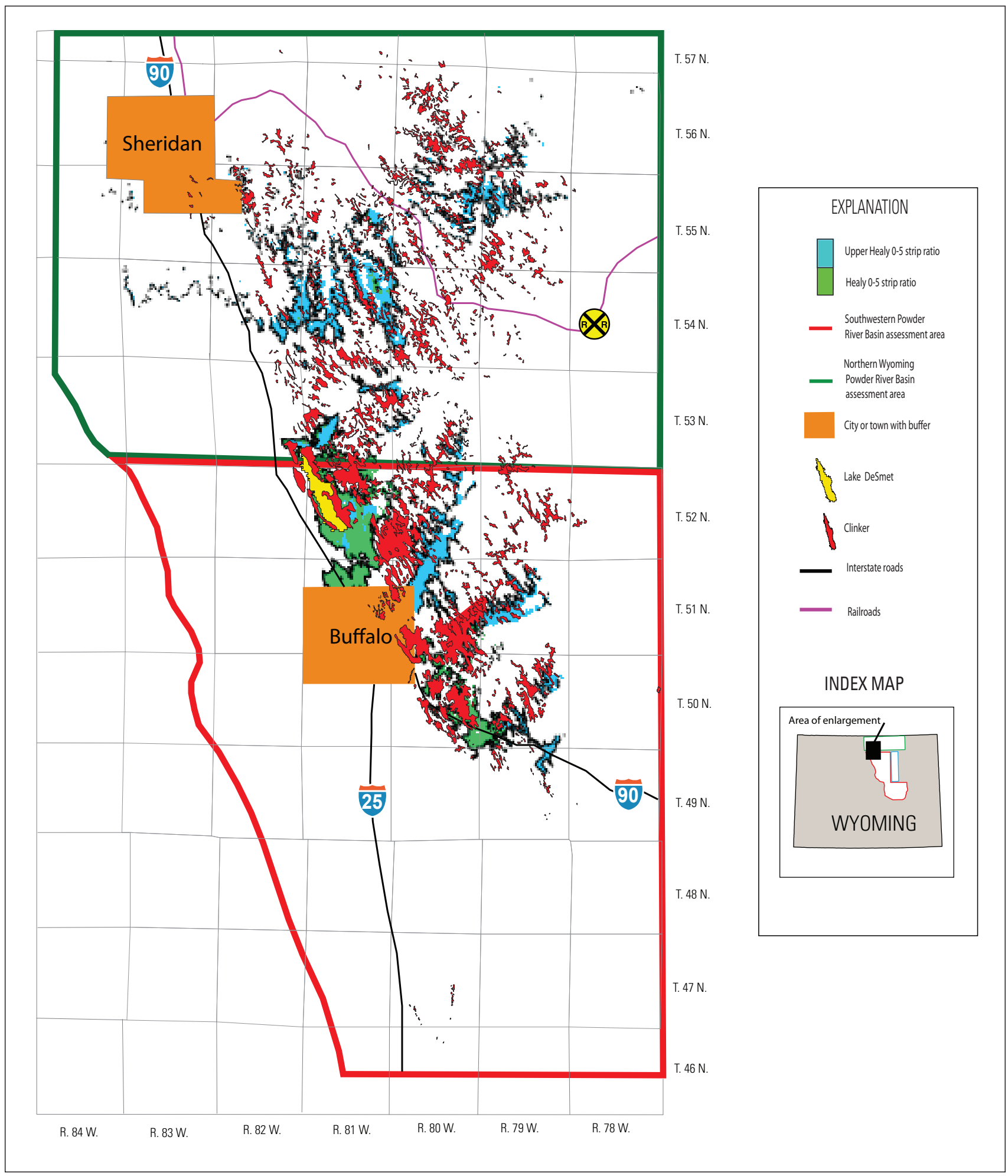

Figure 95. Areal extent for the Upper Healy and Healy coal beds where the stripping ratios are up to 5:1, thickness are equal to at least $5.0 \mathrm{ft}$, and the overburden is less than or equal to $500 \mathrm{ft}$ thick. 


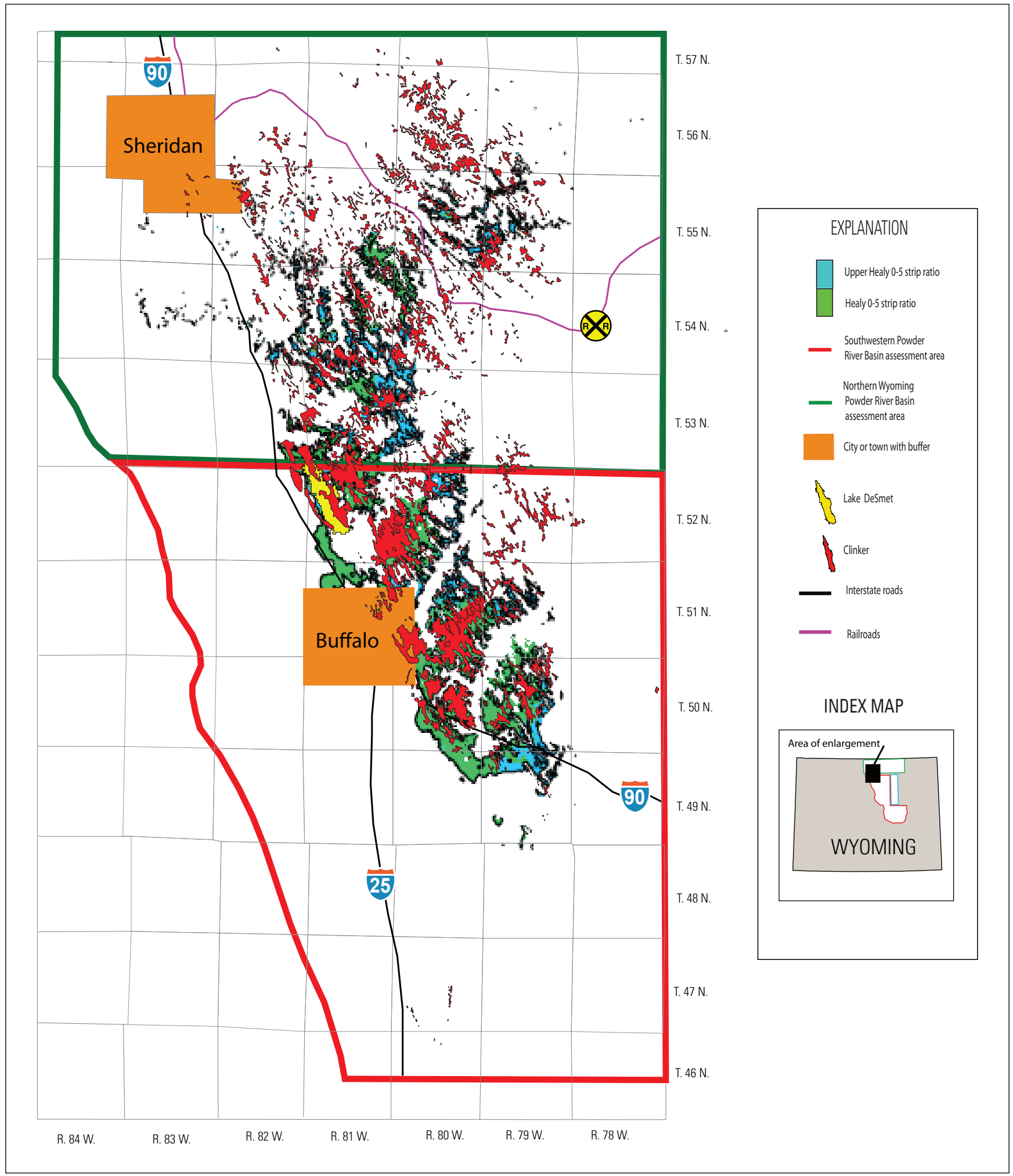

Figure 96. Areal extent for the Upper Healy and Healy coal beds where the stripping ratios are from 5:1 to 5:10, thickness are equal to at least $5.0 \mathrm{ft}$, and the overburden is less than or equal to $500 \mathrm{ft}$ thick. 